\title{
Chiral Ugi-Type Amines: Practical Synthesis, Ligand Development, and Asymmetric Catalysis
}

\author{
Wu-Wei Dong, ${ }^{\dagger}$ Yi-Nan Li, ${ }^{\dagger}$ Xin Chang,${ }^{\dagger}$ Chong Shen, ${ }^{\dagger}$ Chun-Jiang Wang $* \dagger, \ldots$ \\ $\dagger$ Engineering Research Center of Organosilicon Compounds \& Materials, Ministry of Education, College of \\ Chemistry and Molecular Sciences, Wuhan University, Wuhan 430072, China $₫$ State Key Laboratory of \\ Elemento-organic Chemistry, Nankai University, Tianjin 300071, China \\ E-mail: cjwang@whu.edu.cn
}

Table of Contents

1. General Remarks . $\mathrm{S} 2$

2. Synthesis of Ferrocenecarboxaldehyde Derived imines $\mathbf{8}$ and $\mathbf{1 1}$ .S2-S4

3. Initial Study on the Synthesis of Chiral Ugi-Type Amine Derivative. .S4-S6

4. Experimental Results of the Reaction of Aldimine $\mathbf{1 1}$ with Other Allylic Carbonates. S6-S9

5. Practical Preparation of Enantioenriched Ugi-Type Amine Derivative $(S)-\mathbf{1 5}$ and $(S)-\mathbf{1 6} \ldots . . S 9-S 12$

6. Synthesis of Chiral PPFA-Type and Josiphos-Type Ligands S13-S17

7. $\mathrm{Cu}(\mathrm{I}) /\left(S, R_{p}\right)$-17b-Catalyzed 1,3-Dipolar Cycloaddition Reactions of Azomethine Ylides..S17-S20

8. Optimization of $\mathrm{Cu}(\mathrm{I})$-Catalyzed Asymmetric Michael Addition of Grignard Reagent 24a to $\alpha, \beta$-Unsaturated Ester 23a. . $\mathrm{S} 21$

9. Scope of $\mathrm{Cu}(\mathrm{I}) /\left(S, R_{p}\right)$-L3-Catalyzed Asymmetric Michael Addition of Grignard Reagents to $\alpha, \beta$-Unsaturated Esters. S22-S29

10. References. S30

11. NMR, HPLC and GC Spectra. $. S 31-S 128$ 


\section{General Remarks}

Unless otherwise stated, all reactions were set up under nitrogen atmosphere in oven-dried glassware using standard Schlenk techniques, monitored by TLC with silica-gel coated plates and purified by flash column chromatography. Commercially available reagents were used without further purification. Solvents were purified prior to use according to the standard methods. $(S, S, S)-\mathbf{L} 2^{1}$ and $\mathbf{L 3}-\mathbf{L} 6^{2}$ were prepared according to the literature procedure. ${ }^{1} \mathrm{H}$ NMR spectra were recorded on a Bruker $400 \mathrm{MHz}$ spectrometer in $\mathrm{CDCl}_{3}$, chemical shifts are reported in ppm with the internal TMS signal at $0.0 \mathrm{ppm}$ as a standard. The data are reported as $(\mathrm{s}=$ single, $\mathrm{d}=$ double, $\mathrm{t}=$ triple, $\mathrm{q}=$ quarte, $\mathrm{m}=$ multiple or unresolved, brs = broad single, coupling constant $(\mathrm{s})$ in $\mathrm{Hz}$, integration). ${ }^{13} \mathrm{C}$ NMR spectra were recorded on a Bruker $101 \mathrm{MHz}$ spectrometer in $\mathrm{CDCl}_{3}$, chemical shifts are reported in ppm with the internal chloroform signal at $77.0 \mathrm{ppm}$ as a standard. ${ }^{19} \mathrm{~F}$ NMR spectra were recorded on a Bruker $376 \mathrm{MHz}$ spectrometer in $\mathrm{CDCl}_{3}$, chemical shifts are reported in ppm with the internal $\mathrm{CF}_{3} \mathrm{COOH}$ signal at -76.55 ppm. ${ }^{31} \mathrm{P}$ NMR spectra were recorded on a Bruker $162 \mathrm{MHz}$ spectrometer in $\mathrm{CDCl}_{3}$, chemical shifts are reported in ppm with the external $85 \% \mathrm{H}_{3} \mathrm{PO}_{4}$ signal at $0.0 \mathrm{ppm}$ as a standard. The enantiomeric excesses $(e e)$ of the products were determined by chiral-phase HPLC and GC analysis. Optical rotations were measured on an Rudolph Research Analytical Autopol VI polarimeter with $[\alpha]_{D}$ values reported in degrees; concentration (c) is in g/100 $\mathrm{mL}$. The absolute configuration of compound $(\boldsymbol{S})-\mathbf{1 6} \cdot \mathrm{BH}_{3}$ was determined unequivocally according to the X-ray diffraction analysis.

\section{Synthesis of Ferrocenecarboxaldehyde Derived imines 8 and $\mathbf{1 1}^{3,4}$}

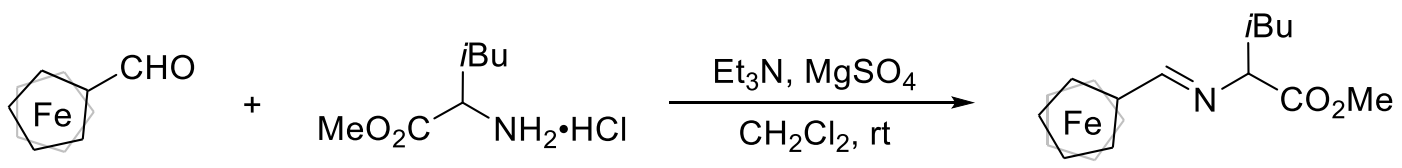

To a suspension of methyl $l$-leucinate hydrochloride (12 mmol) and $\mathrm{MgSO}_{4}$ in $\mathrm{CH}_{2} \mathrm{Cl}_{2}(20 \mathrm{~mL})$ was added $\mathrm{Et}_{3} \mathrm{~N}(10.2 \mathrm{~mL}, 14 \mathrm{mmol})$. The mixture was stirred at room temperature for $1 \mathrm{~h}$, and then ferrocenecarboxaldehyde $(10 \mathrm{mmol})$ was added. The reaction was stirred at room temperature 
overnight, and then the resulting precipitate was removed by filtration. The filtrate was washed with water, the aqueous phase was extracted with $\mathrm{CH}_{2} \mathrm{Cl}_{2}$ and the combined organic phase was washed with water five times, dried over $\mathrm{MgSO}_{4}$ and concentrated. The resulting aldimine $\mathbf{8}$ were used directly without further purification.<smiles>CC(=O)C(CC(C)C)N=Cc1ccccc1</smiles>

\section{methyl $(E)-2$-(ferrocenylideneamino)-4-methylpentanoate (8)}

Yield (99\%); orange solid; m.p. $=68-70{ }^{\circ} \mathrm{C} ; \quad{ }^{1} \mathrm{H}$ NMR $\left(400 \mathrm{MHz}, \mathrm{CDCl}_{3}\right) \delta 8.16(\mathrm{~s}, 1 \mathrm{H}), 4.71-$ $4.68(\mathrm{~m}, 2 \mathrm{H}), 4.40-4.38(\mathrm{~m}, J=3.2,2 \mathrm{H}), 4.19(\mathrm{~s}, 5 \mathrm{H}), 3.96-3.92(\mathrm{~m}, 1 \mathrm{H}), 3.73(\mathrm{~s}, 3 \mathrm{H}), 1.91-$ $1.83(\mathrm{~m}, 1 \mathrm{H}), 1.78-1.71(\mathrm{~m}, 1 \mathrm{H}), 1.66-1.54(\mathrm{~m}, 1 \mathrm{H}), 0.97(\mathrm{dd}, J=6.7,1.1 \mathrm{~Hz}, 3 \mathrm{H}), 0.91(\mathrm{dd}, J=$ 6.6, 1.1 Hz, 3H). ${ }^{13} \mathrm{C}$ NMR (101 MHz, $\left.\mathrm{CDCl}_{3}\right) \delta 173.0,163.6,79.7,71.7,70.8,70.7,69.1,68.9,68.8$, 52.1, 41.7, 24.3, 23.3, 21.2. HRMS(ESI+) Calcd. For $\mathrm{C}_{18} \mathrm{H}_{24} \mathrm{FeNO}_{2}\left([\mathrm{M}+\mathrm{H}]^{+}\right)$: 342.1151, found: 342.1141 .
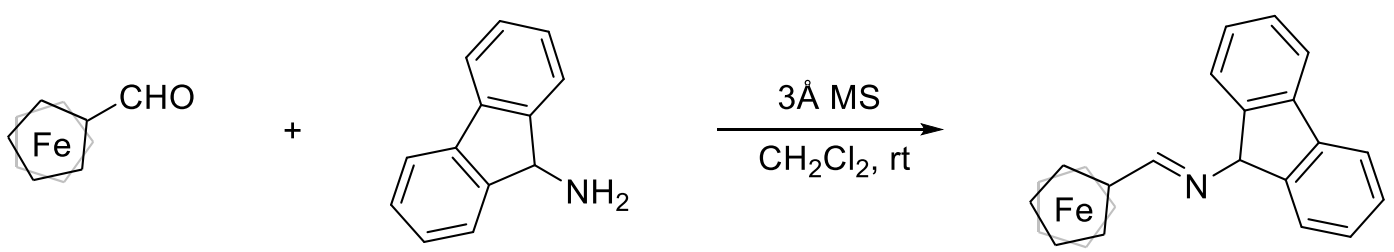

To a suspension of 9-aminofluorene $(100 \mathrm{mmol})$ and $3 \AA \mathrm{MS}(100 \mathrm{~g})$ in $\mathrm{CH}_{2} \mathrm{Cl}_{2}(200 \mathrm{~mL})$ was added a $\mathrm{CH}_{2} \mathrm{Cl}_{2}$ solution of ferrocenecarboxaldehyde $(100 \mathrm{mmol})$ via syringe. The reaction mixture was stirred at room temperature until complete consumption of starting material. The reaction was diluted with $200 \mathrm{~mL} \mathrm{CH}_{2} \mathrm{Cl}_{2}$, and then the resulting precipitate was removed by filtration. The residue was washed with $200 \mathrm{~mL} \mathrm{CH}_{2} \mathrm{Cl}_{2}$ five times, and the combined organic phase was washed with brine, dried over $\mathrm{MgSO}_{4}$ and concentrated. The resulting aldimine $\mathbf{1 1}$ were used directly without further purification. 


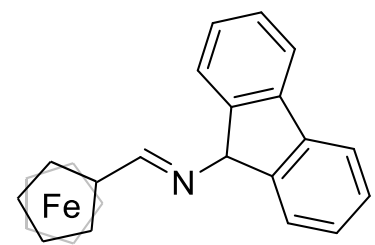

\section{(E)-N-(9H-fluoren-9-yl)-1-ferrocenylmethanimine (11)}

Yield (>99\%); orange solid; m.p. $=196-198{ }^{\circ} \mathrm{C}{ }^{1} \mathrm{H}$ NMR $\left(400 \mathrm{MHz}, \mathrm{CDCl}_{3}\right) \delta 8.60(\mathrm{~s}, 1 \mathrm{H}), 7.75(\mathrm{~d}$, $J=8.2 \mathrm{~Hz}, 2 \mathrm{H}), 7.44-7.37(\mathrm{~m}, 4 \mathrm{H}), 7.34-7.28(\mathrm{~m}, 2 \mathrm{H}), 5.29(\mathrm{~s}, 1 \mathrm{H}), 4.75-4.67(\mathrm{~m}, 2 \mathrm{H}), 4.40(\mathrm{t}$, $J=2.0 \mathrm{~Hz}, 2 \mathrm{H}), 4.22(\mathrm{~s}, 5 \mathrm{H}) ;{ }^{13} \mathrm{C} \mathrm{NMR}\left(101 \mathrm{MHz}, \mathrm{CDCl}_{3}\right) \delta 163.9,145.0,141.0,128.4,127.5$, 125.1, 120.1, 80.3, 74.9, 70.8, 69.3, 68.9; HRMS(ESI+) Calcd. For $\mathrm{C}_{24} \mathrm{H}_{20} \mathrm{FeN}\left([\mathrm{M}+\mathrm{H}]^{+}\right)$: 378.0940, found: 378.0934.

\section{Initial Study on the Synthesis of Chiral Ugi-Type Amine Derivative ${ }^{4,5}$}

\section{Method A:}

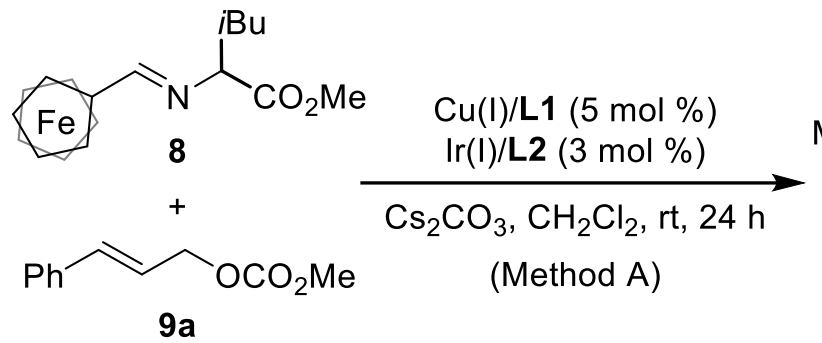

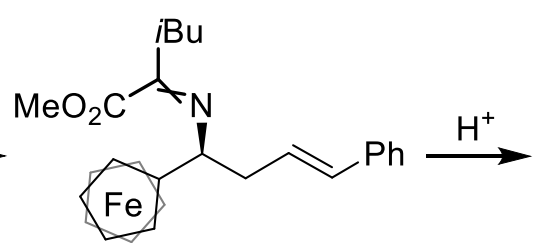

10

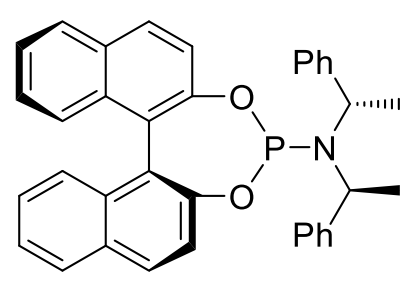

$(S, S, S)$-L2<smiles>NC(C/C=C/Pc1ccccc1)c1ccccc1</smiles>

A flame dried Schlenk tube I was cooled to room temperature and filled with $\mathrm{N}_{2}$. To this flask were added $\left[\operatorname{Ir}(\mathrm{COD}) \mathrm{Cl}_{2}(1.5 \mathrm{~mol} \%)\right.$, phosphoramidite ligand $(S, S, S)-\mathbf{L} 2(3 \mathrm{~mol} \%), 1 \mathrm{~mL}$ degassed THF, degassed $n$-propylamine $(0.5 \mathrm{~mL})$. The reaction mixture was heated at $50{ }^{\circ} \mathrm{C}$ for $30 \mathrm{~min}$ and then the volatile solvent was removed under vacuum to give the iridium complex as a pale yellow solid. Meanwhile, in a separated Schlenk tube II, DPEPhos $(5.5 \mathrm{~mol} \%)$ and $\mathrm{Cu}(\mathrm{MeCN})_{4} \mathrm{BF}_{4}(5$ mol \%) were dissolved in DCM $(0.5 \mathrm{~mL})$, and stirred at room temperature for $0.5 \mathrm{~h}$. The 
$\mathrm{Cu} / \mathrm{DPEphos}$ complex solution was then transferred to the tube I containing iridium complex via syringe. Cinnamyl carbonate 9a $(0.5 \mathrm{mmol})$, leucine derived aldimine ester $\mathbf{8}(0.6 \mathrm{mmol})$ and $\mathrm{Cs}_{2} \mathrm{CO}_{3}$ $(0.75 \mathrm{mmol})$ in DCM $(1.5 \mathrm{~mL})$ were then added. The cascade reaction was finished smoothly in $24 \mathrm{~h}$ (monitored by ${ }^{1} \mathrm{H}$ NMR spectroscopy). The organic solvent was removed by rotary evaporation and the residue was purified by column chromatography to give the oil compound $\mathbf{1 0}$ as E/Z mixture (95\% yield). The enantiomeric excess of the compound $\mathbf{1 0}$ was determined to be $97 \%$ ee by HPLC analysis of the derived primary amine 15a, which was obtained through acidic hydrolysis.

\section{Method B:}

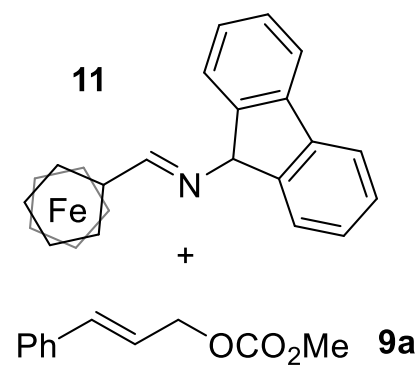

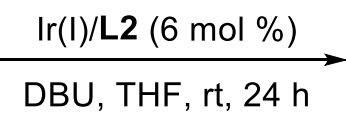

(Method B)

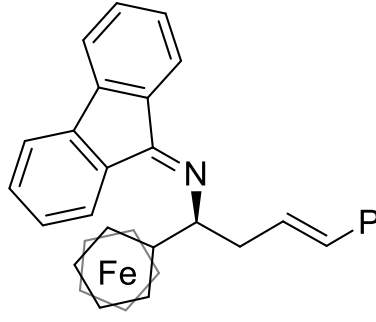

$12 a$

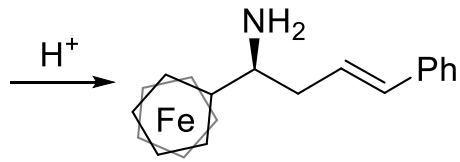

$15 a$

A flame dried Schlenk tube was cooled to room temperature and filled with $\mathrm{N}_{2}$. To this flask were added $[\operatorname{Ir}(\mathrm{COD}) \mathrm{Cl}]_{2}(3 \mathrm{~mol} \%)$, phosphoramidite ligand $(S, S, S a)-\mathbf{L} 2(6 \mathrm{~mol} \%), 1 \mathrm{~mL}$ degassed THF, degassed $n$-propylamine $(0.5 \mathrm{~mL})$. The reaction mixture was heated at $50{ }^{\circ} \mathrm{C}$ for 30 min and then the volatile solvent was removed under vacuum to give the iridium complex as a pale yellow solid. The Schlenk tube was refilled with $\mathrm{N}_{2}$. To this flask were added aldimine 11 (0.5 mmol) in $0.5 \mathrm{~mL}$ of THF. Then, cinnamyl carbonate $9 \mathrm{a}(0.55 \mathrm{mmol})$ and DBU $(0.5 \mathrm{mmol})$ in THF $(1.5 \mathrm{~mL})$ were then added. The reaction was then stirred at room temperature until complete consumption of starting material (detected by TLC). The organic solvent was removed by rotary evaporation and the residue was purified by column chromatography to give the solid compound 12a (96\% yield). The enantiomeric excess of the compound 12a was determined to be $96 \%$ ee by HPLC analysis of the derived primary amine $\mathbf{1 5 a}$, which was obtained through acidic hydrolysis. 
<smiles>NC(C/C=C/c1ccccc1)c1ccccc1</smiles>

\section{(S, E)-1-ferrocenyl-4-phenylbut-3-en-1-amine (15a)}

Yield (98\%); yellow solid; m.p. $=54-56{ }^{\circ} \mathrm{C} ;[\alpha]^{28} \mathrm{D}=+19.1\left(c 0.11, \mathrm{CHCl}_{3}\right) ;{ }^{1} \mathrm{H}$ NMR $(400 \mathrm{MHz}$, $\left.\mathrm{CDCl}_{3}\right) \delta 7.36-7.28(\mathrm{~m}, 4 \mathrm{H}), 7.23-7.19(\mathrm{~m}, 1 \mathrm{H}), 6.46(\mathrm{~d}, J=15.8 \mathrm{~Hz}, 1 \mathrm{H}), 6.20(\mathrm{dt}, J=15.8,7.3$ $\mathrm{Hz}, 1 \mathrm{H}), 4.24-4.23(\mathrm{~m}, 1 \mathrm{H}), 4.17-4.13(\mathrm{~m}, 8 \mathrm{H}), 3.83-3.79(\mathrm{~m}, 1 \mathrm{H}), 2.62-2.56(\mathrm{~m}, 1 \mathrm{H}), 2.50$ (bs, 2H), $2.42-2.35(\mathrm{~m}, 1 \mathrm{H}) .{ }^{13} \mathrm{C}$ NMR $\left(101 \mathrm{MHz}, \mathrm{CDCl}_{3}\right) \delta 137.4,132.7,128.6,127.2,127.1,126.1$, 94.1, 77.3, 68.4, 67.7, 67.5, 66.7, 65.5, 50.6, 42.8. HRMS(ESI+) Calcd. For: $\mathrm{C}_{20} \mathrm{H}_{21} \mathrm{FeN}\left([\mathrm{M}]^{+}\right)$: 331.1023, found: 331.1013. The product was analyzed by HPLC to determine the enantiomeric excess: $99 \%$ ee (Chiralpak OD-H, $i$-propanol $/$ hexane $=10 / 90$, flow rate $1.0 \mathrm{~mL} / \mathrm{min}, \lambda=254 \mathrm{~nm}$ ); retention times: $\mathrm{t}_{1}=6.95 \min$ (major), $\mathrm{t}_{2}=8.06 \min$ (minor).

\section{Experimental Results of the Reaction of Aldimine 11 with Other Allylic Carbonates ${ }^{4}$}

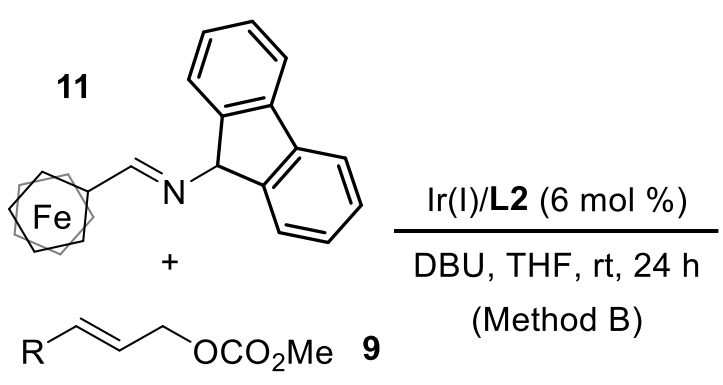

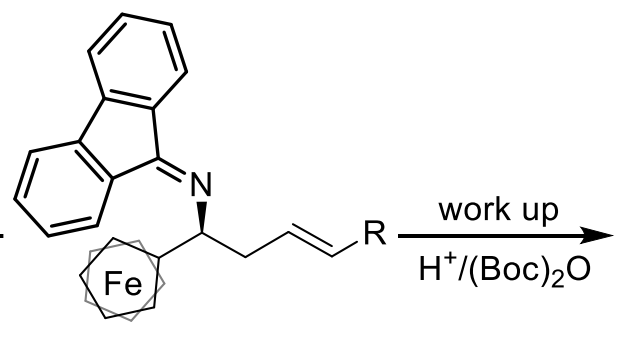

12

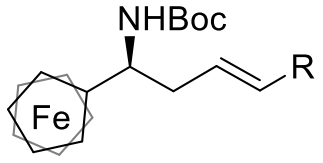

$15^{\prime}$

A flame dried Schlenk tube was cooled to room temperature and filled with $\mathrm{N}_{2}$. To this flask were added $[\operatorname{Ir}(\mathrm{COD}) \mathrm{Cl}]_{2}(3 \mathrm{~mol} \%)$, phosphoramidite ligand $(S, S, S a)-\mathbf{L} 2(6 \mathrm{~mol} \%), 1 \mathrm{~mL}$ degassed THF, degassed $n$-propylamine $(0.5 \mathrm{~mL})$. The reaction mixture was heated at $50{ }^{\circ} \mathrm{C}$ for 30 min and then the volatile solvent was removed under vacuum to give the iridium complex as a pale yellow solid. The Schlenk tube was refilled with $\mathrm{N}_{2}$. To this flask were added aldimine $\mathbf{1 1}(0.5 \mathrm{mmol})$ in $0.5 \mathrm{~mL}$ of THF. Then, allylic carbonate $9(0.55 \mathrm{mmol})$ and DBU $(0.5 \mathrm{mmol})$ in THF $(1.5 \mathrm{~mL})$ were then added. The reaction was then stirred at room temperature until complete consumption of starting material (detected by TLC). Then the solution was diluted with water and separated with separatory funnel. The aqueous phase was washed with DCM. Next, the aqueous phase was basified 
with $\mathrm{Et}_{3} \mathrm{~N}$ or $\mathrm{Na}_{2} \mathrm{CO}_{3}$, and then extracted with DCM. To the combined organic phase were added $2 \mathrm{~N}$ $\mathrm{NaOH}(5 \mathrm{~mL})$ and $\mathrm{Boc}_{2} \mathrm{O}(1.0 \mathrm{mmol})$, and then added and stirring at $\mathrm{rt}$ for $3 \mathrm{~h}$. The layers were separated, and the aqueous layer was extracted with DCM. The combined organic components were washed with saturated brine, dried over anhydrous $\mathrm{Na}_{2} \mathrm{SO}_{4}$, filtration and evaporated in vacuum. The residue was purified by column chromatography to give the desired product, which was then directly analyzed by HPLC to determine the enantiomeric excess.

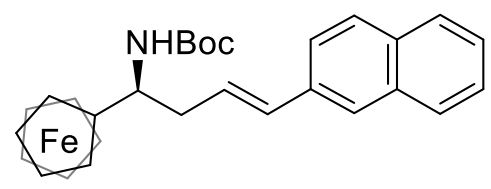

\section{tert-butyl $(S, E)$-(1-ferrocenyl-4-(naphthalen-2-yl)but-3-en-1-yl)carbamate (15b')}

Yield $(220 \mathrm{mg}, 92 \%)$; yellow solid; m.p. $=130-132{ }^{\circ} \mathrm{C} ;[\alpha]^{28} \mathrm{D}=-5.5\left(c 0.11, \mathrm{CHCl}_{3}\right) ;{ }^{1} \mathrm{H}$ NMR $\left(400 \mathrm{MHz}, \mathrm{CDCl}_{3}\right) \delta 7.76-7.72(\mathrm{~m}, 3 \mathrm{H}), 7.65(\mathrm{~d}, J=1.7 \mathrm{~Hz}, 1 \mathrm{H}), 7.54(\mathrm{dd}, J=8.6,1.8 \mathrm{~Hz}, 1 \mathrm{H})$, $7.44-7.37(\mathrm{~m}, 2 \mathrm{H}), 6.57(\mathrm{~d}, J=15.8 \mathrm{~Hz}, 1 \mathrm{H}), 6.31(\mathrm{dt}, J=15.2,7.2 \mathrm{~Hz}, 1 \mathrm{H}), 4.73-4.67(\mathrm{~m}, 1 \mathrm{H})$, $4.17-4.11(\mathrm{~m}, 9 \mathrm{H}), 2.76-2.69(\mathrm{~m}, 1 \mathrm{H}), 2.59-2.51(\mathrm{~m}, 1 \mathrm{H}), 1.44(\mathrm{~s}, 10 \mathrm{H}) .{ }^{13} \mathrm{C}$ NMR $(101 \mathrm{MHz}$, $\left.\mathrm{CDCl}_{3}\right) \delta 155.4,135.0,133.7,132.8,132.6,128.1,127.9,127.7,127.0,126.2,125.7,125.7,123.7$, 91.0, 79.4, 68.7, 67.8, 67.7, 66.9, 66.2, 40.8, 28.5. HRMS(ESI+) Calcd. For: $\mathrm{C}_{29} \mathrm{H}_{31} \mathrm{FeNO}_{2}\left([\mathrm{M}]^{+}\right)$: 481.1699, found: 481.1693. The product was analyzed by HPLC to determine the enantiomeric excess: $97 \%$ ee (Chiralpak AD-H, $i$-propanol/hexane $=10 / 90$, flow rate $1.0 \mathrm{~mL} / \mathrm{min}, \lambda=254 \mathrm{~nm}$ ); retention times: $\mathrm{t}_{1}=11.73 \min$ (major), $\mathrm{t}_{2}=29.66 \mathrm{~min}$ (minor).

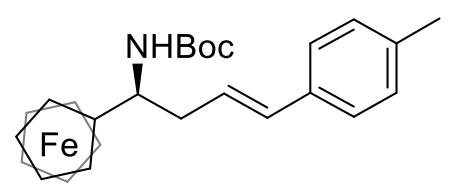

\section{tert-butyl $(S, E)$-(1-ferrocenyl-4-(p-tolyl)but-3-en-1-yl)carbamate (15c')}

Yield (196 mg, 89\%); yellow solid; M.p. $=110-112{ }^{\circ} \mathrm{C} ;[\alpha]^{28} \mathrm{D}=+15.0\left(c 0.12, \mathrm{CHCl}_{3}\right) ;{ }^{1} \mathrm{H}$ NMR $\left(400 \mathrm{MHz}, \mathrm{CDCl}_{3}\right) \delta 7.22(\mathrm{~d}, J=7.9 \mathrm{~Hz}, 2 \mathrm{H}), 7.09(\mathrm{~d}, J=7.9 \mathrm{~Hz}, 2 \mathrm{H}), 6.39(\mathrm{~d}, J=15.7 \mathrm{~Hz}, 1 \mathrm{H})$, $6.16-6.08(\mathrm{~m}, 1 \mathrm{H}), 4.66(\mathrm{~m}, 1 \mathrm{H}), 4.18(\mathrm{~s}, 5 \mathrm{H}), 4.16-4.10(\mathrm{~m}, 4 \mathrm{H}), 2.70-2.64(\mathrm{~m}, 1 \mathrm{H}), 2.55-$ $2.47(\mathrm{~m}, 1 \mathrm{H}), 2.32$ (s, 3H), 1.45 (s, 9H). ${ }^{13} \mathrm{C} \mathrm{NMR}\left(101 \mathrm{MHz}, \mathrm{CDCl}_{3}\right) \delta 155.3,136.8,134.7,132.4$, $129.2,126.0,125.3,91.1,79.3,68.7,67.7,67.6,66.9,66.2,49.4,40.6,28.5,21.2$. HRMS(ESI+) 
Calcd. For: $\mathrm{C}_{26} \mathrm{H}_{31} \mathrm{FeNO}_{2}\left([\mathrm{M}]^{+}\right)$: 445.1699, found: 445.1693 . The product was analyzed by HPLC to determine the enantiomeric excess: $97 \%$ ee (Chiralpak AD-H, $i$-propanol/hexane $=10 / 90$, flow rate $1.0 \mathrm{~mL} / \mathrm{min}, \lambda=254 \mathrm{~nm}$ ); retention times: $\mathrm{t}_{1}=8.50 \mathrm{~min}$ (major), $\mathrm{t}_{2}=14.78 \mathrm{~min}$ (minor).

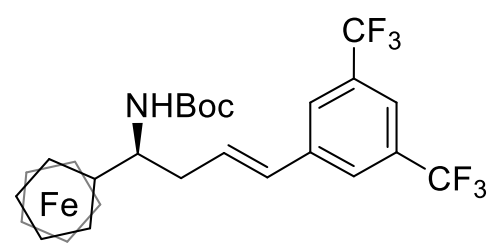

tert-butyl (S, E)-(4-(3,5-bis(trifluoromethyl)phenyl)-1-ferrocenylbut-3-en-1-yl)carbamate (15d') Yield (241 mg, 85\%); yellow oil; $[\alpha]^{28} \mathrm{D}=-14.3\left(c 0.11, \mathrm{CHCl}_{3}\right) ;{ }^{1} \mathrm{H} \mathrm{NMR}\left(400 \mathrm{MHz}, \mathrm{CDCl}_{3}\right) \delta 7.74$ $(\mathrm{d}, J=1.6 \mathrm{~Hz}, 2 \mathrm{H}), 7.69(\mathrm{~s}, 1 \mathrm{H}), 6.49(\mathrm{~d}, J=15.9 \mathrm{~Hz}, 1 \mathrm{H}), 6.39(\mathrm{dt}, J=15.9,6.9 \mathrm{~Hz}, 1 \mathrm{H}), 4.73-$ $4.60(\mathrm{~m}, 1 \mathrm{H}), 4.20(\mathrm{~s}, 5 \mathrm{H}), 4.18-4.14(\mathrm{~m}, 4 \mathrm{H}), 2.80-2.74(\mathrm{~m}, 1 \mathrm{H}), 2.58-2.58(\mathrm{~m}, 1 \mathrm{H}), 1.44(\mathrm{~s}$, 9H). ${ }^{13} \mathrm{C}$ NMR $\left(101 \mathrm{MHz}, \mathrm{CDCl}_{3}\right) \delta 155.3,139.6,131.8(\mathrm{q}, J=33.3 \mathrm{~Hz}) 131.4,129.6,125.9(\mathrm{~d}, \mathrm{~J}=$ $4.2 \mathrm{~Hz}), 123.4$ (q, 273.6 Hz), 120.4, 90.5, 79.6, 77.2, 68.7, 68.0, 67.7, 66.8, 66.0, 49.2, 40.8, 28.4. ${ }^{19} \mathrm{~F}$ NMR (376 MHz, $\left.\mathrm{CDCl}_{3}\right) \delta$-63.0. HRMS(ESI+) Calcd. For: $\mathrm{C}_{27} \mathrm{H}_{27} \mathrm{~F}_{6} \mathrm{FeNO}_{2}\left([\mathrm{M}]^{+}\right): 567.1281$, found: 567.1280. The product was analyzed by HPLC to determine the enantiomeric excess: $97 \%$ ee $($ Chiralpak AD-H, $i$-propanol/hexane $=10 / 90$, flow rate $1.0 \mathrm{~mL} / \mathrm{min}, \lambda=254 \mathrm{~nm})$; retention times: $\mathrm{t}_{1}$ $=5.64 \min$ (major), $\mathrm{t}_{2}=8.73 \min$ (minor) .

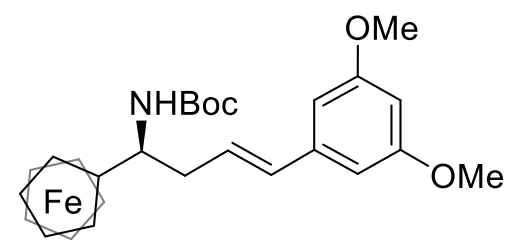

\section{tert-butyl $(S, E)-(4-(3,5-d i m e t h o x y p h e n y l)-1-f e r r o c e n y l b u t-3-e n-1-y l)$ carbamate (15e')}

Yield (211mg, 86\%); yellow solid; m.p. $=78-80{ }^{\circ} \mathrm{C} ;[\alpha]^{28} \mathrm{D}=+16.2\left(c 0.11, \mathrm{CHCl}_{3}\right) ;{ }^{1} \mathrm{H}$ NMR $(400$ $\left.\mathrm{MHz}, \mathrm{CDCl}_{3}\right) \delta 6.50(\mathrm{~d}, J=2.2 \mathrm{~Hz}, 2 \mathrm{H}), 6.41-6.30(\mathrm{~m}, 2 \mathrm{H}), 6.25-6.10(\mathrm{~m}, 1 \mathrm{H}), 4.66(\mathrm{~m}, 1 \mathrm{H})$, $4.18(\mathrm{~s}, 5 \mathrm{H}), 4.16-4.12(\mathrm{~m}, 4 \mathrm{H}), 3.78(\mathrm{~s}, 6 \mathrm{H}), 2.72-2.65(\mathrm{~m}, 1 \mathrm{H}), 2.52-2.48(\mathrm{~m}, 1 \mathrm{H}), 1.46(\mathrm{~s}, 9 \mathrm{H})$. ${ }^{13} \mathrm{C} \mathrm{NMR}\left(101 \mathrm{MHz}, \mathrm{CDCl}_{3}\right) \delta 160.8,155.2,139.5,132.4,127.0,104.2,99.5,90.9,79.3,68.7,67.7$, 67.6, 66.9, 66.1, 55.3, 49.3, 40.4, 28.4. HRMS(ESI+) Calcd. For: $\mathrm{C}_{27} \mathrm{H}_{33} \mathrm{FeNO}_{4}\left([\mathrm{M}]^{+}\right)$: 491.1754, found: 491.1745 . The product was analyzed by HPLC to determine the enantiomeric excess: $98 \% e e$ 
$($ Chiralpak AD-H, $i$-propanol/hexane $=10 / 90$, flow rate $1.0 \mathrm{~mL} / \mathrm{min}, \lambda=220 \mathrm{~nm})$; retention times: $\mathrm{t}_{1}$ $=15.95 \min$ (major), $\mathrm{t}_{2}=20.78 \mathrm{~min}$ (minor).<smiles>NC(CCC(=O)O)c1ccccc1</smiles>

tert-butyl $(S, E)$-(1-ferrocenyl-4-(furan-2-yl)but-3-en-1-yl)carbamate (15f')

Yield $(170 \mathrm{mg}, 81 \%)$; yellow oil; $[\alpha]^{28}{ }_{\mathrm{D}}=+27.1\left(c\right.$ 0.11, $\left.\mathrm{CHCl}_{3}\right) ;{ }^{1} \mathrm{H} \mathrm{NMR}\left(400 \mathrm{MHz}, \mathrm{CDCl}_{3}\right) \delta$ $7.29(\mathrm{~d}, J=1.8 \mathrm{~Hz}, 1 \mathrm{H}), 6.33(\mathrm{dd}, J=3.3,1.8 \mathrm{~Hz}, 1 \mathrm{H}), 6.25(\mathrm{~d}, J=15.9 \mathrm{~Hz}, 1 \mathrm{H}), 6.16(\mathrm{dd}, J=10.2$, $5.0 \mathrm{~Hz}, 2 \mathrm{H}), 4.72-4.57(\mathrm{~m}, 1 \mathrm{H}), 4.17(\mathrm{~s}, 5 \mathrm{H}), 4.13(\mathrm{~m}, 4 \mathrm{H}), 2.69-2.60(\mathrm{~m}, 1 \mathrm{H}), 2.53-2.46$ $(\mathrm{m}, 1 \mathrm{H}), 1.46(\mathrm{~s}, 9 \mathrm{H}) .{ }^{13} \mathrm{C} \mathrm{NMR}\left(101 \mathrm{MHz}, \mathrm{CDCl}_{3}\right) \delta 155.2,152.9,141.5,125.2,121.1,111.1,106.6$, 90.9, 79.3, 68.7, 67.7, 67.6, 66.8, 66.3, 49.3, 40.1, 28.4. HRMS(ESI+) Calcd. For: $\mathrm{C}_{23} \mathrm{H}_{27} \mathrm{FeNO}_{3}$ $\left([\mathrm{M}]^{+}\right)$: 421.1335, found: 421.1332. The product was analyzed by HPLC to determine the enantiomeric excess: > 99\% ee (Chiralpak AD-H, $i$-propanol $/$ hexane $=10 / 90$, flow rate $1.0 \mathrm{~mL} / \mathrm{min}$, $\lambda=254 \mathrm{~nm}$ ); retention times: $\mathrm{t}_{1}=10.63 \min$ (major), $\mathrm{t}_{2}=15.71 \mathrm{~min}$ (minor).

\section{Practical Preparation of Enantioenriched Ugi-Type Amine Derivative (S)-15 and (S)-16}

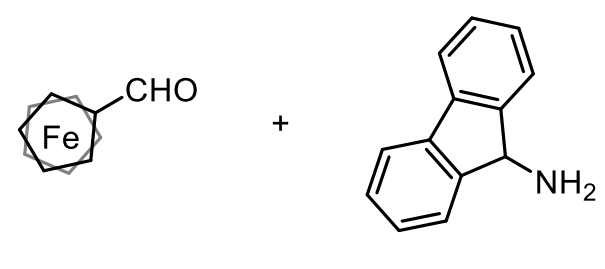

13

14

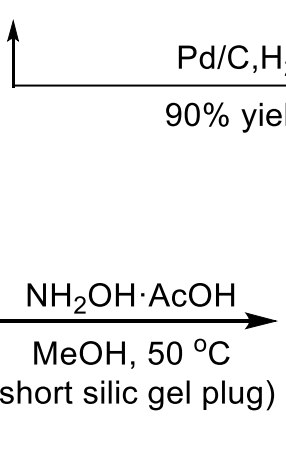

$(S)-12 a, 90 \%$ yield (after recrystallization)

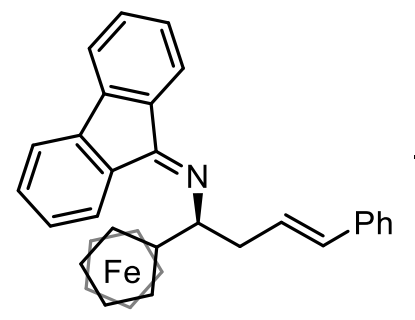

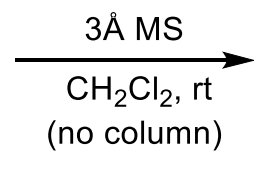

9-fluorenone oxime

$14 ', 97 \%$ yield

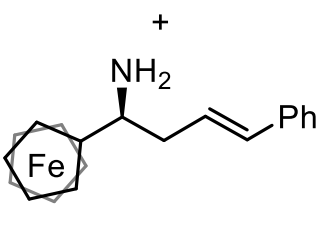

(S)-15a, 98\% yield<smiles>C(=N/C1c2ccccc2-c2ccccc21)\c1ccccc1</smiles>

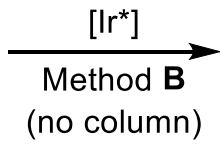

$11,>99 \%$ yield

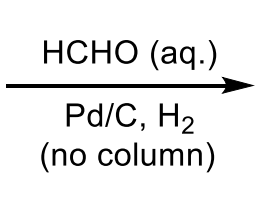

(S)-16, 85\% yield (after recrystallization)

Scheme S1. Practical Preparation of Enantioenriched Long-Chained Ugi-Type Amine (S)-15 and $(S)-16$ 
To a suspension of 9-aminofluorene $14(18.1 \mathrm{~g}, 100 \mathrm{mmol})$ and $3 \AA \mathrm{MS}(75 \mathrm{~g})$ in $\mathrm{CH}_{2} \mathrm{Cl}_{2}(200$ $\mathrm{mL})$ was added ferrocenecarboxaldehyde $\mathbf{1 3}(21.4 \mathrm{~g}, 100 \mathrm{mmol})$ in $\mathrm{CH}_{2} \mathrm{Cl}_{2}(50 \mathrm{~mL})$ via syringe. The reaction mixture was stirred at room temperature until complete consumption of starting material. The reaction was diluted with $200 \mathrm{~mL} \mathrm{CH}_{2} \mathrm{Cl}_{2}$, and then the resulting precipitate was removed by filtration. The residue was washed with $200 \mathrm{~mL} \mathrm{CH}_{2} \mathrm{Cl}_{2}$ five times, and the combined organic phase was washed with brine, dried over $\mathrm{MgSO}_{4}$ and concentrated to get the resulting aldimine 11 (37.3 $\mathrm{g},>99 \%$ yield) which was used directly without further purification.

A flame dried Schlenk tube was cooled to room temperature and filled with $\mathrm{N}_{2}$. To this flask were added $[\operatorname{Ir}(\mathrm{COD}) \mathrm{Cl}]_{2}$ (1 eq.), phosphoramidite ligand $(S, S, S a)-\mathbf{L} 2$ (2 eq.), $50 \mathrm{~mL}$ degassed THF, degassed $n$-propylamine $(25 \mathrm{~mL})$. The reaction mixture was heated at $50{ }^{\circ} \mathrm{C}$ for $30 \mathrm{~min}$ and then the volatile solvent was removed under vacuum to give the iridium complex as a pale yellow solid. To $500 \mathrm{~mL}$ three-necked flask was added aldimine 11 (37.3 g, 99 mmol), new made iridium complex (3.1g, $3 \mathrm{mmol})$ and cinnamyl carbonate 9a $(20.2 \mathrm{~g}, 105 \mathrm{mmol})$ in THF (100 mL), then DBU (15.2 g, $100 \mathrm{mmol})$ in THF (20 mL) were added dropwise. The reaction was then stirred at room temperature until complete consumption of starting material 11 (detected by TLC). Then THF was removed under vacuum to give dark brown oil which was diluted with petroleum ether and ethyl acetate (PE: EA = 1:1). The mixture was filtrated with bergmeal and washed with petroleum ether and ethyl acetate (PE: $\mathrm{EA}=1: 1)$. Then the volatile solvent was removed under vacuum and enantiopure $(S)-\mathbf{1 2 a}$ could be recrystallized with petroleum ether and ethyl acetate $(44.0 \mathrm{~g}, 90 \%$ yield, >99\% ee).

We also tried to reduce iridium catalyst loading to $2 \mathrm{~mol} \%$ and $1 \mathrm{~mol} \%$ under otherwise standard condition at 10-gram scale and the results are summarized as below (Table S1). Using 2 mol \% iridium catalyst, it took 24 hours to complete the consumption of starting material 11 (detected by TLC) and got (S)-12a (4.63 g, 94\% yield, 97\% ee); while using 1 mol \% iridium catalyst, it took 40 hours to complete the consumption of starting material $\mathbf{1 1}$ (detected by TLC) and got $(S)-12 a(4.52 \mathrm{~g}, 92 \%$ yield, $96 \%$ ee $)$. 
Table S1. Catalytic Asymmetric Synthesis of $(S)$-12a at Reduced Iridium Catalyst Loading

\begin{tabular}{ccccc}
\hline entry & {$[$ Ir-complex] } & Time $(\mathrm{h})$ & ${\text { Yield }(\%)^{a}}^{a}$ & Ee $(\%)^{b}$ \\
\hline $\mathbf{1}$ & $1 \mathrm{~mol} \%$ & 40 & 92 & 97 \\
\hline $\mathbf{2}$ & $2 \mathrm{~mol} \mathrm{\%}$ & 24 & 94 & 96 \\
\hline
\end{tabular}

${ }^{a}$ Isolate yield. ${ }^{\mathrm{b}}$ Determined by HPLC analysis.

To $(S)$-12a $(44 \mathrm{~g}, 89.1 \mathrm{mmol})$ in $\mathrm{MeOH}(150 \mathrm{~mL})$ was added $\mathrm{NH}_{2} \mathrm{OH} \cdot \mathrm{AcOH}(12.5 \mathrm{~g}, 134 \mathrm{mmol})$ and then stirred for 2 hours at $50{ }^{\circ} \mathrm{C}$, after which the crude reaction mixture was removed under vacuum and the remaining was directly subjected to a short column chromatography purification (from PE:EA = 1:1 to $\left.\mathrm{EA}: \mathrm{Et}_{3} \mathrm{~N}=1: 5 \%\right)$ to get 9-fluorenone oxime $(16.9 \mathrm{~g}, 97 \%$ yield) and $(S)-15 \mathbf{a}$ (28.9 g, 98\% yield).

9-fluorenone oxime (16.9 g, $86 \mathrm{mmol})$ in $\mathrm{MeOH}(80 \mathrm{~mL})$ was added $5 \mathrm{wt} \% \mathrm{Pd} / \mathrm{C}$ and acetic acid (7.8 g, $130 \mathrm{mmol}$ ) under $5 \mathrm{MPa} \mathrm{H}_{2}$ and then the reaction was stirred at $50{ }^{\circ} \mathrm{C}$ for 2 hours. the crude reaction mixture was filtrated and removed under vacuum. The residue was added $\mathrm{Et}_{3} \mathrm{~N}(36 \mathrm{~mL}, 258$ mmol) and ethyl acetate $(100 \mathrm{~mL})$. Then the mixture was washed with water $(40 \mathrm{~mL})$ three times and was removed under vacuum to get white solid. Next the solid was dissolved in enough ethyl acetate and then $12 \mathrm{~N} \mathrm{HCl}(10 \mathrm{~mL})$ was added to offer white solid which was washed five times with ethyl acetate. Then the white solid was based with $\mathrm{Et}_{3} \mathrm{~N}$ to provide $9 H$-fluoren-9-amine $\mathbf{1 4}$ as white to green solid in $90 \%$ yield.

$(S)-15 a(28.9 \mathrm{~g}, 87 \mathrm{mmol})$ in $\mathrm{MeOH}(100 \mathrm{~mL})$ was added $5 \mathrm{wt} \% \mathrm{Pd} / \mathrm{C}$ and $37 \% \mathrm{HCHO}$ solution (42 g, $522 \mathrm{mmol}$ ) under $5 \mathrm{MPa} \mathrm{H}_{2}$ in $30^{\circ} \mathrm{C}$. After complete consumption of starting material, the mixture was filtered and filter residue was washed with $\mathrm{MeOH}$. Then filtrate was concentrated to provide yellow liquid which was diluted with $\mathrm{CH}_{2} \mathrm{Cl}_{2}$. Organic phase was dried with anhydrous $\mathrm{Na}_{2} \mathrm{SO}_{4}$ and concentrated under vacuum and enantiopure $(S)$-16 could be recrystallized (26.6 g, 85\% yield). 


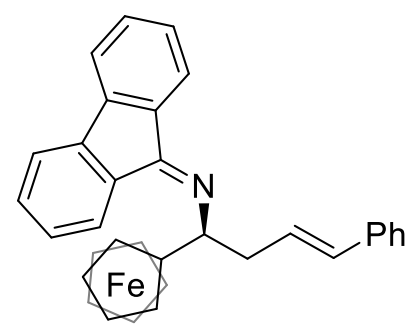

$(S, E)-N$-(1-ferrocenyl-4-phenylbut-3-en-1-yl)-9H-fluoren-9-imine (12a)

Red solid; m.p. $=116-118{ }^{\circ} \mathrm{C} ;[\alpha]^{28} \mathrm{D}=+87.3\left(c 0.15, \mathrm{CHCl}_{3}\right) ;{ }^{1} \mathrm{H}$ NMR $\left(400 \mathrm{MHz}, \mathrm{CDCl}_{3}\right) \delta 8.00$ $(\mathrm{dd}, J=12.1,7.3 \mathrm{~Hz}, 2 \mathrm{H}), 7.70(\mathrm{~d}, J=7.5 \mathrm{~Hz}, 1 \mathrm{H}), 7.62(\mathrm{~d}, J=7.4 \mathrm{~Hz}, 1 \mathrm{H}), 7.45-7.41(\mathrm{~m}, 2 \mathrm{H})$, 7.37-7.33 (m, 1H), 7.31-7.27 (m, 1H), 7.24-7.22 (m, 4H), 7.18-7.12 (m, 1H), 6.46 (d, J = 15.7 Hz, $1 \mathrm{H}), 6.24(\mathrm{dt}, J=15.6,7.2 \mathrm{~Hz}, 1 \mathrm{H}), 5.53(\mathrm{t}, J=6.6 \mathrm{~Hz}, 1 \mathrm{H}), 4.59-4.58(\mathrm{~m}, 1 \mathrm{H}), 4.18-4.15(\mathrm{~m}, 2 \mathrm{H})$, 4.12-4.10 (m, 1H), 4.06 (s, 5H), 2.95 - $2.83(\mathrm{~m}, 2 \mathrm{H}) .{ }^{13} \mathrm{C} \mathrm{NMR}\left(101 \mathrm{MHz}, \mathrm{CDCl}_{3}\right) \delta 160.1,144.0$, $140.8,139.1,137.6,132.0,131.8,131.2,130.8,128.5,128.4,128.1,127.7,127.5,127.0,126.1$, 122.8, 120.5, 119.3, 91.6, 68.6, 67.8, 67.4, 67.3, 66.9, 60.2, 42.1. HRMS(ESI+) Calcd. For: $\mathrm{C}_{33} \mathrm{H}_{27} \mathrm{FeN}\left([\mathrm{M}]^{+}\right)$: 493.1493, found: 493.1490.

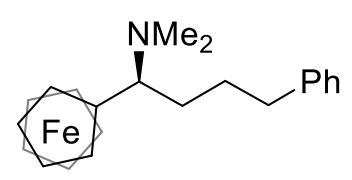

(S)-N,N-dimethyl-1-ferrocenyl-4-phenylbutan-1-amine (16)

Yellow solid; m.p. $=50-52{ }^{\circ} \mathrm{C} ;[\alpha]^{28} \mathrm{D}=-25.1\left(c 0.11, \mathrm{CHCl}_{3}\right) ;{ }^{1} \mathrm{H}$ NMR $\left(400 \mathrm{MHz}, \mathrm{CDCl}_{3}\right) \delta 7.33$ - $7.26(\mathrm{~m}, 4 \mathrm{H}), 7.21-7.14(\mathrm{~m}, 1 \mathrm{H}), 4.09-4.08$ (m, 2H), $4.04-4.03(\mathrm{~m}, 1 \mathrm{H}), 3.96$ (m, 6H), 3.32 $(\mathrm{dd}, J=10.6,3.0 \mathrm{~Hz}, 1 \mathrm{H}), 2.81-2.66(\mathrm{~m}, 2 \mathrm{H}), 1.98(\mathrm{~m}, 8 \mathrm{H}), 1.81-1.68(\mathrm{~m}, 2 \mathrm{H}) .{ }^{13} \mathrm{C} \mathrm{NMR}(101$ $\left.\mathrm{MHz}, \mathrm{CDCl}_{3}\right) \delta 142.6,128.6,128.3,125.8,85.3,69.4,68.5,67.4,67.1,66.8,63.1,40.5,35.8,30.7$, 29.1. HRMS(ESI+) Calcd. For: $\mathrm{C}_{22} \mathrm{H}_{27} \mathrm{FeN}\left([\mathrm{M}]^{+}\right)$: 361.1488 , found: 361.1481 . 


\section{Synthesis of Chiral PPFA-Type and Josiphos-Type Ligands ${ }^{1,2}$}

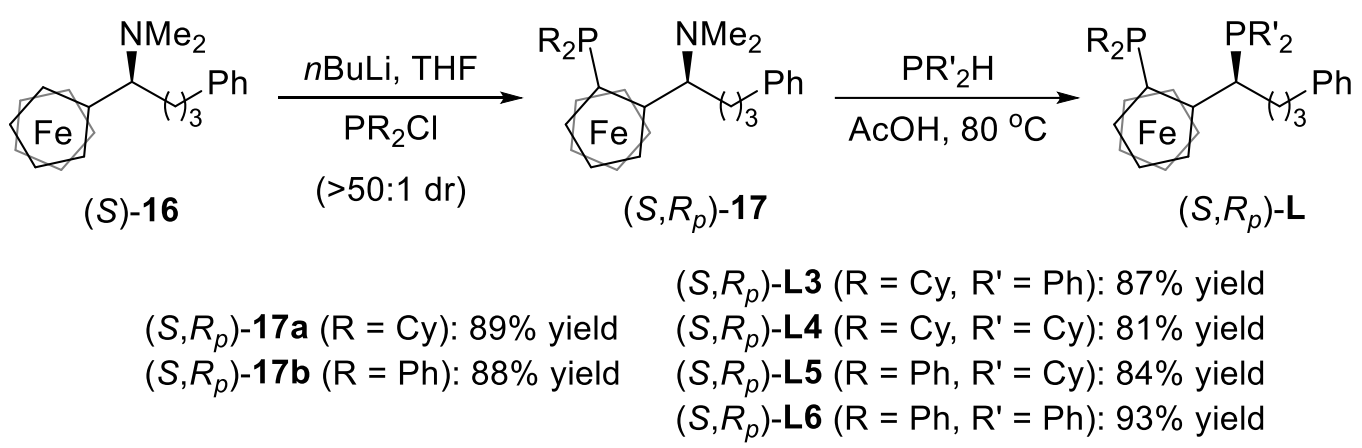

Scheme S2. Synthesis of Chiral PPFA-Type and Josiphos-Type Ligands

Under $\mathrm{N}_{2}$ condition, to $100 \mathrm{~mL}$ three-necked flask was added $(S)-16(1.8 \mathrm{~g}, 5 \mathrm{mmol})$ and $30 \mathrm{~mL}$ anhydrous $\mathrm{Et}_{2} \mathrm{O}$ with reflux condenser. Then, $2.4 \mathrm{M} n$-BuLi (3.4 mL, $\left.8.0 \mathrm{mmol}\right)$ was added dropwise with syringe pump in $1.5 \mathrm{~h}$. The reaction mixture was stirred at $25{ }^{\circ} \mathrm{C}$ for $4-6 \mathrm{~h}$. After $\mathrm{PR}_{2} \mathrm{Cl}(7.5$ mmol) in $10 \mathrm{~mL} \mathrm{Et}_{2} \mathrm{O}$ was added dropwise with syringe pump in $1.0 \mathrm{~h}$ at $38-40{ }^{\circ} \mathrm{C}$, the reaction mixture was refluxed for $16 \mathrm{~h}$. When the starting material was consumed (monitored by TLC and ${ }^{31} \mathrm{P}$ NMR spectroscopy), the reaction mixture was cooled to room temperature and quenched by adding $\mathrm{NH}_{4} \mathrm{Cl}$ aqueous solution and stirring vigorously for $5 \mathrm{~min}$. The organic layers were separated, and the aqueous layer was extracted with $\mathrm{CH}_{2} \mathrm{Cl}_{2}$. The organic layer was combined, washed with saturated brine and dried over anhydrous $\mathrm{Na}_{2} \mathrm{SO}_{4}$. The organic solvent was removed by rotary evaporation to obtain a crude mixture, which was purified by flash column chromatography to give the pure product $\left(S, R_{p}\right)-\mathbf{1 7}$.

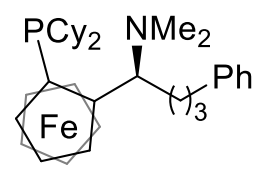

$(S)-1-\left[\left(R_{p}\right)-2-(\right.$ dicyclohexylphosphanyl)ferrocenyl)-N,N-dimethyl-4-phenylbutan-1-amine ( $S$, $\left.R_{p}\right)-17 \mathbf{a}$

Yield (2.48 g, 89\%); Orange oil; $[\alpha]^{28} \mathrm{D}=+62.9\left(c 0.11, \mathrm{CHCl}_{3}\right) ;{ }^{1} \mathrm{H} \mathrm{NMR}\left(400 \mathrm{MHz}, \mathrm{CDCl}_{3}\right) \delta 7.28$ (m, 4H), $7.21-7.15(\mathrm{~m}, 1 \mathrm{H}), 4.22-4.21(\mathrm{~m}, 1 \mathrm{H}), 4.17(\mathrm{~m}, 1 \mathrm{H}), 4.07-4.06(\mathrm{~m}, 1 \mathrm{H}), 3.92(\mathrm{~s}, 5 \mathrm{H})$, 
$3.84-3.81(\mathrm{~m}, 1 \mathrm{H}), 2.83-2.76(\mathrm{~m}, 1 \mathrm{H}), 2.69-2.62(\mathrm{~m}, 1 \mathrm{H}), 2.34-2.30(\mathrm{~m}, 1 \mathrm{H}), 2.20(\mathrm{~s}, 6 \mathrm{H})$, $2.07-1.81(\mathrm{~m}, 10 \mathrm{H}), 1.76-1.60(\mathrm{~m}, 5 \mathrm{H}), 1.34(\mathrm{~m}, 5 \mathrm{H}), 1.22-1.00(\mathrm{~m}, 5 \mathrm{H}) .{ }^{13} \mathrm{C}$ NMR $(101 \mathrm{MHz}$, $\left.\mathrm{CDCl}_{3}\right) \delta 142.3,128.5,128.3,125.8,95.8(J=21.4 \mathrm{~Hz}), 79.0(\mathrm{~J}=18.6 \mathrm{~Hz}), 70.0,69.6,68.5(J=3.5$ Hz), 67.4, $61.2(J=7.6 \mathrm{~Hz}), 40.3,36.4,36.2(J=12.3 \mathrm{~Hz}), 32.7(J=19.4 \mathrm{~Hz}), 32.3(J=16.6 \mathrm{~Hz})$, 31.1, $30.4(J=8.8 \mathrm{~Hz}), 29.3,28.9(J=6.2 \mathrm{~Hz}), 28.3(J=12.8 \mathrm{~Hz}), 27.9(J=6.7 \mathrm{~Hz}), 27.8,27.3(J=$ $7.6 \mathrm{~Hz}), 26.5(J=12.9 \mathrm{~Hz}) .{ }^{31} \mathrm{P}$ NMR $\left(162 \mathrm{MHz}, \mathrm{CDCl}_{3}\right) \delta$-12.6. HRMS(ESI+) Calcd. For $\mathrm{C}_{34} \mathrm{H}_{49} \mathrm{FeNP}\left([\mathrm{M}+\mathrm{H}]^{+}\right): 558.2947$, found: 558.2937.<smiles>CN(CCc1ccccc1)c1ccccc1</smiles>

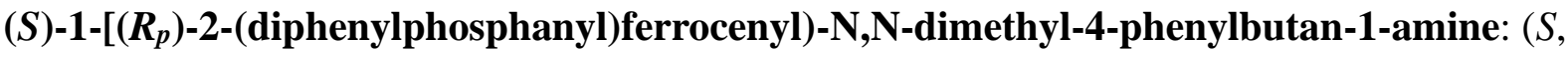
$\left.R_{p}\right) \mathbf{- 1 7 b}$

Yield (2.40 g, 88\%); Orange oil; $[\alpha]^{28} \mathrm{D}=+305.0\left(c\right.$ 0.20, $\left.\mathrm{CHCl}_{3}\right) ;{ }^{1} \mathrm{H}$ NMR $\left(400 \mathrm{MHz}, \mathrm{CDCl}_{3}\right) \delta$ $7.60-7.56(\mathrm{~m}, 2 \mathrm{H}), 7.34-7.27(\mathrm{~m}, 6 \mathrm{H}), 7.24-7.12(\mathrm{~m}, 7 \mathrm{H}), 4.24(\mathrm{~m}, 2 \mathrm{H}), 3.99-3.93(\mathrm{~m}, 1 \mathrm{H})$, $3.90(\mathrm{~d}, J=1.9 \mathrm{~Hz}, 1 \mathrm{H}), 3.78(\mathrm{~s}, 5 \mathrm{H}), 2.79(\mathrm{dt}, J=14.0,7.0 \mathrm{~Hz}, 1 \mathrm{H}), 2.69(\mathrm{dt}, J=14.1,7.5 \mathrm{~Hz}, 1 \mathrm{H})$, $2.00-1.90(\mathrm{~m}, 2 \mathrm{H}), 1.85-1.75(\mathrm{~m}, 8 \mathrm{H}) .{ }^{13} \mathrm{C} \mathrm{NMR}\left(101 \mathrm{MHz}, \mathrm{CDCl}_{3}\right) \delta 142.5,140.8(\mathrm{~J}=7.1 \mathrm{~Hz})$, $139.1(J=9.0 \mathrm{~Hz}), 135.3(J=21.7 \mathrm{~Hz}), 132.3(J=18.8 \mathrm{~Hz}), 128.8,128.6,128.4,127.9(J=7.7 \mathrm{~Hz})$, $127.4(J=6.8 \mathrm{~Hz}), 127.2,125.9,96.4(J=24.6 \mathrm{~Hz}), 76.1(J=8.6 \mathrm{~Hz}), 71.4(J=5.5 \mathrm{~Hz}), 69.7,69.6$, 68.4, $61.4(J=6.6 \mathrm{~Hz}), 39.8,36.3,30.2,29.0 .{ }^{31} \mathrm{P} \mathrm{NMR}\left(162 \mathrm{MHz}, \mathrm{CDCl}_{3}\right) \delta-23.8 . \mathrm{HRMS}(\mathrm{ESI}+)$ Calcd. For $\mathrm{C}_{34} \mathrm{H}_{37} \mathrm{FeNP}\left([\mathrm{M}+\mathrm{H}]^{+}\right)$: 546.2008, found: 546.1996 .

Under $\mathrm{N}_{2}$ condition, to $50 \mathrm{~mL}$ flame dried Schlenk tube was added $\left(S, R_{p}\right)-\mathbf{1 7}(2 \mathrm{mmol})$ and 25 $\mathrm{mL}$ degassed $\mathrm{AcOH}$, and then $\mathrm{PR}_{2}^{\prime} \mathrm{H}$ were added into this flask via syringe. The reaction mixture was stirred at $85{ }^{\circ} \mathrm{C}$ overnight. When the starting material was consumed (monitored by TLC), the reaction mixture was cooled to room temperature and quenched by adding $\mathrm{NH}_{4} \mathrm{Cl}$ aqueous solution and stirring vigorously for $5 \mathrm{~min}$. The organic layers were separated, and the aqueous layer was extracted with $\mathrm{CH}_{2} \mathrm{Cl}_{2}$. The organic layer was combined, washed with saturated brine and dried over anhydrous $\mathrm{Na}_{2} \mathrm{SO}_{4}$. The organic solvent was removed by rotary evaporation to obtain a crude 
mixture, which was purified by flash column chromatography and recrystallized with degassed $\mathrm{MeOH}$ to give the pure product $\left(S, R_{p}\right)-\mathbf{L}$.

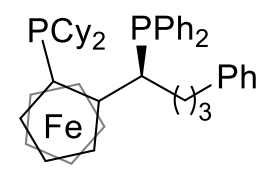

(S)-1-[( $\left.\boldsymbol{R}_{p}\right)$-2-(dicyclohexylphosphino)ferrocenyl]-4-phenylbutyldiphenylphosphine: $\left(S, R_{p}\right)$-L3 Yield $(1.21 \mathrm{~g}, 87 \%)$; Orange solid; m.p. $=76-78{ }^{\circ} \mathrm{C} ;[\alpha]^{28} \mathrm{D}=+160.3\left(c 0.11, \mathrm{CHCl}_{3}\right) ;{ }^{1} \mathrm{H}$ NMR $\left(400 \mathrm{MHz}, \mathrm{CDCl}_{3}\right) \delta 7.50(\mathrm{~m}, 2 \mathrm{H}), 7.39-7.25(\mathrm{~m}, 5 \mathrm{H}), 7.21-7.19(\mathrm{~m}, 2 \mathrm{H}), 7.16-7.12(\mathrm{~m}, 4 \mathrm{H})$, $7.06-7.03(\mathrm{~m}, 2 \mathrm{H}), 4.26-4.24(\mathrm{~m}, 1 \mathrm{H}), 4.17-4.15(\mathrm{~m}, 1 \mathrm{H}), 4.12-4.09(\mathrm{~m}, 1 \mathrm{H}), 4.05(\mathrm{~s}, 5 \mathrm{H})$, $3.33(\mathrm{~m}, 1 \mathrm{H}), 2.49-2.47(\mathrm{~m}, 1 \mathrm{H}), 2.39-2.37(\mathrm{~m}, 1 \mathrm{H}), 2.19-2.06(\mathrm{~m}, 3 \mathrm{H}), 2.06-1.62(\mathrm{~m}, 10 \mathrm{H})$, $1.58-1.52(\mathrm{~m}, 2 \mathrm{H}), 1.49-1.35(\mathrm{~m}, 3 \mathrm{H}), 1.32-1.08(\mathrm{~m}, 6 \mathrm{H}), 1.02-0.82(\mathrm{~m}, 2 \mathrm{H}) .{ }^{13} \mathrm{C}$ NMR $(101$ $\left.\mathrm{MHz}, \mathrm{CDCl}_{3}\right) \delta 142.4,138.3(\mathrm{~d}, J=17.4 \mathrm{~Hz}), 136.9(\mathrm{~d}, J=18.0 \mathrm{~Hz}), 135.1(\mathrm{~d}, J=21.1 \mathrm{~Hz}), 132.6$ $(\mathrm{d}, J=17.0 \mathrm{~Hz}), 129.0,128.4,128.2,128.1(\mathrm{~d}, J=7.5 \mathrm{~Hz}), 127.8(\mathrm{~d}, J=5.6 \mathrm{~Hz}), 127.5,125.5,100.1$ $(\mathrm{dd}, J=24.3,19.4 \mathrm{~Hz}), 79.7(\mathrm{dd}, J=22.8,3.4 \mathrm{~Hz}), 70.7(\mathrm{~d}, J=2.9 \mathrm{~Hz}), 69.3,68.9(\mathrm{dd}, J=15.4,4.2$ $\mathrm{Hz}), 67.4,37.5(\mathrm{~d}, J=13.0 \mathrm{~Hz}), 37.2(\mathrm{dd}, J=11.6,1.4 \mathrm{~Hz}), 36.1,34.9(\mathrm{dd}, J=11.4,2.1 \mathrm{~Hz}), 33.9$ $(\mathrm{dd}, J=16.3,8.9 \mathrm{~Hz}), 33.3(\mathrm{~d}, J=23.8 \mathrm{~Hz}), 31.1(\mathrm{~m}), 29.4(\mathrm{~d}, J=2.7 \mathrm{~Hz}), 28.2(\mathrm{~d}, J=15.6 \mathrm{~Hz})$, 27.5, $27.4(\mathrm{~d}, J=5.6 \mathrm{~Hz}), 27.1(\mathrm{~d}, J=10.0 \mathrm{~Hz}), 26.4(\mathrm{~d}, J=8.4 \mathrm{~Hz}) .{ }^{31} \mathrm{P} \mathrm{NMR}\left(162 \mathrm{MHz}, \mathrm{CDCl}_{3}\right) \delta$ $1.8(\mathrm{~d}, J=5.1 \mathrm{~Hz}),-16.1(\mathrm{~d}, J=5.1 \mathrm{~Hz}) . \mathrm{HRMS}(\mathrm{ESI}+)$ Calcd. For $\mathrm{C}_{44} \mathrm{H}_{53} \mathrm{FeNP}_{2}\left([\mathrm{M}+\mathrm{H}]^{+}\right): 699.2966$, found: 699.2962 .

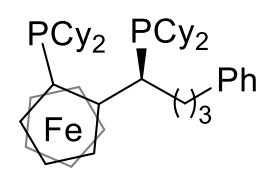

$(S)-1-\left[\left(R_{p}\right)-2-(\right.$ dicyclohexylphosphino)ferrocenyl]-4-phenylbutyldicyclohexylphosphine: $(S$, $\left.R_{p}\right) \mathbf{- L 4}$

Yield $(1.14 \mathrm{~g}, 81 \%)$; Orange solid; m.p. $=88-90{ }^{\circ} \mathrm{C} ;[\alpha]^{28} \mathrm{D}=+143.6\left(c 0.13, \mathrm{CHCl}_{3}\right) ;{ }^{1} \mathrm{H} \mathrm{NMR}$ $\left(400 \mathrm{MHz}, \mathrm{CDCl}_{3}\right) \delta 7.31-7.25(\mathrm{~m}, 4 \mathrm{H}), 7.20-7.15(\mathrm{~m}, 1 \mathrm{H}), 4.34(\mathrm{~s}, 1 \mathrm{H}), 4.23(\mathrm{t}, J=2.5 \mathrm{~Hz}, 1 \mathrm{H})$, $4.14(\mathrm{t}, J=1.8 \mathrm{~Hz}, 1 \mathrm{H}), 4.05(\mathrm{~s}, 5 \mathrm{H}), 2.78-2.67(\mathrm{~m}, 3 \mathrm{H}), 2.20-1.95(\mathrm{~m}, 6 \mathrm{H}), 1.90-1.50(\mathrm{~m}, 20 \mathrm{H})$, $1.46-1.33(\mathrm{~m}, 4 \mathrm{H}), 1.28-1.04(\mathrm{~m}, 18 \mathrm{H}) .{ }^{13} \mathrm{C} \mathrm{NMR}\left(101 \mathrm{MHz}, \mathrm{CDCl}_{3}\right) \delta 142.5,128.6,128.3,125.7$, 
101.8, (dd, $J=24.2,12.9 \mathrm{~Hz}), 80.0(\mathrm{~d}, J=23.4 \mathrm{~Hz}), 70.8,70.3,69.2,68.6(\mathrm{dd}, J=10.6,4.1 \mathrm{~Hz})$, 67.3, $38.1(\mathrm{~d}, J=13.6 \mathrm{~Hz}), 37.3(\mathrm{~d}, J=11.5 \mathrm{~Hz}), 36.5,34.8(\mathrm{dd}, J=11.5,2.9 \mathrm{~Hz}), 33.4(\mathrm{~d}, J=29.8$ $\mathrm{Hz}), 33.1(\mathrm{~d}, J=25.0 \mathrm{~Hz}), 32.3(\mathrm{~d}, J=4.0 \mathrm{~Hz}), 32.1(\mathrm{~d}, J=8.6 \mathrm{~Hz}), 31.6-31.3(\mathrm{~m}), 31.1-30.9(\mathrm{~m})$, $29.8(\mathrm{dd}, J=22.2,8.7 \mathrm{~Hz}), 29.5,28.3(\mathrm{~d}, J=15.6 \mathrm{~Hz}), 27.9,27.8(\mathrm{~d}, J=2.8 \mathrm{~Hz}), 27.7-27.4(\mathrm{~m})$, 27.3 - $27.1(\mathrm{~m}), 26.6$ - $26.4(\mathrm{~m}) .{ }^{31} \mathrm{P}$ NMR (162 MHz, $\left.\mathrm{CDCl}_{3}\right) \delta 17.9,-15.5$. HRMS(ESI+) Calcd. For $\mathrm{C}_{44} \mathrm{H}_{63} \mathrm{FeNP}_{2}\left([\mathrm{M}+\mathrm{H}]^{+}\right):$711.3905, found: 711.3901 .

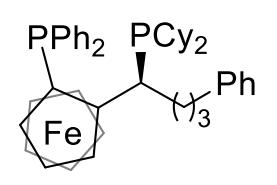

(S)-1-[( $\left.\boldsymbol{R}_{p}\right)$-2-(diphenylphosphino)ferrocenyl]-4-phenylbutyldicyclohexylphosphine: $\left(S, R_{p}\right)$-L5 Yield (1.17 g, 84\%); Orange solid; m.p. $=84-86{ }^{\circ} \mathrm{C}[\alpha]^{28} \mathrm{D}=+202.8\left(c 0.11, \mathrm{CHCl}_{3}\right) ;{ }^{1} \mathrm{H}$ NMR $(400$ $\left.\mathrm{MHz}, \mathrm{CDCl}_{3}\right) \delta 7.62(\mathrm{~m}, 2 \mathrm{H}), 7.36-7.28(\mathrm{~m}, 9 \mathrm{H}), 7.23-7.16(\mathrm{~m}, 4 \mathrm{H}), 4.35(\mathrm{t}, J=2.6 \mathrm{~Hz}, 1 \mathrm{H})$, $4.30-4.28(\mathrm{~m}, 1 \mathrm{H}), 4.16-4.15(\mathrm{~m}, 1 \mathrm{H}), 3.63(\mathrm{~s}, 5 \mathrm{H}), 2.92-2.77(\mathrm{~m}, 2 \mathrm{H}), 2.72-2.67(\mathrm{~m}, 1 \mathrm{H}), 2.32$ - $2.14(\mathrm{~m}, 2 \mathrm{H}), 2.06-1.98(\mathrm{~m}, 1 \mathrm{H}), 1.84-1.77(\mathrm{~m}, 1 \mathrm{H}), 1.67-1.44(\mathrm{~m}, 9 \mathrm{H}), 1.31-1.21(\mathrm{~m}, 3 \mathrm{H})$, $1.10-0.93(\mathrm{~m}, 10 \mathrm{H}) .{ }^{13} \mathrm{C}$ NMR $\left(101 \mathrm{MHz}, \mathrm{CDCl}_{3}\right) \delta 142.6,141.1(\mathrm{~d}, J=8.1 \mathrm{~Hz}), 139.6(\mathrm{~d}, J=8.9$ Hz), $135.6(\mathrm{~d}, J=23.1 \mathrm{~Hz}), 132.7(\mathrm{~d}, J=17.9 \mathrm{~Hz}), 128.9,128.7,128.3,127.8$ (d, $J=8.5 \mathrm{~Hz}), 127.6$ $(\mathrm{d}, J=6.0 \mathrm{~Hz}), 127.3,125.8,103.2(\mathrm{dd}, J=31.3,3.9 \mathrm{~Hz}), 73.5(\mathrm{~d}, J=11.9 \mathrm{~Hz}), 70.7(\mathrm{~d}, J=4.5 \mathrm{~Hz})$, 69.7, 69.4, 69.1, 36.7 (d, $J=18.2 \mathrm{~Hz}), 36.2,32.9(\mathrm{~d}, J=20.1 \mathrm{~Hz}), 32.3(\mathrm{~d}, J=19.7 \mathrm{~Hz}), 31.5-31.4$ (m), $31.0(\mathrm{~d}, J=13.7 \mathrm{~Hz}), 30.8-30.4(\mathrm{~m}), 29.8(\mathrm{~d}, J=6.2 \mathrm{~Hz}), 27.7(\mathrm{~d}, J=12.7 \mathrm{~Hz}), 27.4(\mathrm{~d}, J=$ $6.4 \mathrm{~Hz}), 26.7(\mathrm{~d}, J=2.8 \mathrm{~Hz}), 26.6(\mathrm{~d}, J=6.9 \mathrm{~Hz}) .26 .3(\mathrm{~d}, J=8.9 \mathrm{~Hz}){ }^{31} \mathrm{P} \mathrm{NMR}\left(162 \mathrm{MHz}, \mathrm{CDCl}_{3}\right) \delta$ $21.5(\mathrm{~d}, J=10.0 \mathrm{~Hz}),-26.2(\mathrm{~d}, J=10.0 \mathrm{~Hz})$. HRMS(ESI+) Calcd. For $\mathrm{C}_{44} \mathrm{H}_{53} \mathrm{FeNP}_{2}\left([\mathrm{M}+\mathrm{H}]^{+}\right)$: 699.2966, found: 699.2961 .

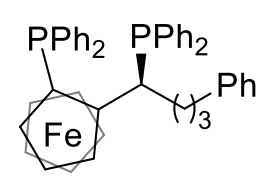

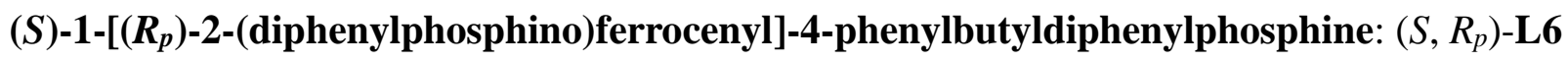
Yield (1.27 g, 93\%); Orange solid; m.p. $=92-94{ }^{\circ} \mathrm{C} ;[\alpha]^{28} \mathrm{D}=+309.2\left(c 0.16, \mathrm{CHCl}_{3}\right) ;{ }^{1} \mathrm{H}$ NMR $\left(400 \mathrm{MHz} \mathrm{CDCl}_{3}\right) \delta 7.61-7.57(\mathrm{~m}, 2 \mathrm{H}), 7.43(\mathrm{~m}, 2 \mathrm{H}), 7.37-7.32(\mathrm{~m}, 3 \mathrm{H}), 7.32-7.01(\mathrm{~m}, 19 \mathrm{H})$, 
$4.30-4.26(\mathrm{~m}, 1 \mathrm{H}), 4.24(\mathrm{t}, J=2.5 \mathrm{~Hz}, 1 \mathrm{H}), 3.99(\mathrm{t}, J=1.7 \mathrm{~Hz}, 1 \mathrm{H}), 3.72(\mathrm{~s}, 5 \mathrm{H}), 3.48-3.43(\mathrm{~m}$, $1 \mathrm{H}), 2.56-2.49(\mathrm{~m}, 1 \mathrm{H}), 2.43-2.36(\mathrm{~m}, 1 \mathrm{H}), 2.21-2.08(\mathrm{~m}, 1 \mathrm{H}), 1.96-1.80(\mathrm{~m}, 2 \mathrm{H}), 1.62-1.47$ $(\mathrm{m}, 1 \mathrm{H}) .{ }^{13} \mathrm{C} \mathrm{NMR}\left(101 \mathrm{MHz}, \mathrm{CDCl}_{3}\right) \delta 142.3,140.1(\mathrm{~d}, J=8.0 \mathrm{~Hz}), 139.5(\mathrm{~d}, J=9.2 \mathrm{~Hz}), 137.6(\mathrm{~d}$, $J=17.4 \mathrm{~Hz}), 136.7(\mathrm{~d}, J=19.1 \mathrm{~Hz}), 135.3(\mathrm{~d}, J=22.5 \mathrm{~Hz}), 134.7(\mathrm{~d}, J=20.9 \mathrm{~Hz}), 132.7(\mathrm{~d}, J=$ $17.9 \mathrm{~Hz}), 132.5(\mathrm{dd}, J=17.3,1.2 \mathrm{~Hz}), 128.9$ (d, $J=6.3 \mathrm{~Hz}), 128.4,128.2,128.1,128.0(\mathrm{~d}, J=1.0$ Hz), 127.9, 127.8, 127.7, 127.6 (d, $J=5.8 \mathrm{~Hz}), 127.2$, 125.6, 100.9 (dd, $J=28.0,20.2 \mathrm{~Hz}), 75.5(\mathrm{dd}$, $J=11.0,3.4 \mathrm{~Hz}), 70.5(\mathrm{~d}, J=4.5 \mathrm{~Hz}), 70.0(\mathrm{dd}, J=12.7,4.9 \mathrm{~Hz}), 69.5,68.6,36.3(\mathrm{~d}, J=1.6 \mathrm{~Hz})$, $36.1(\mathrm{~d}, J=0.7 \mathrm{~Hz}), 34.5(\mathrm{dd}, J=17.3,9.0 \mathrm{~Hz}), 31.2(\mathrm{dd}, J=5.8,2.1 \mathrm{~Hz}) .{ }^{31} \mathrm{P}$ NMR $(162 \mathrm{MHz}$, $\left.\mathrm{CDCl}_{3}\right) \delta 2.8(\mathrm{~d}, J=14.5 \mathrm{~Hz}),-25.7(\mathrm{~d}, J=14.5 \mathrm{~Hz})$. HRMS(ESI+) Calcd. For $\mathrm{C}_{44} \mathrm{H}_{41} \mathrm{FeNP}_{2}$ $\left([\mathrm{M}+\mathrm{H}]^{+}\right): 687.2027$, found: 687.2014 .

\section{7. $\mathrm{Cu}(\mathrm{I}) /\left(S, R_{p}\right)$-17b-Catalyzed 1,3-Dipolar Cycloaddition Reactions of Azomethine Ylides}

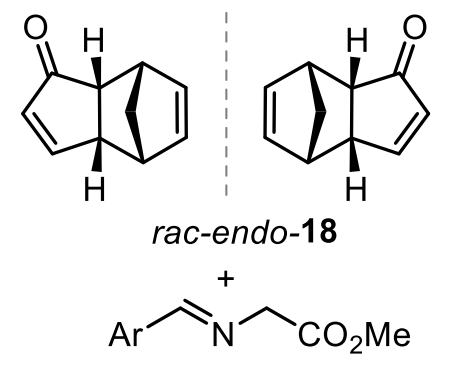

$$
\left(19, \mathrm{Ar}=p-\mathrm{Cl}-\mathrm{C}_{6} \mathrm{H}_{4}\right)
$$

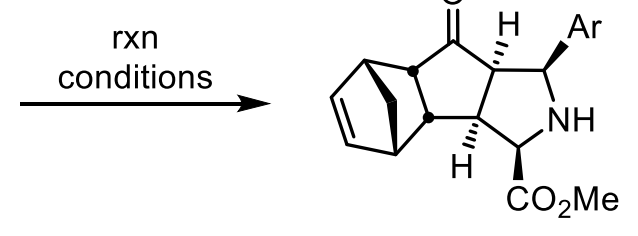

endo-endo-20

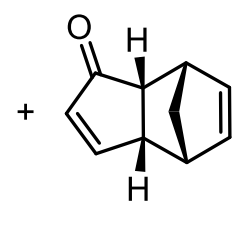

$(-)-18$

reaction conditions: $\mathrm{Cu}(\mathrm{MeCN})_{4} \mathrm{BF}_{4} / \mathrm{L}(5 \mathrm{~mol} \%), \mathrm{DCE}, \mathrm{Et}_{3} \mathrm{~N},-30^{\circ} \mathrm{C}$<smiles>CN(CCc1ccccc1)c1ccccc1</smiles>

$$
\left(S, R_{p}\right)-\mathbf{1 7 b}
$$

(-)-18: $46 \%$ yield, $97 \%$ ee 20: $49 \%$ yield, $>20: 1 \mathrm{dr}, 97 \%$ ee $S=278$

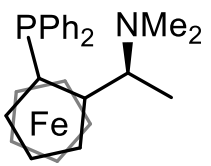

$\left(S, R_{p}\right)$-PPFA

(-)-18: $45 \%$ yield, $95 \%$ ee 20: $48 \%$ yield, $>20: 1 \mathrm{dr}, 95 \%$ ee $S=146^{[6]}$

Scheme S3. Comparative Studies of $\left(S, R_{p}\right)$-PPFA and $\left(S, R_{p}\right)$-17b in Cu(I)-Catalyzed 1,3-Dipolar Cycloaddition Reactions of Azomethine Ylides

To a $25 \mathrm{~mL}$ Schlenk tube were added $\left(S, R_{p}\right)-\mathbf{1 7 b}(0.012 \mathrm{mmol}, 6 \mathrm{~mol} \%)$ and $\mathrm{Cu}\left(\mathrm{CH}_{3} \mathrm{CN}\right)_{4} \mathrm{BF}_{4}$ $(0.01 \mathrm{mmol}, 5 \mathrm{~mol} \%)$ in $2.0 \mathrm{~mL}$ of DCE. The mixture was stirred at room temperature for $30 \mathrm{~min}$. 
After the reaction temperature was dropped to $-30{ }^{\circ} \mathrm{C}$, aldimine ester $19 \mathbf{a}(0.3 \mathrm{mmol}), \mathrm{Et}_{3} \mathrm{~N}(0.030$ mmol) and ( \pm )-endo-3-oxodicyclopentadiens $18(0.40 \mathrm{mmol})$ were added sequentially. When the reaction was completed (monitored by TLC), the organic solvent was removed and the residue was purified by column chromatography with petroleum and ethyl acetate to give the recovered (-)-18 and cycloadduct 20, which were then directly analyzed by chiral-phase GC or HPLC to determine the enantiomeric excess.<smiles>O=C1C=CC2C3C=CC(C3)C12</smiles>

((3aS,4R,7S,7aR)-3a,4,7,7a-tetrahydro-1H-4,7-methanoinden-1-one) ((-)-endo-18)

Yield (26.9 mg, 46\%), white solid, $[\alpha]^{25} \mathrm{D}=-124.5\left(c 0.58, \mathrm{CH}_{2} \mathrm{Cl}_{2}\right),{ }^{1} \mathrm{H}$ NMR $\left(400 \mathrm{MHz}, \mathrm{CDCl}_{3}\right) \delta$ $7.38(\mathrm{dd}, J=5.7,2.6 \mathrm{~Hz}, 1 \mathrm{H}), 5.97-5.93(\mathrm{~m}, 2 \mathrm{H}), 5.78(\mathrm{dd}, J=5.7,3.0 \mathrm{~Hz}, 1 \mathrm{H}), 3.44-3.40(\mathrm{~m}, 1 \mathrm{H})$, $3.23-3.21(\mathrm{~m}, 1 \mathrm{H}), 2.98-2.96(\mathrm{~m}, 1 \mathrm{H}), 2.80(\mathrm{t}, J=5.1 \mathrm{~Hz}, 1 \mathrm{H}), 1.78-1.74(\mathrm{~m}, 1 \mathrm{H}), 1.64-1.61(\mathrm{~m}$, 1H). ${ }^{13} \mathrm{C} \mathrm{NMR}\left(101 \mathrm{MHz}, \mathrm{CDCl}_{3}\right) \delta 210.7,164.6,136.9,132.6,132.4,52.7,50.2,48.3,45.0,44.0$. The product was analyzed by GC to determine the enantiomeric excess: $97 \%$ ee (Chiral Select-1000,30 m x $0.25 \mathrm{~mm}$, column temperature $180{ }^{\circ} \mathrm{C}$, carrier gas $\mathrm{N}_{2} 1.0 \mathrm{~mL} / \mathrm{min}$ ); retention times: $\mathrm{t}_{1}=5.81 \mathrm{~min}$ (major), $\mathrm{t}_{2}=6.35 \mathrm{~min}$ (minor).

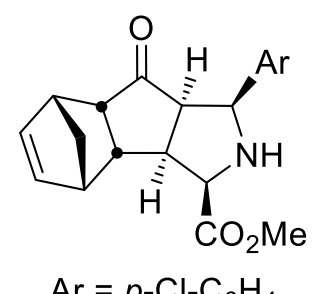

methyl $(1 S, 3 R, 3 a S, 4 S, 7 R, 8 \mathrm{a} R)-1-(4-c h l o r o p h e n y l)-8-o x o-1,2,3,3 \mathrm{a}, 3 \mathrm{~b}, 4,7,7 \mathrm{a}, 8,8 \mathrm{a}-d e c a h y d r o-4,7-$ methanoindeno[1,2-c]pyrrole-3-carboxylate: (endo-endo-20)

Yield (70.1 mg, 49\%); white solid, m.p. $154-156{ }^{\circ} \mathrm{C} ;[\alpha]^{25} \mathrm{D}=+28.6\left(c 0.49, \mathrm{CH}_{2} \mathrm{Cl}_{2}\right) ;{ }^{1} \mathrm{H}$ NMR $(400$ $\left.\mathrm{MHz}, \mathrm{CDCl}_{3}\right) \delta 7.34-7.22(\mathrm{~m}, 4 \mathrm{H}), 6.15(\mathrm{dd}, J=5.8,2.9 \mathrm{~Hz}, 1 \mathrm{H}), 6.05(\mathrm{dd}, J=5.8,2.9 \mathrm{~Hz}, 1 \mathrm{H})$, $4.34(\mathrm{~d}, J=10.1 \mathrm{~Hz}, 1 \mathrm{H}), 3.99(\mathrm{~d}, J=5.8 \mathrm{~Hz}, 1 \mathrm{H}), 3.88(\mathrm{~s}, 3 \mathrm{H}), 3.03$ - $3.00(\mathrm{~m}, 1 \mathrm{H}), 2.98$ - $2.96(\mathrm{~m}$, 1H), $2.79-2.75(\mathrm{~m}, 1 \mathrm{H}), 2.73-2.54(\mathrm{~m}, 3 \mathrm{H}), 1.52-1.49(\mathrm{~m}, 1 \mathrm{H}), 1.40-1.38(\mathrm{~m}, 1 \mathrm{H}) .{ }^{13} \mathrm{C}$ NMR 
$\left(101 \mathrm{MHz}, \mathrm{CDCl}_{3}\right) \delta 218.9,172.0,137.4,137.2,134.4,133.2,128.4,65.2,64.4,61.6,58.1,52.0$, 51.9, 46.9, 46.0, 45.7, 44.9. The product was analyzed by HPLC to determine the enantiomeric excess: $97 \%$ ee (Chiralpak AD-H, $i$-propanol $/$ hexane $=10 / 90$, flow rate $1.5 \mathrm{~mL} / \mathrm{min}, \lambda=206 \mathrm{~nm}$ ); retention times: $\mathrm{t}_{1}=14.39 \min$ (major), $\mathrm{t}_{2}=16.87 \mathrm{~min}$ (minor).

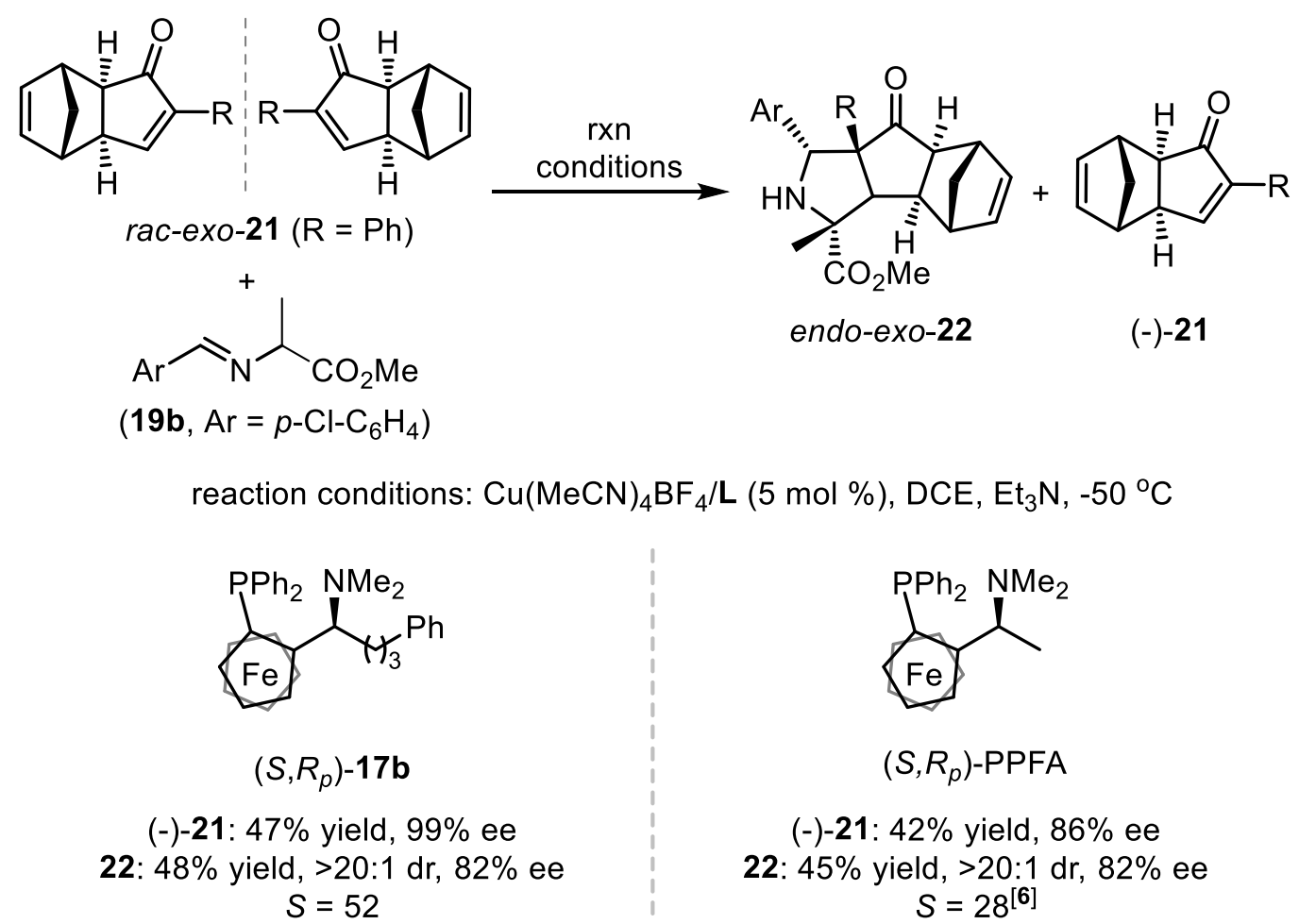

Scheme S4. Kinetic resolution of racemic exo-3-oxodicyclopentadiene via Cu-catalyzed 1,3-DCs

To a $25 \mathrm{~mL}$ Schlenk tube were added $\left(S, R_{p}\right)-\mathbf{1 7 b}(0.012 \mathrm{mmol}, 6 \mathrm{~mol} \%)$ and $\mathrm{Cu}\left(\mathrm{CH}_{3} \mathrm{CN}\right)_{4} \mathrm{BF}_{4}$ $(0.01 \mathrm{mmol}, 5 \mathrm{~mol} \%)$ in $2.0 \mathrm{~mL}$ of DCE. The mixture was stirred at room temperature for $30 \mathrm{~min}$. After the reaction temperature was dropped to $-50{ }^{\circ} \mathrm{C}$, aldimine ester $19 \mathrm{~b}(0.3 \mathrm{mmol}), \mathrm{Et}_{3} \mathrm{~N}(0.030$ mmol) and ( \pm -exo-3-oxodicyclopentadiens $21(0.40 \mathrm{mmol})$ were added sequentially. When the reaction was completed (monitored by TLC), the organic solvent was removed and the residue was purified by column chromatography with petroleum and ethyl acetate to give the recovered (-)-21 and cycloadduct 22, which were then directly analyzed by chiral-phase GC or HPLC to determine the enantiomeric excess. 


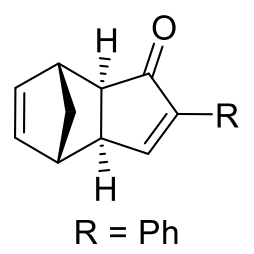

$(3 \mathrm{a} R, 4 S, 7 R, 7 \mathrm{a} R)-2-p h e n y l-3 \mathrm{a}, 4,7,7 \mathrm{a}-$ tetrahydro-1H-4,7-methanoinden-1-one: ((-)-exo-21)

yield (41.8 mg, 47\%), white solid, m.p. $84-86{ }^{\circ} \mathrm{C}[\alpha]^{25} \mathrm{D}=+9.60\left(c 0.55, \mathrm{CH}_{2} \mathrm{Cl}_{2}\right) ;{ }^{1} \mathrm{H}$ NMR $(400$ $\left.\mathrm{MHz}, \mathrm{CDCl}_{3}\right) \delta 7.69(\mathrm{~m}, 3 \mathrm{H}), 7.41-7.29(\mathrm{~m}, 3 \mathrm{H}), 6.32(\mathrm{dd}, J=5.6,3.0 \mathrm{~Hz}, 1 \mathrm{H}), 6.24(\mathrm{dd}, J=5.6$, $3.0 \mathrm{~Hz}, 1 \mathrm{H}), 3.05-2.98(\mathrm{~m}, 1 \mathrm{H}), 2.83(\mathrm{dt}, J=5.4,1.9 \mathrm{~Hz}, 1 \mathrm{H}), 2.77(\mathrm{dt}, J=3.0,1.5 \mathrm{~Hz}, 1 \mathrm{H}), 2.46$ $(\mathrm{dt}, J=5.1,1.4 \mathrm{~Hz}, 1 \mathrm{H}), 1.42(\mathrm{~m}, 1 \mathrm{H}), 1.33(\mathrm{dt}, J=9.5,1.6 \mathrm{~Hz}, 1 \mathrm{H}) .{ }^{13} \mathrm{C} \mathrm{NMR}\left(101 \mathrm{MHz}, \mathrm{CDCl}_{3}\right) \delta$ 207.7, 159.9, 147.2, 138.6, 137.2, 131.7, 128.5, 128.4, 127.1, 53.6, 47.2, 44.2, 43.4, 41.4. The product was analyzed by HPLC to determine the enantiomeric excess: 99\% ee (Chiralpak ID, $i$-propanol/hexane $=5 / 95$, flow rate $1.0 \mathrm{~mL} / \mathrm{min}, \lambda=254 \mathrm{~nm}$ ); retention times: $\mathrm{t}_{1}=10.26$ min (major), $\mathrm{t}_{2}=11.56 \min$ (minor).

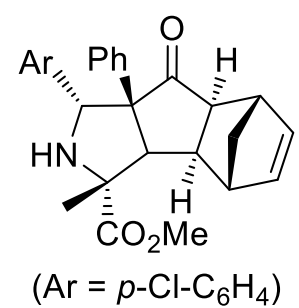

methyl (1R,3R,3bS,4R,7S,7aS,8aS)-1-(4-chlorophenyl)-3-methyl-8-oxo-8a-phenyl-1,2,3,3a,3b,4, 7,7a,8,8a-decahydro-4,7-methanoindeno[1,2-c]pyrrole-3-carboxylate: (endo-exo-22)

yield (86 mg, 48\%), white solid, m.p. $68-70{ }^{\circ} \mathrm{C},[\alpha]^{25} \mathrm{D}=+148.5\left(c 0.55, \mathrm{CH}_{2} \mathrm{Cl}_{2}\right) ;{ }^{1} \mathrm{H}$ NMR $(400$ $\left.\mathrm{MHz} \mathrm{CDCl}_{3}\right) \delta 7.56(\mathrm{~d}, J=8.4 \mathrm{~Hz}, 2 \mathrm{H}), 7.45(\mathrm{~d}, J=8.4 \mathrm{~Hz}, 2 \mathrm{H}), 7.41-7.36(\mathrm{~m}, 2 \mathrm{H}), 7.33-7.27$ (m, 2H),6.18 (dd, $J=5.6,2.8 \mathrm{~Hz}, 1 \mathrm{H}), 6.12(\mathrm{dd}, J=5.6,2.8 \mathrm{~Hz}, 1 \mathrm{H}), 4.79(\mathrm{~s}, 3 \mathrm{H}), 3.89$ (s, 3H), 3.22 $(\mathrm{d}, J=4.4,1 \mathrm{H}), 3.07-3.01(\mathrm{~m}, 1 \mathrm{H}), 2.95-2.90(\mathrm{~m}, 1 \mathrm{H}), 2.20-2.16(\mathrm{~m}, 1 \mathrm{H}), 2.03(\mathrm{dd}, J=8.4,4.4$ $\mathrm{Hz}, 1 \mathrm{H}), 1.41(\mathrm{~s}, 3 \mathrm{H}), 1.37-1.33(\mathrm{~m}, 1 \mathrm{H}), 1.13-1.08(\mathrm{~m}, 1 \mathrm{H}) .{ }^{13} \mathrm{C} \mathrm{NMR}\left(101 \mathrm{MHz}, \mathrm{CDCl}_{3}\right) \delta$ 215.0, 174.8, 141.3, 138.6, 138.4, 138.1, 133.9, 129.5, 128.6, 128.3, 127.1, 127.0, 75.2, 72.1, 70.9, $58.2,56.4,52.0,49.9,47.3,46.0,45.3,25.2$. The product was analyzed by HPLC to determine the enantiomeric excess: $82 \%$ ee (Chiralpak AD-H, $i$-propanol/hexane $=10 / 90$, flow rate $1.5 \mathrm{~mL} / \mathrm{min}, \lambda$ $=206 \mathrm{~nm}$ ); retention times: $\mathrm{t}_{1}=9.48 \min \left(\right.$ minor), $\mathrm{t}_{2}=10.53 \min$ (major). 


\section{Optimization of $\mathrm{Cu}(\mathrm{I})$-Catalyzed Asymmetric Michael Addition of Grignard Reagent 24a to $\alpha, \beta$-Unsaturated Ester $23 a^{7,8}$}

Table S2. Optimization of Cu(I)-Catalyzed Asymmetric Michael Addition of Grignard Reagent 24a to $\alpha, \beta$-Unsaturated Ester 23a ${ }^{a}$

\begin{tabular}{|c|c|c|c|c|c|}
\hline & 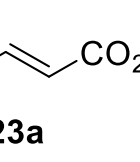 & $\begin{array}{c}\text { EtMgBr } \\
\text { 24a }\end{array}$ & $\frac{5 \mathrm{~mol} \mathrm{\%})}{{ }^{\circ} \mathrm{C}}$ & $25 a$ & \\
\hline Entry & ligand & metal precursor & solvent & Yield $(\%)^{b}$ & $\operatorname{Ee}(\%)^{c}$ \\
\hline 1 & $\mathbf{L 3}$ & $\mathrm{CuBr} \cdot \mathrm{SMe}_{2}$ & ${ }^{t} \mathrm{BuOMe}$ & 82 & 89 \\
\hline 2 & L4 & $\mathrm{CuBr} \cdot \mathrm{SMe}_{2}$ & ${ }^{t} \mathrm{BuOMe}$ & trace & - \\
\hline 3 & L5 & $\mathrm{CuBr} \cdot \mathrm{SMe}_{2}$ & ${ }^{t} \mathrm{BuOMe}$ & 60 & 63 \\
\hline 4 & L6 & $\mathrm{CuBr} \cdot \mathrm{SMe}_{2}$ & ${ }^{t} \mathrm{BuOMe}$ & trace & - \\
\hline 5 & $\mathbf{L 3}$ & $\mathrm{CuBr} \cdot \mathrm{SMe}_{2}$ & DCM & 85 & 89 \\
\hline 6 & L4 & $\mathrm{CuBr} \cdot \mathrm{SMe}_{2}$ & DCM & 33 & 89 \\
\hline 7 & L5 & $\mathrm{CuBr} \cdot \mathrm{SMe}_{2}$ & DCM & 89 & 63 \\
\hline 8 & L6 & $\mathrm{CuBr} \cdot \mathrm{SMe}_{2}$ & DCM & 90 & 49 \\
\hline 9 & $\mathbf{L 3}$ & $\mathrm{CuBr}$ & DCM & 85 & 88 \\
\hline 10 & $\mathbf{L 3}$ & $\mathrm{CuCl}$ & DCM & 80 & 90 \\
\hline 11 & $\mathbf{L 3}$ & $\mathrm{CuI}$ & DCM & 75 & 90 \\
\hline 12 & $\mathbf{L 3}$ & $\mathrm{CuOAc}$ & $\mathrm{DCM}$ & 54 & 48 \\
\hline 13 & $\mathbf{L 3}$ & $(\mathrm{CuOTf})_{2} \cdot \mathrm{Ph}$ & DCM & 71 & 82 \\
\hline 14 & $\mathbf{L 3}$ & $\mathrm{Cu}\left(\mathrm{CH}_{3} \mathrm{CN}\right)_{4} \mathrm{BF}_{4}$ & DCM & 93 & 92 \\
\hline 15 & $\mathbf{L 3}$ & $\mathrm{Cu}\left(\mathrm{CH}_{3} \mathrm{CN}\right)_{4} \mathrm{PF}_{6}$ & DCM & 80 & 90 \\
\hline 16 & $\mathbf{L 3}$ & $(\mathrm{CuOTf})_{2}$ & $\mathrm{DCM}$ & 91 & 84 \\
\hline 17 & $\mathbf{L 3}$ & $\mathrm{Cu}\left(\mathrm{CH}_{3} \mathrm{CN}\right)_{4} \mathrm{BF}_{4}$ & THF & 40 & 55 \\
\hline 18 & $\mathbf{L 3}$ & $\mathrm{Cu}\left(\mathrm{CH}_{3} \mathrm{CN}\right)_{4} \mathrm{BF}_{4}$ & DCE & 45 & 82 \\
\hline 19 & L3 & $\mathrm{Cu}\left(\mathrm{CH}_{3} \mathrm{CN}\right)_{4} \mathrm{BF}_{4}$ & toluene & 88 & 87 \\
\hline
\end{tabular}

${ }^{a}$ All reactions were carried out with $0.3 \mathrm{mmol} 23 \mathrm{a}$ and $1.5 \mathrm{mmol} 24 \mathrm{a}$ in $1 \mathrm{M}{ }^{t} \mathrm{BuOMe}, 5 \mathrm{~mol} \%[\mathrm{Cu}]$ and $6 \mathrm{~mol} \%$ ligand. ${ }^{b}$ Isolated yield. ${ }^{c}$ Ee was determined by HPLC analysis. 


\section{Scope of $\mathrm{Cu}(\mathrm{I}) /\left(S, R_{p}\right)$-L3-Catalyzed Asymmetric Michael Addition of Grignard Reagents to}

$\alpha, \beta$-Unsaturated Esters

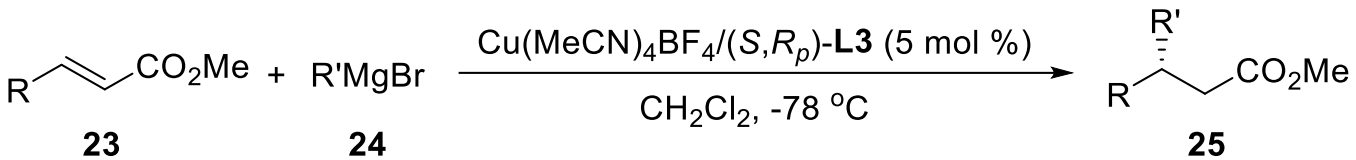

To a $25 \mathrm{~mL}$ Schlenk tube were added $\left(S, R_{p}\right)-\mathbf{L 3}(0.018 \mathrm{mmol})$ and $\mathrm{Cu}\left(\mathrm{CH}_{3} \mathrm{CN}\right)_{4} \mathrm{BF}_{4}(0.015$ $\mathrm{mmol}$ ) in $1.0 \mathrm{~mL}$ of DCM. The mixture was stirred at room temperature for $30 \mathrm{~min}$. After the reaction temperature was dropped to $-78{ }^{\circ} \mathrm{C}$, the Grignard reagents $24(1.5 \mathrm{mmol})$ in $1.5 \mathrm{~mL}$ $t$-BuOMe was dropwise added. After $15 \mathrm{~min}, \alpha, \beta$-unsaturated ester $23(0.3 \mathrm{mmol})$ in $0.5 \mathrm{~mL} \mathrm{DCM}$ was added dropwise sequentially. When the reaction was completed (monitored by TLC), the reaction was quenched by $1.5 \mathrm{~mL} \mathrm{NH}_{4} \mathrm{Cl}$ aqueous and extracted with DCM. The residue was purified by column chromatography with petroleum and diethyl ether to give the product $\mathbf{2 5}$, which were then directly analyzed by chiral-phase GC or HPLC to determine the enantiomeric excess.<smiles>CCC(CCc1ccccc1)CC(=O)OC</smiles>

\section{methyl $(S)$-3-ethyl-5-phenylpentanoate: (25a)}

Yield (61.4 mg, 93\%), colorless oil, $[\alpha]^{30}{ }_{\mathrm{D}}=-12.3\left(c\right.$ 2.0, $\left.\mathrm{CHCl}_{3}\right) ;{ }^{1} \mathrm{H}$ NMR $\left(400 \mathrm{MHz}, \mathrm{CDCl}_{3}\right) \delta$ $7.31-7.24(\mathrm{~m}, 2 \mathrm{H}), 7.21-7.14(\mathrm{~m}, 3 \mathrm{H}), 3.66(\mathrm{~s}, 3 \mathrm{H}), 2.60(\mathrm{dd}, J=9.2,7.2 \mathrm{~Hz}, 2 \mathrm{H}), 2.36-2.27(\mathrm{~m}$, 2H), $1.92-1.85(\mathrm{~m}, 1 \mathrm{H}), 1.71-1.54(\mathrm{~m}, 2 \mathrm{H}), 1.48-1.33(\mathrm{~m}, 2 \mathrm{H}), 0.90(\mathrm{t}, J=7.4 \mathrm{~Hz}, 3 \mathrm{H}) .{ }^{13} \mathrm{C}$ NMR $\left(101 \mathrm{MHz} \mathrm{CDCl}_{3}\right) \delta 172.9,141.5,127.3,127.3,124.7,50.4,37.4,35.2,34.3,32.0,25.1,9.7$. The product was analyzed by HPLC to determine the enantiomeric excess: 92\% ee (Chiralpak OD-H, $i$-propanol $/$ hexane $=0.8 / 99.2$, flow rate $1.0 \mathrm{~mL} / \mathrm{min}, \lambda=220 \mathrm{~nm}$ ); retention times: $\mathrm{t}_{1}=6.69 \min$ (major), $\mathrm{t}_{2}=7.80 \mathrm{~min}$ (minor). 
<smiles>CCC[C@H](CCc1ccccc1)CC(=O)OC</smiles>

\section{methyl (S)-3-phenethylhexanoate: $(25 b)$}

Yield (62.5 mg, 89\%); colorless oil; $[\alpha]^{30} \mathrm{D}=-7.5\left(\right.$ c 2.5, $\left.\mathrm{CHCl}_{3}\right) ;{ }^{1} \mathrm{H}$ NMR $\left(400 \mathrm{MHz}, \mathrm{CDCl}_{3}\right) \delta$ $7.29-7.25(\mathrm{~m}, 2 \mathrm{H}), 7.19-7.15(\mathrm{~m}, 3 \mathrm{H}), 3.66(\mathrm{~s}, 3 \mathrm{H}), 2.60(\mathrm{t}, J=8.2 \mathrm{~Hz}, 2 \mathrm{H}), 2.31(\mathrm{~d}, J=6.8 \mathrm{~Hz}$, 2H), $1.97-1.91(\mathrm{~m}, 1 \mathrm{H}), 1.68-1.54(\mathrm{~m}, 2 \mathrm{H}), 1.40-1.26(\mathrm{~m}, 4 \mathrm{H}), 0.89(\mathrm{t}, J=6.8,3 \mathrm{H}) .{ }^{13} \mathrm{C}$ NMR $\left(101 \mathrm{MHz}, \mathrm{CDCl}_{3}\right) \delta 173.9,142.5,128.4,128.3,125.7,51.5,38.9,36.1,35.9,34.7,33.0,19.7,14.3$. The product was analyzed by HPLC to determine the enantiomeric excess: 94\% ee (Chiralpak OD-H + OD-H, $i$-propanol $/$ hexane $=0.8 / 99.2$, flow rate $1.0 \mathrm{~mL} / \mathrm{min}, \lambda=220 \mathrm{~nm}) ;$ retention times: $\mathrm{t}_{1}=$ $11.22 \min$ (major), $\mathrm{t}_{2}=12.40 \min$ (minor).<smiles>CCCC[C@H](CCc1ccccc1)CC(=O)OC</smiles>

\section{methyl (S)-3-phenethylheptanoate: $(25 \mathrm{c})$}

Yield (65.5 mg, 88\%); colorless oil; $[\alpha]^{30} \mathrm{D}=-7.8\left(c\right.$ 2.0, $\left.\mathrm{CHCl}_{3}\right) ;{ }^{1} \mathrm{H} \mathrm{NMR}\left(400 \mathrm{MHz}, \mathrm{CDCl}_{3}\right) \delta 7.30$ $-7.23(\mathrm{~m}, 2 \mathrm{H}), 7.19-7.15(\mathrm{~m}, 3 \mathrm{H}), 3.66(\mathrm{~s}, 3 \mathrm{H}), 2.60(\mathrm{t}, J=8.2 \mathrm{~Hz}, 2 \mathrm{H}), 2.31(\mathrm{~d}, J=6.8 \mathrm{~Hz}, 2 \mathrm{H})$, $1.96-1.89(\mathrm{~m}, 1 \mathrm{H}), 1.70-1.54(\mathrm{~m}, 2 \mathrm{H}), 1.42-1.23(\mathrm{~m}, 6 \mathrm{H}), 0.94-0.85(\mathrm{~m}, 3 \mathrm{H}) .{ }^{13} \mathrm{C}$ NMR $(101$ $\left.\mathrm{MHz}, \mathrm{CDCl}_{3}\right) \delta 173.9,142.5,128.4,128.3,125.7,51.5,38.9,35.9,34.9,33.4,33.0,28.7,22.9,14.1$. The product was analyzed by HPLC to determine the enantiomeric excess: 97\% ee (Chiralpak OD-H + OD-H, $i$-propanol/hexane $=0.8 / 99.2$, flow rate $1.0 \mathrm{~mL} / \mathrm{min}, \lambda=220 \mathrm{~nm}) ;$ retention times: $\mathrm{t}_{1}=$ $10.14 \min$ (major), $\mathrm{t}_{2}=11.10 \min ($ minor)<smiles>CCCCC[C@H](CCc1ccccc1)CC(=O)OC</smiles> 


\section{methyl (S)-3-phenethyloctanoate: (25d)}

Yield (66.1 mg, 84\%); colorless oil; $[\alpha]^{30}{ }_{\mathrm{D}}=-8.3\left(c\right.$ 1.1, $\left.\mathrm{CHCl}_{3}\right) ;{ }^{1} \mathrm{H} \mathrm{NMR}\left(400 \mathrm{MHz}, \mathrm{CDCl}_{3}\right) \delta$ $7.29-7.27(\mathrm{~m}, 2 \mathrm{H}), 7.18-7.15(\mathrm{~m}, 3 \mathrm{H}), 3.66(\mathrm{~s}, 3 \mathrm{H}), 2.60(\mathrm{t}, J=8.2 \mathrm{~Hz}, 2 \mathrm{H}), 2.31(\mathrm{~d}, J=6.8 \mathrm{~Hz}$, 2H), $1.96-1.90(\mathrm{~m}, 1 \mathrm{H}), 1.65-1.56(\mathrm{~m}, 2 \mathrm{H}), 1.42-1.20(\mathrm{~m}, 8 \mathrm{H}), 0.88(\mathrm{t}, J=6.8 \mathrm{~Hz}, 3 \mathrm{H}) .{ }^{13} \mathrm{C}$ NMR $\left(101 \mathrm{MHz}, \mathrm{CDCl}_{3}\right) \delta 173.9,142.5,128.4,128.3,125.7,51.5,38.9,35.9,34.9,33.7,33.0,32.1$, 26.1, 22.6, 14.1. The product was analyzed by HPLC to determine the enantiomeric excess: $97 \%$ ee (Chiralpak OD-H + OD-H, $i$-propanol/hexane $=0.8 / 99.2$, flow rate $1.0 \mathrm{~mL} / \mathrm{min}, \lambda=220 \mathrm{~nm}$ ); retention times: $\mathrm{t}_{1}=10.06 \min ($ major $), \mathrm{t}_{2}=11.12 \min ($ minor $)$.

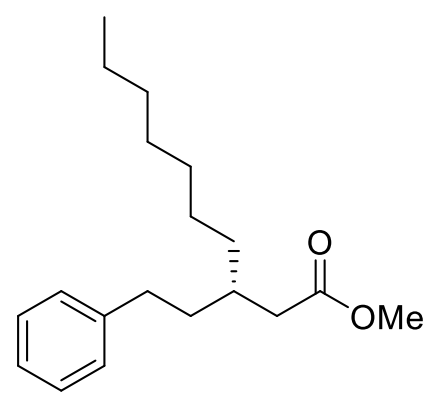

\section{methyl (S)-3-phenethyldecanoate: (25e)}

Yield (81 mg, 93\%); colorless oil; $[\alpha]^{30} \mathrm{D}=-17.3\left(c\right.$ 2.8, $\left.\mathrm{CHCl}_{3}\right) ;{ }^{1} \mathrm{H} \mathrm{NMR}\left(400 \mathrm{MHz}, \mathrm{CDCl}_{3}\right) \delta 7.29$ $-7.25(\mathrm{~m}, J=7.5 \mathrm{~Hz}, 2 \mathrm{H}), 7.21-7.14(\mathrm{~m}, 3 \mathrm{H}), 3.66(\mathrm{~s}, 3 \mathrm{H}), 2.60(\mathrm{t}, J=8.2 \mathrm{~Hz}, 2 \mathrm{H}), 2.31(\mathrm{~d}, J=$ $6.8 \mathrm{~Hz}, 2 \mathrm{H}), 1.96-1.89(\mathrm{~m}, 1 \mathrm{H}), 1.66-1.56(\mathrm{~m}, 2 \mathrm{H}), 1.44-1.19(\mathrm{~m}, 12 \mathrm{H}), 0.88(\mathrm{t}, J=6.7 \mathrm{~Hz}, 3 \mathrm{H})$. ${ }^{13} \mathrm{C}$ NMR $\left(101 \mathrm{MHz}, \mathrm{CDCl}_{3}\right) \delta 173.9,142.5,128.3,128.3,125.7,51.5,38.9,35.9,34.9,33.8,33.0$, $31.9,29.8,29.3,26.5,22.7,14.2$. The product was analyzed by HPLC to determine the enantiomeric excess: $97 \%$ ee $($ Chiralpak OD-H + OD-H, $i$-propanol/hexane $=0.8 / 99.2$, flow rate $1.0 \mathrm{~mL} / \mathrm{min}, \lambda=$ $220 \mathrm{~nm}$ ); retention $(\min ): \mathrm{t}_{1}=9.90 \min ($ major $), \mathrm{t}_{2}=11.01 \mathrm{~min}$ (minor).<smiles>COC(=O)C[C@H](C)CCc1ccccc1</smiles>

\section{methyl (S)-3-methyl-5-phenylpentanoate: (25f)}

Yield $(27.8 \mathrm{mg}, 45 \%)$; colorless oil; $[\alpha]^{30}{ }_{\mathrm{D}}=-19.0\left(c\right.$ 2.8, $\left.\mathrm{CHCl}_{3}\right) ;{ }^{1} \mathrm{H}$ NMR $\left(400 \mathrm{MHz}, \mathrm{CDCl}_{3}\right) \delta$ $7.30-7.25(\mathrm{~m}, 2 \mathrm{H}), 7.22-7.13(\mathrm{~m}, 3 \mathrm{H}), 3.66(\mathrm{~s}, 3 \mathrm{H}), 2.73-2.54(\mathrm{~m}, 2 \mathrm{H}), 2.36(\mathrm{dd}, J=14.8,6.1$ 
$\mathrm{Hz}, 1 \mathrm{H}), 2.18(\mathrm{dd}, J=14.8,8.0 \mathrm{~Hz}, 1 \mathrm{H}), 2.07-1.96(\mathrm{~m}, 1 \mathrm{H}), 1.72-1.62(\mathrm{~m}, 1 \mathrm{H}), 1.56-1.46(\mathrm{~m}$, 1H), $1.01(\mathrm{~d}, J=6.6 \mathrm{~Hz}, 3 \mathrm{H}) .{ }^{13} \mathrm{C} \mathrm{NMR}\left(101 \mathrm{MHz}, \mathrm{CDCl}_{3}\right) \delta 173.6,142.4,128.4,128.3,125.8,51.4$, $41.5,38.5,33.3,30.1,19.7$. The product was analyzed by HPLC to determine the enantiomeric excess: $91 \%$ ee (Chiralpak OD-H, $i$-propanol/hexane $=0.8 / 99.2$, flow rate $1.0 \mathrm{~mL} / \mathrm{min}, \lambda=220 \mathrm{~nm}$ ); retention times: $\mathrm{t}_{1}=6.14 \min$ (major), $\mathrm{t}_{2}=6.99 \min ($ minor) .

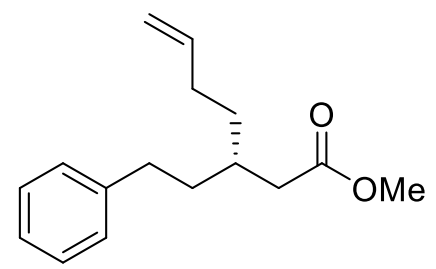

\section{methyl (S)-3-phenethylhept-6-enoate: (25g)}

Yield (69.5 mg, 94\%); colorless oil; $[\alpha]^{30} \mathrm{D}=-12.5\left(\right.$ c 1.1, $\left.\mathrm{CHCl}_{3}\right) ;{ }^{1} \mathrm{H} \mathrm{NMR}\left(400 \mathrm{MHz}, \mathrm{CDCl}_{3}\right) \delta$ $7.32-7.25(\mathrm{~m}, 2 \mathrm{H}), 7.19(\mathrm{~m}, 3 \mathrm{H}), 5.81$ (ddt, $J=16.9,10.2,6.6 \mathrm{~Hz}, 1 \mathrm{H}), 5.08-4.94(\mathrm{~m}, 2 \mathrm{H}), 3.68$ (s, $3 \mathrm{H}), 2.63(\mathrm{t}, J=8.2 \mathrm{~Hz}, 2 \mathrm{H}), 2.35(\mathrm{~d}, J=6.8 \mathrm{~Hz}, 2 \mathrm{H}), 2.15-2.04(\mathrm{~m}, 2 \mathrm{H}), 2.02-1.95(\mathrm{~m}, 1 \mathrm{H}), 1.72$ - $1.59(\mathrm{~m}, 2 \mathrm{H}), 1.48(\mathrm{~m}, 2 \mathrm{H}) .{ }^{13} \mathrm{C} \mathrm{NMR}\left(101 \mathrm{MHz}, \mathrm{CDCl}_{3}\right) \delta 173.7,142.4,138.5,128.4,128.3$, $125.8,114.7,51.5,38.7,35.7,34.4,33.0,32.9,30.8$. The product was analyzed by HPLC to determine the enantiomeric excess: $97 \%$ ee (Chiralpak OD-H + OD-H, $i$-propanol/hexane = 0.8/99.2, flow rate $1.0 \mathrm{~mL} / \mathrm{min}, \lambda=220 \mathrm{~nm}$ ); retention times: $\mathrm{t}_{1}=11.98 \mathrm{~min}$ (major), $\mathrm{t}_{2}=12.97 \mathrm{~min}$ (minor).

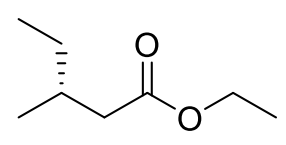

\section{ethyl (R)-3-methylpentanoate: (25h)}

Yield (41.9 mg, 97\%); colorless oil; $[\alpha]^{30} \mathrm{D}=-2.5\left(c 0.46, \mathrm{CHCl}_{3}\right) ;{ }^{1} \mathrm{H} \mathrm{NMR}\left(400 \mathrm{MHz}, \mathrm{CDCl}_{3}\right) \delta$ $4.06(\mathrm{q}, J=7.1 \mathrm{~Hz}, 2 \mathrm{H}), 2.23(\mathrm{dd}, J=14.6,6.1 \mathrm{~Hz}, 1 \mathrm{H}), 2.02(\mathrm{dd}, J=14.6,8.2 \mathrm{~Hz}, 1 \mathrm{H}), 1.86-1.75$ $(\mathrm{m}, 1 \mathrm{H}), 1.35-1.23(\mathrm{~m}, 1 \mathrm{H}), 1.21-1.10(\mathrm{~m}, 4 \mathrm{H}), 0.89-0.79(\mathrm{~m}, 6 \mathrm{H}) .{ }^{13} \mathrm{C} \mathrm{NMR}\left(101 \mathrm{MHz}, \mathrm{CDCl}_{3}\right)$ $\delta 172.4,59.1,40.5,30.9,28.3,18.2,13.3,10.3$. The product was analyzed by GC to determine the enantiomeric excess: 91\% ee (Chiraldex G-TA column, $30 \mathrm{~m}$ x $0.25 \mathrm{~mm}$, column temperature $60{ }^{\circ} \mathrm{C}$,carrier gas $\mathrm{N}_{2} 1.0 \mathrm{~mL} / \mathrm{min}$ ); retention times: $\mathrm{t}_{1}=22.75 \mathrm{~min}$ (major), $\mathrm{t}_{2}=25.43 \mathrm{~min}$ (minor). 
<smiles>CCC[C@@H](CC)CC(=O)OC</smiles>

methyl (S)-3-ethylhexanoate: (25i)

Yield $(41.8 \mathrm{mg}, 88 \%)$; colorless oil $[\alpha]^{28}{ }_{\mathrm{D}}=-5.5\left(c 0.46, \mathrm{CHCl}_{3}\right) ;{ }^{1} \mathrm{H} \mathrm{NMR}\left(400 \mathrm{MHz}, \mathrm{CDCl}_{3}\right) \delta 3.59$ (s, 3H), $2.17(\mathrm{~d}, J=6.9 \mathrm{~Hz}, 2 \mathrm{H}), 1.77-1.71(\mathrm{~m} 1 \mathrm{H}), 1.34-1.11(\mathrm{~m}, 6 \mathrm{H}), 0.84-0.78(\mathrm{~m}, 6 \mathrm{H}) .{ }^{13} \mathrm{C}$ NMR $\left(101 \mathrm{MHz}, \mathrm{CDCl}_{3}\right) \delta 173.1,50.3,37.6,35.2,34.7,25.3,18.7,13.3,9.7$. The product was analyzed by GC to determine the enantiomeric excess: 94\% ee (Chiraldex G-TA column, $30 \mathrm{~m}$ x 0.25 $\mathrm{mm}$, column temperature: $60^{\circ} \mathrm{C}$, carrier gas $\mathrm{N}_{2}, 1.0 \mathrm{~mL} / \mathrm{min}$ ); retention times: $\mathrm{t}_{1}=51.13 \mathrm{~min}$ (minor), $\mathrm{t}_{2}=52.30 \min$ (major).

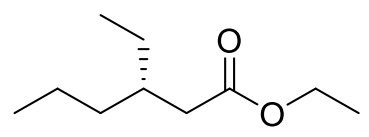

\section{ethyl (S)-3-ethylhexanoate: (25j)}

Yield (41.8 mg, 81\%); colorless oil; $[\alpha]^{30} \mathrm{D}=-5.4\left(c 0.40, \mathrm{CHCl}_{3}\right) ;{ }^{1} \mathrm{H} \mathrm{NMR}\left(400 \mathrm{MHz}, \mathrm{CDCl}_{3}\right) \delta$ $4.13(\mathrm{q}, J=7.1 \mathrm{~Hz}, 2 \mathrm{H}), 2.29-2.19(\mathrm{~m}, 2 \mathrm{H}), 1.85-1.78(\mathrm{~m}, 1 \mathrm{H}), 1.40-1.20(\mathrm{~m}, 9 \mathrm{H}), 0.91-0.85$ $(\mathrm{m}, 6 \mathrm{H}) .{ }^{13} \mathrm{C}$ NMR $\left(101 \mathrm{MHz}, \mathrm{CDCl}_{3}\right) \delta 173.8,60.1,38.9,36.2,35.7,26.3,19.7,14.3,14.3,10.8$. The product was analyzed by GC to determine the enantiomeric excess: $91 \%$ ee (Chiraldex G-TA column, $30 \mathrm{~m} \times 0.25 \mathrm{~mm}$, column temperature: $70{ }^{\circ} \mathrm{C}$, carrier gas $\mathrm{N}_{2}, 1.0 \mathrm{~mL} / \mathrm{min}$ ); retention times: $\mathrm{t}_{1}=41.97 \min$ (minor), $\mathrm{t}_{2}=43.25 \min$ (major).

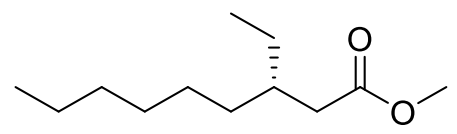

\section{methyl (S)-3-ethylnonanoate: (25k)}

Yield (51.1 mg, 85\%); colorless oil $[\alpha]^{30} \mathrm{D}=-3.8\left(c 0.50, \mathrm{CHCl}_{3}\right) ;{ }^{1} \mathrm{H} \mathrm{NMR}\left(400 \mathrm{MHz}, \mathrm{CDCl}_{3}\right) \delta$ $3.66(\mathrm{~s}, 3 \mathrm{H}), 2.24(\mathrm{~d}, J=7.0 \mathrm{~Hz}, 2 \mathrm{H}), 1.84-1.75(\mathrm{~m}, 1 \mathrm{H}), 1.42-1.20(\mathrm{~m}, 13 \mathrm{H}), 0.90-0.85(\mathrm{~m}, 6 \mathrm{H})$. ${ }^{13} \mathrm{C}$ NMR $\left(101 \mathrm{MHz}, \mathrm{CDCl}_{3}\right) \delta 174.2,51.3,38.6,36.4,33.4,31.8,29.5,26.5,26.3,22.6,14.1,10.7$. The product was analyzed by GC to determine the enantiomeric excess: 95\% ee (Chiraldex G-TA column, $30 \mathrm{~m}$ x $0.25 \mathrm{~mm}$, column temperature: $90{ }^{\circ} \mathrm{C}$, carrier gas $\mathrm{N}_{2}, 1.0 \mathrm{~mL} / \mathrm{min}$ ); retention times: $\mathrm{t}_{1}=59.90 \min$ (minor), $\mathrm{t}_{2}=61.51 \mathrm{~min}$ (major) 
<smiles>CCCCCC[C@H](CC)CC(=O)OCC</smiles>

ethyl (S)-3-ethylnonanoate: (25l)

Yield (49.5 mg, 77\%); colorless oil; $[\alpha]^{30} \mathrm{D}=-4.3\left(\right.$ c $\left.0.40, \mathrm{CHCl}_{3}\right) ;{ }^{1} \mathrm{H}$ NMR $\left(400 \mathrm{MHz}, \mathrm{CDCl}_{3}\right) \delta$ $4.13(\mathrm{q}, J=7.1 \mathrm{~Hz}, 2 \mathrm{H}), 2.22(\mathrm{~d}, J=7.0 \mathrm{~Hz}, 2 \mathrm{H}), 1.83-1.77(\mathrm{~m}, 1 \mathrm{H}), 1.41-1.19(\mathrm{~m}, 15 \mathrm{H}), 0.90-$ $0.85(\mathrm{~m}, 6 \mathrm{H}) .{ }^{13} \mathrm{C}$ NMR $\left(101 \mathrm{MHz}, \mathrm{CDCl}_{3}\right) \delta 173.7,60.1,38.9,36.4,33.4,31.8,29.6,26.5,26.3$, $22.7,14.3,14.1,10.8$. The product was analyzed by GC to determine the enantiomeric excess: $91 \%$ ee (Chiraldex G-TA column, $30 \mathrm{~m} \times 0.25 \mathrm{~mm}$, column temperature: $80{ }^{\circ} \mathrm{C}$, carrier gas $\mathrm{N}_{2}, 1.0$ $\mathrm{mL} / \mathrm{min}$ ); retention times: $\mathrm{t}_{1}=166.01 \mathrm{~min}$ (minor), $\mathrm{t}_{2}=171.37$ min (major).<smiles>CC[C@H](CC(=O)OC)C(C)C</smiles>

\section{methyl $(R)-3-e t h y l-4-m e t h y l p e n t a n o a t e: ~(25 m)$}

Yield (38 mg, 86\%); colorless oil; $[\alpha]^{30}{ }_{\mathrm{D}}=-4.2\left(c 1.20, \mathrm{CHCl}_{3}\right) ;{ }^{1} \mathrm{H} \mathrm{NMR}\left(400 \mathrm{MHz}, \mathrm{CDCl}_{3}\right) \delta 3.59$ (s, 3H), $2.21(\mathrm{dd}, J=15.1,5.9 \mathrm{~Hz}, 1 \mathrm{H}), 2.09$ (dd, $J=15.0,7.5 \mathrm{~Hz}, 1 \mathrm{H}), 1.70-1.58(\mathrm{~m}, 2 \mathrm{H}), 1.36-$ $1.25(\mathrm{~m}, 1 \mathrm{H}), 1.22-1.11(\mathrm{~m}, 1 \mathrm{H}), 0.86-0.72(\mathrm{~m}, 9 \mathrm{H}) .{ }^{13} \mathrm{C} \mathrm{NMR}\left(101 \mathrm{MHz}, \mathrm{CDCl}_{3}\right) \delta 173.7,50.4$, $41.5,34.5,28.4,22.7,18.4,17.5,10.6$. The product was analyzed by GC to determine the enantiomeric excess: $98 \%$ ee (Chiraldex G-TA column, $30 \mathrm{~m}$ x $0.25 \mathrm{~mm}$, column temperature: $60{ }^{\circ} \mathrm{C}$, carrier gas $\mathrm{N}_{2}, 1.0 \mathrm{~mL} / \mathrm{min}$ ); retention times: $\mathrm{t}_{1}=47.20 \mathrm{~min}$ (minor), $\mathrm{t}_{2}=48.81 \mathrm{~min}$ (major).<smiles>CCC(CC(=O)OC)C1CCCCC1</smiles>

\section{methyl (R)-3-cyclohexylpentanoate: (25n)}

Yield (51.7 mg, 87\%); colorless oil; $[\alpha]^{30} \mathrm{D}=-6.9\left(c\right.$ 1.1, $\left.\mathrm{CHCl}_{3}\right) ;{ }^{1} \mathrm{H} \mathrm{NMR}\left(400 \mathrm{MHz}, \mathrm{CDCl}_{3}\right) \delta 3.66$ (s, 3H), $2.32(\mathrm{dd}, J=15.1,6.2 \mathrm{~Hz}, 1 \mathrm{H}), 2.16(\mathrm{dd}, J=15.1,7.7 \mathrm{~Hz}, 1 \mathrm{H}), 1.78-1.55(\mathrm{~m}, 6 \mathrm{H}), 1.45-$ $0.93(\mathrm{~m}, 9 \mathrm{H}), 0.87(\mathrm{t}, J=7.4 \mathrm{~Hz}, 3 \mathrm{H}) .{ }^{13} \mathrm{C} \mathrm{NMR}\left(101 \mathrm{MHz}, \mathrm{CDCl}_{3}\right) \delta 174.7,51.4,42.0,40.1,35.9$, $30.0,29.1,26.8,26.7,26.7,23.8,11.7$. The product was analyzed by GC to determine the 
enantiomeric excess: 95\% ee (Chiraldex G-TA column, $30 \mathrm{~m} \mathrm{x} 0.25 \mathrm{~mm}$, column temperature: $85{ }^{\circ} \mathrm{C}$, carrier gas $\mathrm{N}_{2}, 1.0 \mathrm{~mL} / \mathrm{min}$ ); retention times: $\mathrm{t}_{1}=120.98 \mathrm{~min}$ (minor), $\mathrm{t}_{2}=124.74 \mathrm{~min}$ (major).<smiles>CCC(CC(=O)OC)c1ccccc1</smiles>

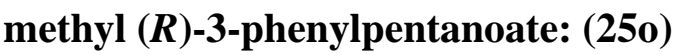

Yield (52.5 mg, 94\%); colorless oil; $[\alpha]^{30} \mathrm{D}=-12.4\left(c 0.50, \mathrm{CH}_{2} \mathrm{Cl}_{2}\right) ;{ }^{1} \mathrm{H} \mathrm{NMR}\left(400 \mathrm{MHz}, \mathrm{CDCl}_{3}\right) \delta$ $7.31-7.27(\mathrm{~m}, 2 \mathrm{H}), 7.22-7.15(\mathrm{~m}, 3 \mathrm{H}), 3.58(\mathrm{~s}, 3 \mathrm{H}), 3.04-2.97(\mathrm{~m}, 1 \mathrm{H}), 2.68-2.55(\mathrm{~m}, 2 \mathrm{H})$, $1.77-1.65(\mathrm{~m}, 1 \mathrm{H}), 1.65-1.54(\mathrm{~m}, 2 \mathrm{H}), 0.79(\mathrm{t}, J=7.3 \mathrm{~Hz}, 3 \mathrm{H}) .{ }^{13} \mathrm{C}$ NMR $\left(101 \mathrm{MHz}, \mathrm{CDCl}_{3}\right) \delta$ $172.0,142.8,127.3,126.4,125.4,50.4,42.8,40.2,28.0,10.9$. The product was analyzed by GC to determine the enantiomeric excess: $91 \%$ ee (Chiraldex G-TA column, $30 \mathrm{~m}$ x $0.25 \mathrm{~mm}$, column temperature: $100{ }^{\circ} \mathrm{C}$, carrier gas $\mathrm{N}_{2}, 1.0 \mathrm{~mL} / \mathrm{min}$ ); retention $(\mathrm{min}): \mathrm{t}_{1}=58.60 \mathrm{~min}$ (major), $\mathrm{t}_{2}=59.80$ $\min (\operatorname{minor})$.

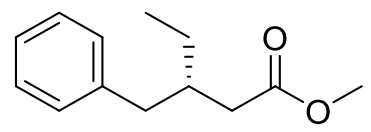

\section{methyl $(S)$-3-benzylpentanoate: (25p)}

Yield (59.4 mg, 96\%); colorless oil; $[\alpha]^{30} \mathrm{D}=+7.5\left(c\right.$ 1.2, $\left.\mathrm{CH}_{2} \mathrm{Cl}_{2}\right) ;{ }^{1} \mathrm{H}$ NMR $\left(400 \mathrm{MHz}, \mathrm{CDCl}_{3}\right) \delta$ $7.31-7.24(\mathrm{~m}, 2 \mathrm{H}), 7.22-7.14(\mathrm{~m}, 3 \mathrm{H}), 3.62(\mathrm{~s}, 3 \mathrm{H}), 2.66(\mathrm{dd}, J=13.6,6.7 \mathrm{~Hz}, 1 \mathrm{H}), 2.53(\mathrm{dd}, J=$ 13.6, $7.4 \mathrm{~Hz}, 1 \mathrm{H}), 2.30-2.19(\mathrm{~m}, 2 \mathrm{H}), 2.12(\mathrm{dt}, J=13.5,6.9 \mathrm{~Hz}, 1 \mathrm{H}), 1.44-1.23(\mathrm{~m}, 2 \mathrm{H}), 0.92(\mathrm{t}, J$ $=7.5 \mathrm{~Hz}, 3 \mathrm{H}) .{ }^{13} \mathrm{C} \mathrm{NMR}\left(101 \mathrm{MHz}, \mathrm{CDCl}_{3}\right) \delta 173.8,140.3,129.3,128.2,126.0,77.2,51.4,40.0$, 38.5, 37.9, 26.2, 11.0. The product was analyzed by HPLC to determine the enantiomeric excess: 92\% ee (Chiralpak OD-H, $i$-propanol/hexane $=0.8 / 99.2$, flow rate $1.0 \mathrm{~mL} / \mathrm{min}, \lambda=220 \mathrm{~nm}$ ); retention times: $\mathrm{t}_{1}=5.52 \min$ (major), $\mathrm{t}_{2}=6.65 \min$ (minor).<smiles>CC[C@@H](CC(=O)OC)c1ccco1</smiles>

methyl (R)-3-(furan-2-yl)pentanoate: (25q) 
Yield (40.5 mg, 74\%); colorless oil; $[\alpha]^{30} \mathrm{D}=-2.3\left(\right.$ c 1.7, $\left.\mathrm{CH}_{2} \mathrm{Cl}_{2}\right) ;{ }^{1} \mathrm{H}$ NMR $\left(400 \mathrm{MHz}, \mathrm{CDCl}_{3}\right) \delta$ $7.31(\mathrm{dd}, J=1.9,0.9 \mathrm{~Hz}, 1 \mathrm{H}), 6.27(\mathrm{dd}, J=3.1,1.9 \mathrm{~Hz}, 1 \mathrm{H}), 6.03(\mathrm{~d}, J=3.1 \mathrm{~Hz}, 1 \mathrm{H}), 3.64(\mathrm{~s}, 3 \mathrm{H})$, $3.17(\mathrm{~m}, 1 \mathrm{H}), 2.68-2.54(\mathrm{~m}, 2 \mathrm{H}), 1.74-1.63(\mathrm{~m}, 2 \mathrm{H}), 0.85(\mathrm{t}, J=7.4 \mathrm{~Hz}, 3 \mathrm{H}) .{ }^{13} \mathrm{C} \mathrm{NMR}(101 \mathrm{MHz}$, $\left.\mathrm{CDCl}_{3}\right) \delta 172.7,157.0,141.1,109.9,105.2,51.6,38.5,37.0,26.7,11.5$. The product was analyzed by GC to determine the enantiomeric excess: $97 \%$ ee (Chiraldex G-TA column, $30 \mathrm{~m}$ x $0.25 \mathrm{~mm}$, column temperature: $90{ }^{\circ} \mathrm{C}$, carrier gas $\mathrm{N}_{2}, 1.0 \mathrm{~mL} / \mathrm{min}$ ); retention times $(\mathrm{min})$ : $\mathrm{t}_{1}=37.51 \mathrm{~min}$ (major), $\mathrm{t}_{2}=38.91 \mathrm{~min}$ (minor).

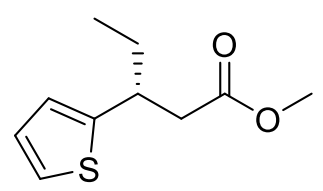

\section{methyl $(R)-3$-(thiophen-2-yl)pentanoate: (25r)}

Yield (50 mg, 84\%); colorless oil; $[\alpha]^{30} \mathrm{D}=-25.2\left(c\right.$ 2.6, $\left.\mathrm{CH}_{2} \mathrm{Cl}_{2}\right) ;{ }^{1} \mathrm{H}$ NMR (400 MHz, $\left.\mathrm{CDCl}_{3}\right) \delta 7.14$ $(\mathrm{d}, J=5.0 \mathrm{~Hz}, 1 \mathrm{H}), 6.91(\mathrm{dd}, J=5.0,3.4 \mathrm{~Hz}, 1 \mathrm{H}), 6.82(\mathrm{~d}, J=3.4 \mathrm{~Hz}, 1 \mathrm{H}), 3.63(\mathrm{~s}, 3 \mathrm{H}), 3.36(\mathrm{~m}$, $1 \mathrm{H}), 2.71-2.57(\mathrm{~m}, 2 \mathrm{H}), 1.81-1.70(\mathrm{~m}, 1 \mathrm{H}), 1.70-1.57(\mathrm{~m}, 1 \mathrm{H}), 0.87(\mathrm{t}, J=7.4 \mathrm{~Hz}, 3 \mathrm{H}) .{ }^{13} \mathrm{C}$ NMR $\left(101 \mathrm{MHz}, \mathrm{CDCl}_{3}\right) \delta 172.6,147.7,126.5,124.0,123.1,51.6,42.2,39.2,30.1,11.8$. The product was analyzed by GC to determine the enantiomeric excess: 96\% ee (Chiraldex G-TA column, $30 \mathrm{~m}$ x $0.25 \mathrm{~mm}$, column temperature: $100{ }^{\circ} \mathrm{C}$, carrier gas $\mathrm{N}_{2}, 1.0 \mathrm{~mL} / \mathrm{min}$ ); retention times (min): $\mathrm{t}_{1}$ $=64.46 \min$ (major), $\mathrm{t}_{2}=66.52 \mathrm{~min}$ (minor). 


\section{References}

(1) Hayashi, T.; Mise, T.; Fukushima, M.; Kagotani, M.; Nagashima, M.; Hamada, Y.; Matsumoto, A.; Kawakami, S.; Konishi, M.; Yamamoto, K.; Kumata, M. Asymmetric Synthesis Catalyzed by Chiral Ferrocenylphosphine-Transition Metal Complexes. I. Preparation of Chiral Ferrocenylphosphines. Bull. Chem. Soc. Jpn. 1980, 53, 1138-1151.

(2) Togni, A.; Brentel, C.; Landert, H.; Tijani, A. A Novel Easily Accessible Chiral Ferrocenyldiphosphine for Highly Enantioselective Hydrogenation, Allylic Alkylation, and Hydroboration Reactions. J. Am. Chem. Soc. 1994, 116, 4062-4066.

(3) Wang, C.-J.; Liang, G.; Xue, Z.-Y.; Gao, F. Highly Enantioselective 1,3-Dipolar Cycloaddition of Azomethine Ylides Catalyzed by Copper(I)/TF-BiphamPhos Complexes. J. Am. Chem. Soc. 2008, $130,17250-17251$.

(4) Liu, J.; Cao, C.-G.; Sun, H.-B.; Zhang, X.; Niu, D. Catalytic Asymmetric Umpolung Allylation of Imines. J. Am. Chem. Soc. 2016, 138, 13103-13106.

(5) Wei, L.; Zhu, Q.; Xiao, L.; Tao, H.-Y.; Wang, C.-J. Synergistic catalysis for cascade allylation and 2-aza-cope rearrangement of azomethine ylides. Nat. Commun. 2019, 10, 1594.

(6) Chang, X.; Sun, X.-S.; Che, C.; Hu, Y.-Z.; Tao, H.-Y.; Wang, C.-J. Copper(I)-Catalyzed Kinetic Resolution of exo-3-Oxodicyclopentadienes and endo-3-Oxodicyclopentadiene. Org. Lett. 2019, 21, 1191-1196.

(7) López, F.; Harutyunyan, S. R.; Minnaard, A. J.; Feringa, B. L. Copper-Catalyzed Enantioselective Conjugate Addition of Grignard Reagents to $\alpha, \beta$-Unsaturated Esters. Angew. Chem., Int. Ed. 2005, $44,2752-2756$.

(8) Wang, S.-Y.; Ji S.-J.; Loh T.-P. Cu(I) Tol-BINAP-Catalyzed Enantioselective Michael Reactions of Grignard Reagents and Unsaturated Esters. J. Am. Chem. Soc. 2007, 129, 276-277. 
11. NMR, HPLC and GC Spectra

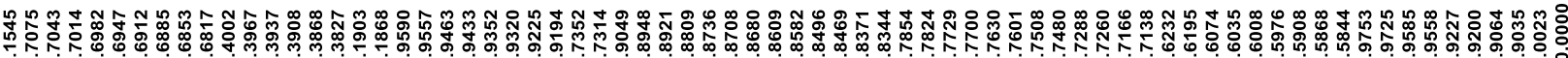

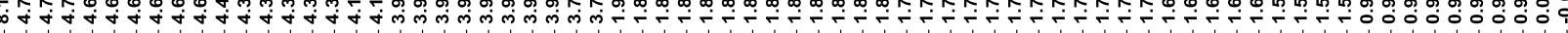

$\overbrace{\mathrm{Fe}}^{\mathrm{Bu}} \mathrm{CO}_{2} \mathrm{Me}$

(8)

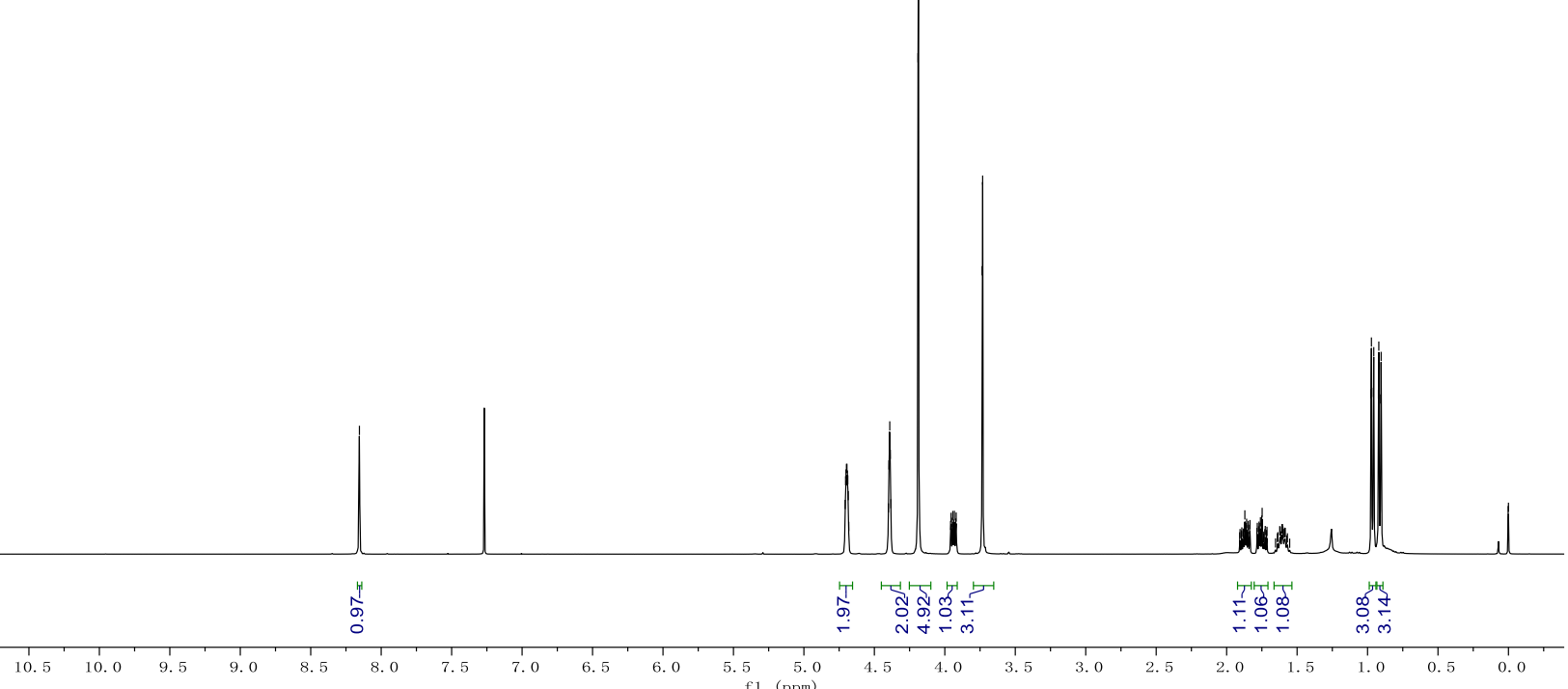

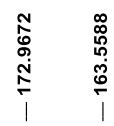

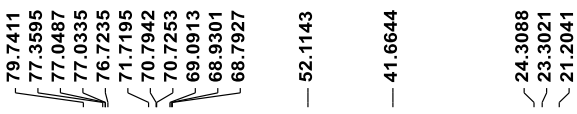

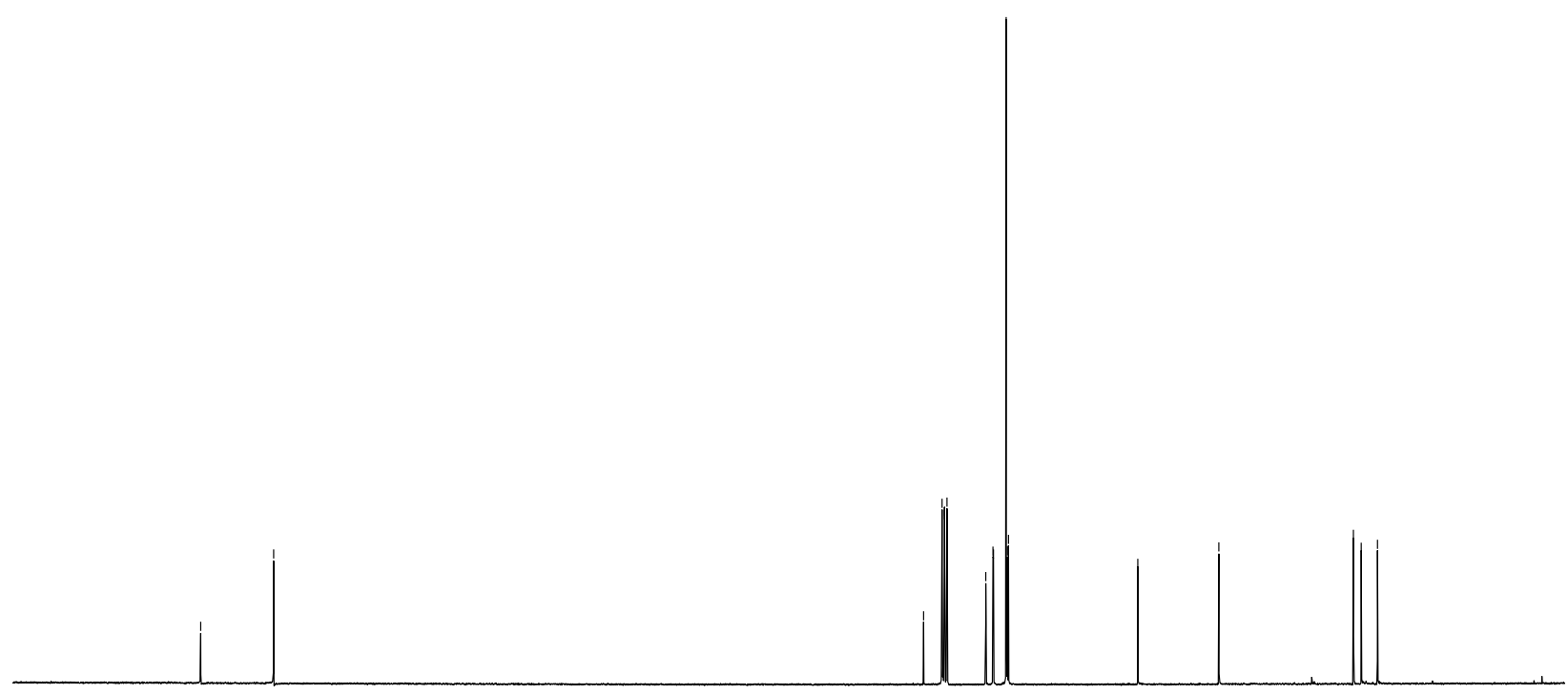

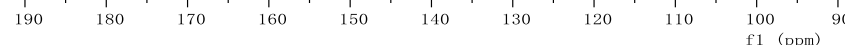



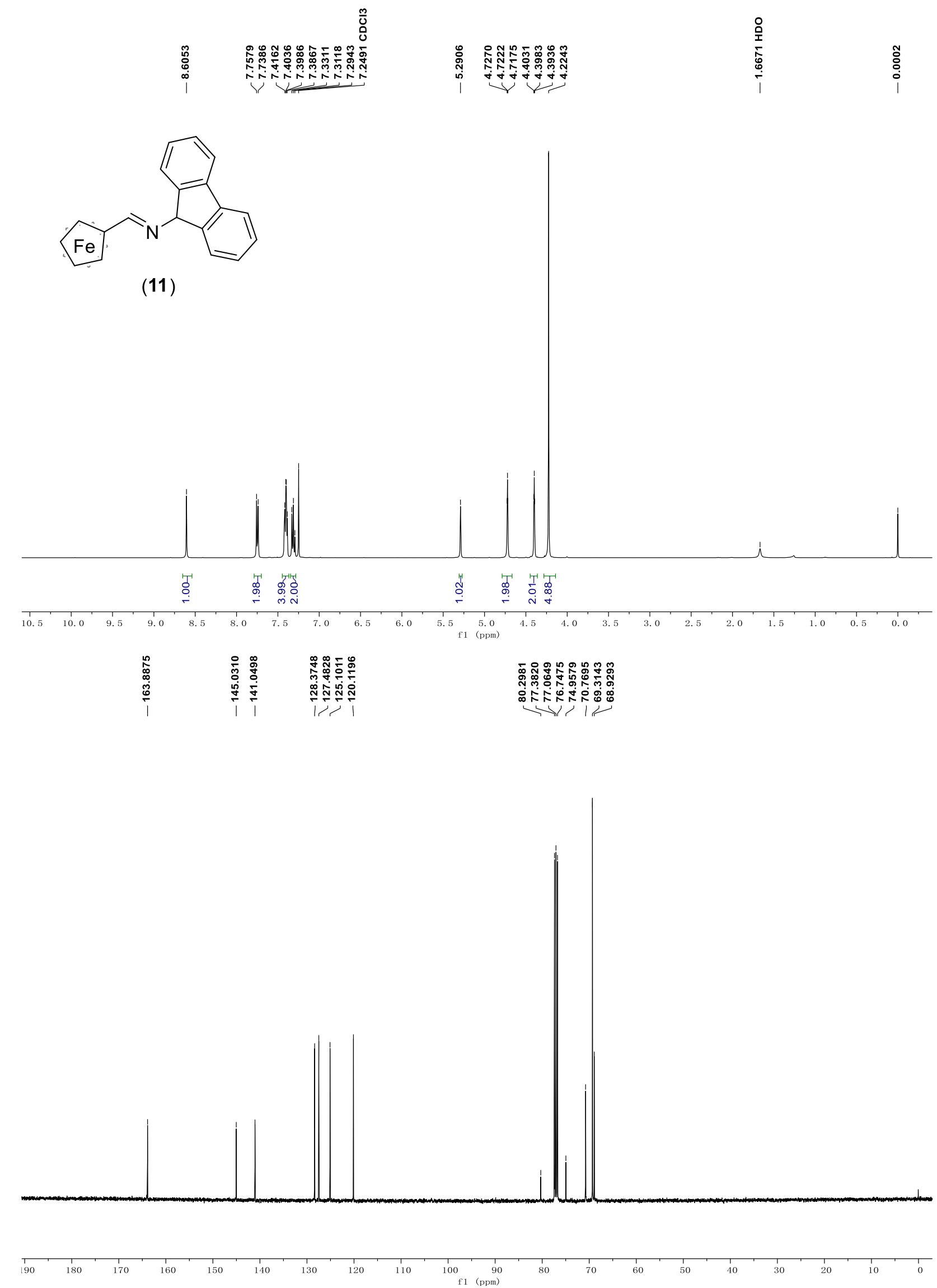

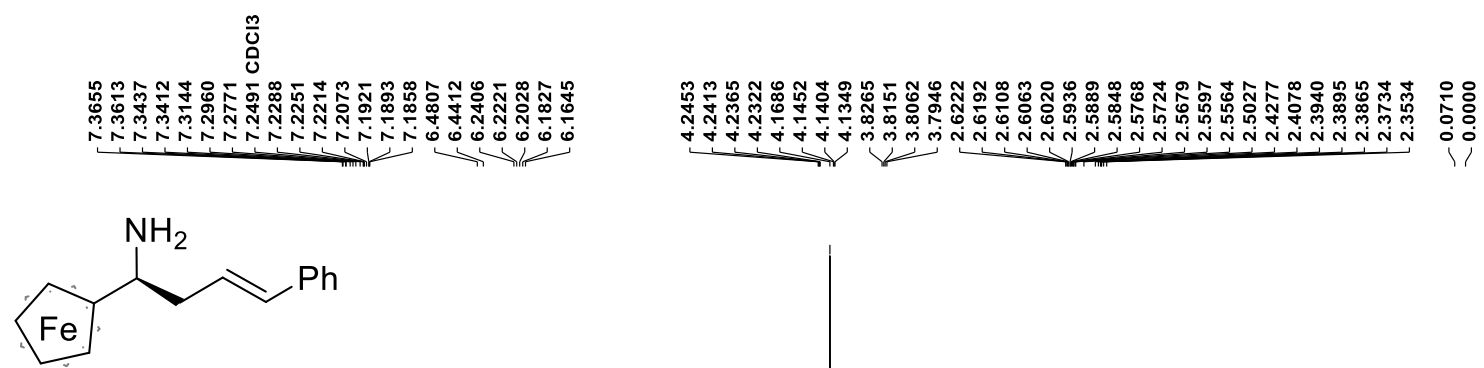

(15a)

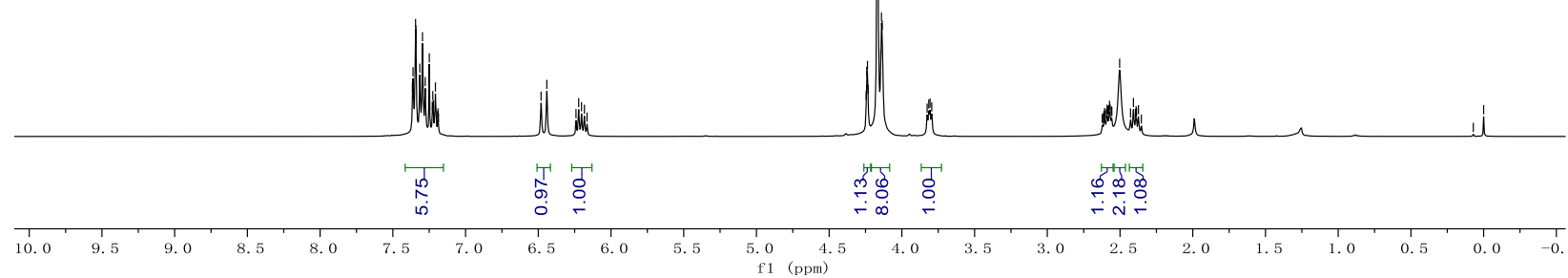

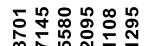

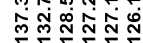

14

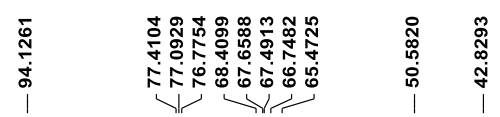

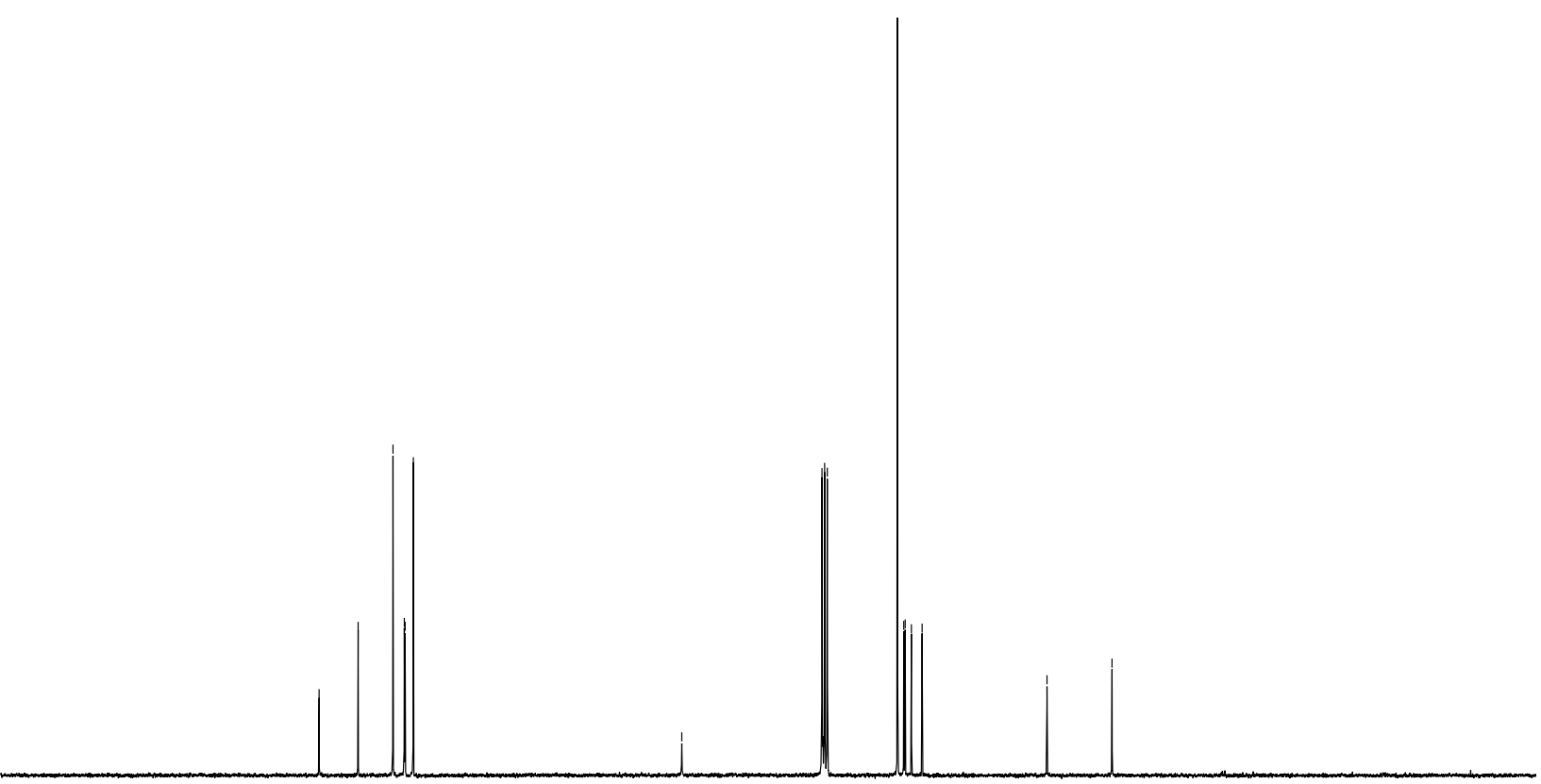

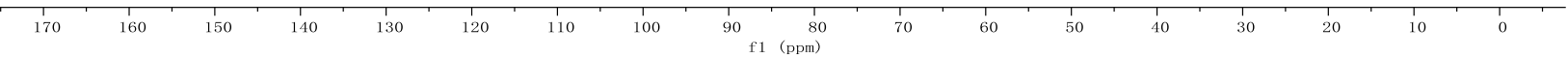



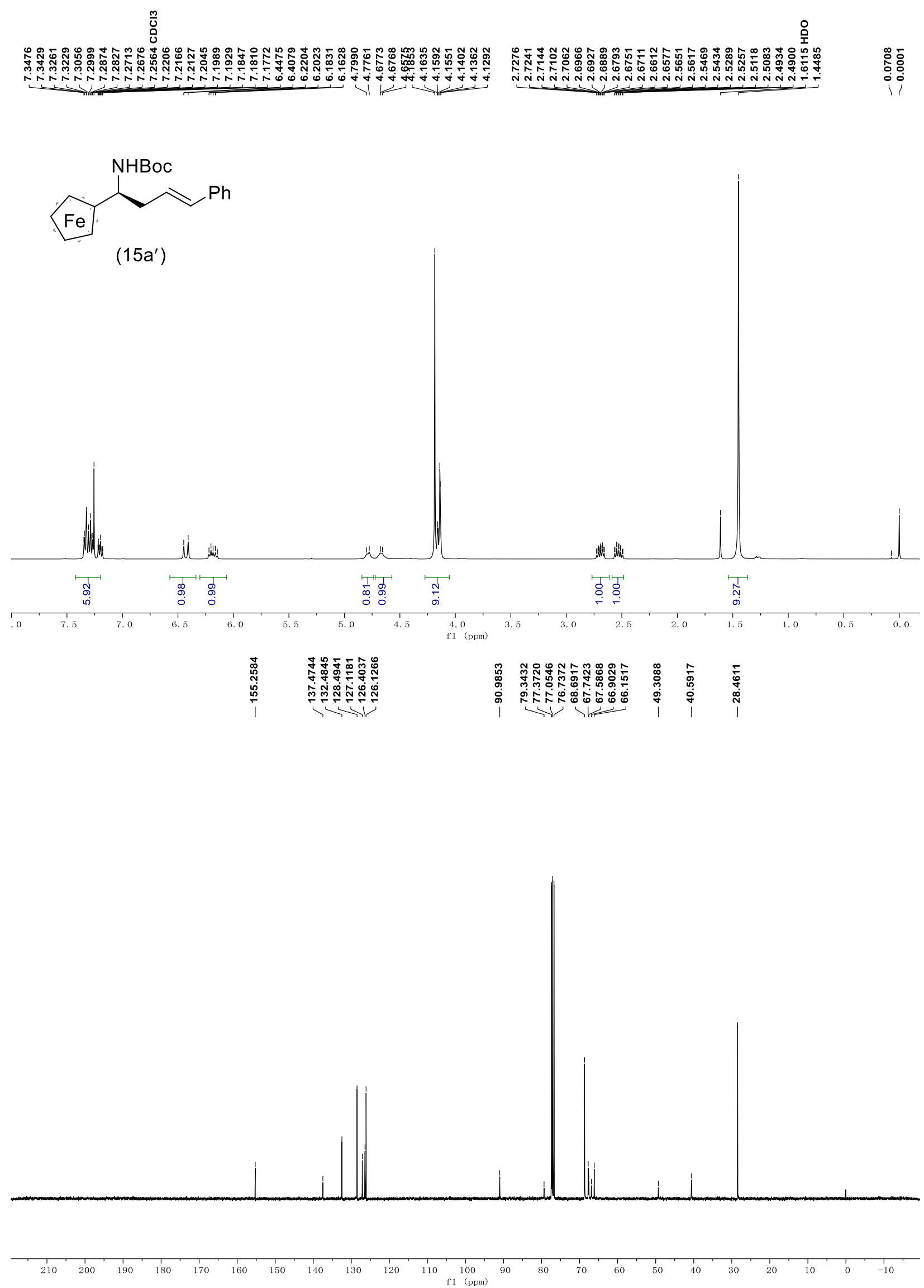


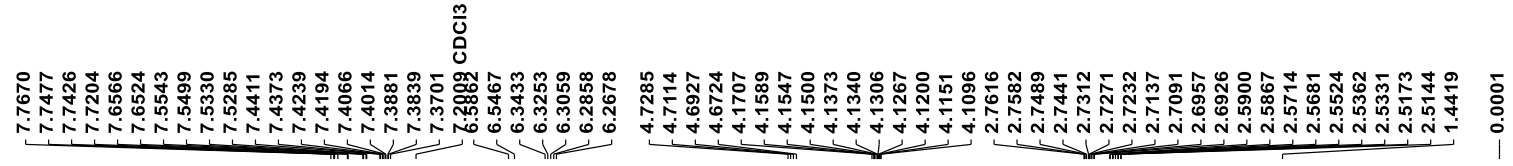<smiles>CC(C)(C)OC(=O)CC=Cc1ccc2ccccc2c1</smiles>

$\left(15 b^{\prime}\right)$
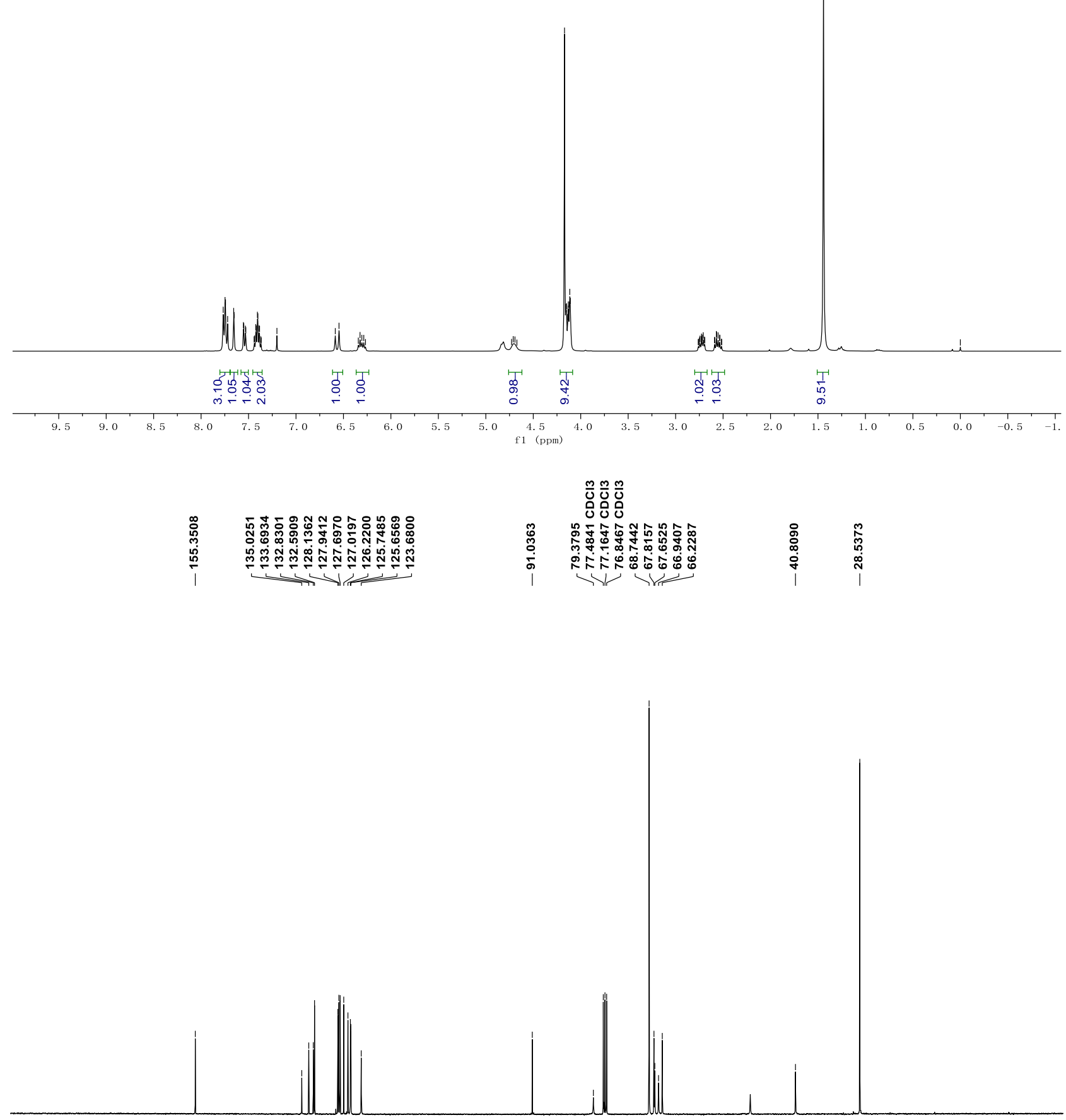

90

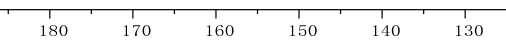

120

$110 \quad 100$

1 190 
怘

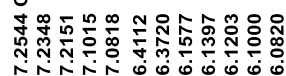

$\rightarrow 12$

0100 일

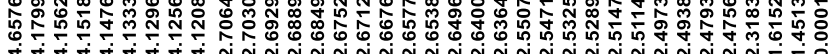

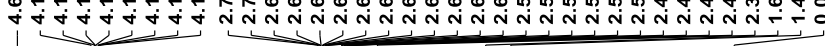<smiles>Cc1ccc(/C=C/CC(N)C2CCCC2)cc1</smiles>

$\left(15 c^{\prime}\right)$

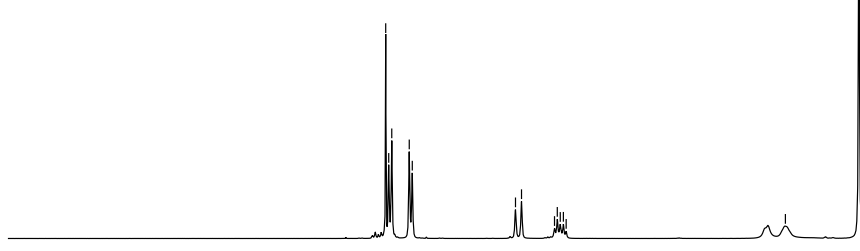

惨

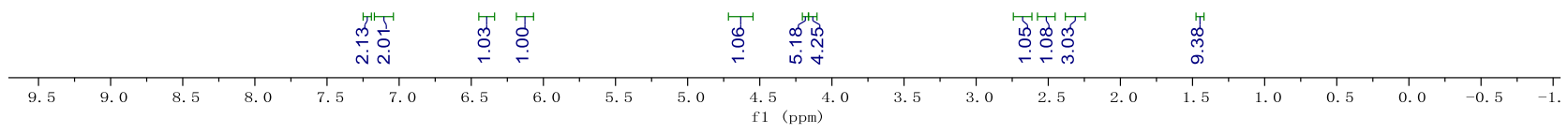

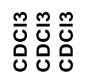

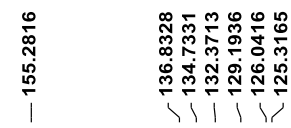

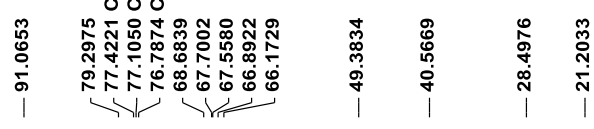

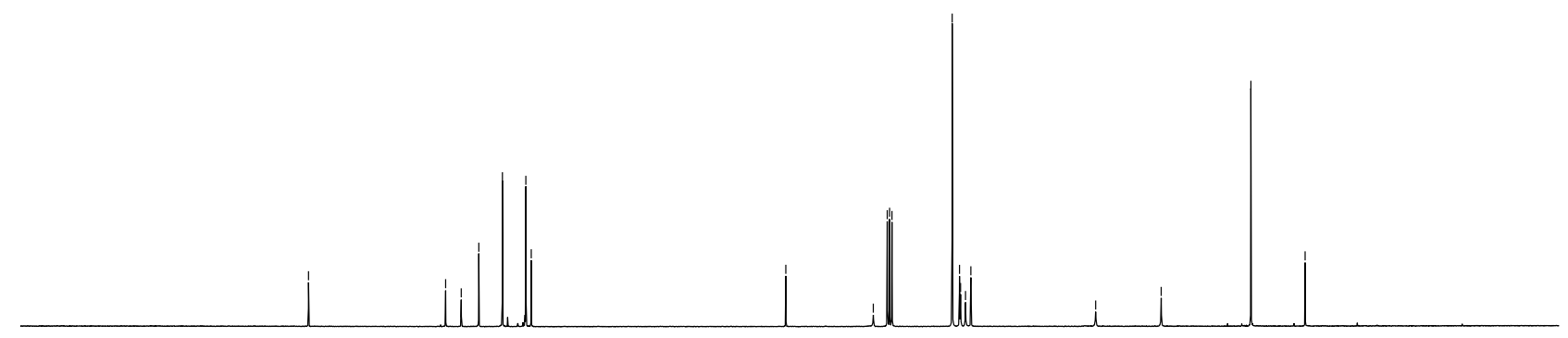

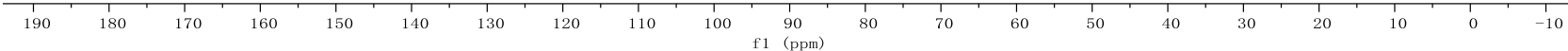



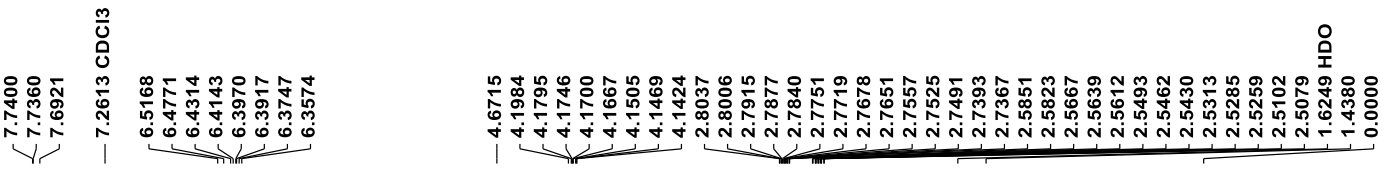<smiles>O=C(NC(C/C=C/c1cc(C(F)(F)F)cc(C(F)(F)F)c1)C1CCCC1)C(F)(F)F</smiles>

$\left(15 d^{\prime}\right)$
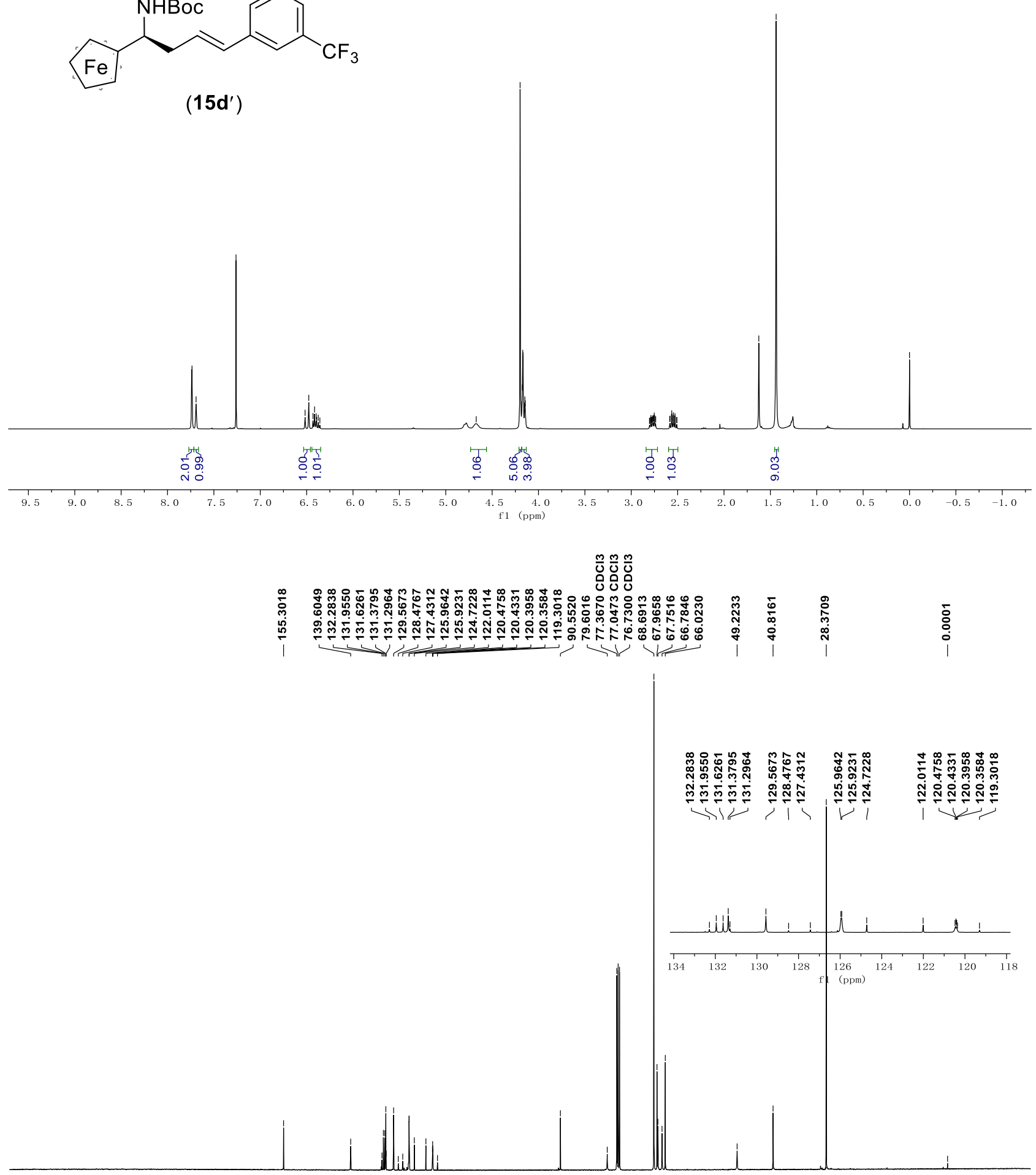

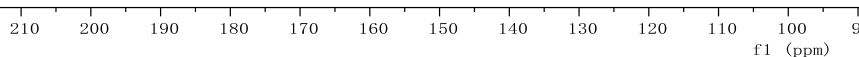




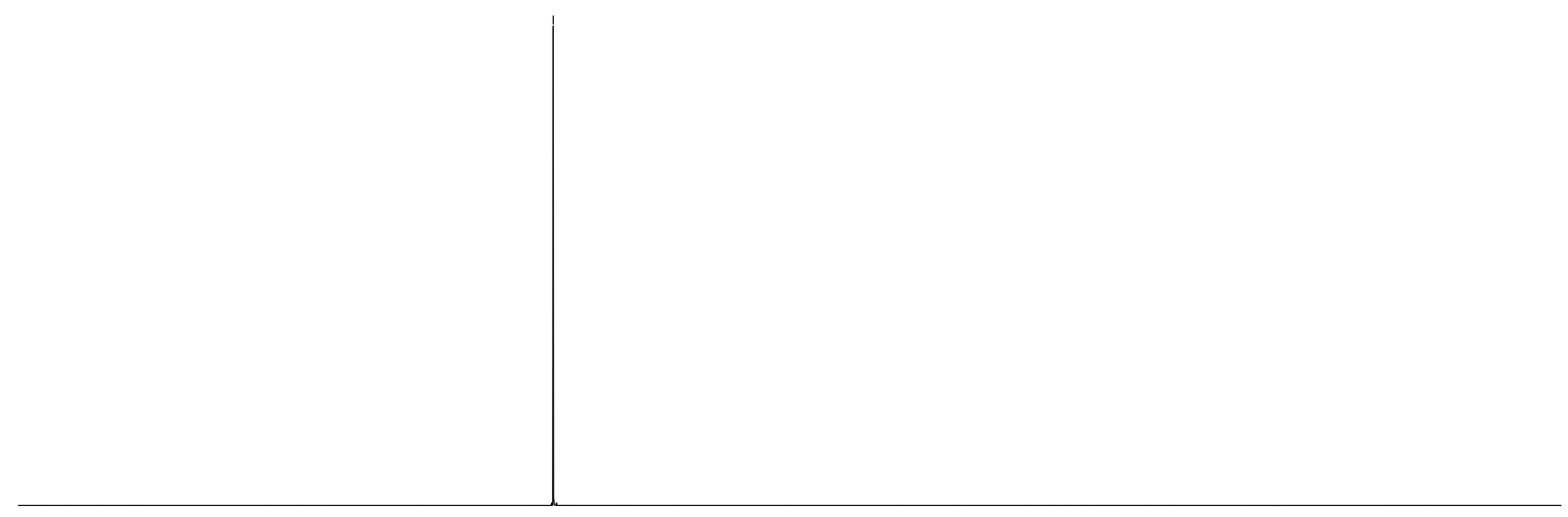

$\underset{20}{10} \quad$\begin{tabular}{lllllllllllllllllllllllllllllllllllll}
1 & 10 & 0 & -10 & -20 & -30 & -40 & -50 & -60 & -70 & -80 & -90 & -100 & -110 & -120 & -130 & -140 & -150 & -160 & -170 & -180 & -190 & -200 & -210 & -2 \\
\hline
\end{tabular} 
\%ั0

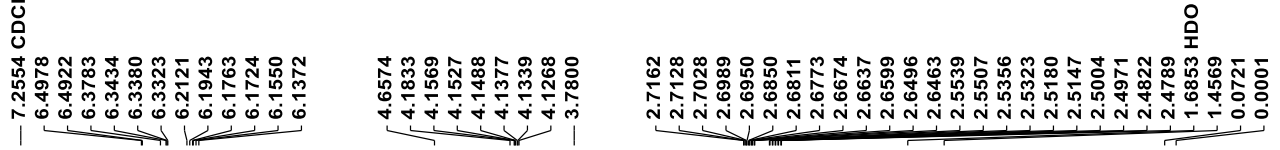

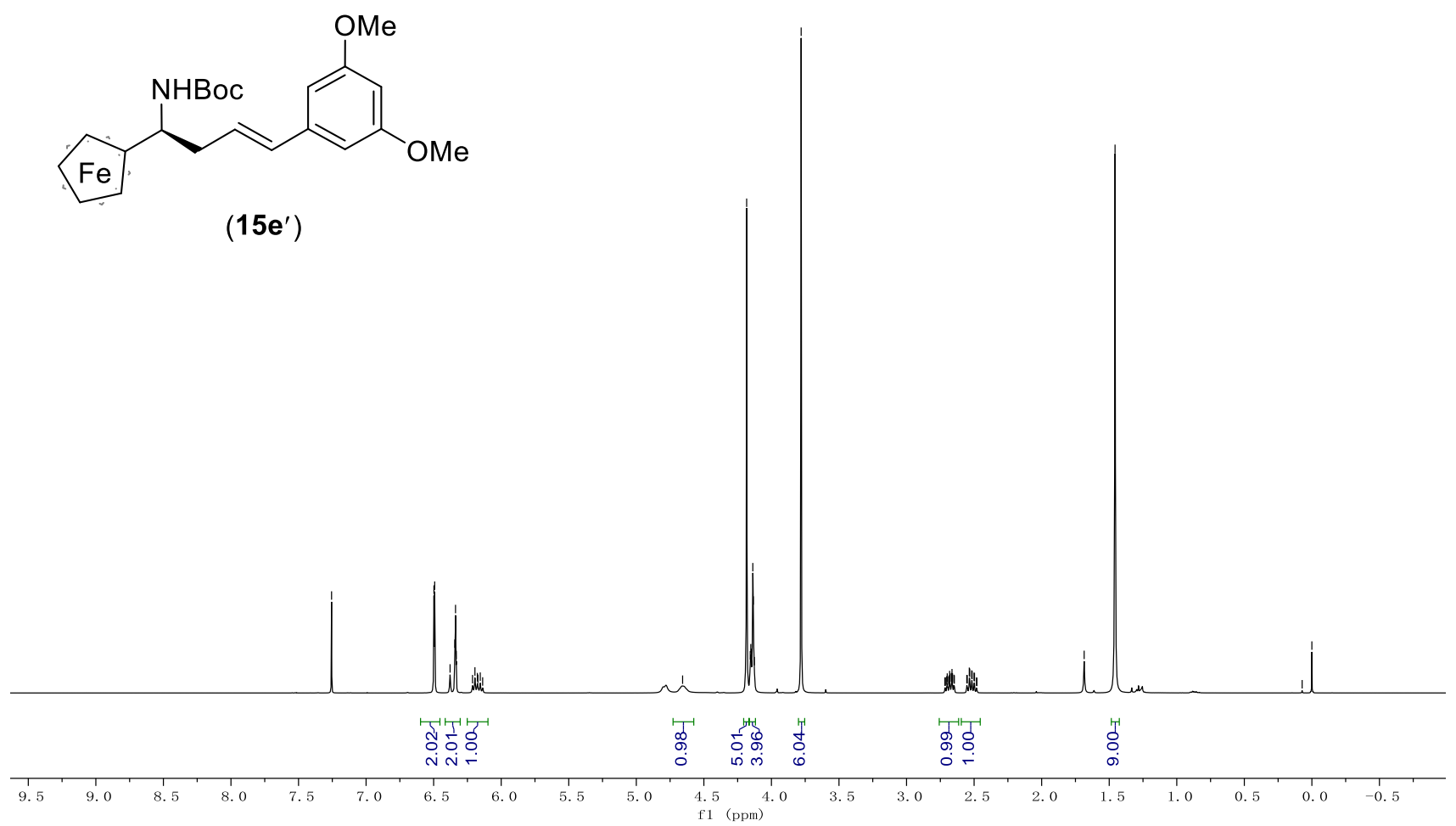

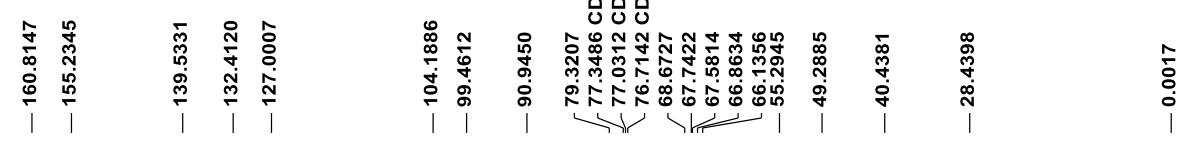
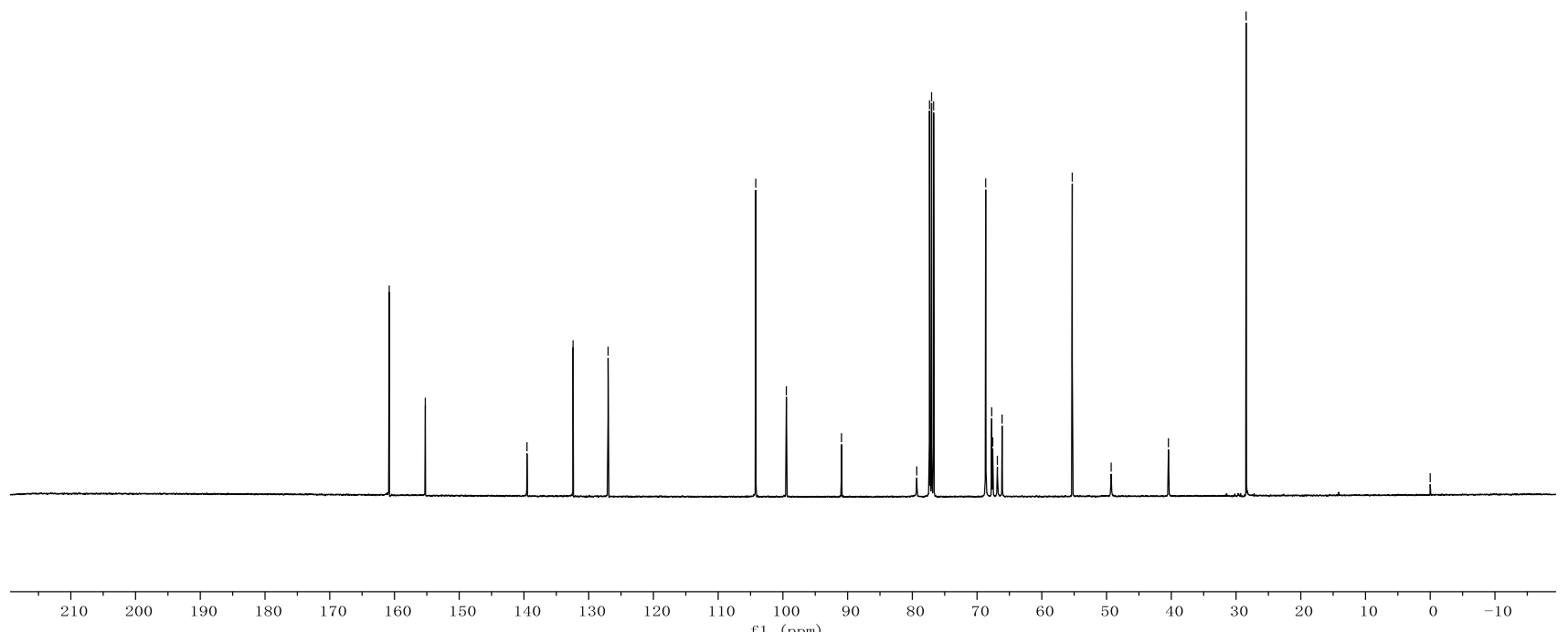


\section{㠃

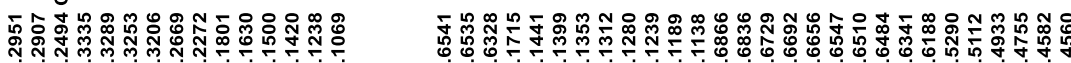

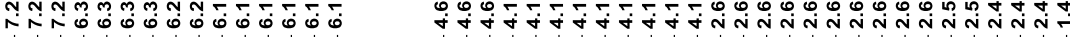 \\ $\overline{\grave{o}}$}

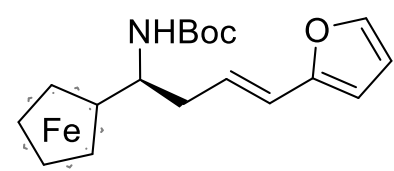

$\left(15 f^{\prime}\right)$

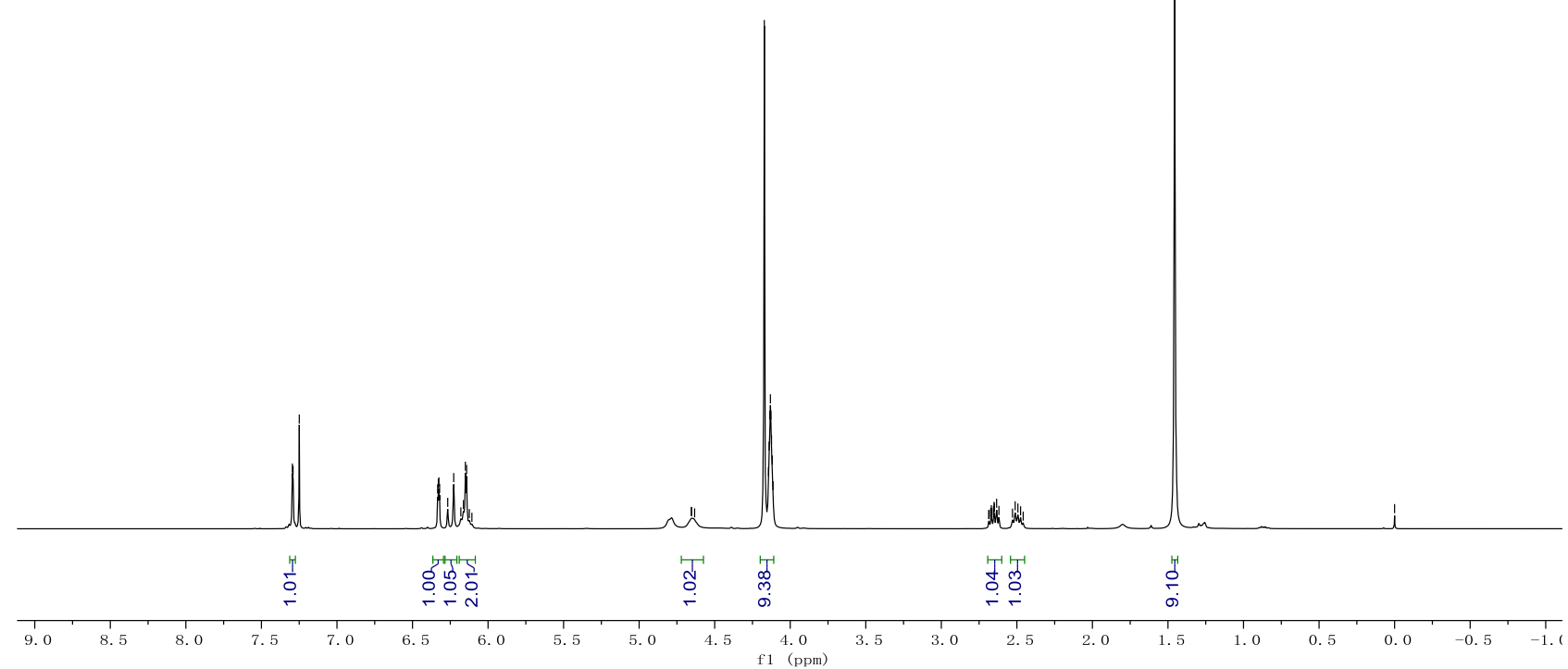

㧝 |
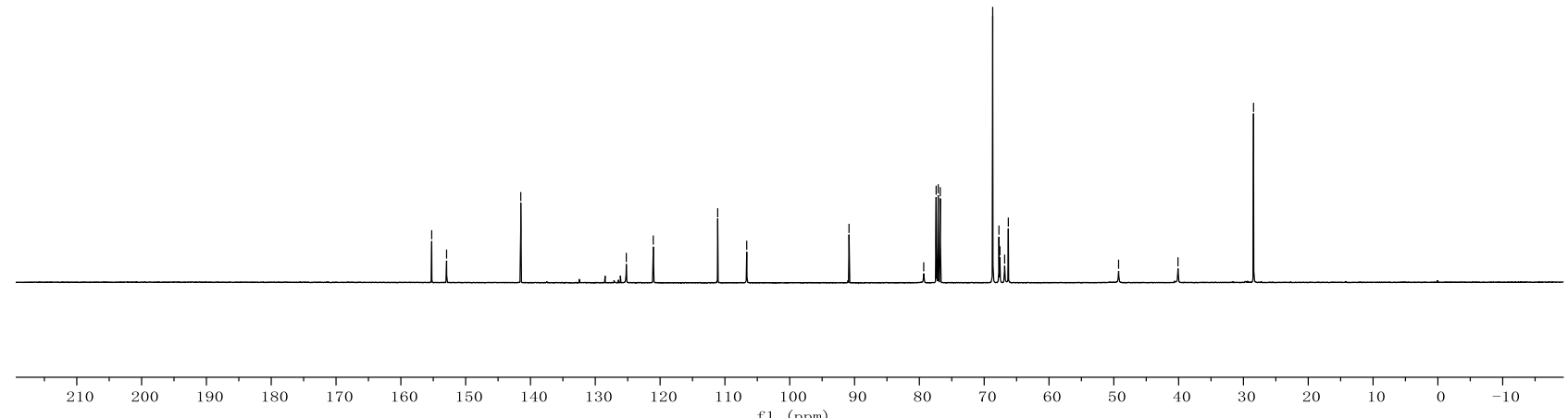

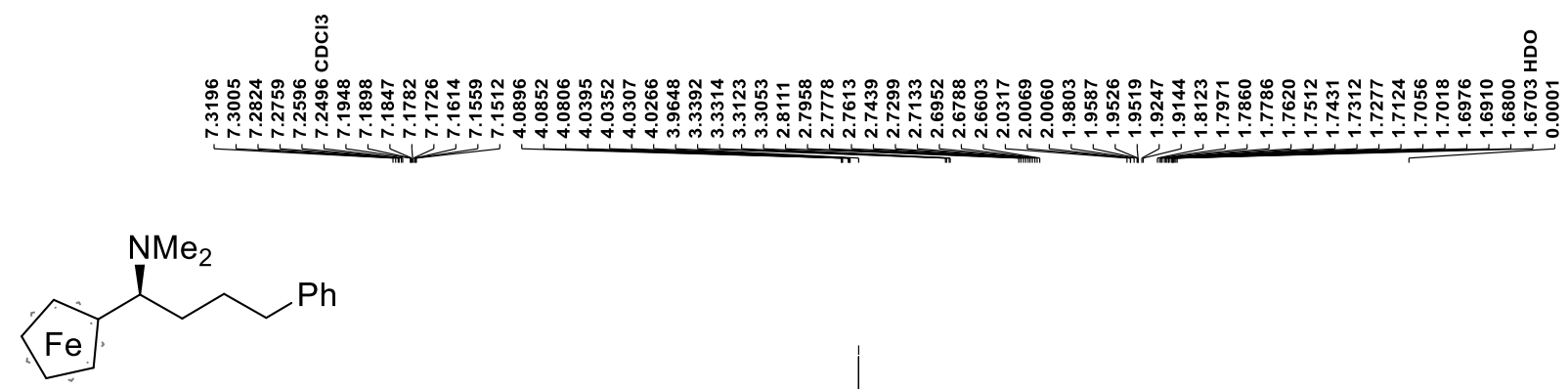

(16)
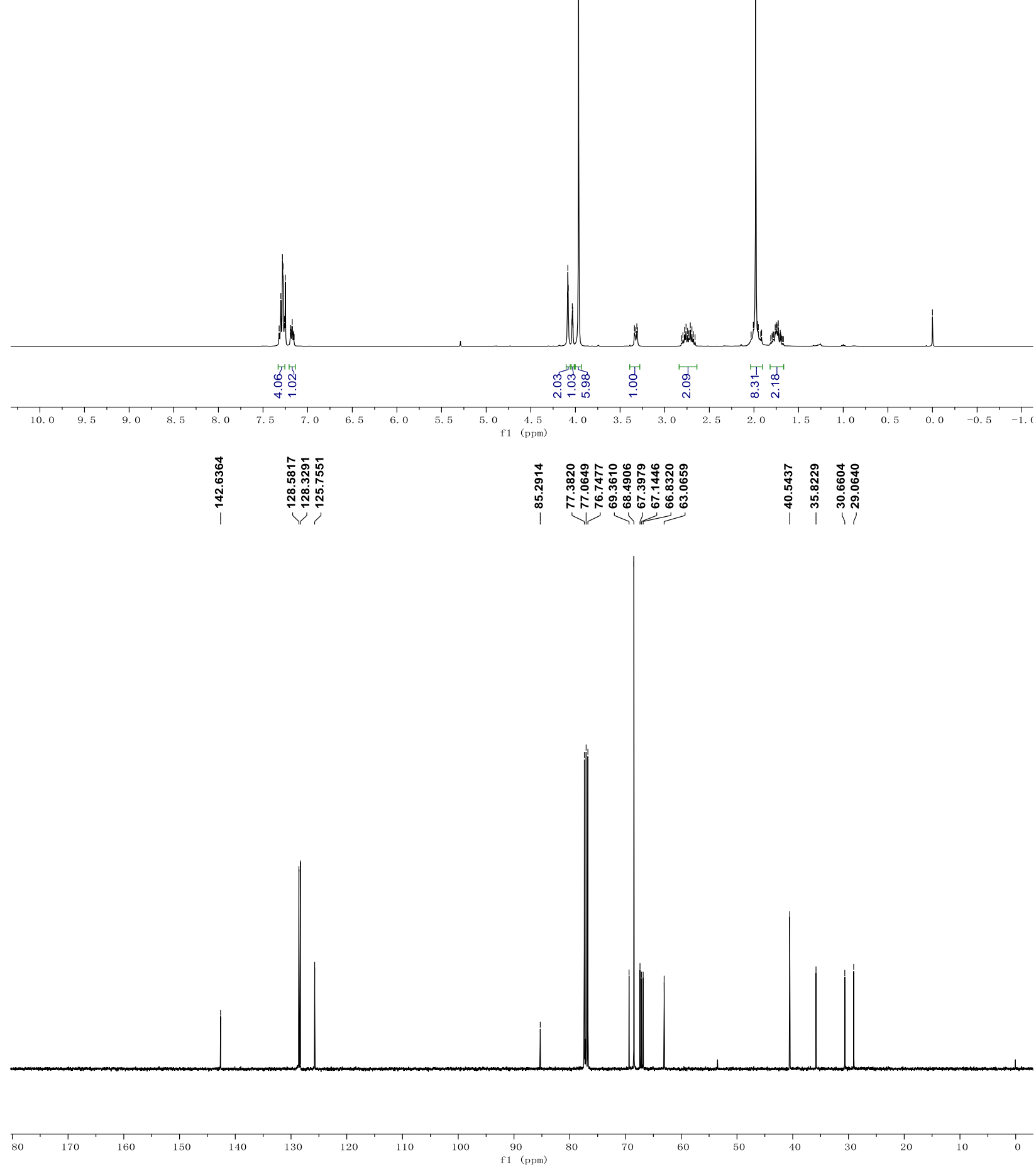


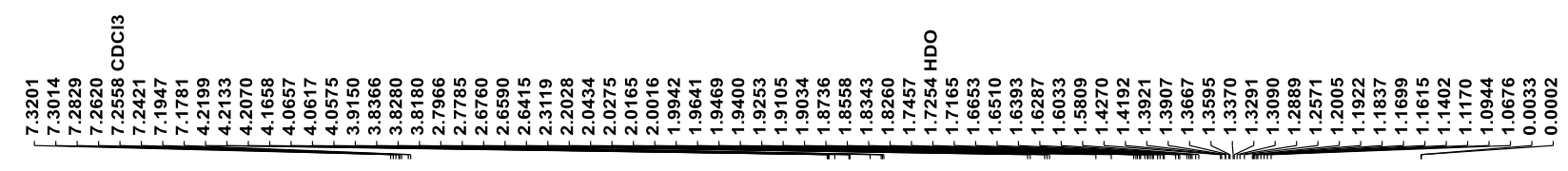

$\mathrm{Fe}_{\mathrm{Fe}}^{\mathrm{PCh}} \mathrm{NM}_{3}$

$(S, R p)-17 \mathbf{a}$
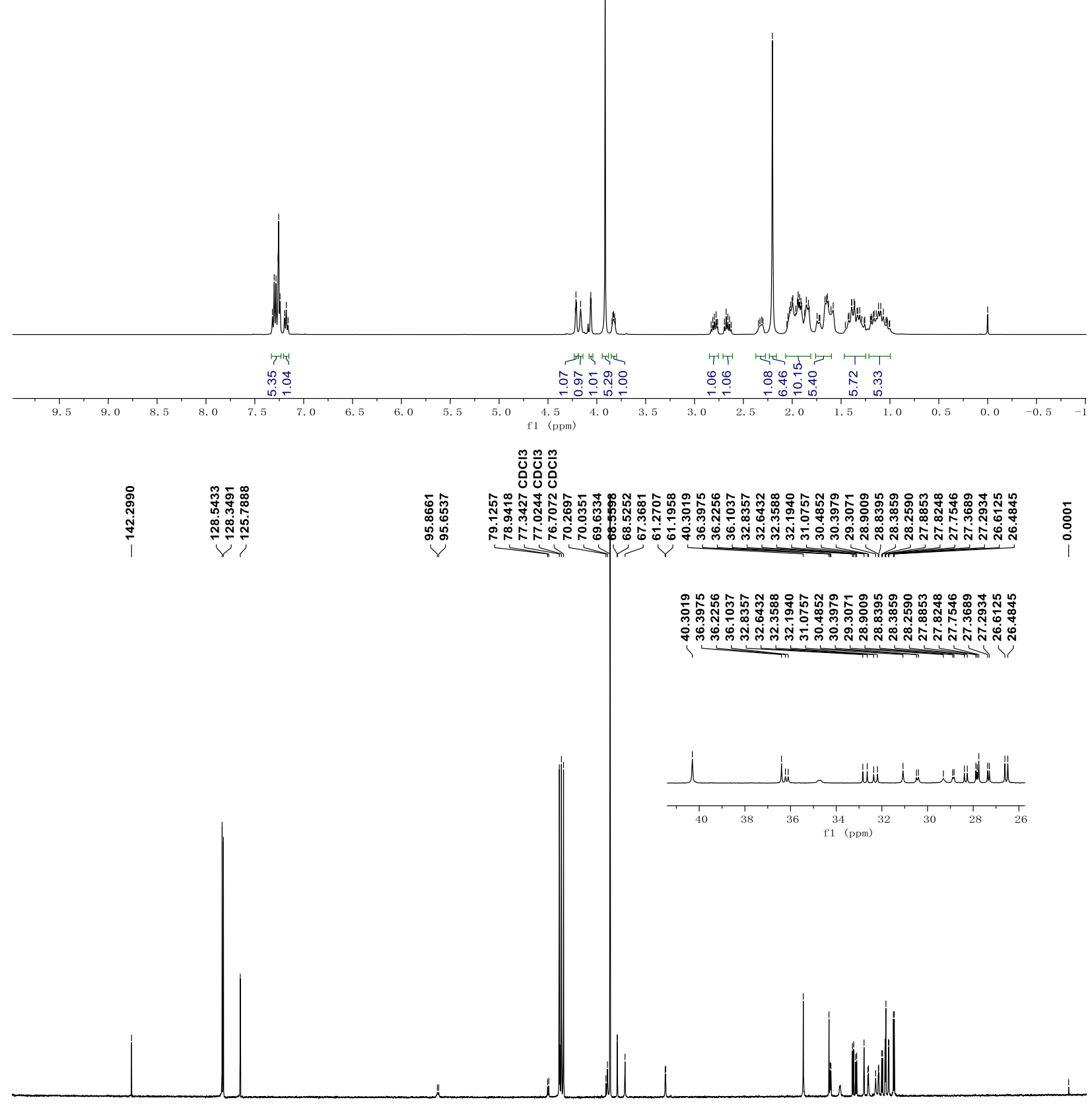


\section{ָั๊}

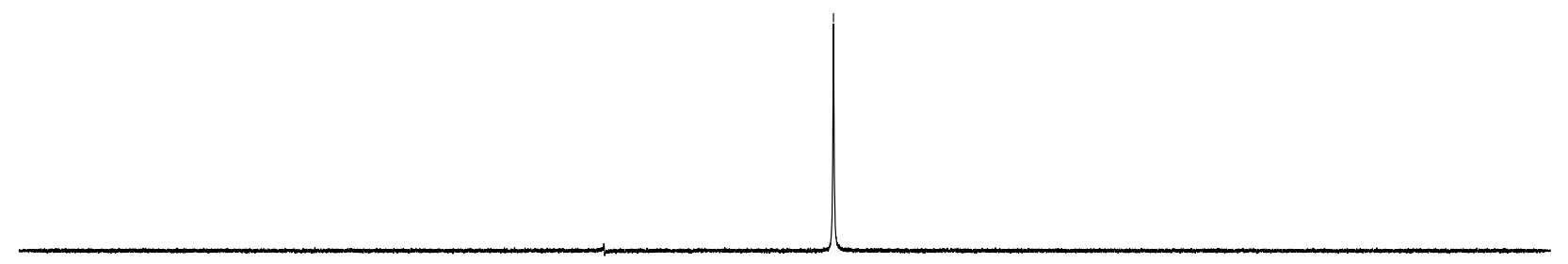

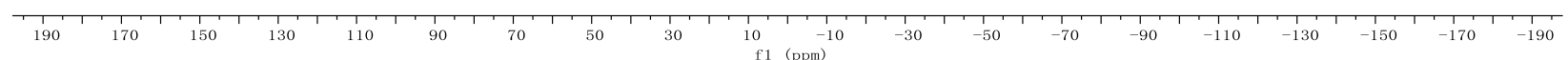

ั0

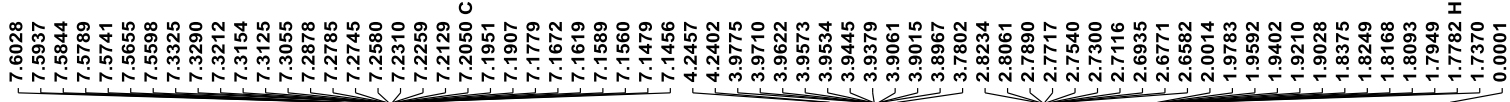

Fe

$(S, R p)-17 b$

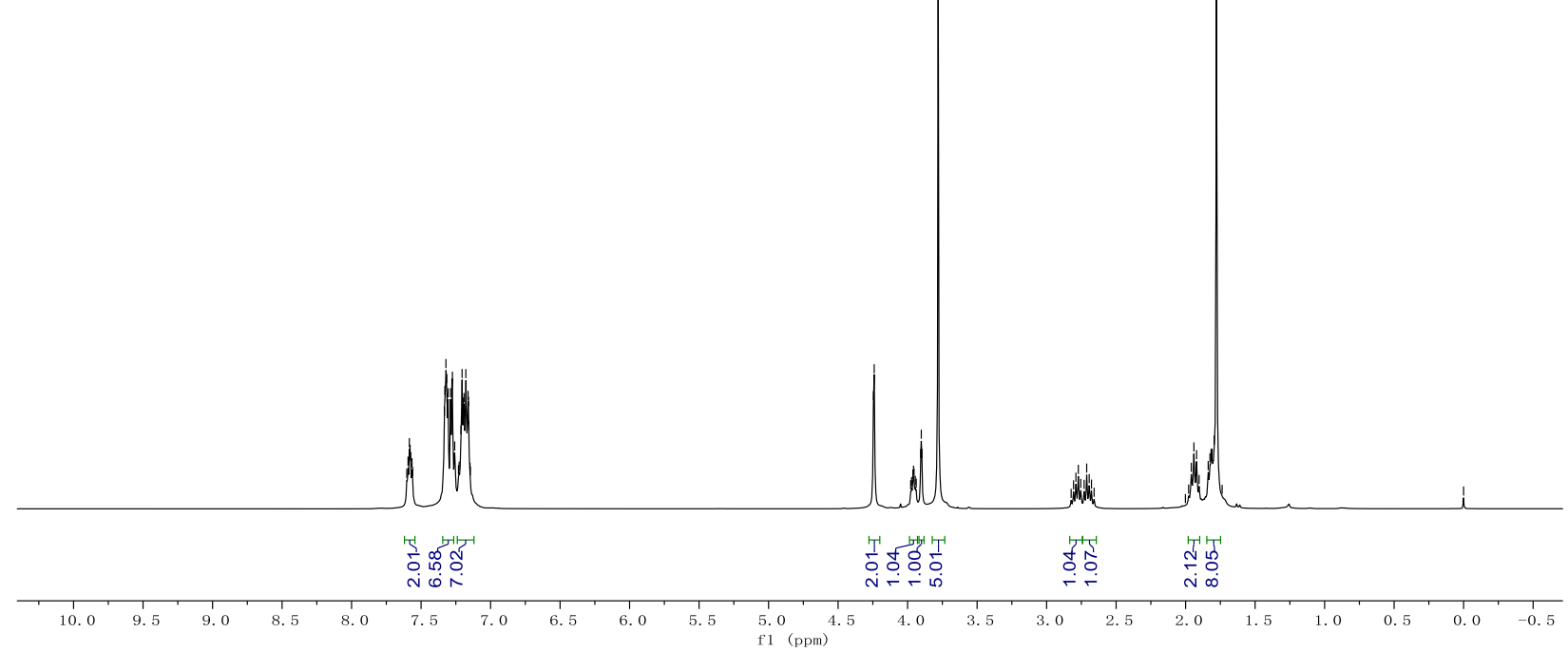



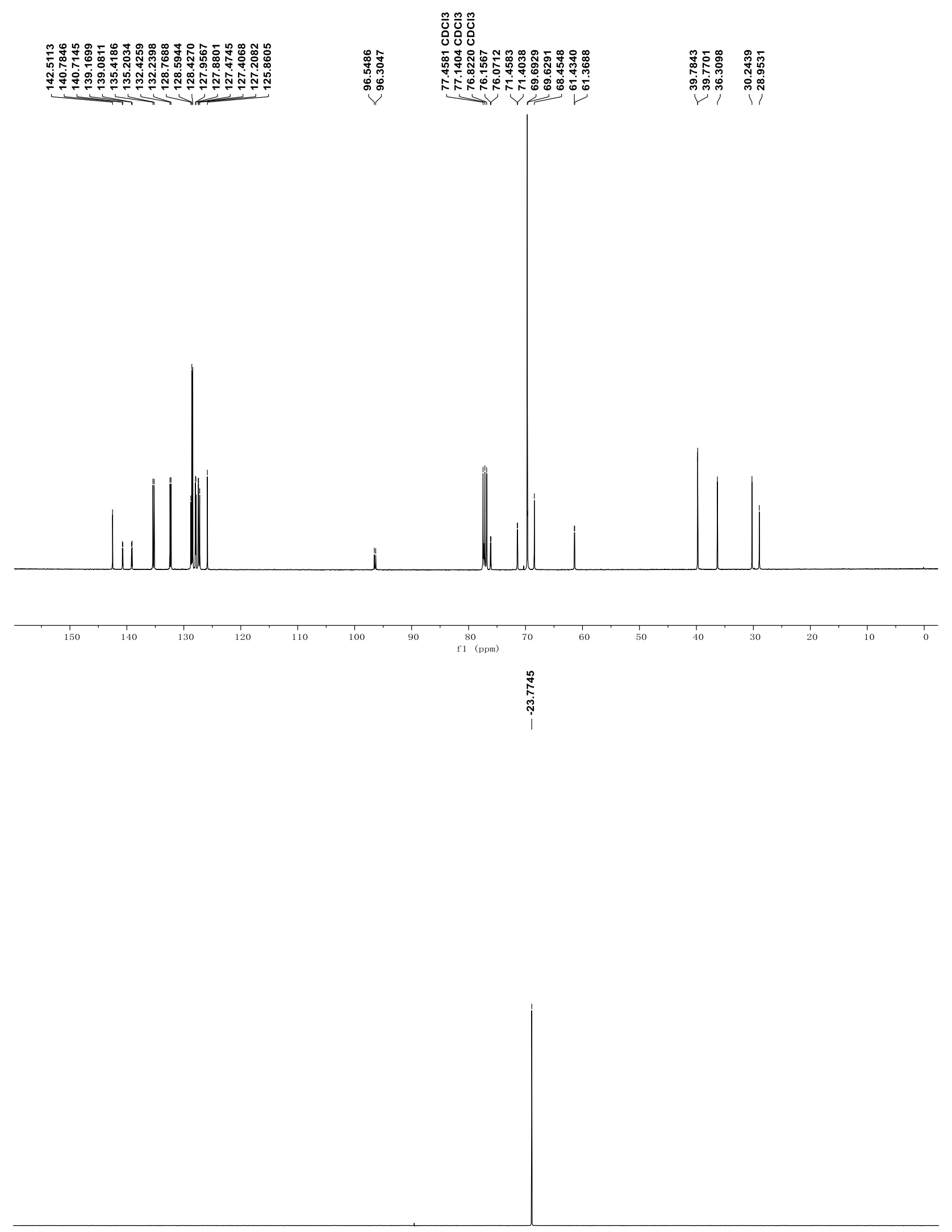

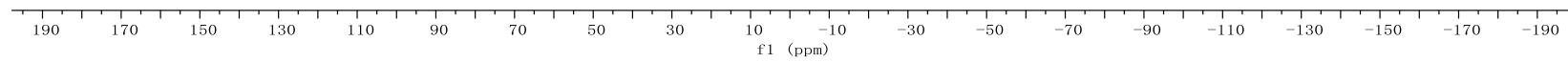


<smiles>N=C(C(=O)c1ccccc1)c1ccccc1</smiles>

$(S, R p-L 3)$

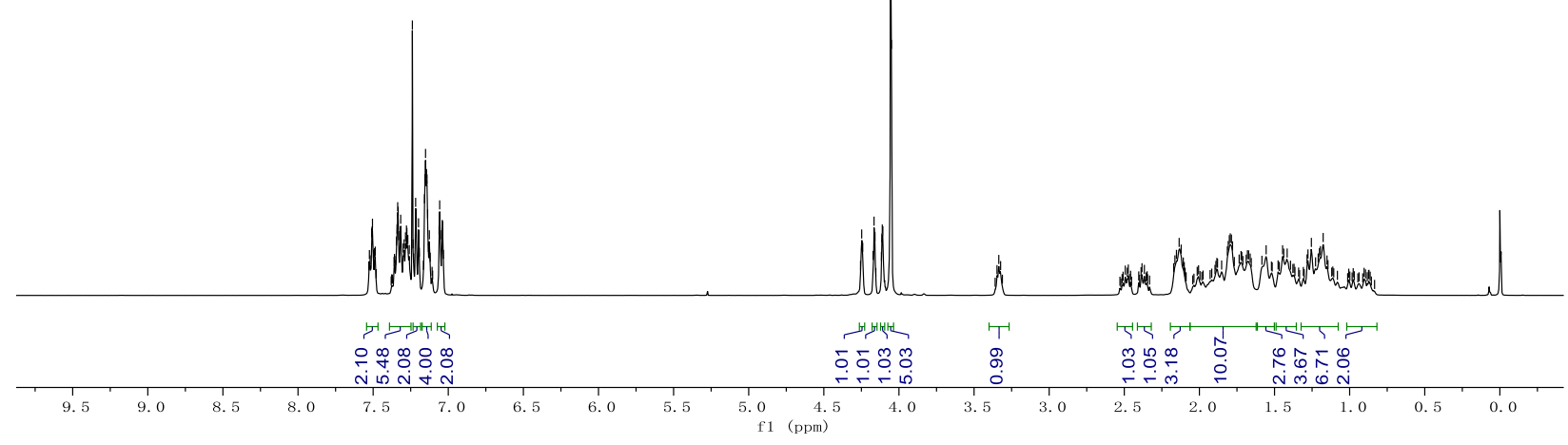

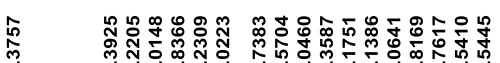

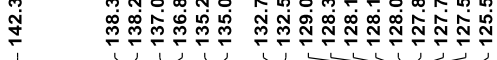

ए广
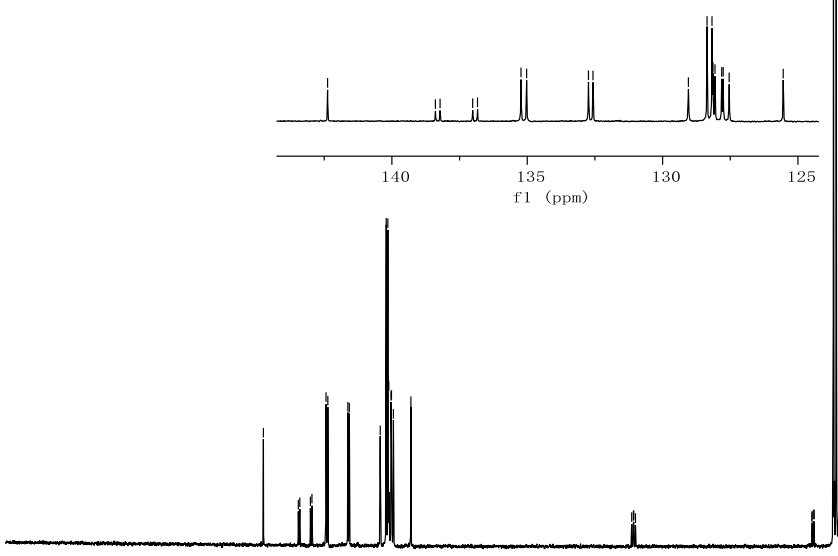

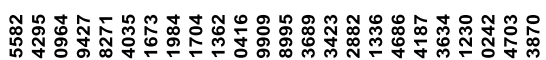

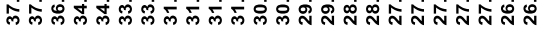

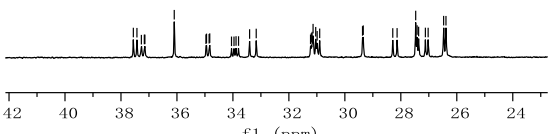

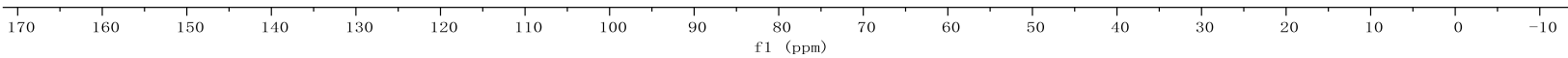




$$
\text { 紧 }
$$

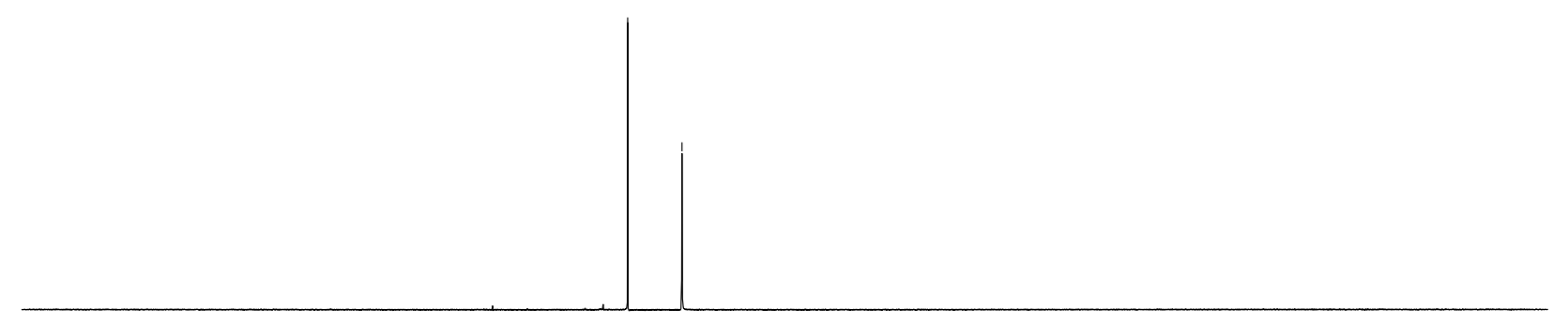

\begin{tabular}{|c|c|c|c|c|}
\hline 200 & 150 & 100 & 50 & 0 \\
\hline
\end{tabular}

\section{กำ}

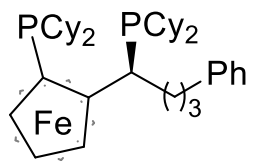

(S,Rp-L4)

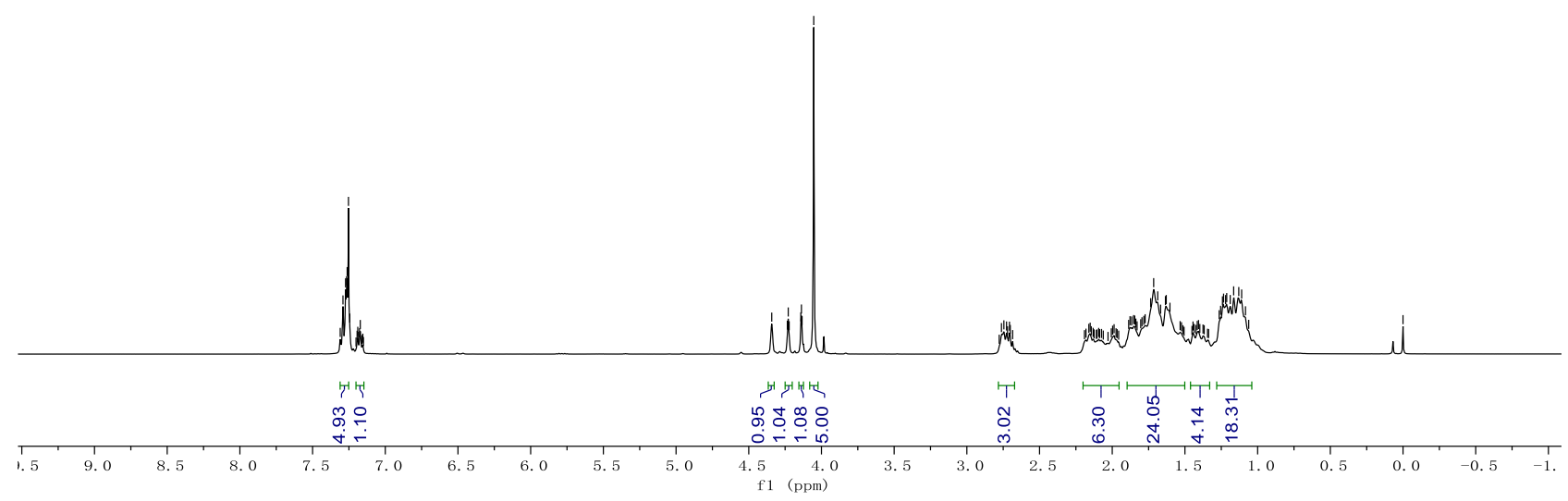



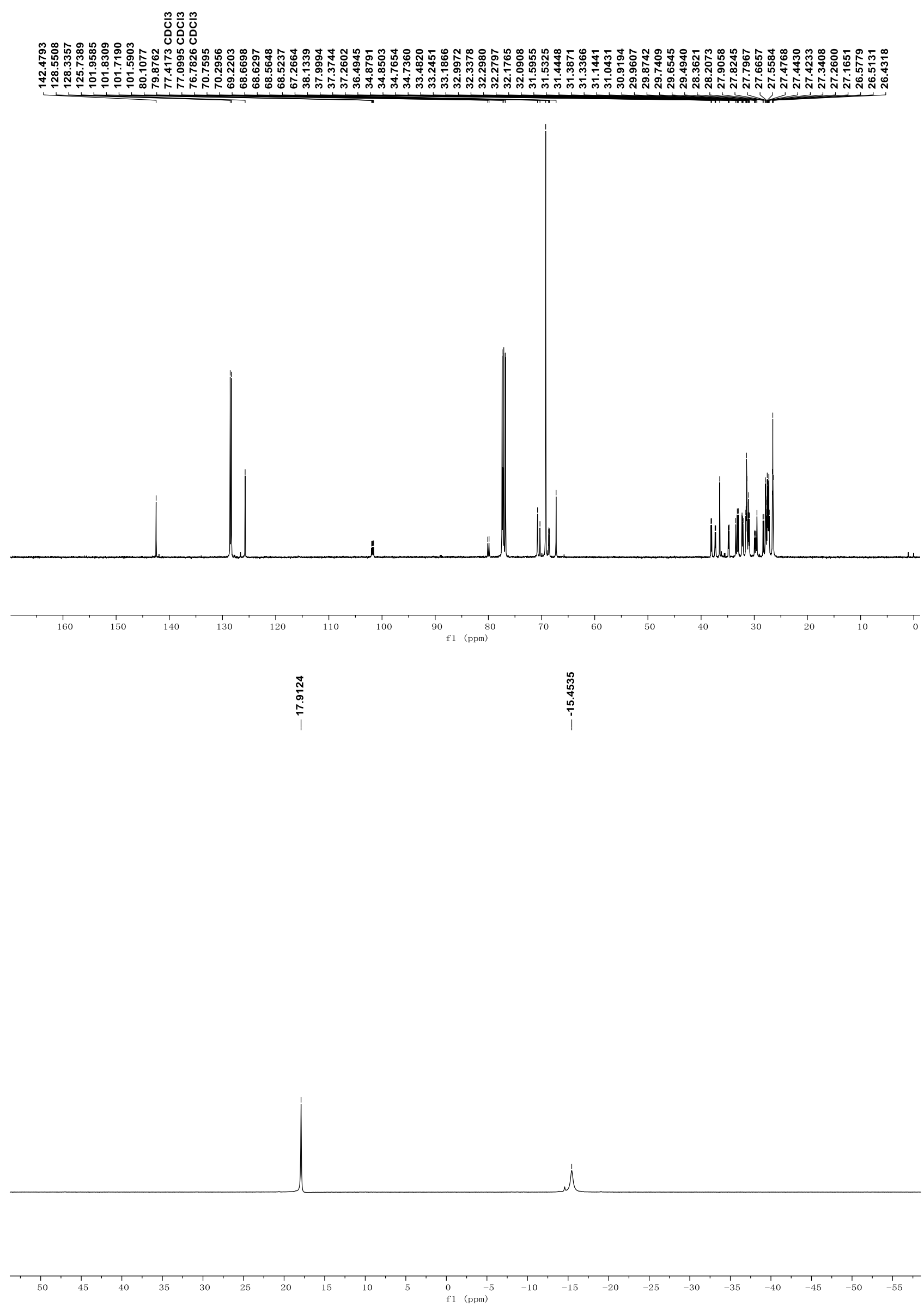
品

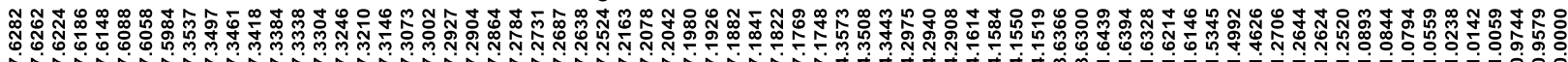<smiles>Pc1ccccc1</smiles>

$(S, R p-L 5)$
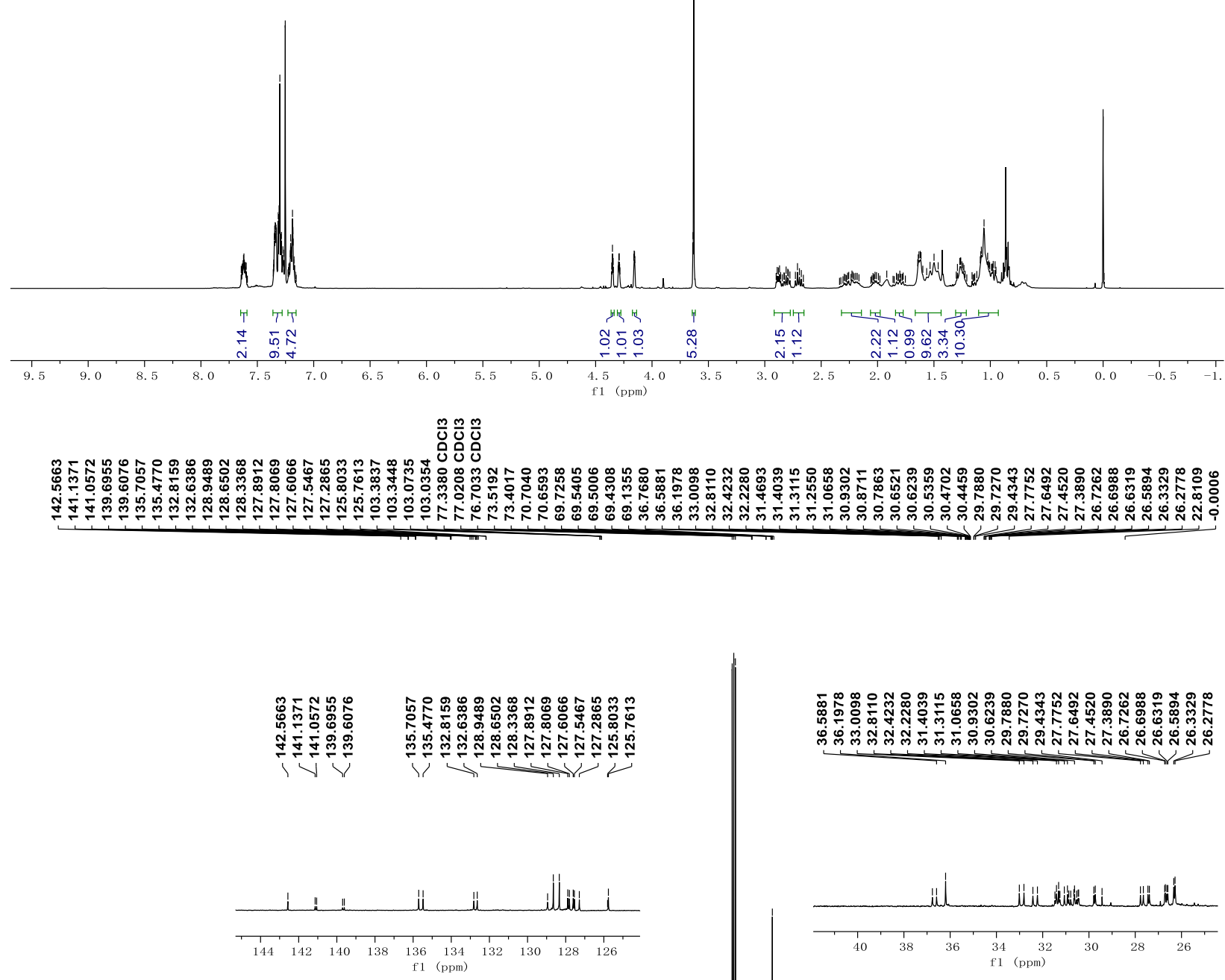


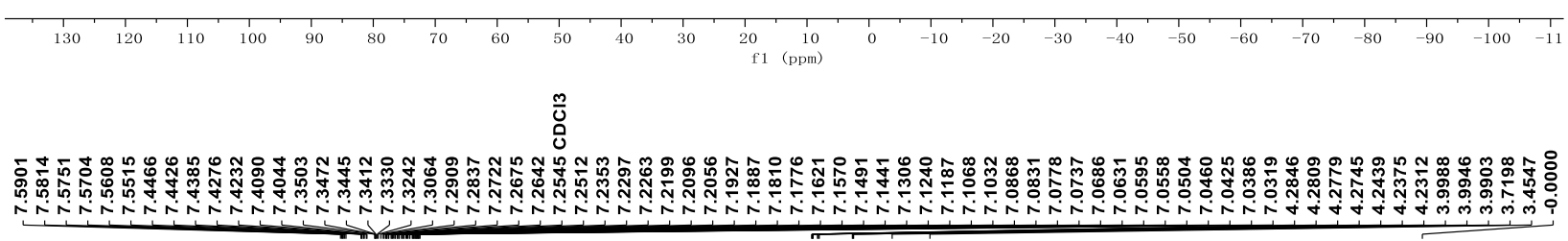

$$
\text { Fe] }
$$

(S,Rp-L6)

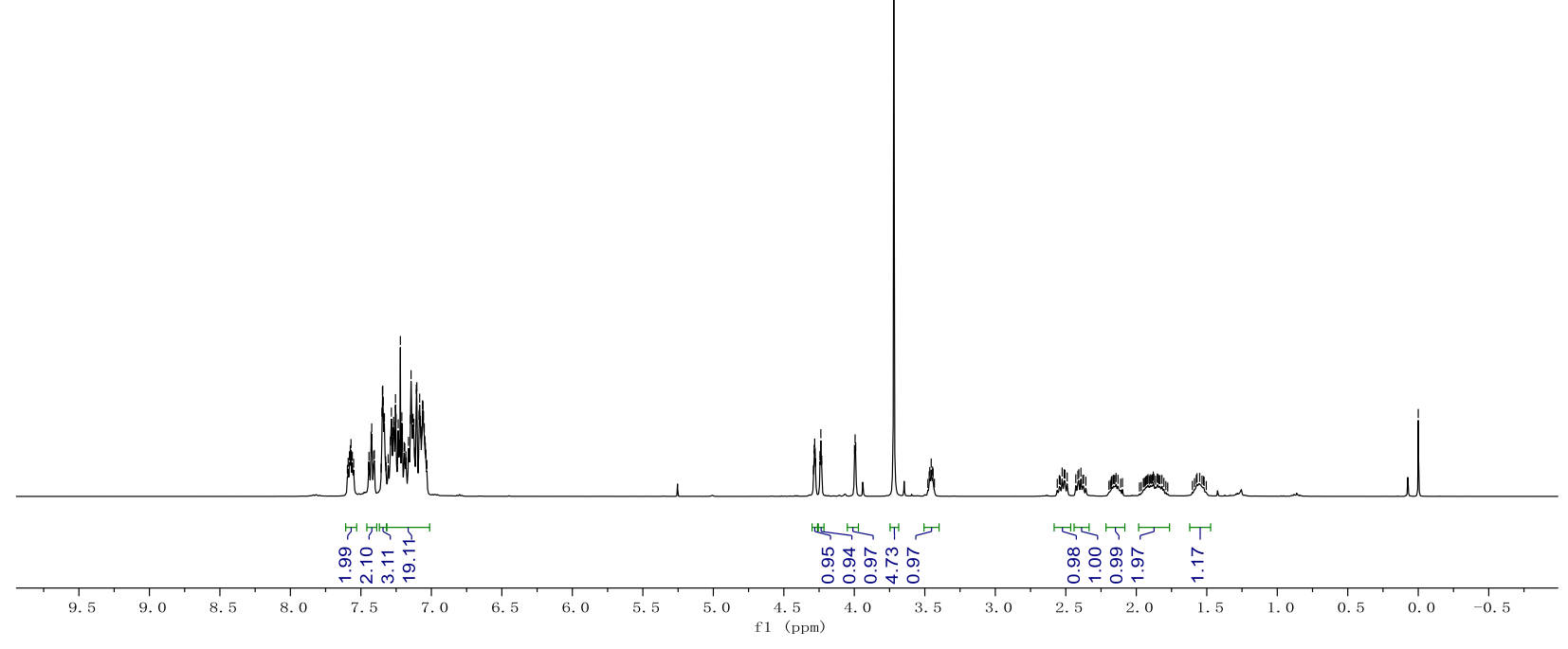




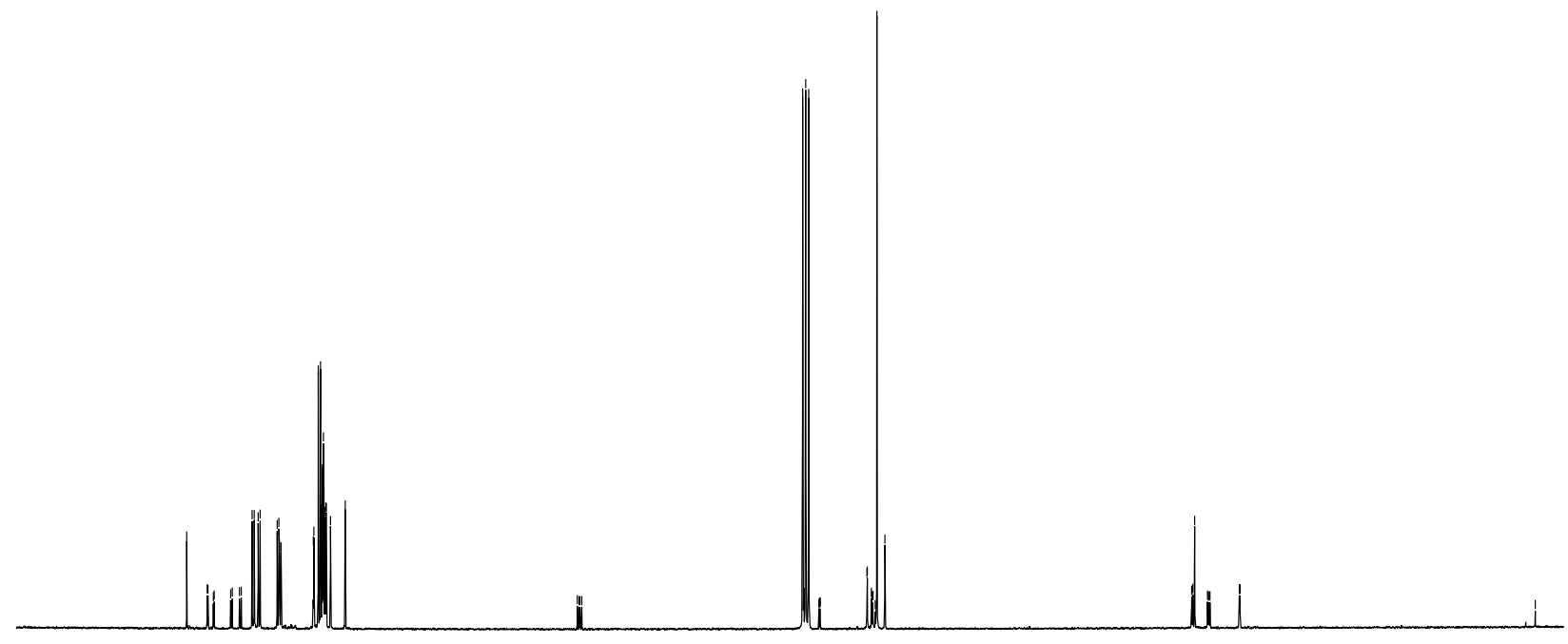

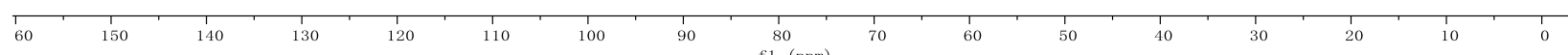

ن

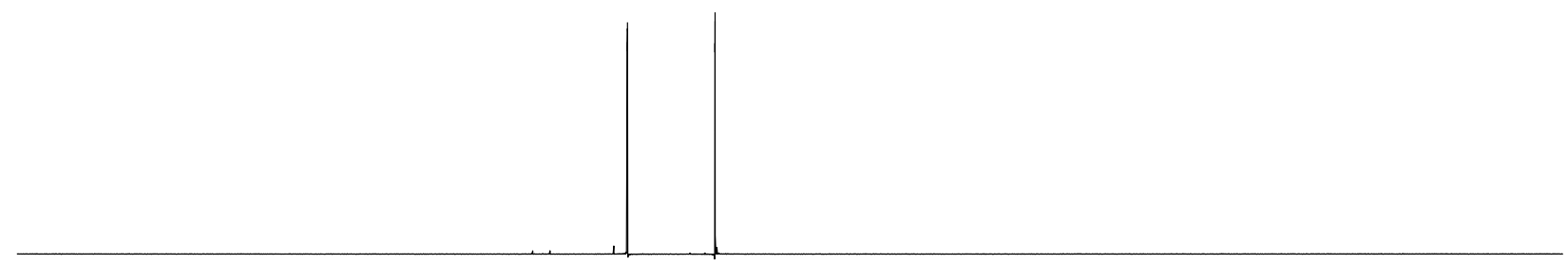

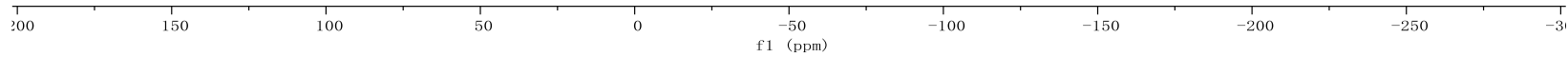



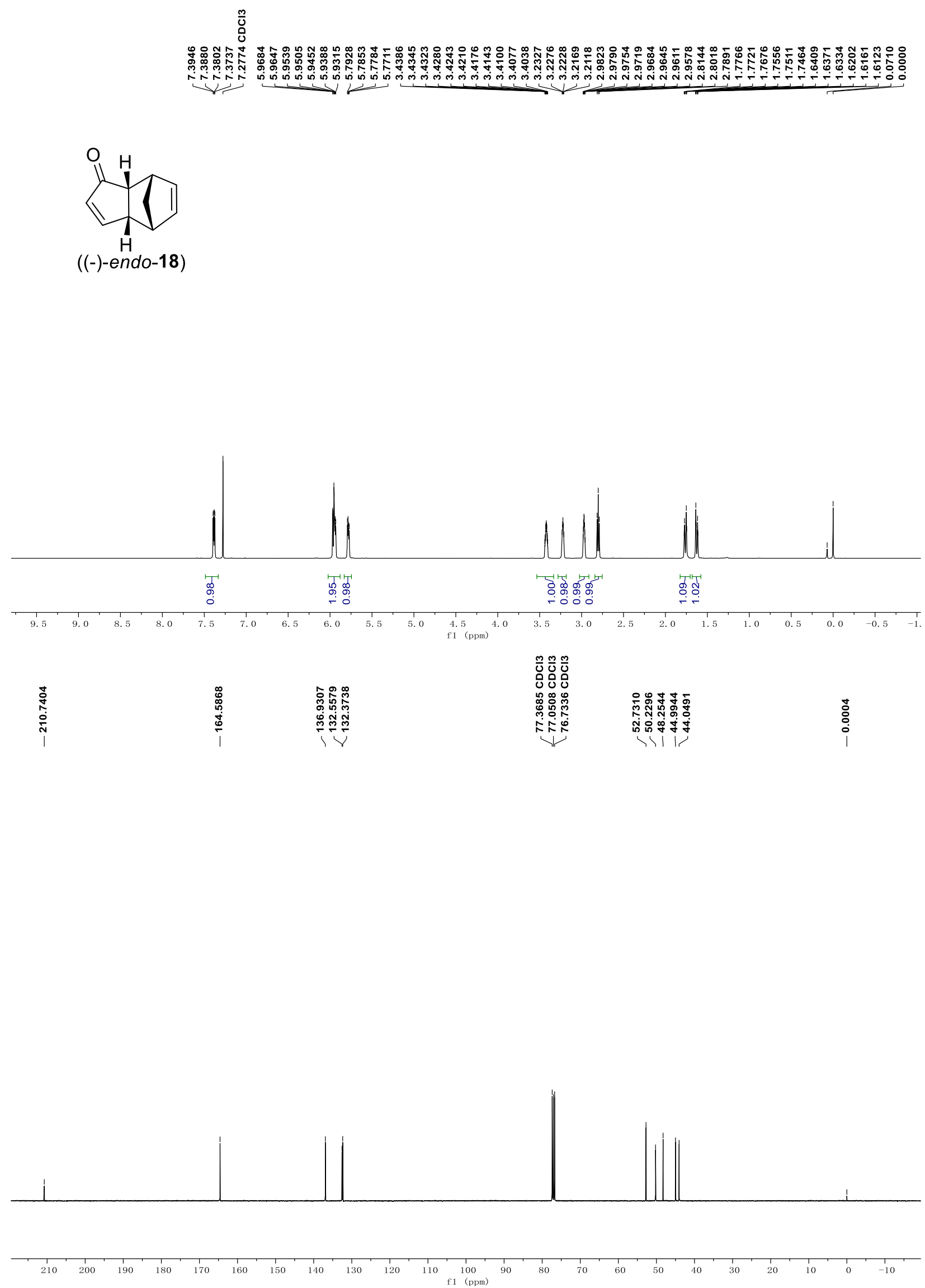


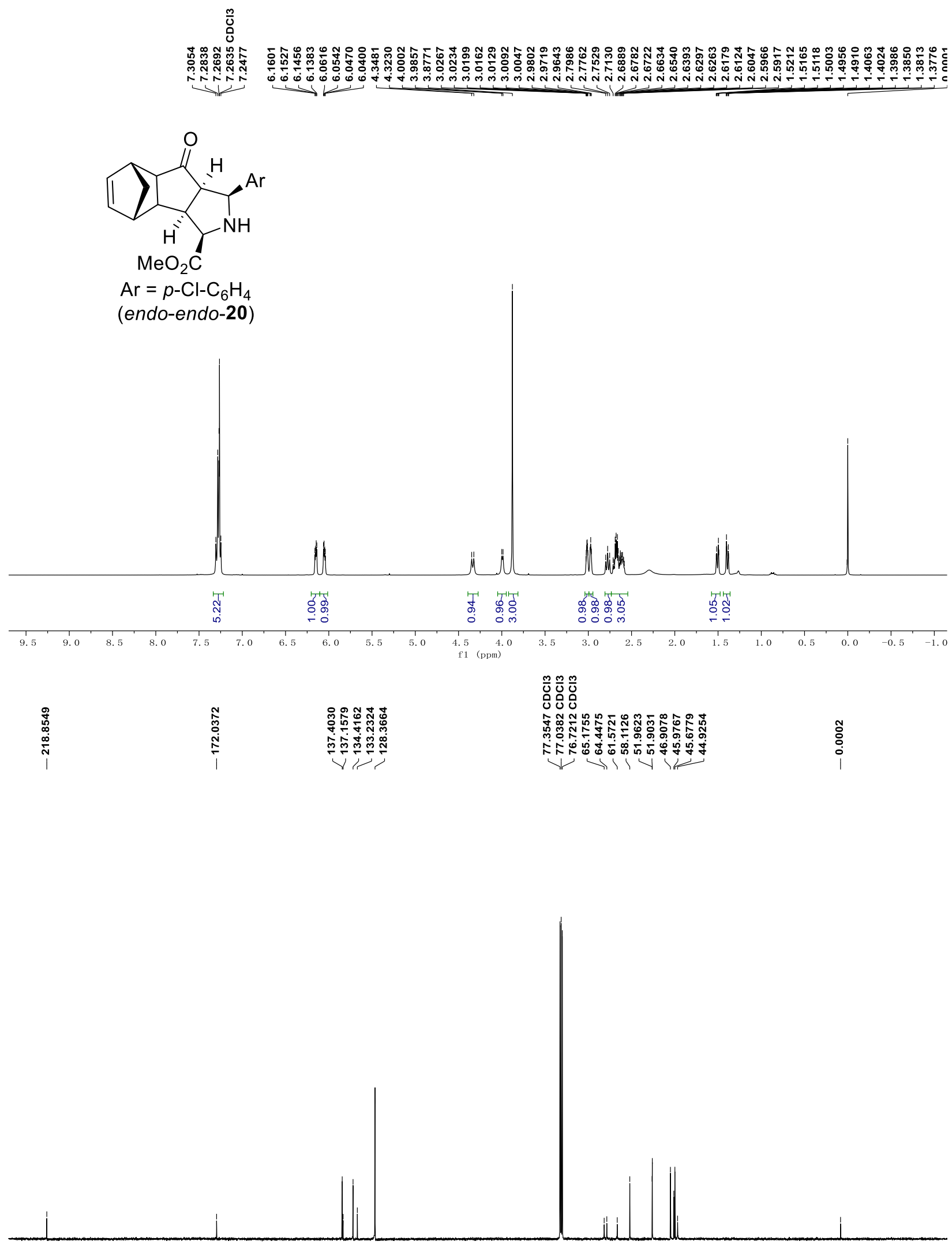

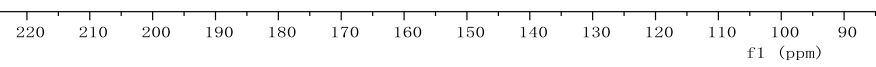




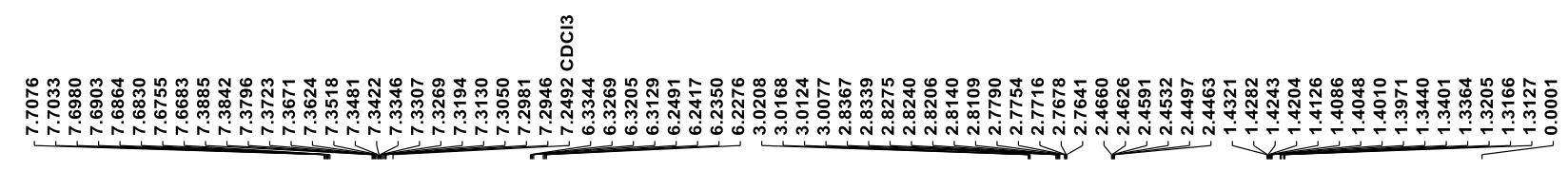<smiles>O=C1C(c2ccccc2)=CC2C3C=CC(C3)C12</smiles>

((-)-exo-21)

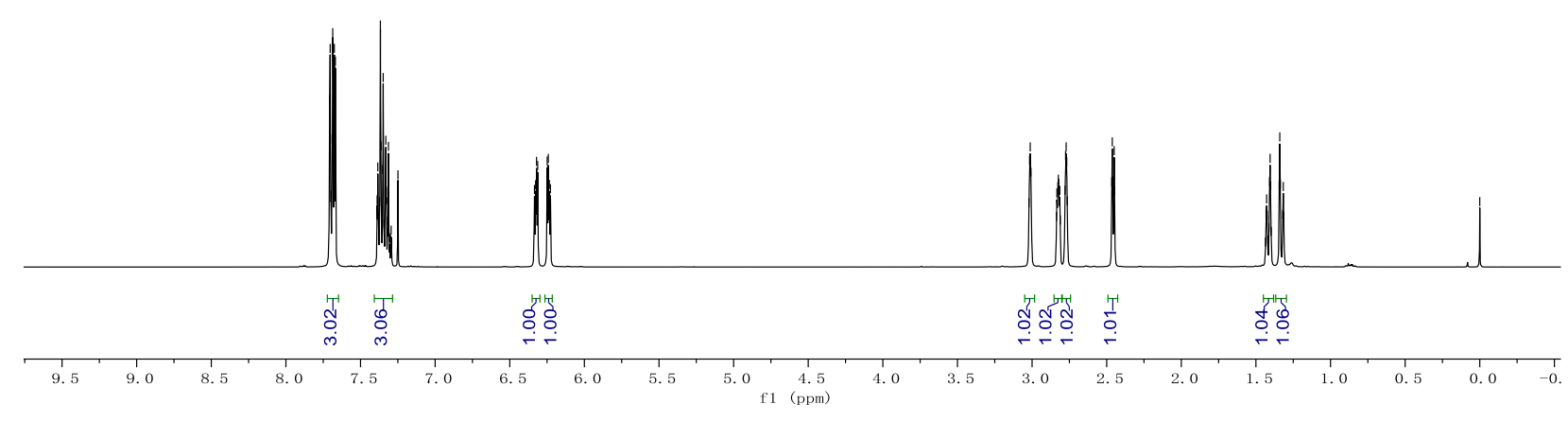

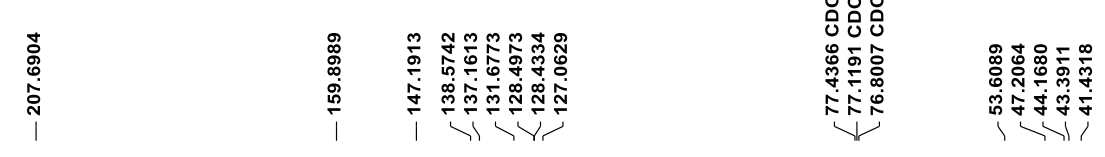

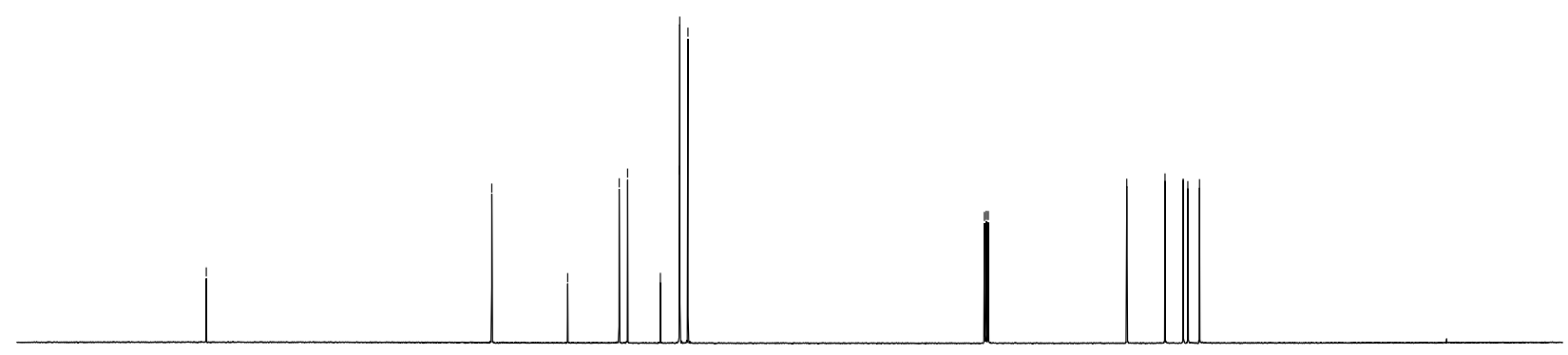

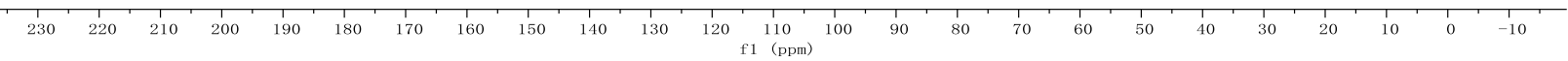


$\frac{m}{0.0}$

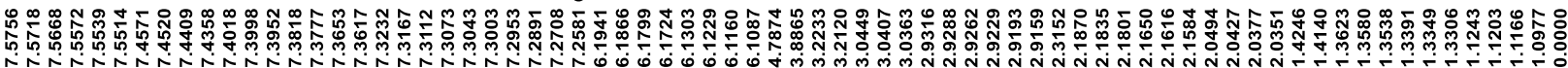

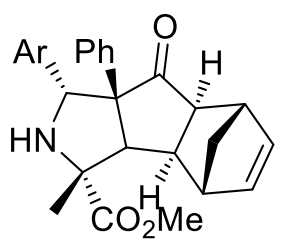

$\mathrm{Ar}=p-\mathrm{Cl}-\mathrm{C}_{6} \mathrm{H}_{4}$

(endo-exo-22)

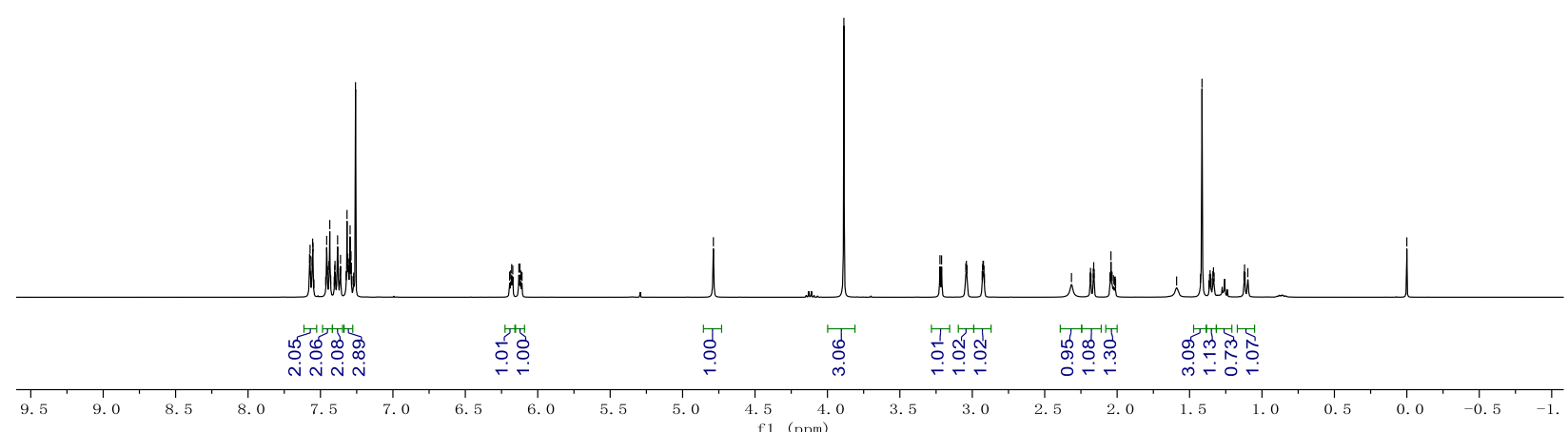

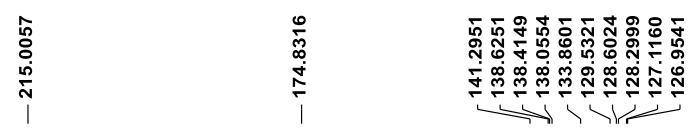

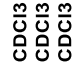

$\begin{array}{ll} & \\ 0 & \end{array}$

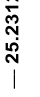

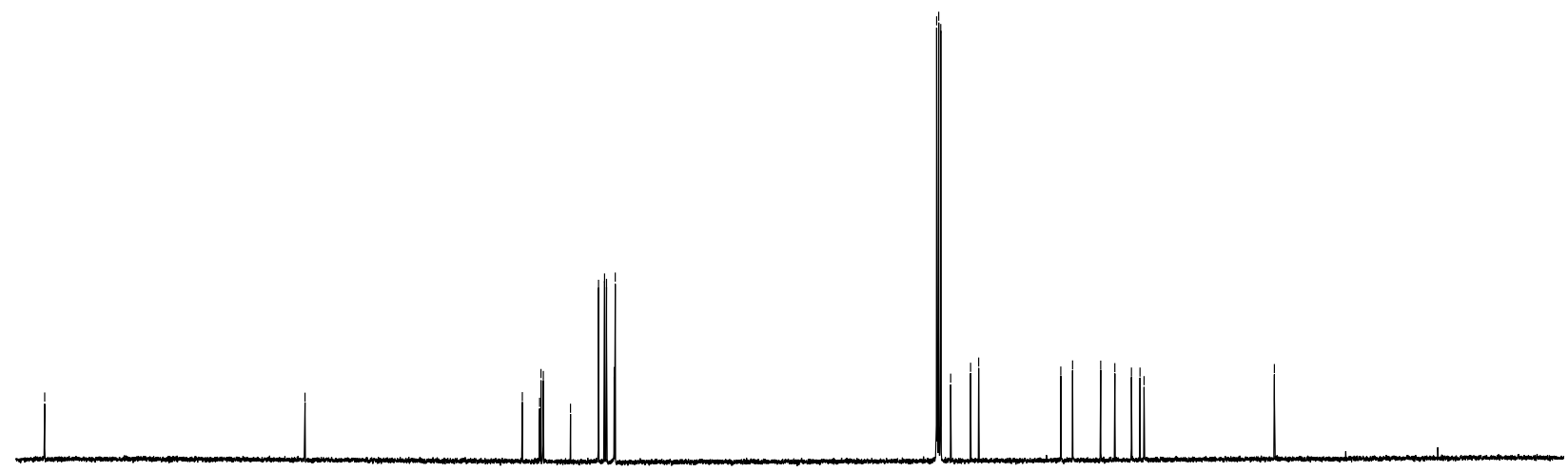

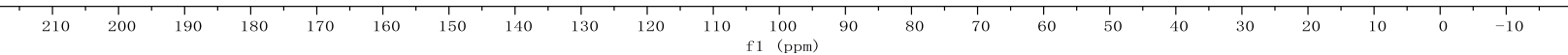




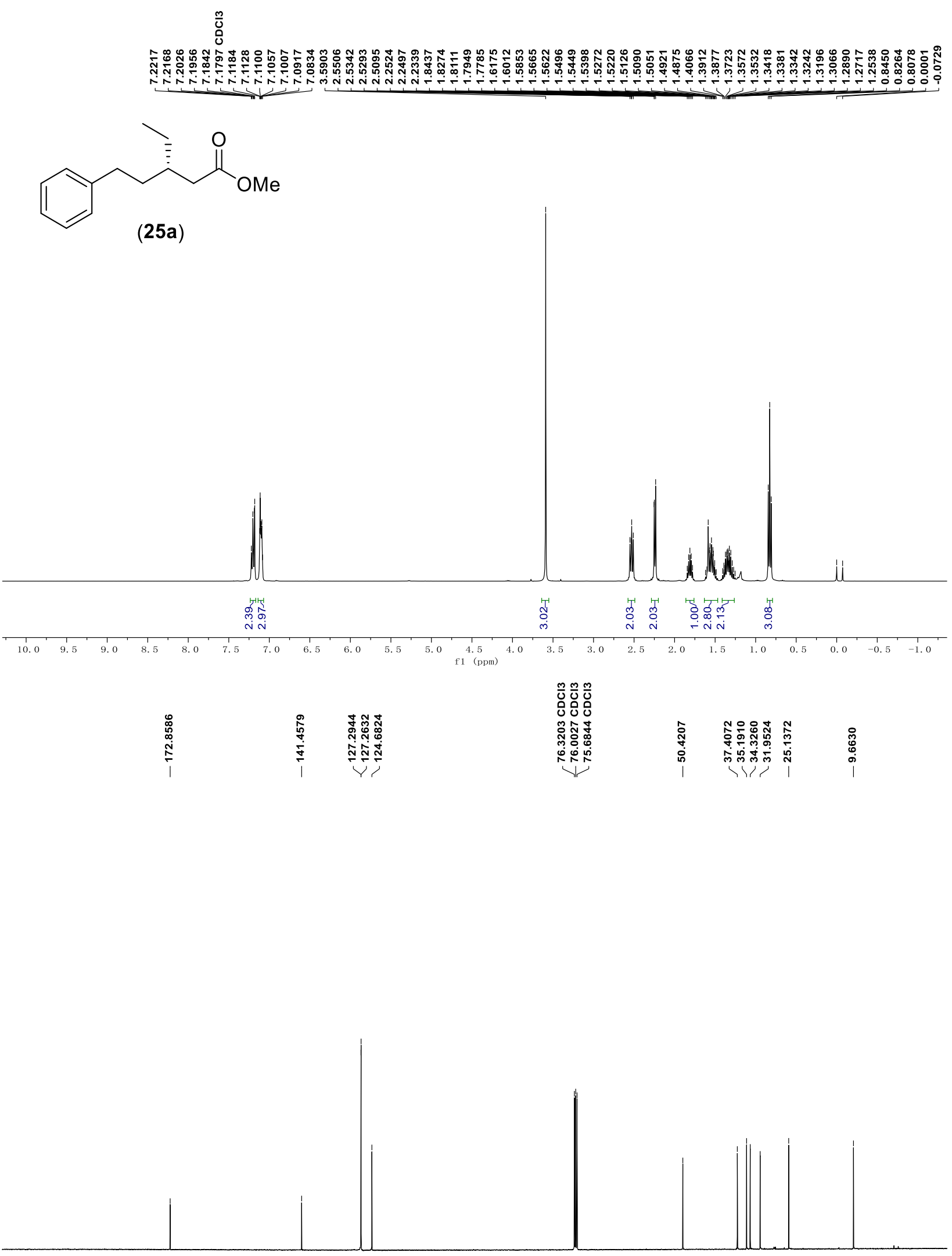

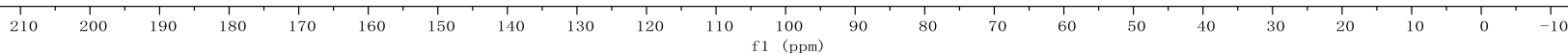



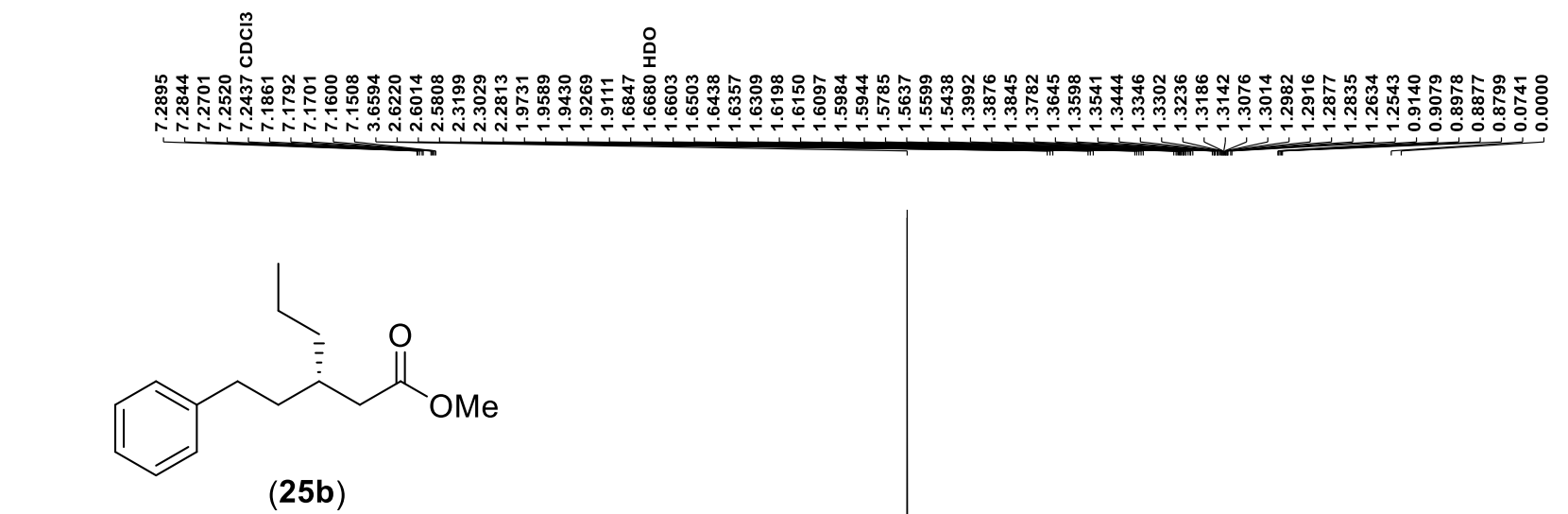

(25b)
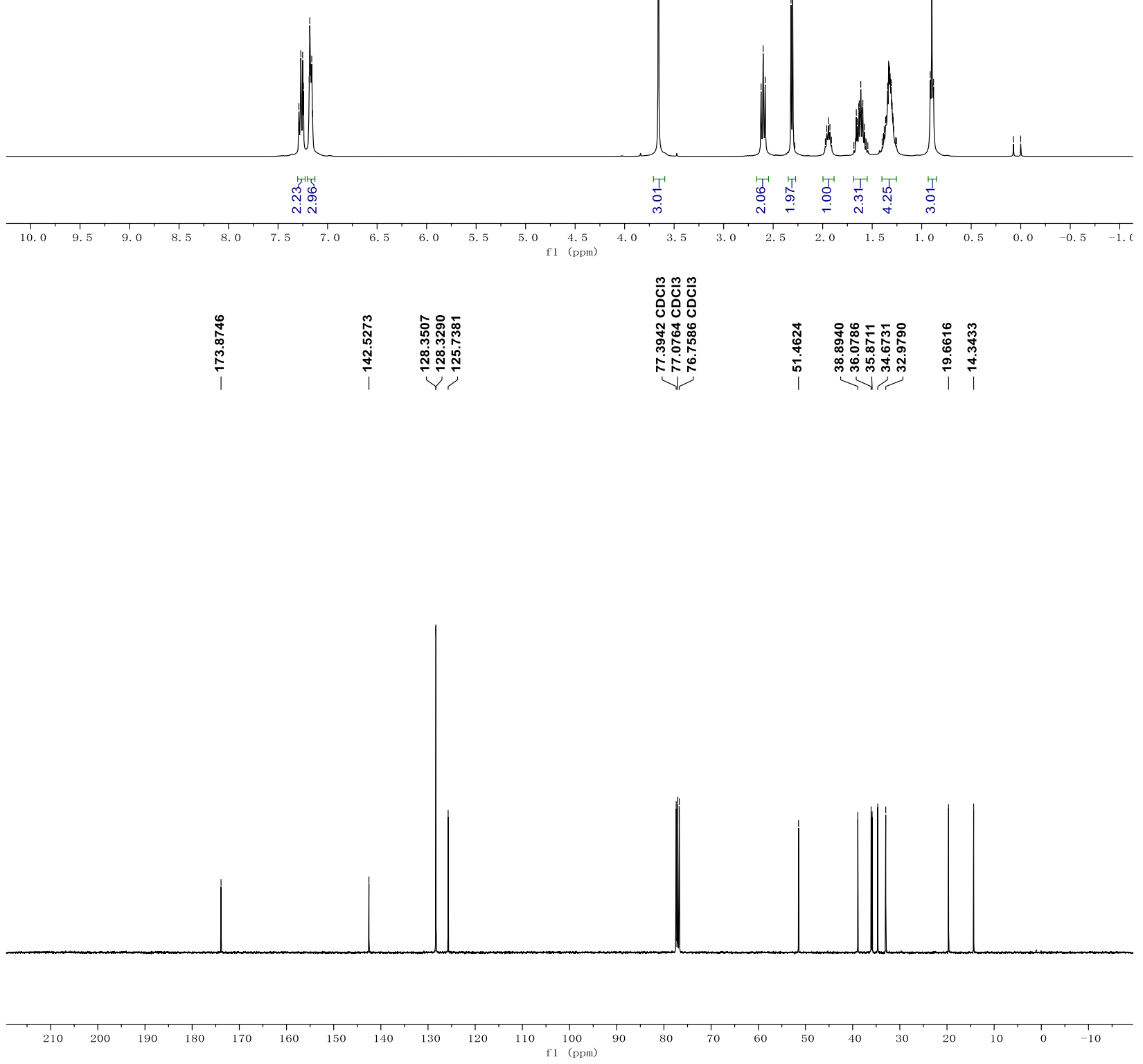

S56 


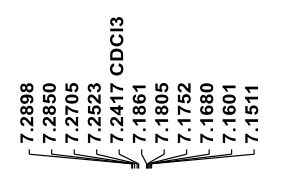

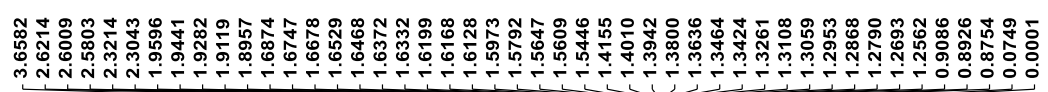<smiles>CCCC[C@H](CCc1ccccc1)CC(=O)OC</smiles>

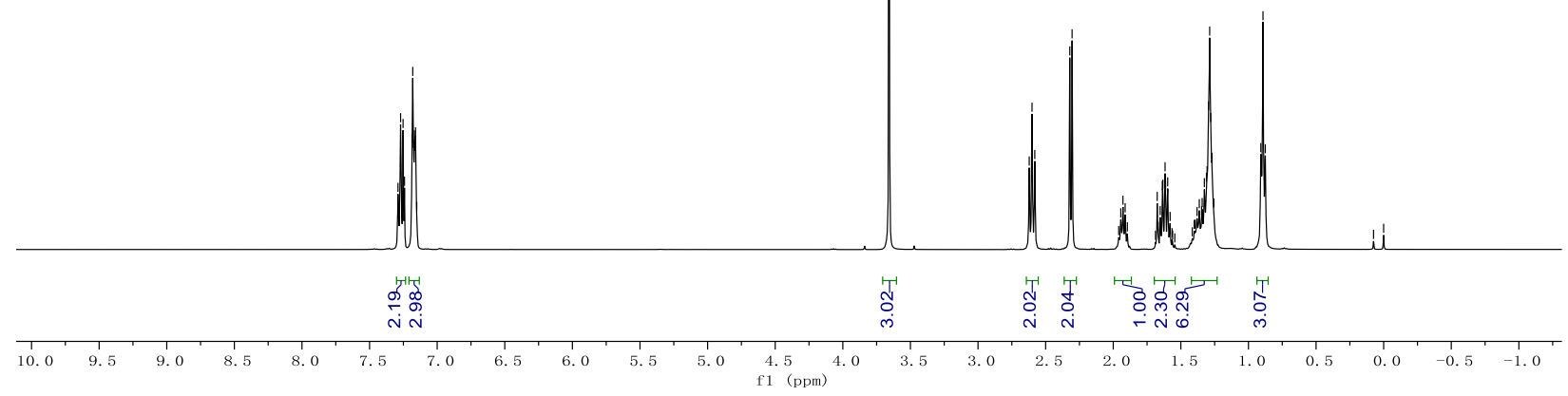

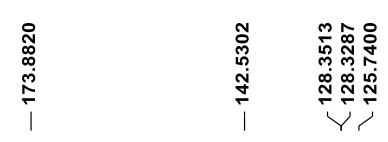
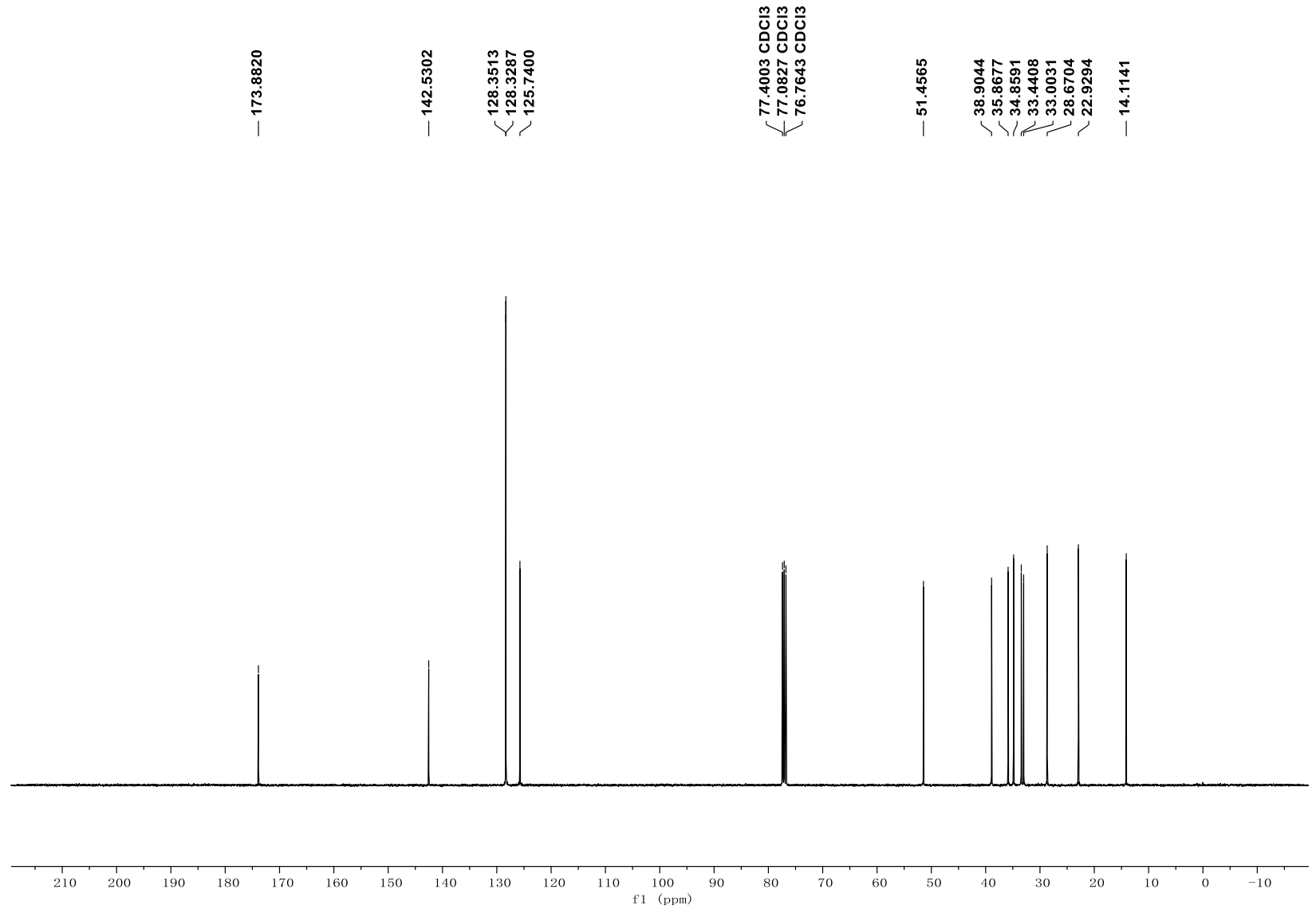
$\stackrel{\frac{m}{0}}{0}$

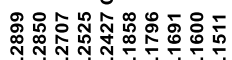

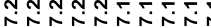
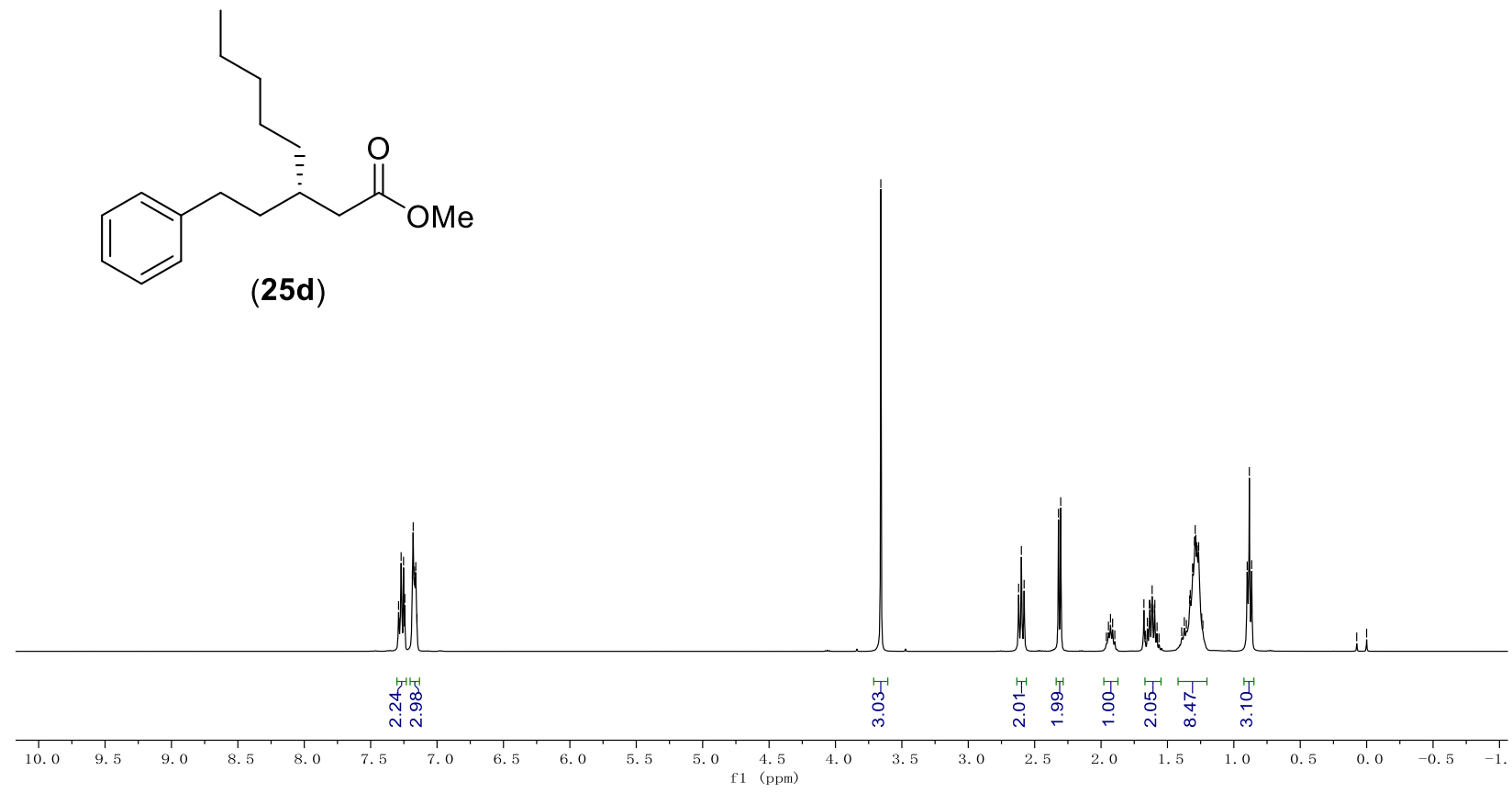

$\stackrel{\circ}{\stackrel{1}{x}}$

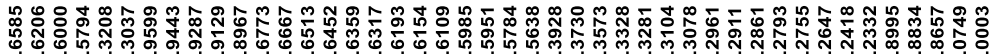

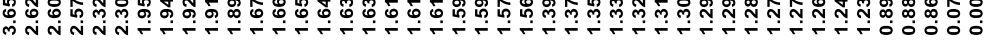

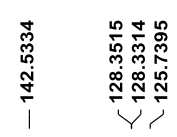

它芦
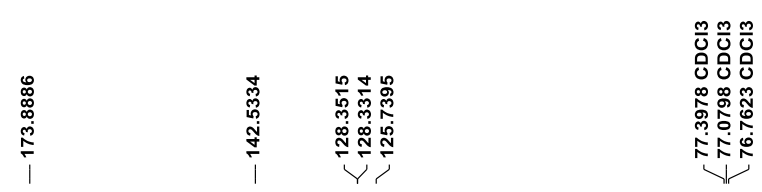

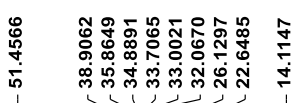

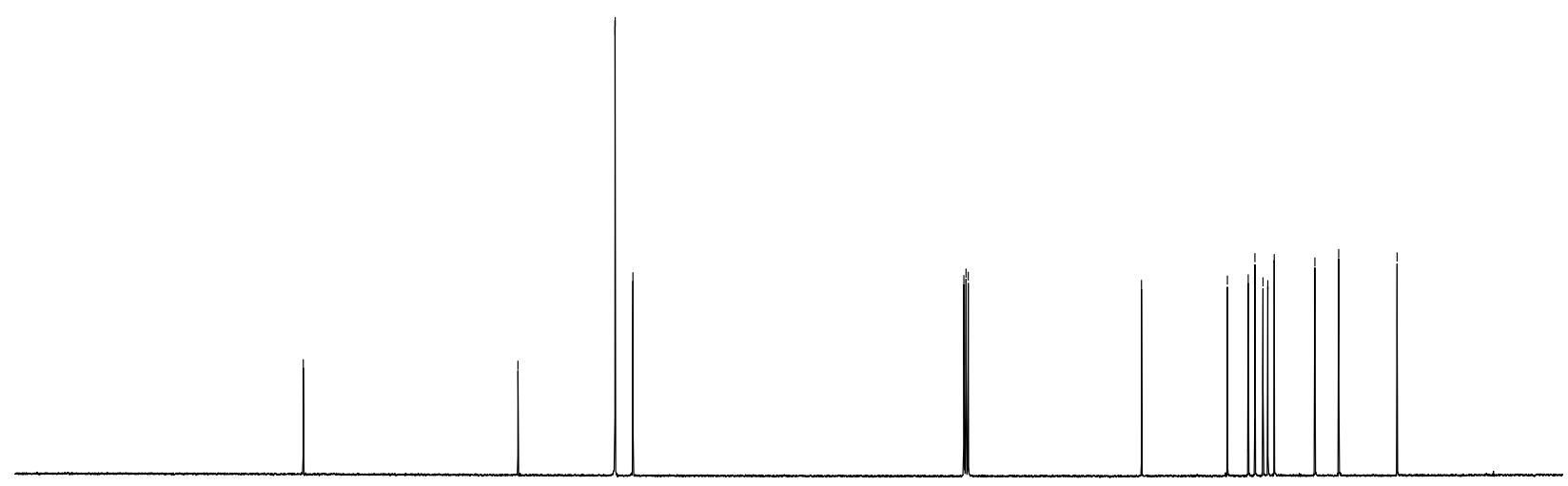

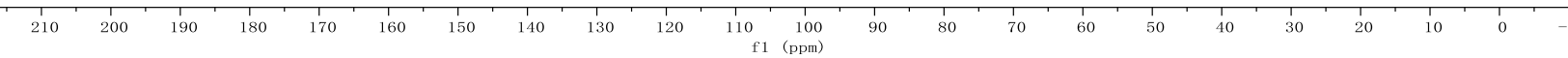




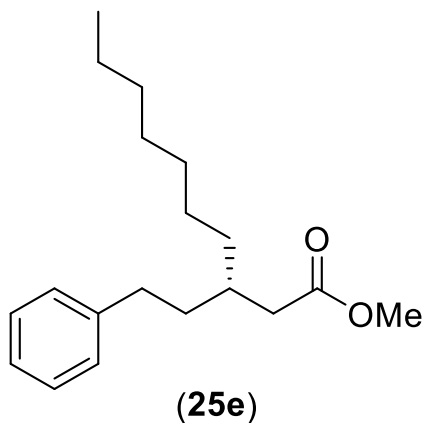

(25e)
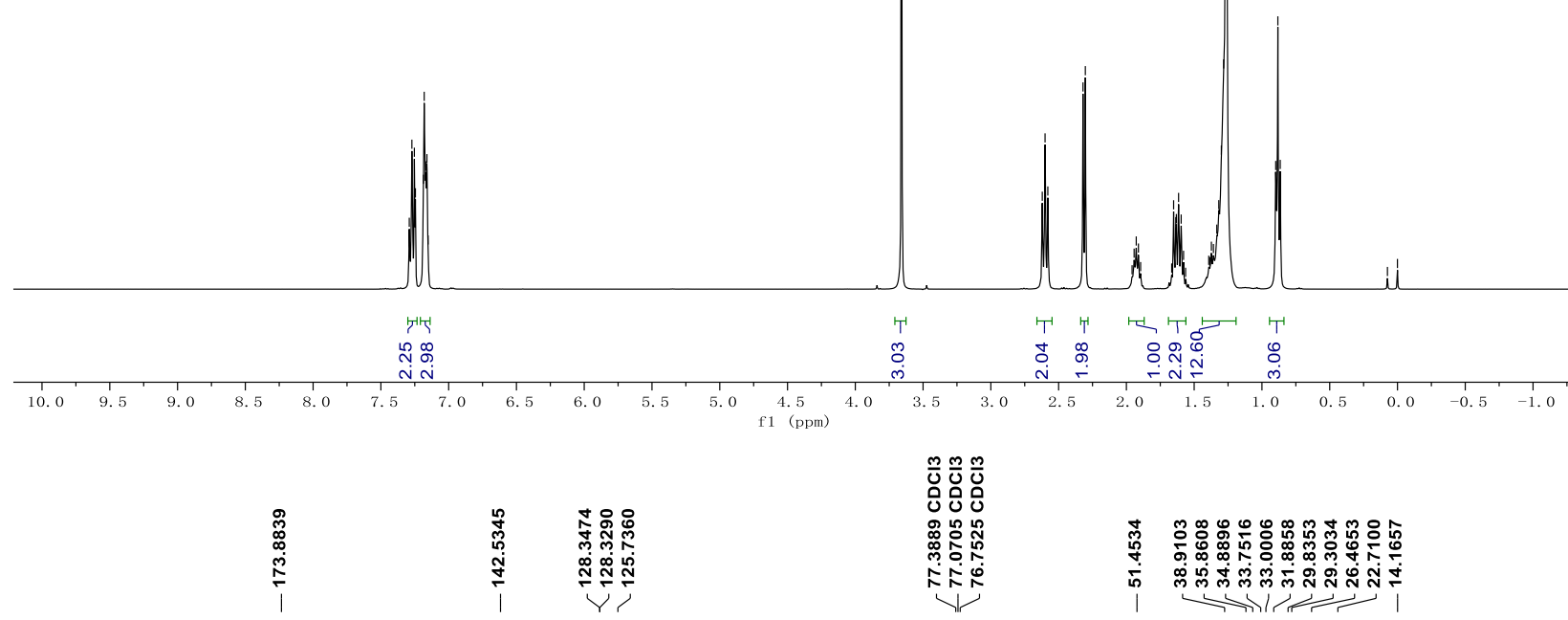

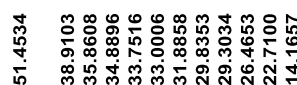

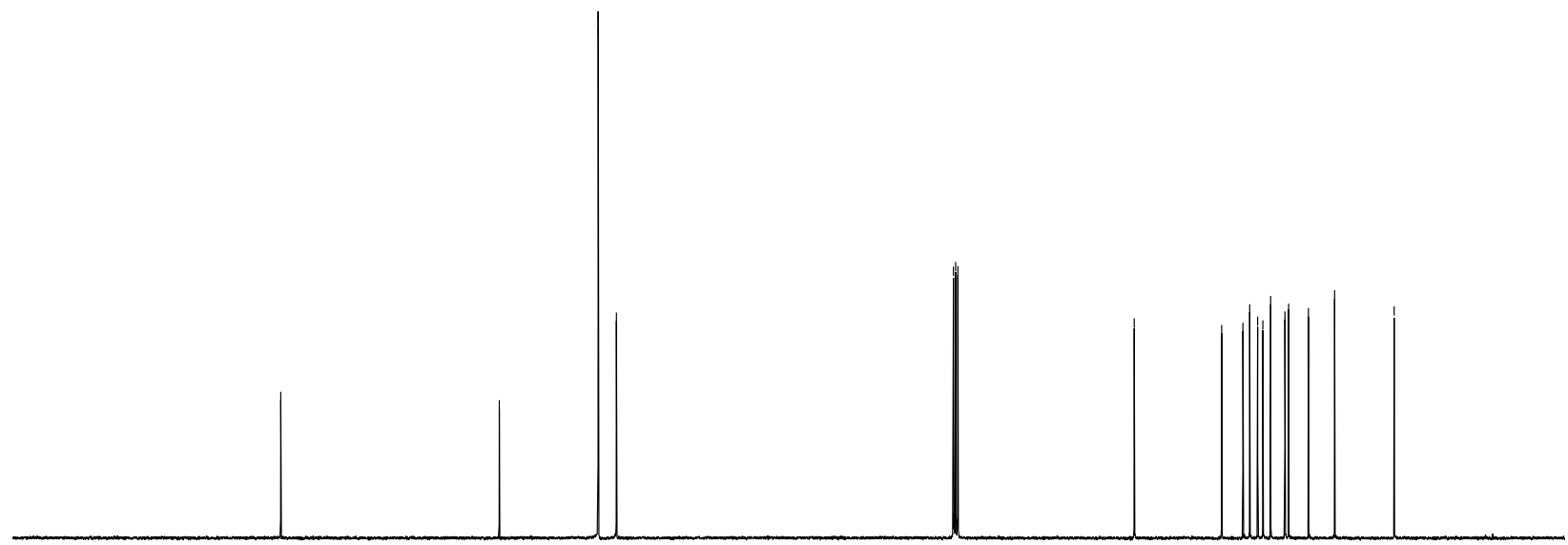

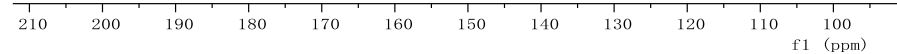


<smiles>COC(=O)C[C@H](C)CCc1ccccc1</smiles>

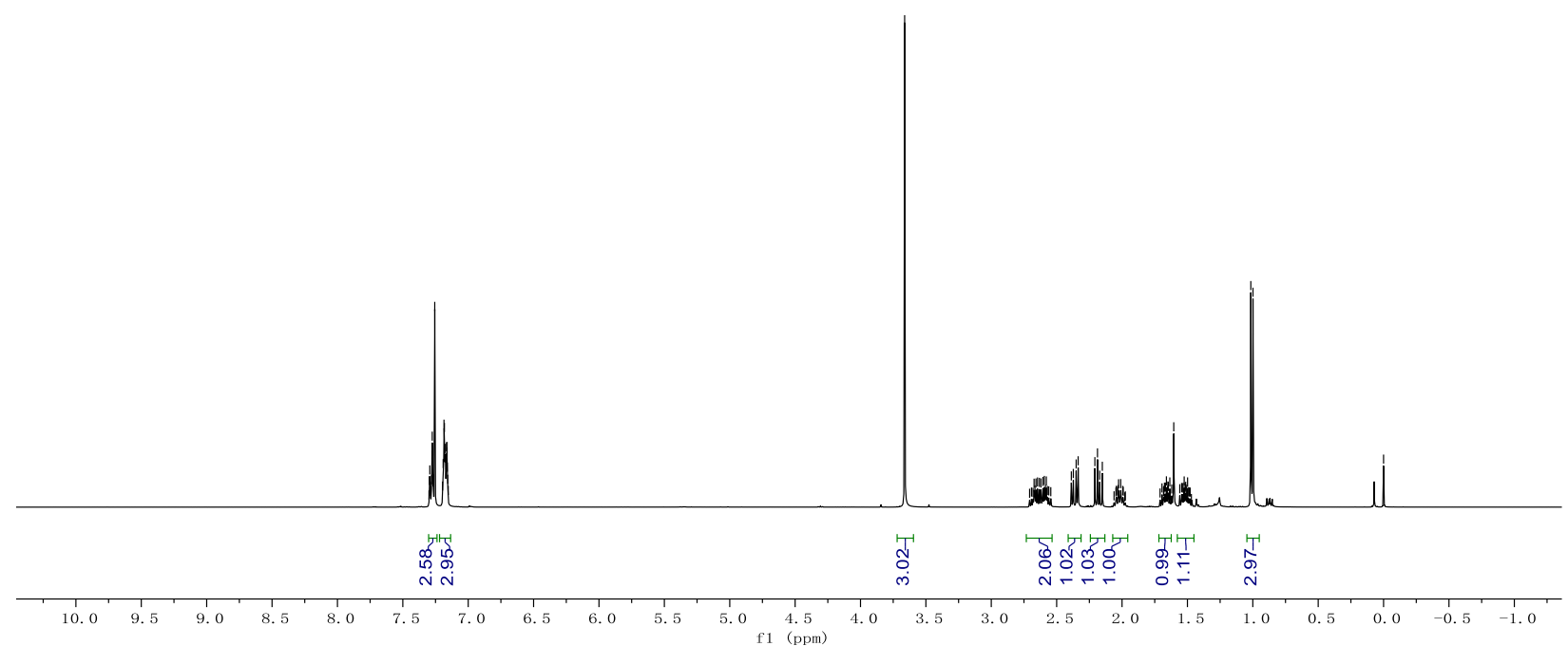

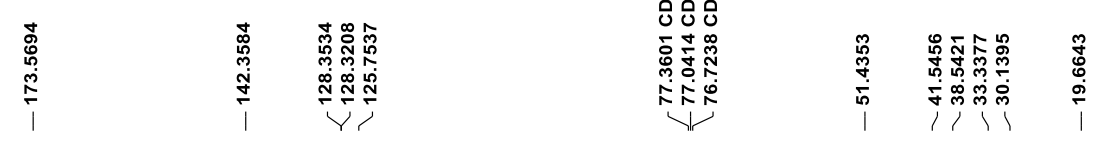
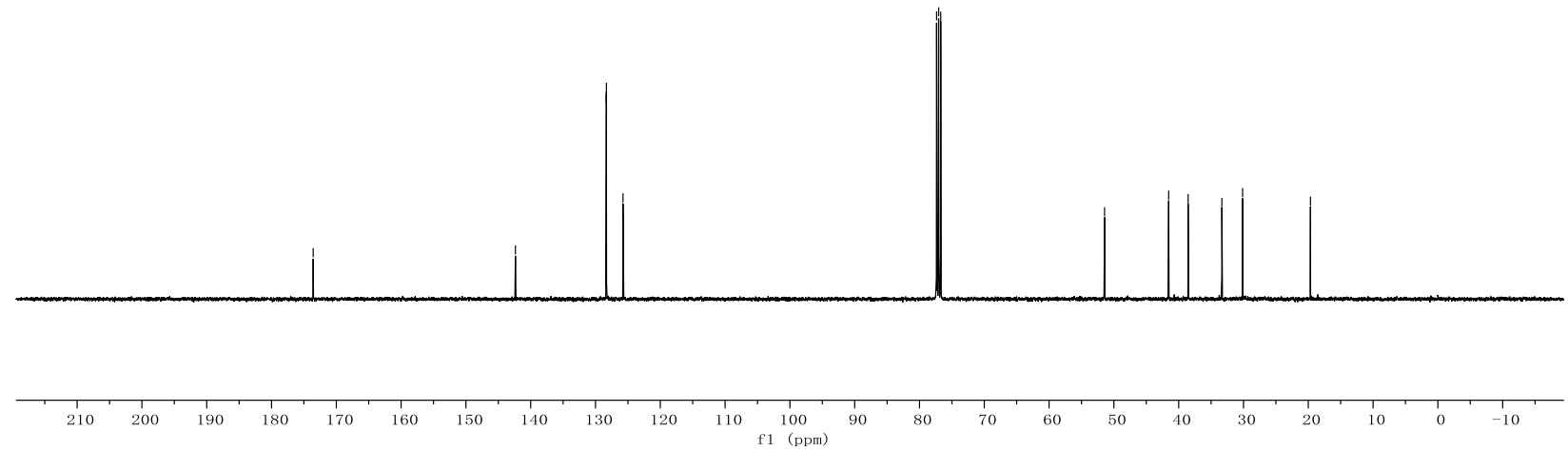
$\stackrel{m}{\mathrm{O}}$

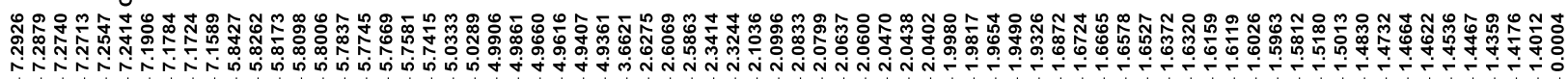

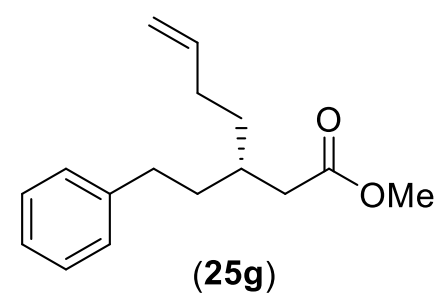

(25g)
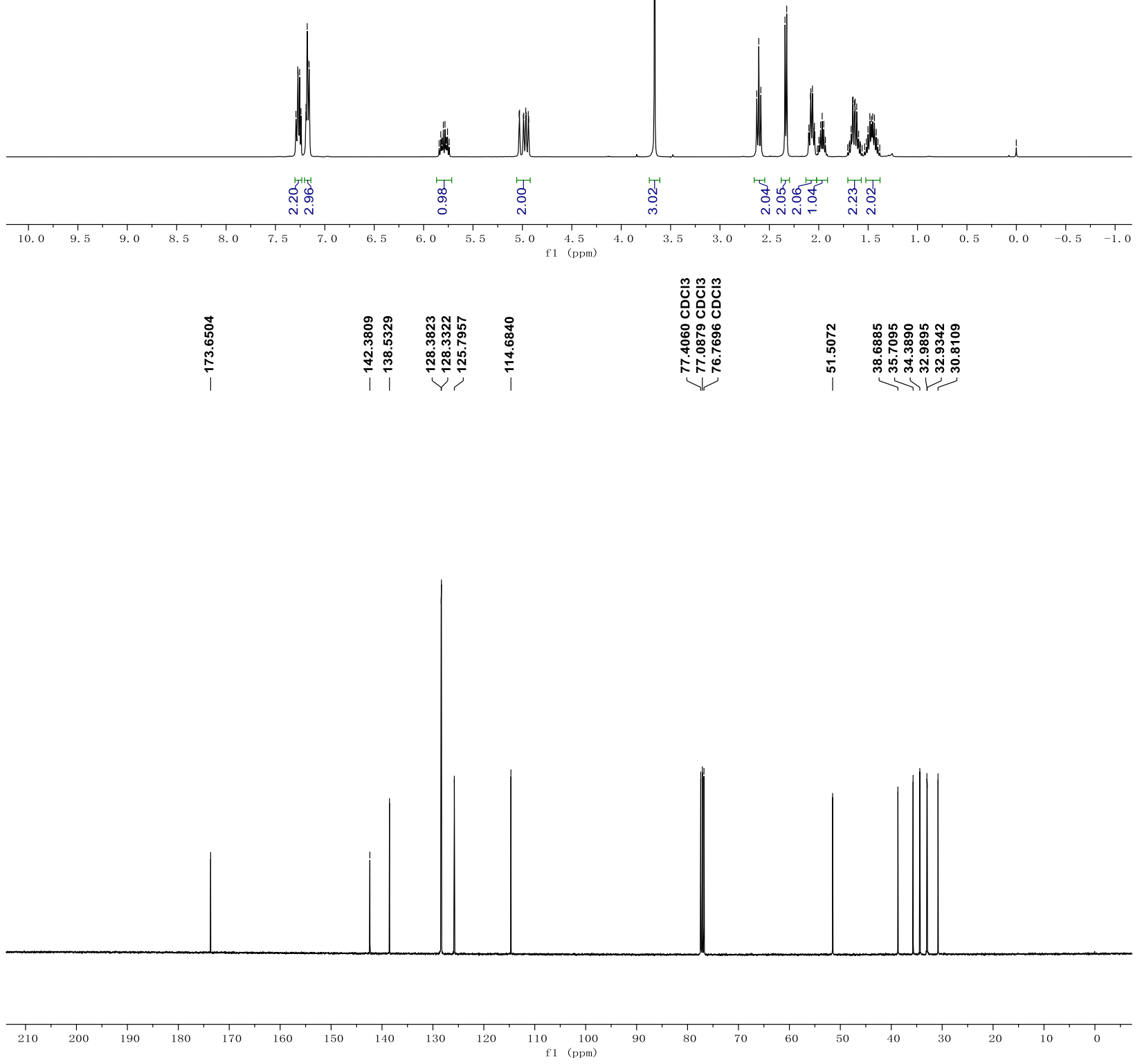

S61 


\section{$\frac{m}{0}$
0
0
$\stackrel{0}{N}$
$i$
$i$}

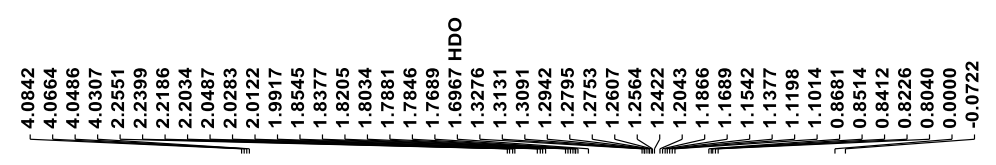

$\overbrace{(25 h)}^{\equiv}$
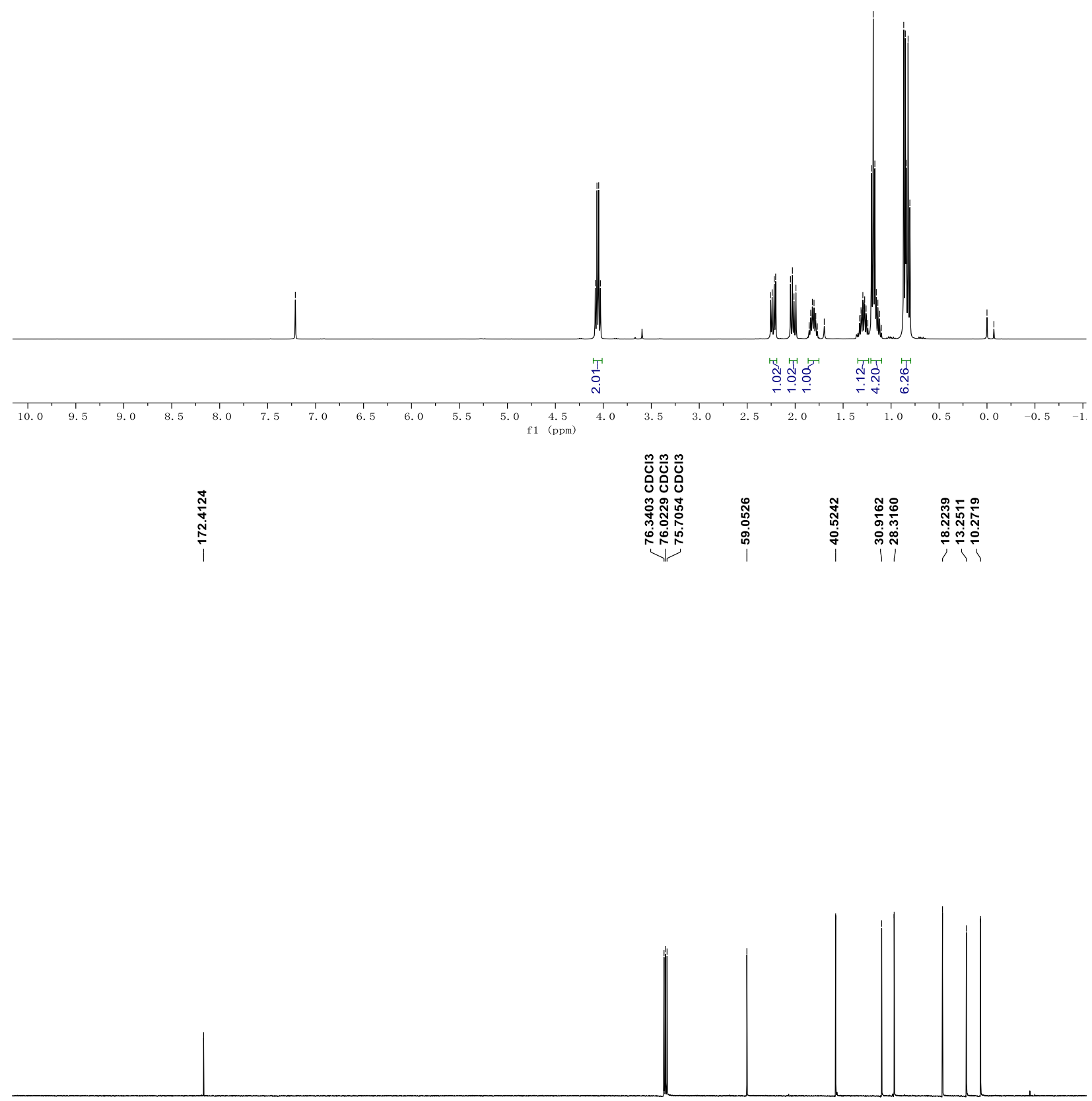

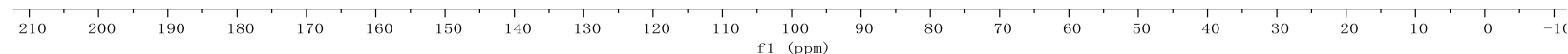



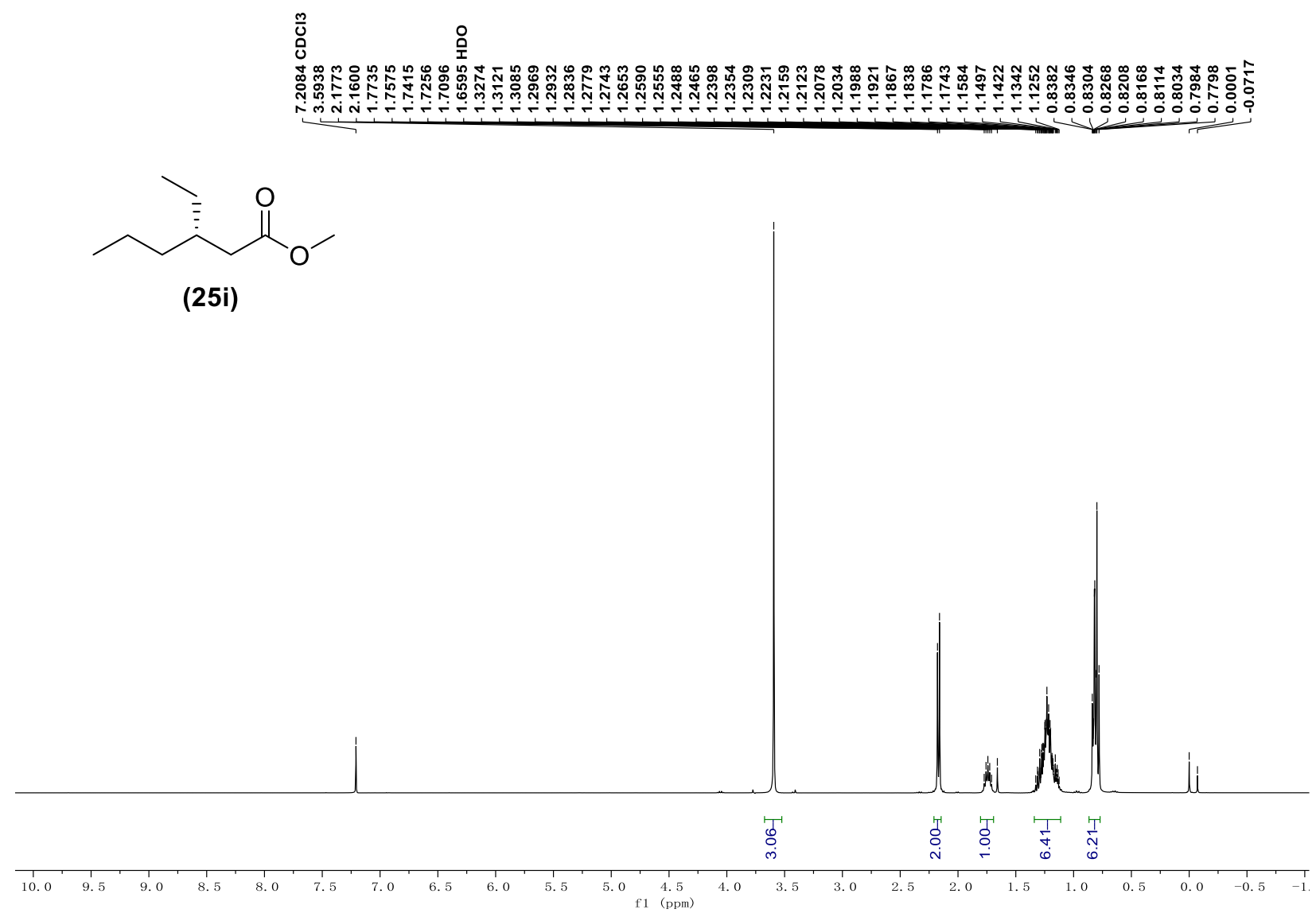

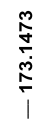

$\frac{m}{0} \frac{m}{0}$
0
0

1
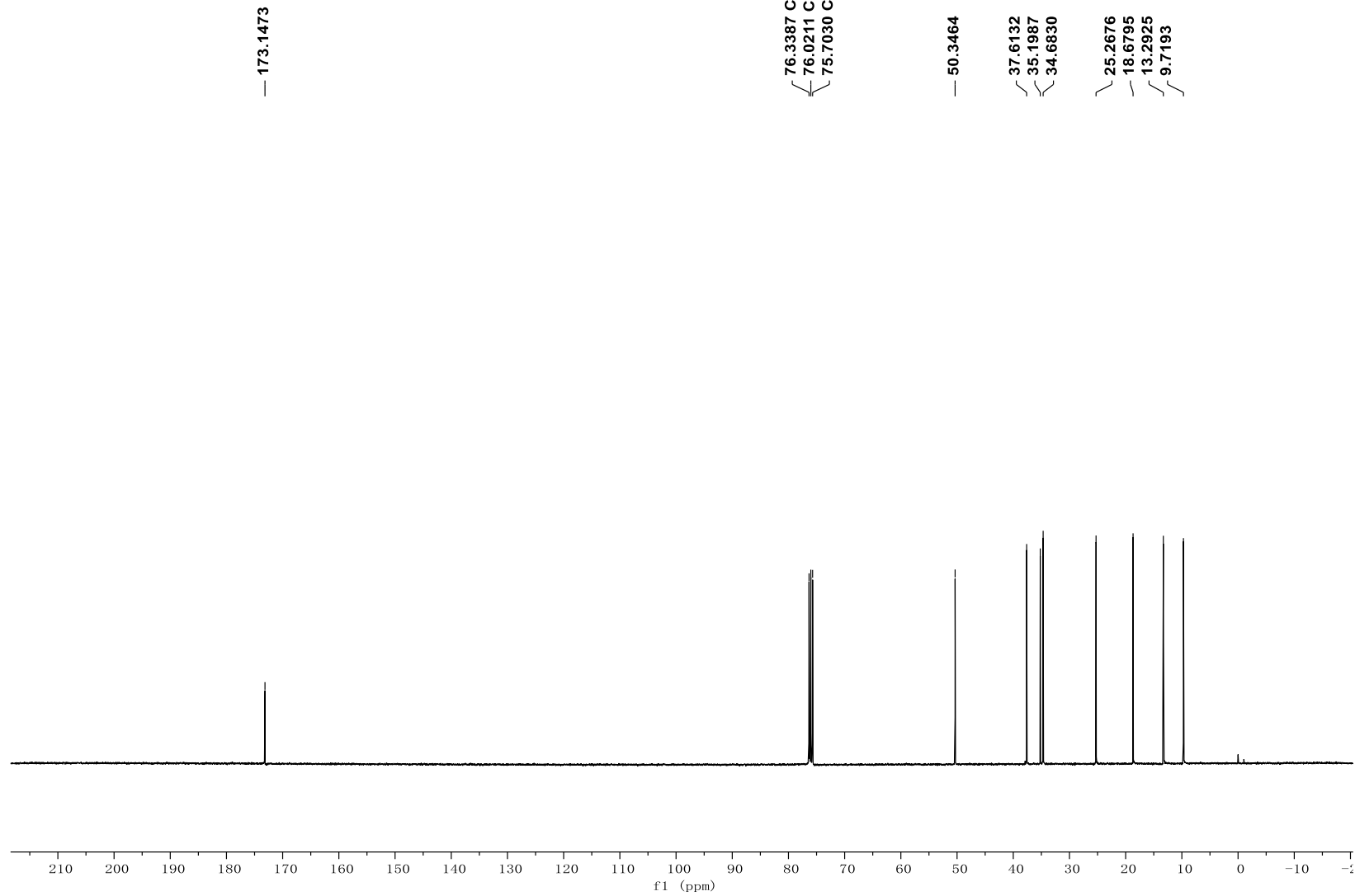


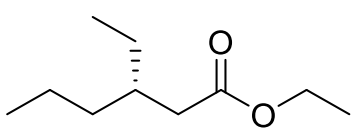

(25j)
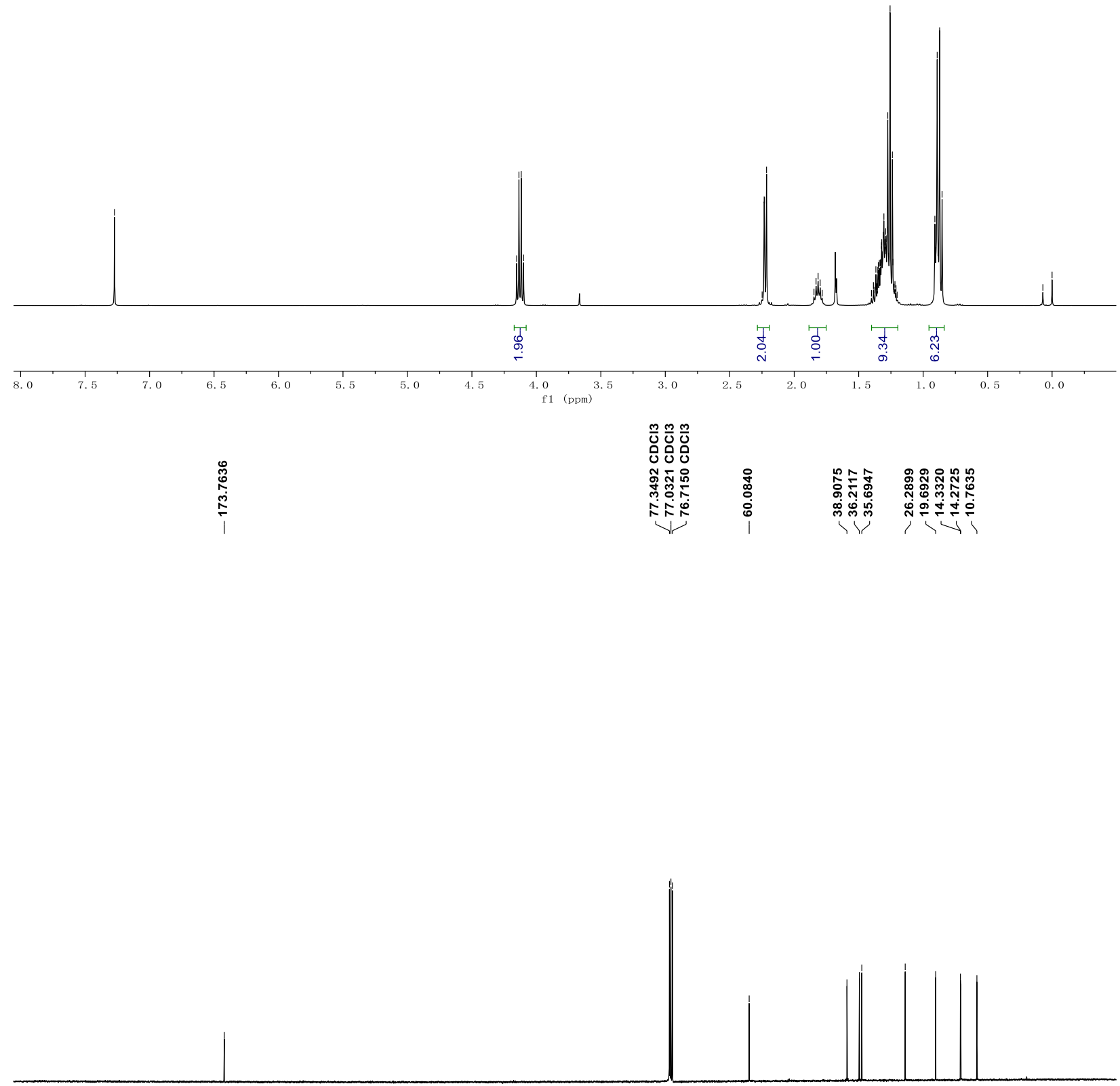

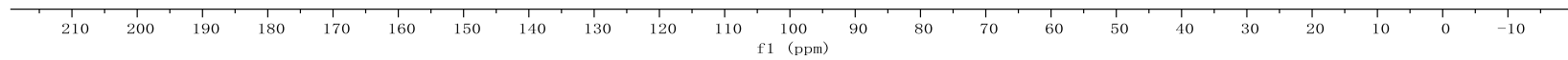



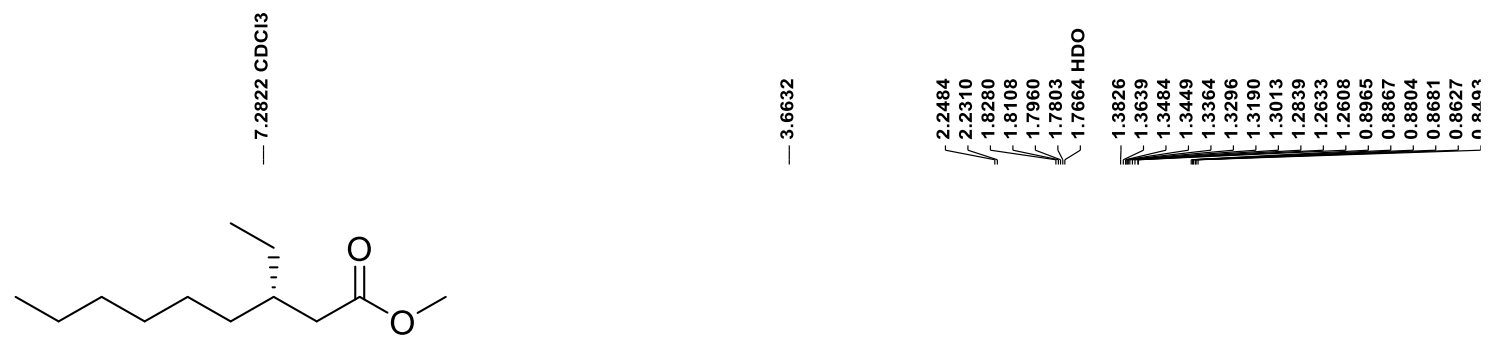

(25k)

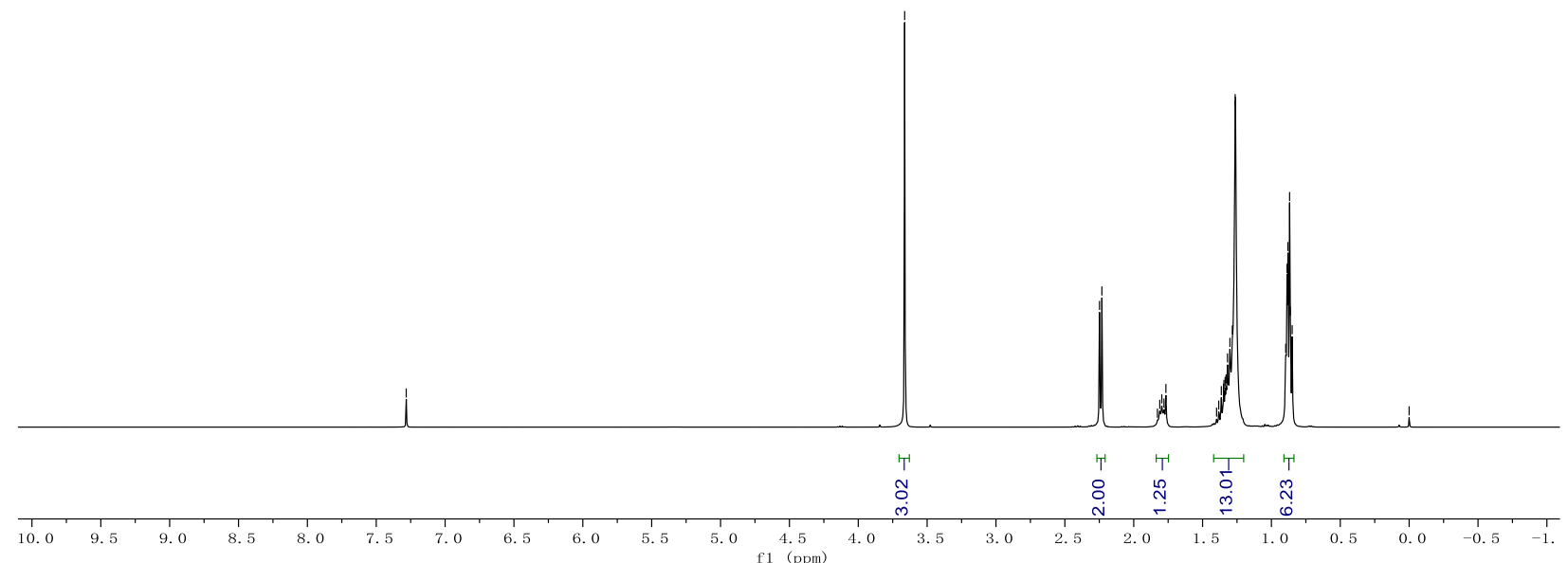

i

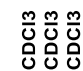

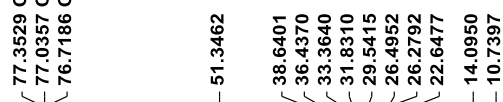
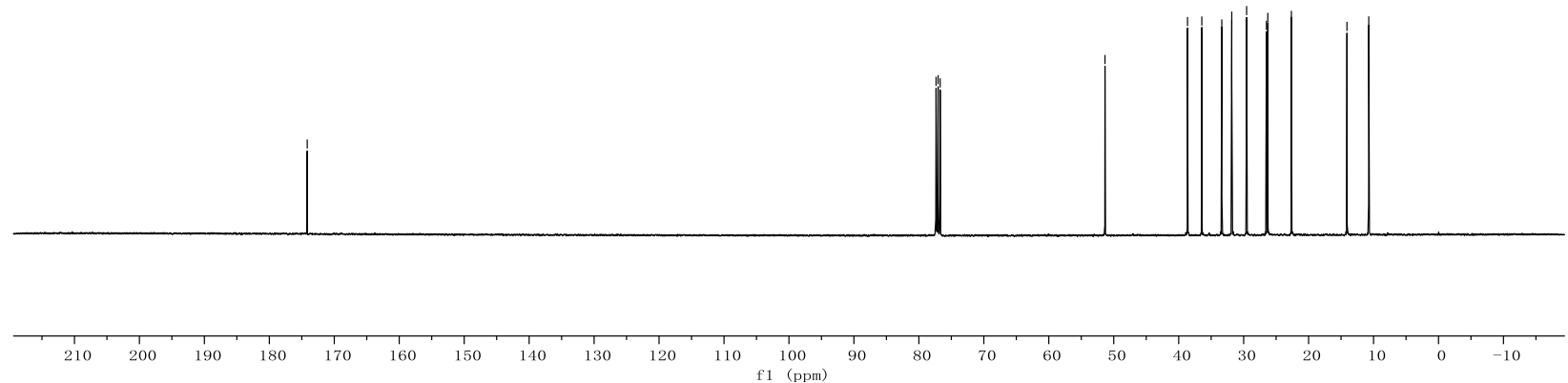


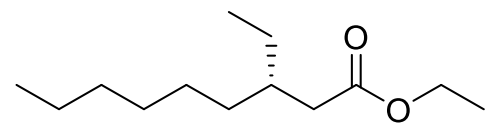

(25I)

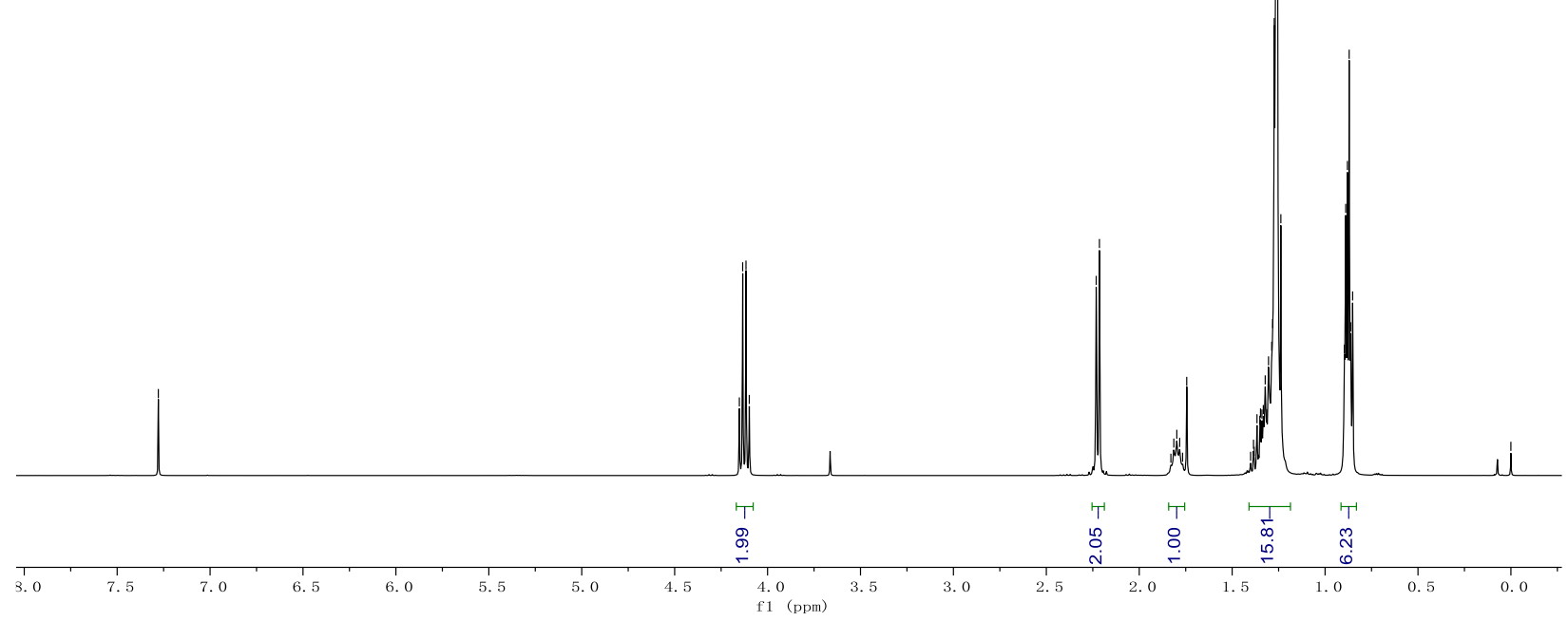

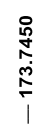

$\begin{array}{ll}\frac{m}{0} & \frac{m}{0} \\ 0 & 0 \\ 000 \\ 0 \\ 0\end{array}$

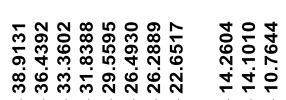

势
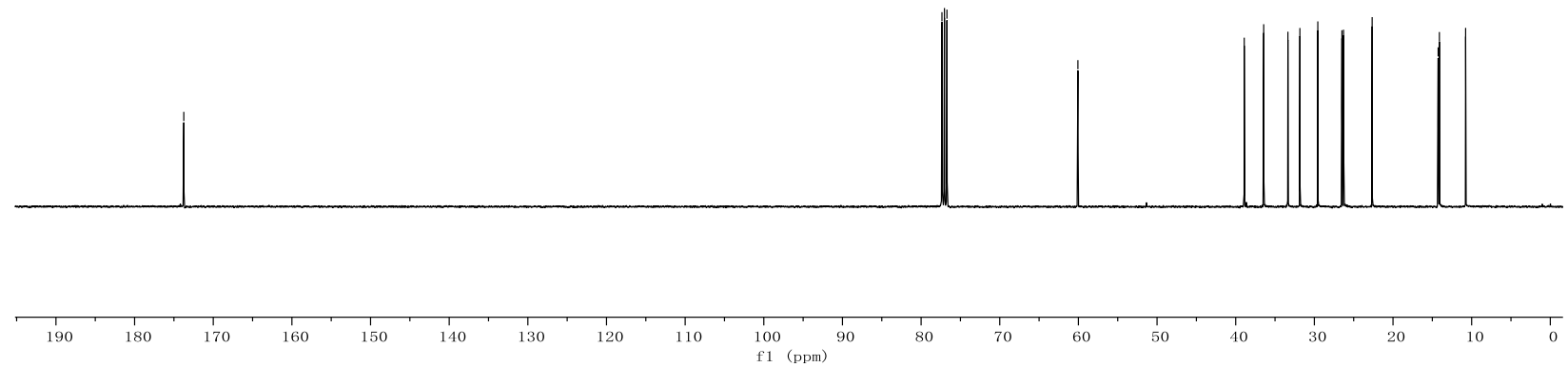


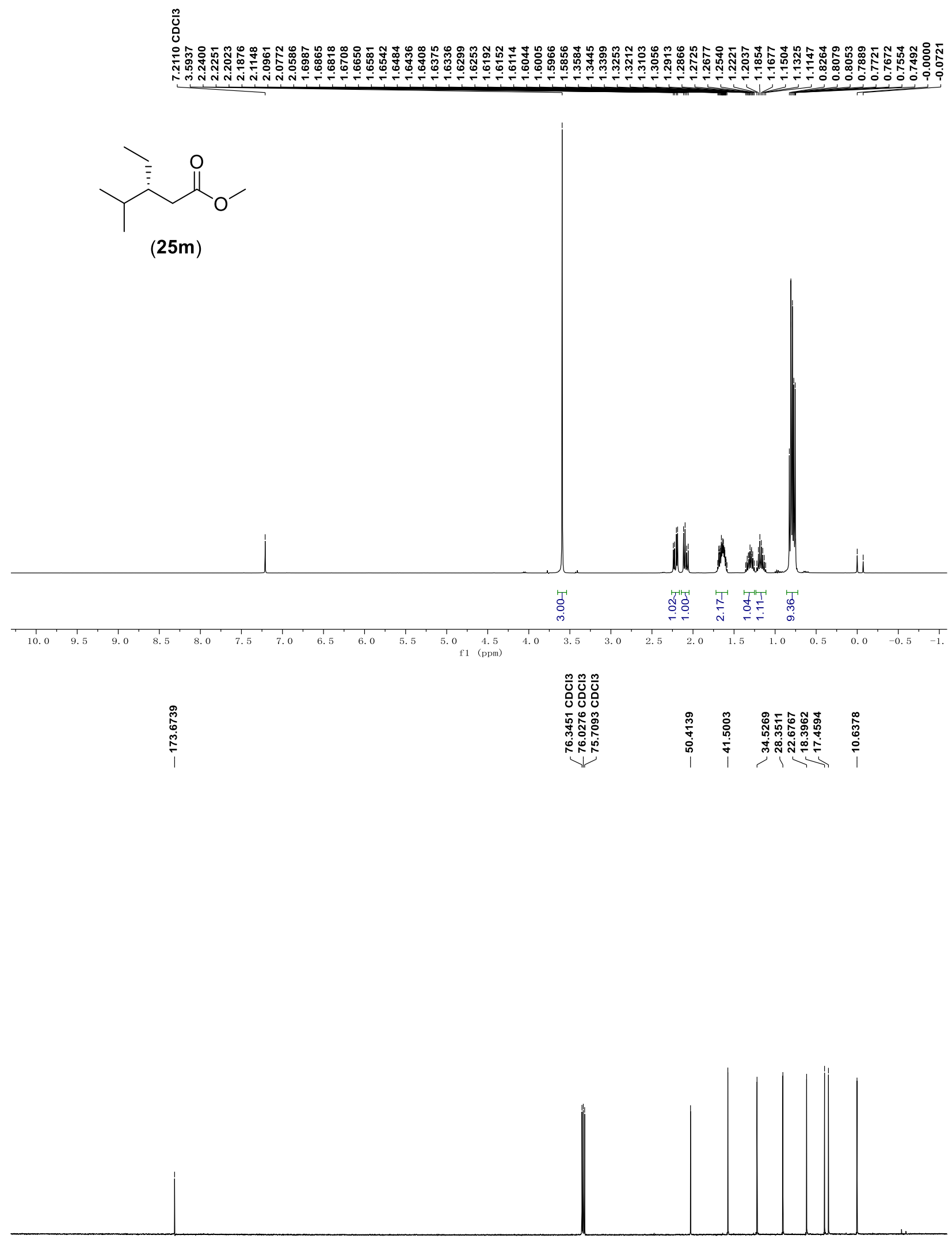

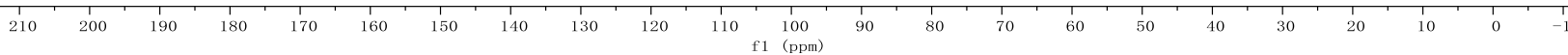




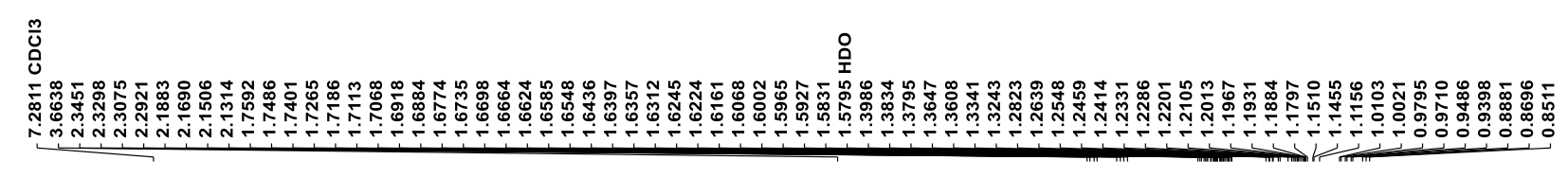<smiles>CC[C@H](CC(=O)OC)C1CCCCC1</smiles>

(25n)

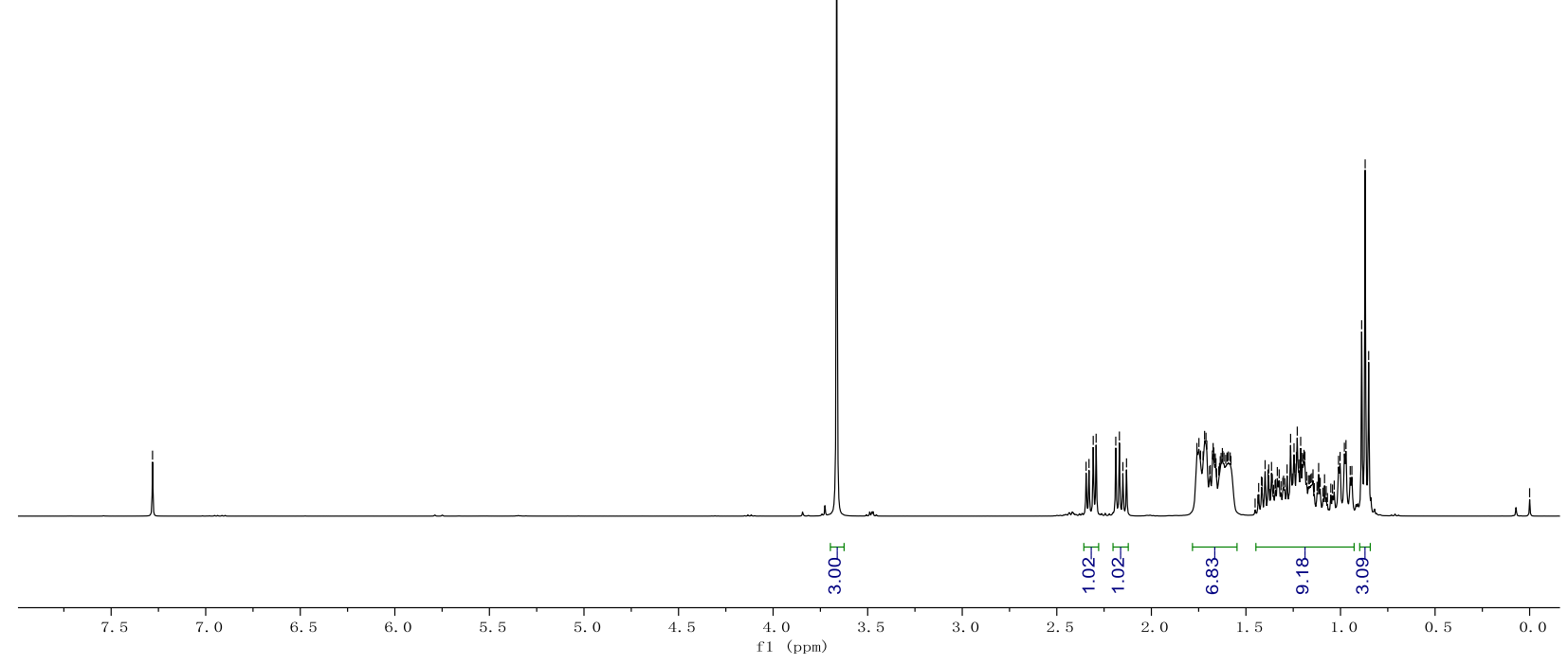

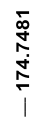

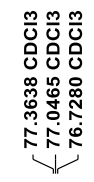

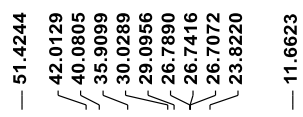
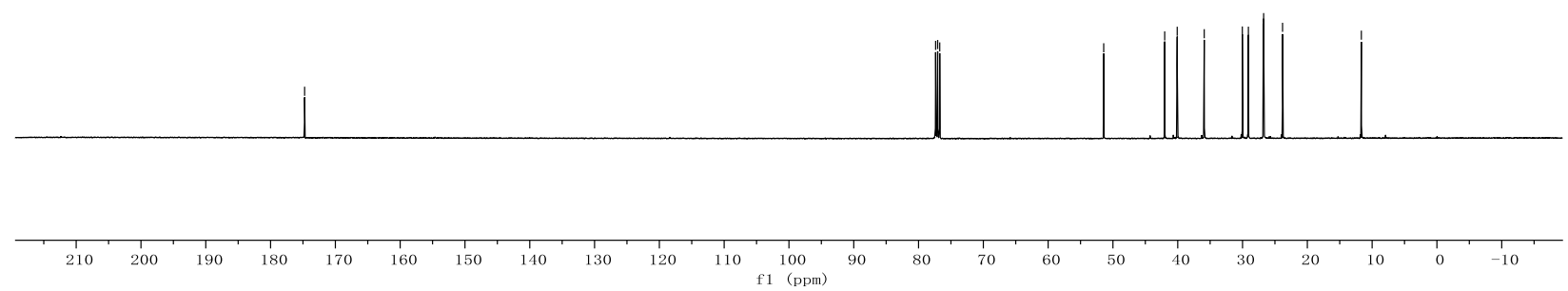

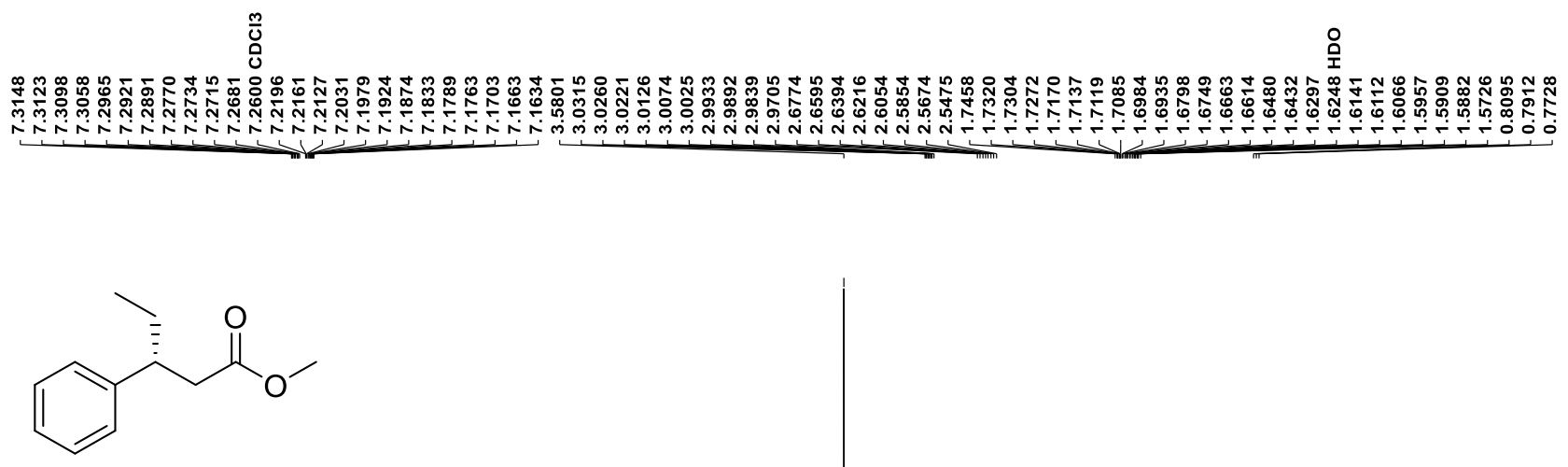

(250)

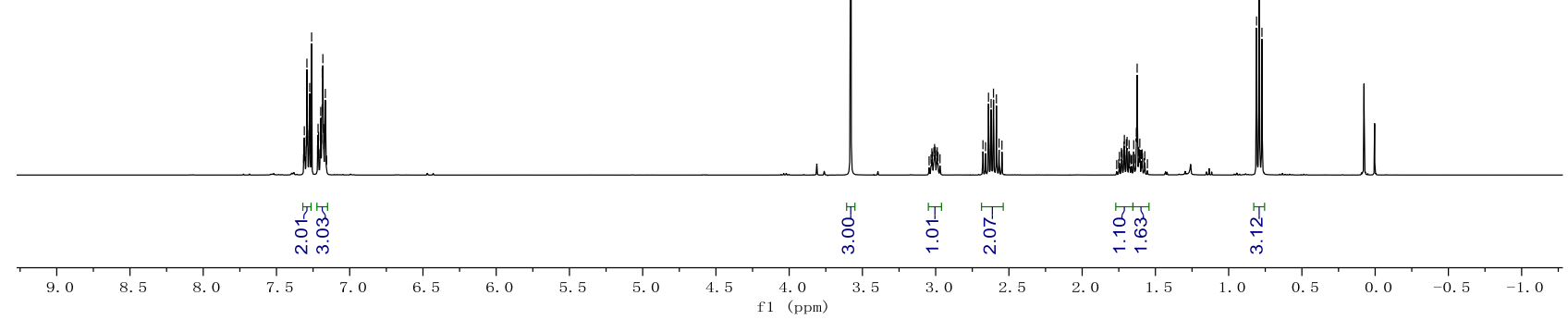

\begin{tabular}{|c|c|c|c|c|c|c|}
\hline 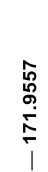 & 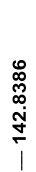 & 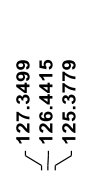 & 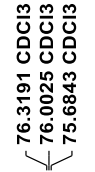 & $\begin{array}{l}\hat{y} \\
\text { ơ } \\
\dot{0} \\
1\end{array}$ & 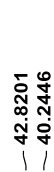 & 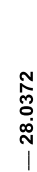 \\
\hline
\end{tabular}
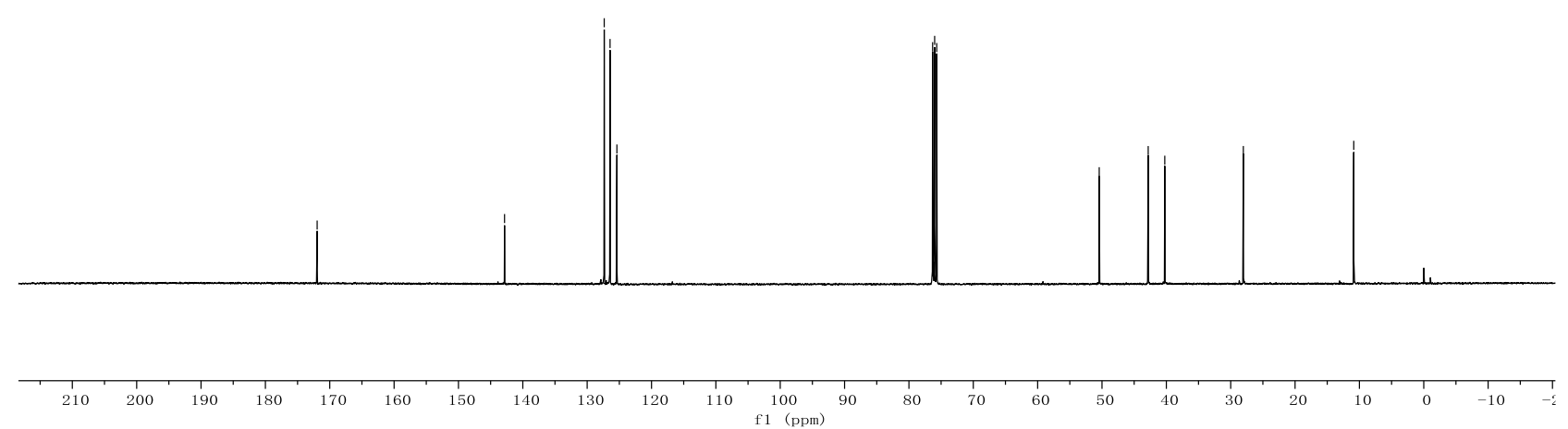


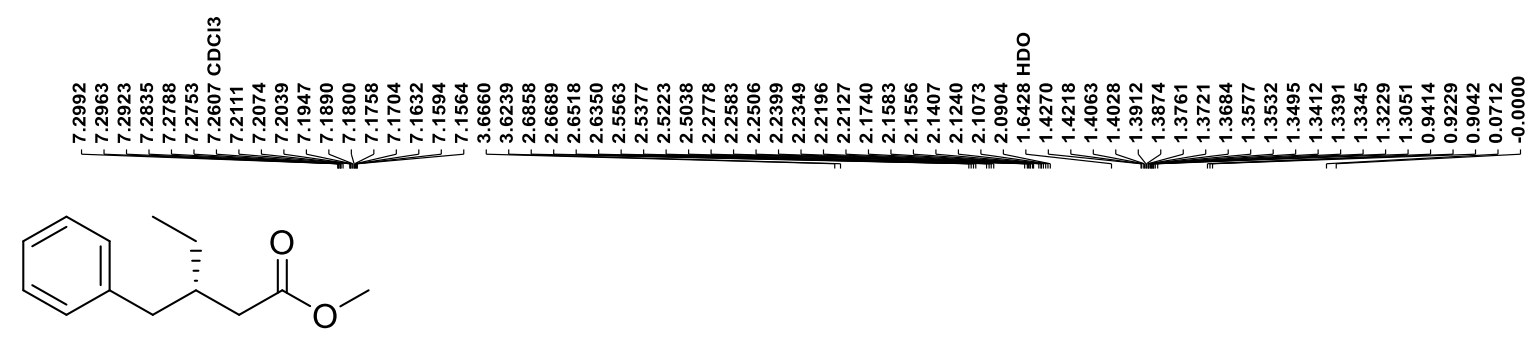

(25p)
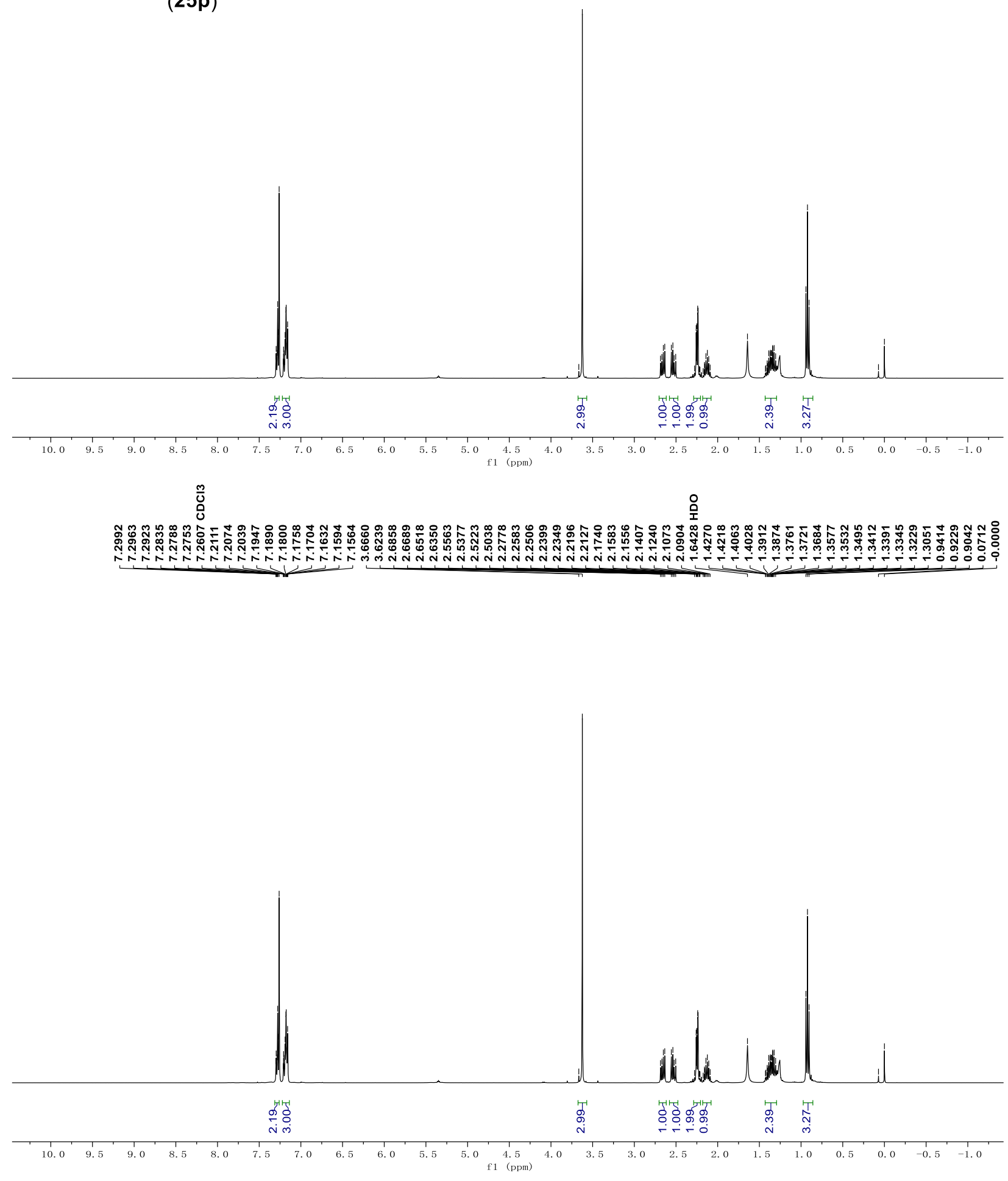


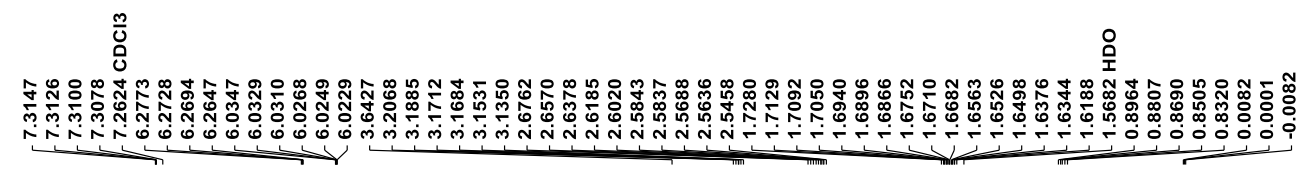<smiles>CC[C@@H](CC(=O)OC)c1ccco1</smiles>

(25q)

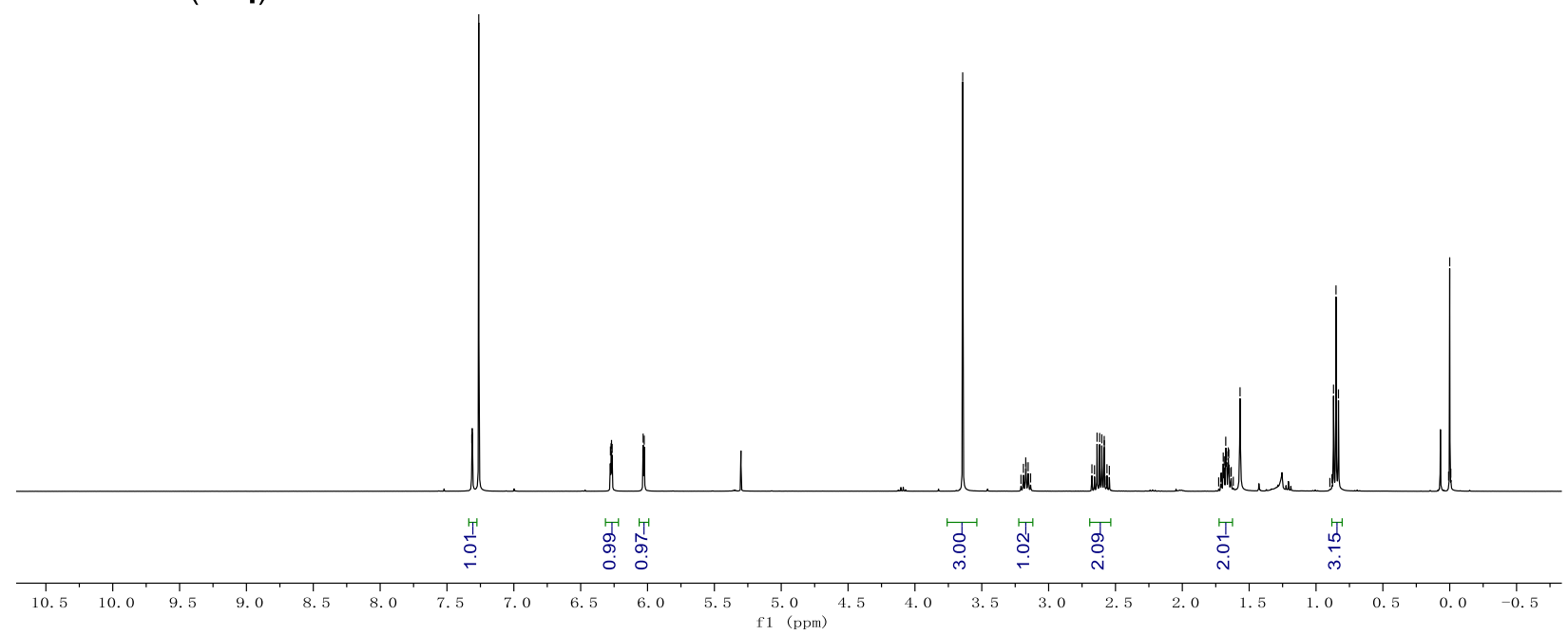

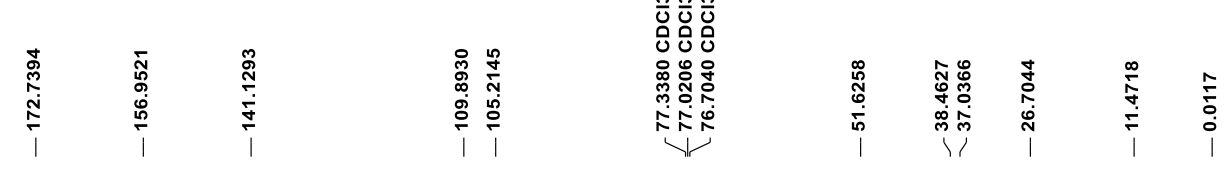

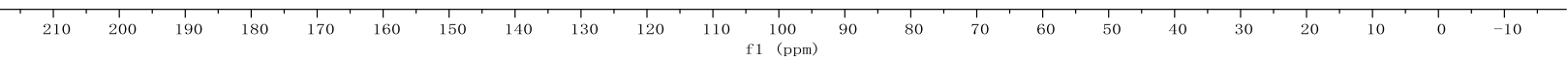


$\frac{m}{0}$

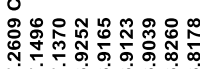

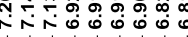

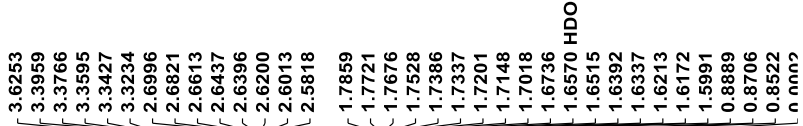

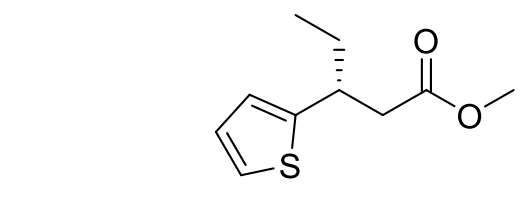

(25r)

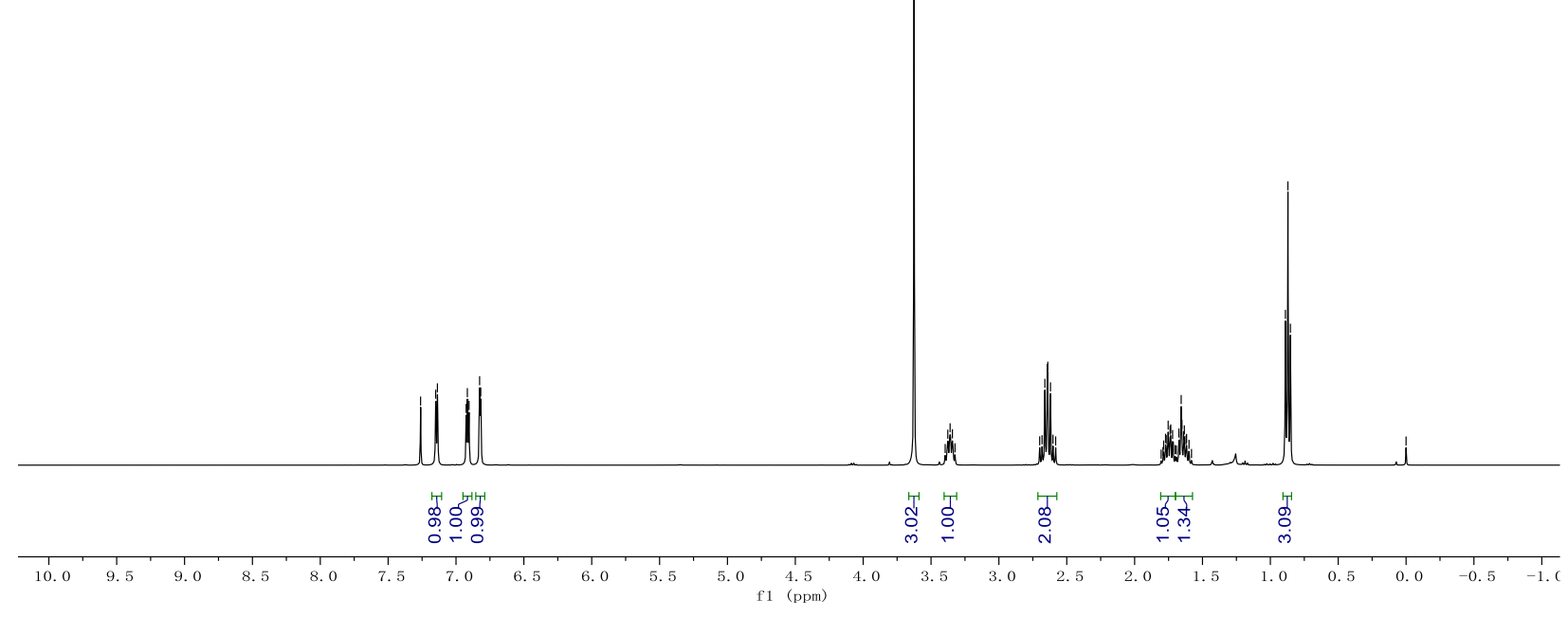

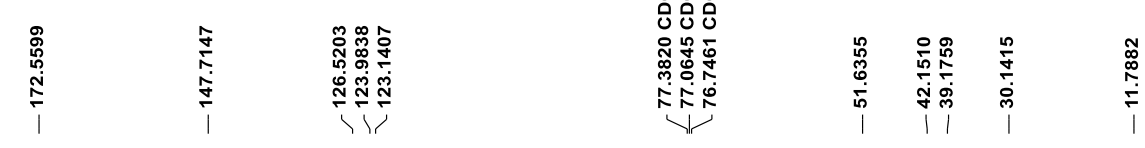
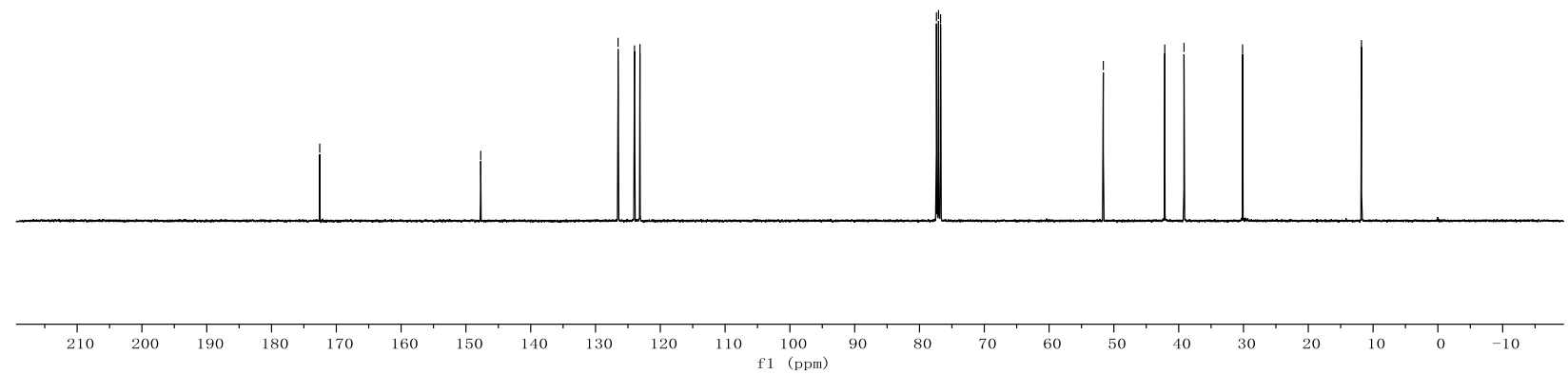


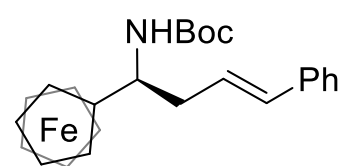

$\left(15 \mathbf{a}^{\prime}\right)$

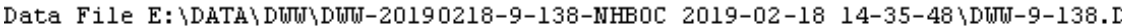
Sample Name: DUti-9-138-NHBoc-rac

\begin{tabular}{|c|c|c|c|}
\hline Acq. Operator & : SYSTEM & seq. Line : & 1 \\
\hline Acq. Instrument & : 1260 & Location : & 51 \\
\hline Injection Date & : 2/18/2019 2:37:23 PM & $\begin{array}{r}\text { Inj : } \\
\text { Inj Volume : }\end{array}$ & $\begin{array}{l}1 \\
0.00\end{array}$ \\
\hline
\end{tabular}

Acq. Method : E: \DATA \DIJ\DUJJ-20190218-9-138-NHBoc 2019-02-18 14-35-48 6-0DH-90-10-254NM1. $0 \mathrm{ML}-5 \mathrm{UL}--\mathrm{ALL}, \mathrm{M}$

Last changed : 2/18/2019 2:35:48 PM by SYSTEM

Analysis Method : E: \DATA \DाJ\DUJJ-20190218-9-138-NHBoc 2019-02-18 14-35-48 6-0DH-90-10-254NM1. OML-5UL--ALL.M (Sequence Method)

Last changed : 7/24/2020 8:41:03 AM by SYSTEM (modified after loading)

Additional Info : Peak (s) manually integrated

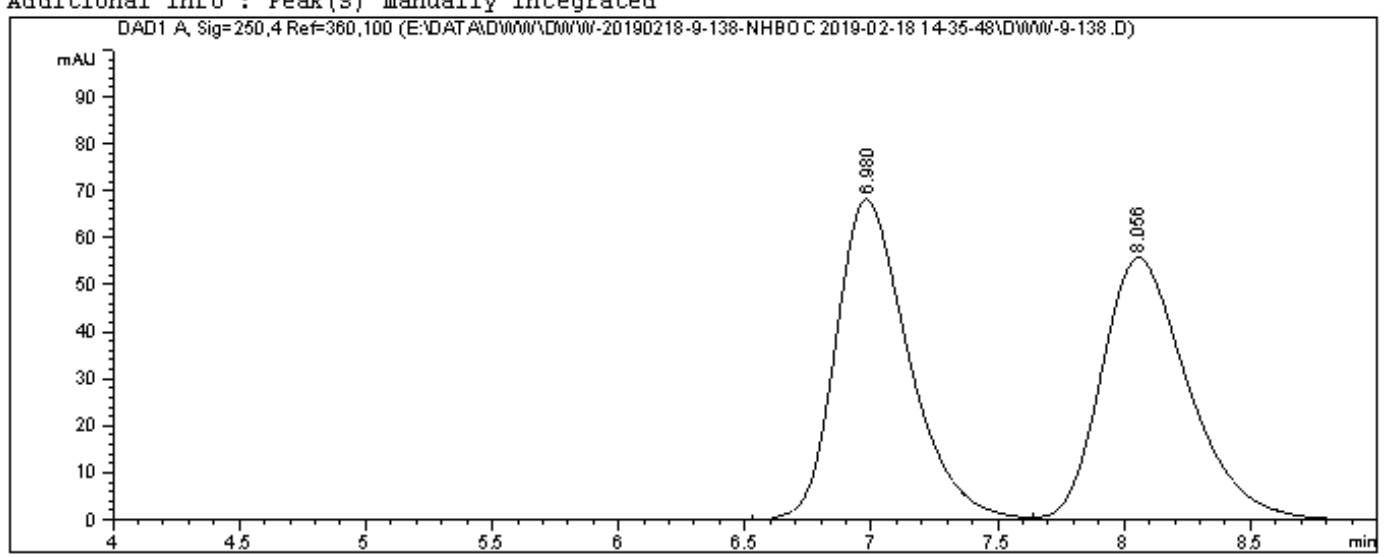

Area Percent Report

\begin{tabular}{|c|c|c|}
\hline Sorted BY & : & Signal \\
\hline Multiplier & : & 1.0000 \\
\hline Dilution & : & 1.0000 \\
\hline
\end{tabular}

Do not use Multiplier \& Dilution Factor with ISTDs

Signal 1: DADl A, Sig $=250,4$ Ref $=360,100$

\begin{tabular}{|c|c|c|c|c|c|c|}
\hline $\begin{array}{c}\text { Peak } \\
\#\end{array}$ & $\begin{array}{c}\text { RetTime } \\
\text { [min] }\end{array}$ & Type & $\begin{array}{l}\text { Width } \\
\text { [min] }\end{array}$ & $\begin{array}{c}\text { Area } \\
\text { [mAUt*s] }\end{array}$ & $\begin{array}{l}\text { Height } \\
\text { [mAU] }\end{array}$ & Area \\
\hline-- & $-1-$ & & ---1 & --------- & ------ & - \\
\hline 1 & 6.980 & $\mathrm{BV}$ & 0.3029 & 1381.60901 & 68.60628 & 50.6813 \\
\hline 2 & 8.056 & VB & 0.3427 & 1344.46497 & 56.07000 & 49.3187 \\
\hline Tota & : & & & 2726.07397 & 124.67627 & \\
\hline
\end{tabular}

*ネ* End of Report *** 


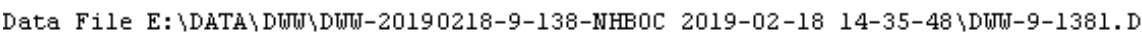
Sample Name: Duti-9-138-NHBoc-5

$\begin{array}{llrl}\text { Acq. Operator } & : \text { SYsTEM } & \text { Seq. Line }: & 2 \\ \text { Acq. Instrument }: 1260 & \text { Location }: & 52 \\ \text { Injection Date }: 2 / 18 / 2019 & 2: 53: 50 \mathrm{PM} & \text { Inj }: & 1 \\ & & \text { Inj volume : } 10.000 \mu \mathrm{l}\end{array}$

Acq. Method : E: \DATA \DाJ\DUJJ-20190218-9-138-NHBoc 2019-02-18 14-35-48 6-0DH-90-10-254NM1. OML-5UL--ALL.M

Last changed : 2/18/2019 2:35:48 PM by SYSTEM

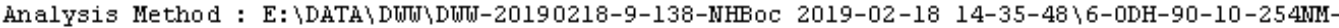
1. OML-5UL--ALL.M (Sequence Method)

Last changed : 7/24/2020 8:44:12 AM by SYSTEM

(modified after loading)

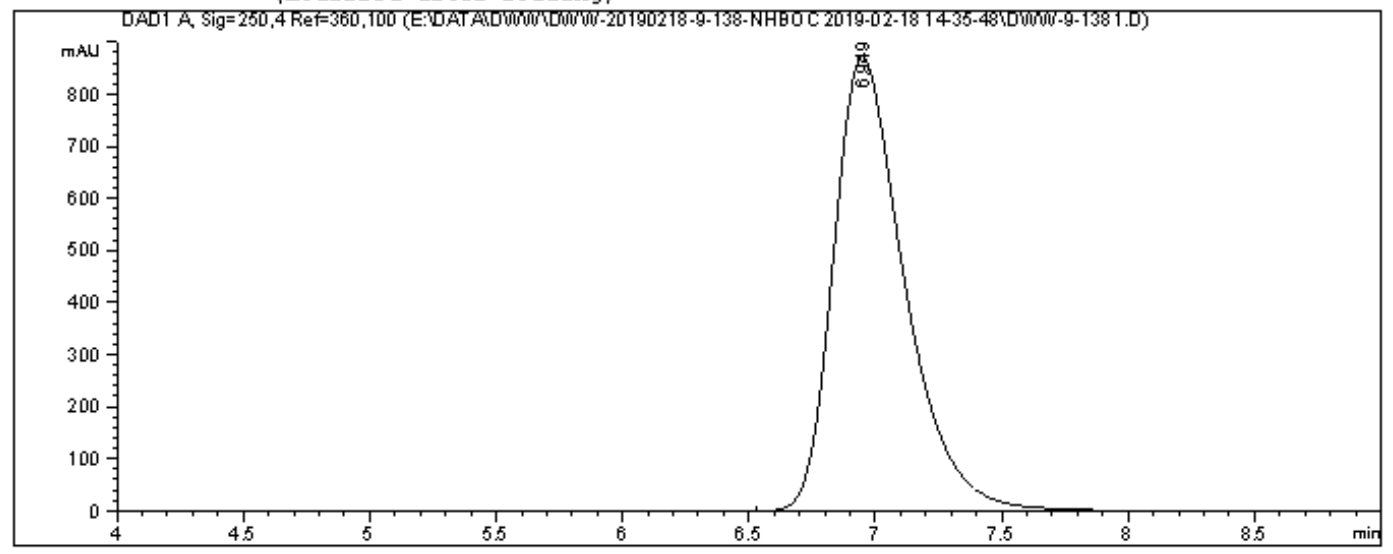

Area Percent Report

\begin{tabular}{|c|c|c|}
\hline Sorted By & : & signal \\
\hline Multiplier & : & 1.0000 \\
\hline Dilution & : & 1.0000 \\
\hline
\end{tabular}

Do not use Multiplier \& Dilution Factor with IsTDs

Signal 1: DẢDl Aे, Sig=250, 4 Ref $=360,100$

Peak RetTime Type width Area Height Area

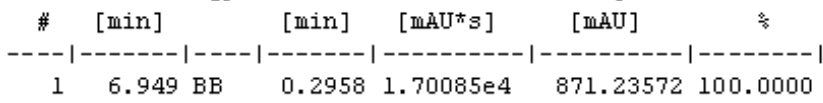

Totals : $\quad 1.70085 \mathrm{e} 4 \quad 871.23572$

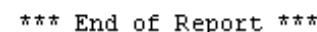


<smiles>O=C(O)[NH2+][C@H](C/C=C/c1ccc2ccccc2c1)c1ccccc1</smiles>

$\left(15 b^{\prime}\right)$

Data File E: \DATA $\backslash$ LYN $\backslash$ LN-4-14 2019-11-20 12-19-35\LYN-4-141.D Sample Name: LYN-4-14-RAC
Acq. Operator : SYSTEM
Ac q. Instrument : 1260
Seq. Line : 2
Location : 1
Injection Date : 11/20/2019 12:31:59 PM
Inj : 1
Inj Volume : $10.000 \mu \mathrm{l}$

Ácq. Method : E: \DATA $\backslash$ LYN $\backslash$ LYN-4-14 2019-11-20 12-19-35 AADH-90-10-40min-254nm-1ML-10ul-RAC . $\mathrm{M}$

Last changed : 11/20/2019 12:19:36 PM by SYSTEM

Analysis Method : E: \DATA LYN LYN-4-14 2019-11-20 12-19-35 ÄDH-90-10-40min-254nm-1ML-10ul-RAC .M (Sequence Method)

Last changed : 7/24/2020 10:08:42 AM by SYSTEM

(modified after loading)

Additional Info: Peak (s) manually integrated

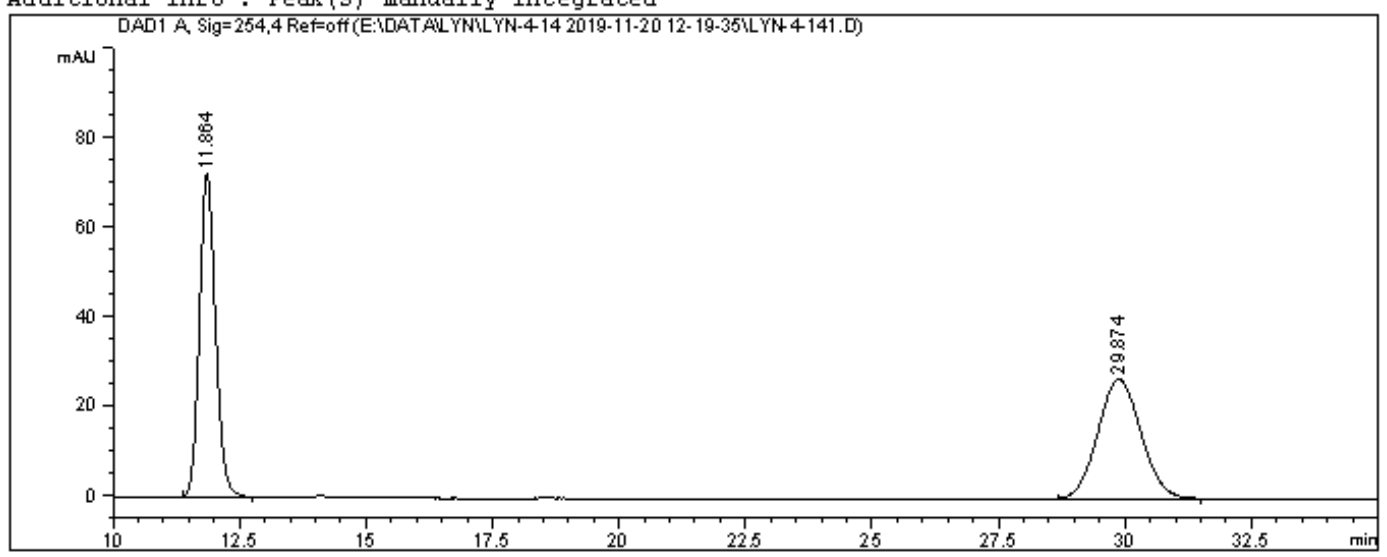

\section{Area Percent Report}

\begin{tabular}{|c|c|c|}
\hline Sorted By & : & Signal \\
\hline Multiplier & : & 1.0000 \\
\hline Dilution & : & 1.0000 \\
\hline
\end{tabular}

Do not use Multiplier \& Dilution Factor with ISTDs

Signal 1: DADl मे, Sig=254,4 Ref=off

\begin{tabular}{|c|c|c|c|c|c|}
\hline $\begin{array}{c}\text { Peak } \\
\#\end{array}$ & $\begin{array}{l}\text { RetTime Type } \\
\text { [min] }\end{array}$ & $\begin{array}{l}\text { Width } \\
\text { [min] }\end{array}$ & $\begin{array}{c}\text { Area } \\
{\left[\mathrm{mAU} \mathrm{U}^{*} \mathrm{~S}\right]}\end{array}$ & $\begin{array}{l}\text { Height } \\
\text { [mAU] }\end{array}$ & $\begin{array}{c}\text { Area } \\
\stackrel{5}{*}\end{array}$ \\
\hline-- & & 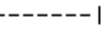 & ---------- & ------- & 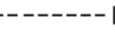 \\
\hline 1 & $11.864 \mathrm{BB}$ & 0.3387 & 1605.21179 & 72.10893 & 50.4605 \\
\hline 2 & $29.874 \mathrm{BB}$ & 0.6976 & 1575.91345 & 26.62316 & 49.5395 \\
\hline t & : & & 3181.12524 & 98.73209 & \\
\hline
\end{tabular}

ネネネ End of Report ネネ゙ 
Data File E: \DATA LYN LYN-4-14 2019-11-20 12-19-35\LWN-4-142.D

Sample Name: LYN-4-14-GH

Acq. Operator : SYSTEM

Acq. Instrument : 1260

Injection Date : 11/20/2019 1:13:29 PM

Ac q. Method

Last changed .M

Analysis Method : E: \DATA $\backslash$ LYN LYN-4-14 2019-11-20 12-19-35\ADH-90-10-40min-254nm-1ML-10ul-RAC .M (Sequence Method)

Last changed : 7/24/2020 10:12:01 AM by SYSTEM

(modified after loading)

Additional Info : Peak (s) manually integrated

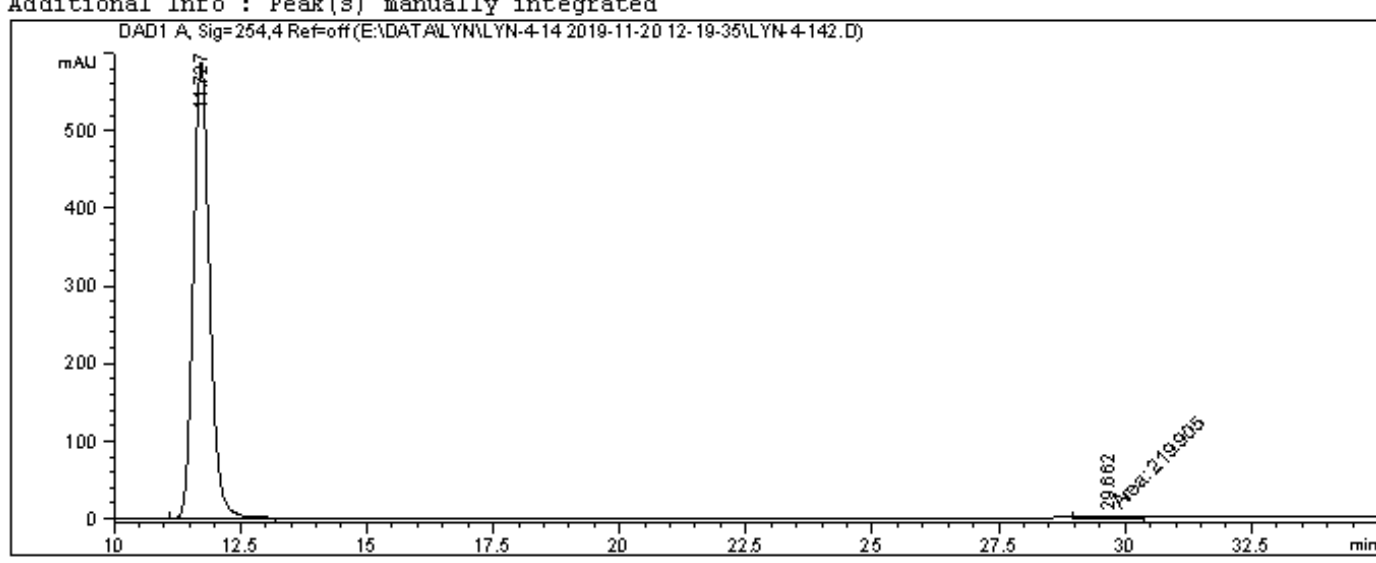

\section{Àrea Percent Report}

\begin{tabular}{|c|c|c|}
\hline Sorted By & : & Signal \\
\hline Multiplier & : & 1.0000 \\
\hline Dilution & : & 1.0000 \\
\hline
\end{tabular}

Do not use Multiplier \& Dilution Factor with ISTDs

Signal 1: DADD Â, Sig=254,4 Ref=off

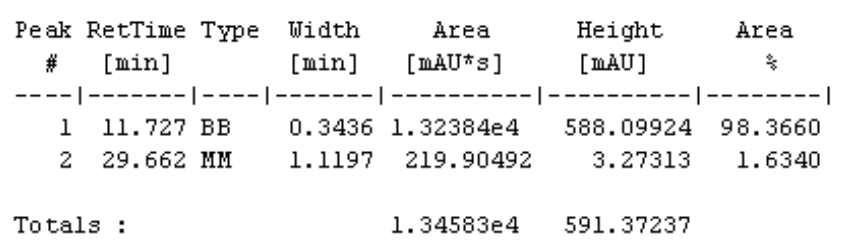

ネネส End of Report ネネ 
<smiles>Cc1ccc(/C=C/C[C@H](NC(=O)OCc2ccccc2)c2ccccc2)cc1</smiles>

$\left(15 c^{\prime}\right)$

Data File E: \DATA LYN LYN-4-8285 2019-12-19 16-51-14\LYN-4-82851.D Sample Name: LYN-4-82-RAC

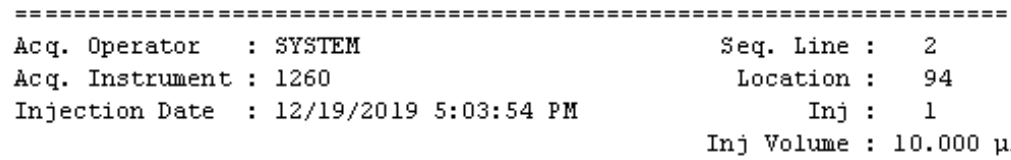

Acq. Method : E: \DATA $\backslash$ LYN $\backslash$ LYN-4-8285 2019-12-19 16-51-14\ADH-90-10-40min-254nm-1ML-10ulRAC.M

Last changed : 12/19/2019 4:51:14 PM by SYSTEM

Analysis Method : E: \DATA \LN\LYN-4-8285 2019-12-19 16-51-14\ADH-90-10-40min-254nm-1ML-10ulRAC.M (SEequence Method)

Last changed : 7/24/2020 9:07:24 AM by SYSTEM (modified after loading)

Additional Info : Peak (s) manually integrated

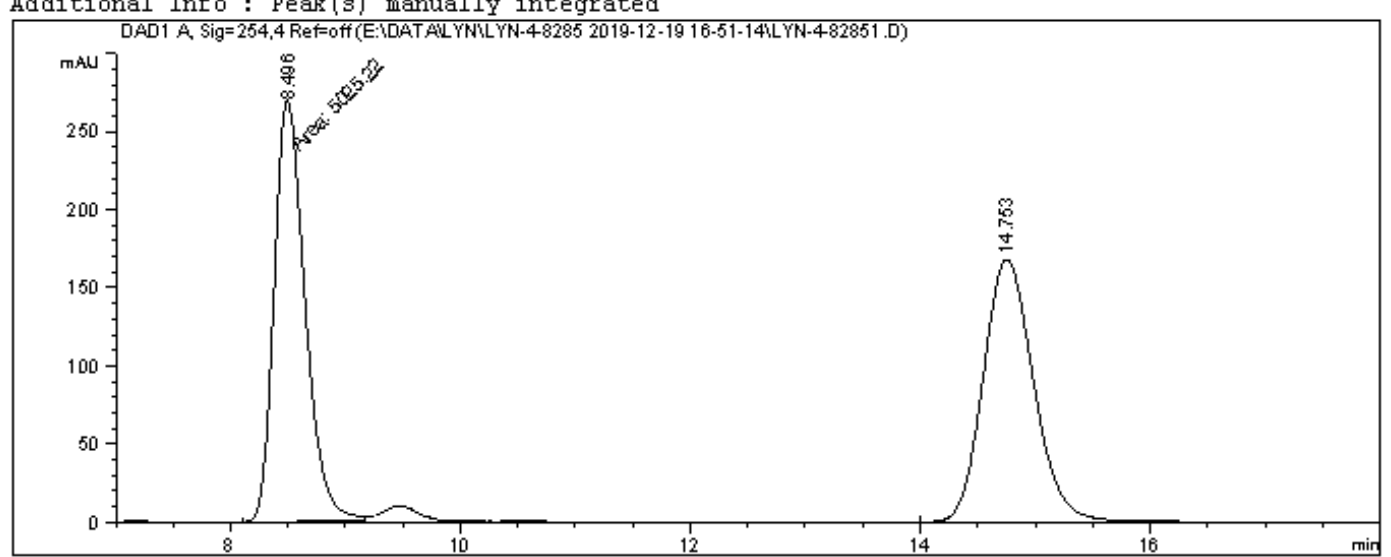

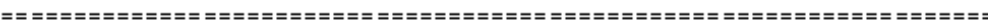

Area Percent Report

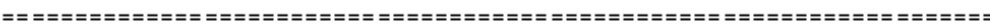

$\begin{array}{lll}\text { Sorted BY } & : & \text { Signal } \\ \text { Multiplier } & : & 1.0000\end{array}$

Dilution $\quad$ : 1.0000

Do not use Multiplier \& Dilution Factor with ISTDs

Signal 1: DADl A, Sig=254, 4 Ref=off

\begin{tabular}{|c|c|c|c|c|c|}
\hline $\begin{array}{c}\text { Peak } \\
\#\end{array}$ & $\begin{array}{l}\text { RetTime Type } \\
\text { [min] }\end{array}$ & $\begin{array}{l}\text { Width } \\
\text { [min] }\end{array}$ & $\begin{array}{c}\text { Arrea } \\
{\left[\text { mA } U^{*} \text { S }\right]}\end{array}$ & $\begin{array}{l}\text { Height } \\
\text { [MAU] }\end{array}$ & $\begin{array}{c}\text { Area } \\
\stackrel{4}{*}\end{array}$ \\
\hline & & & & & \\
\hline 1 & $5 \mathrm{MF}$ & & 5025 . & 30341 & 388 \\
\hline 2 & 4. $753 \mathrm{BB}$ & 40 & 5098.35693 & 168.02318 & 50.3612 \\
\hline
\end{tabular}

Totals : $\quad 1.01236 \mathrm{e} 4 \quad 437.32658$

** End of Report $* * *$ 
Data File E: \DATA \LM LYN-4-8285 2019-12-19 16-51-14\LYN-4-82852.D

Sample Name: LYN-4-82-GH

Acq. Operator : SYSTEM

Acq. Instrument : 1260

Injection Date : 12/19/2019 5:45:19 PM

Ac q. Method seq. Line : 3

Location : 95

Inj : 1

Inj Volume : $10.000 \mu l$

: E: \DATA $\backslash$ LYN $\backslash$ LYN-4-8285 2019-12-19 16-51-14 1 ADH-90-10-40m in-254nm-1ML-10ulRAC.M

Last changed : 12/19/2019 4:51:14 PM by SYSTEM

Analysis Method : E: \DATA $\backslash$ LN\LYN-4-8285 2019-12-19 16-51-14\ADH-90-10-40min-254nm-1ML-10ulRAC.M (Sequence Method)

Last changed : 7/24/2020 9:12:27 AM by SYSTEM

(modified after loading)

Additional Info : Peak (s) manually integrated

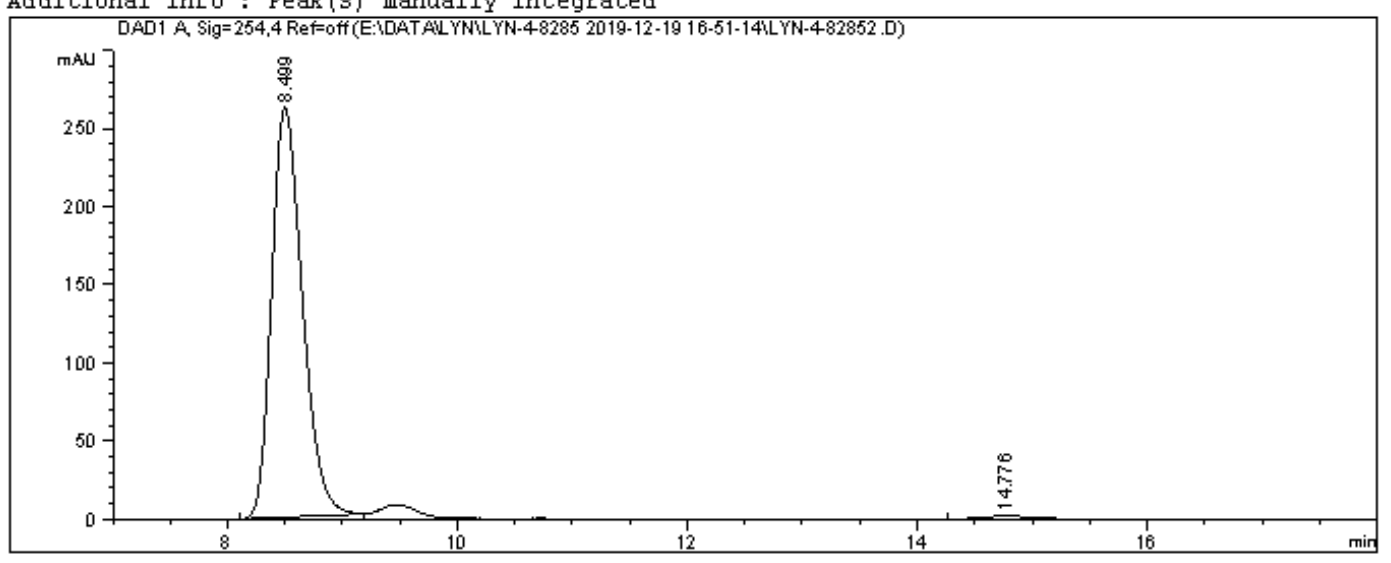

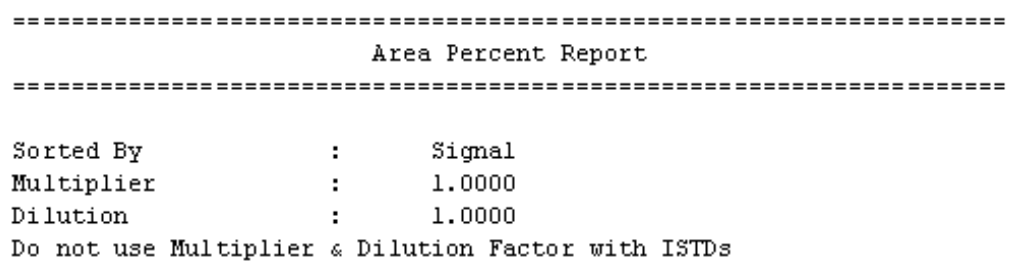

Do not use Multiplier \& Dilution Factor with IsTDs

Signal 1: DADD A, Sig=254,4 Ref=off

\begin{tabular}{|c|c|c|c|c|c|c|}
\hline $\begin{array}{c}\text { Peak } \\
\#\end{array}$ & $\begin{array}{c}\text { RetTime } \\
\text { [min] }\end{array}$ & Type & $\begin{array}{l}\text { Width } \\
\text { [min] }\end{array}$ & $\begin{array}{c}\text { Area } \\
\text { [mAUt*s] }\end{array}$ & $\begin{array}{l}\text { Height } \\
\text { [mAU] }\end{array}$ & $\begin{array}{c}\text { Area } \\
\stackrel{5}{*}\end{array}$ \\
\hline & & & 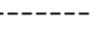 & -- & - & --- \\
\hline 1 & 8 & $8 B$ & 884 & 4853.81885 & 261.64490 & 98.7705 \\
\hline 2 & 14.776 & $\mathrm{BB}$ & 0.3311 & 60.41918 & 2.14811 & 1.2295 \\
\hline
\end{tabular}

Totals : $\quad 4914.23803 \quad 263.79301$ 
<smiles>NC(C/C=C/c1cc(C(=O)OC(F)(F)F)cc(C(F)(F)F)c1)c1ccccc1</smiles>

$\left(15 d^{\prime}\right)$

Data File E: \DATA $\backslash$ LYN $\backslash$ WCS-2-35 2019-12-05 22-40-08\LYN-4-19.D Sample Name: LYN-4-19-RAC

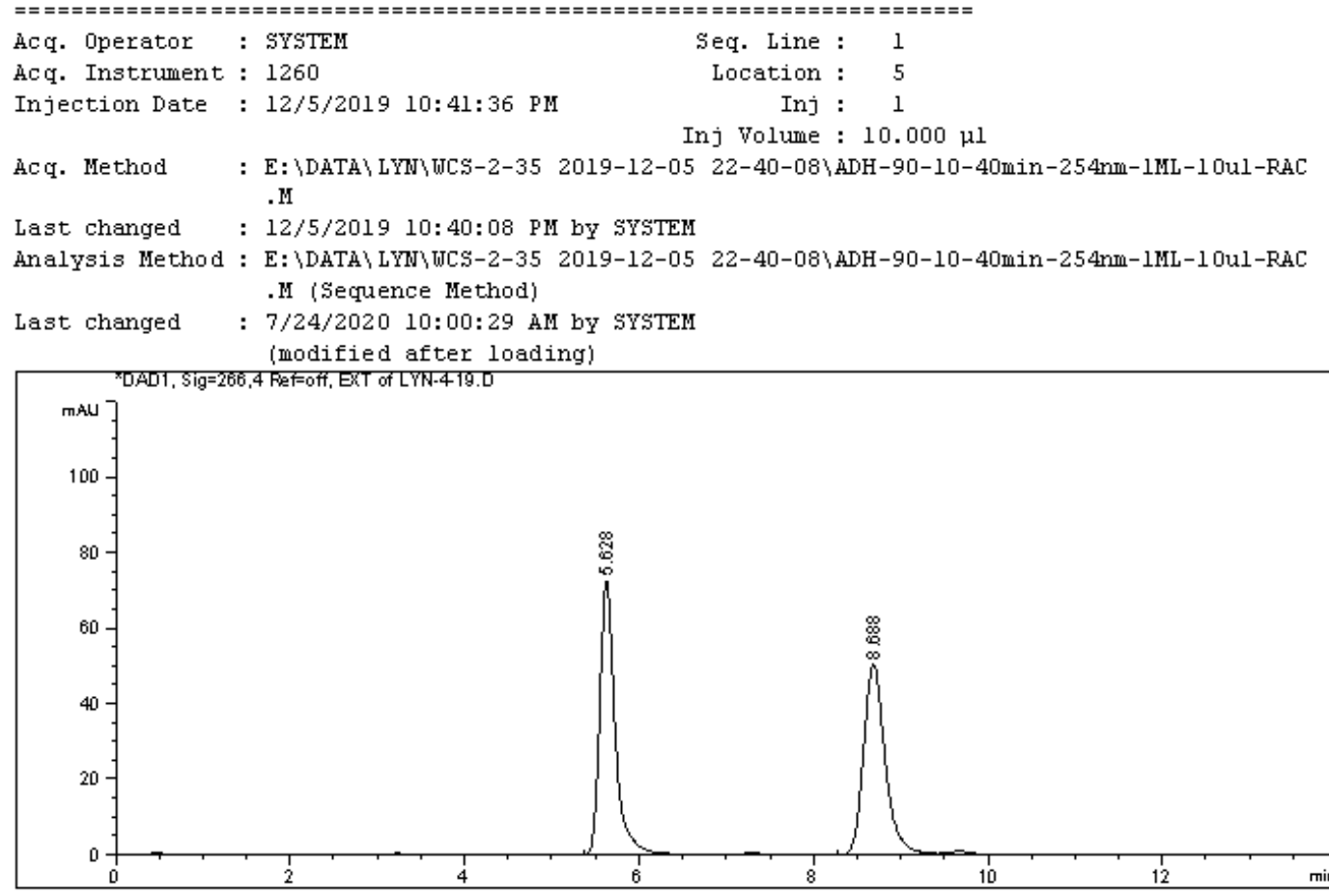

\section{Area Percent Report}

\begin{tabular}{|c|c|c|}
\hline Sorted By & : & Signal \\
\hline Multiplier & : & 1.0000 \\
\hline Dilution & : & 1.0000 \\
\hline
\end{tabular}

Do not use Multiplier \& Dilution Factor with ISTDs

Signal 1: DADl, Sig=266,4 Ref=off, EXT

signal has been modified after loading from rawdata file!

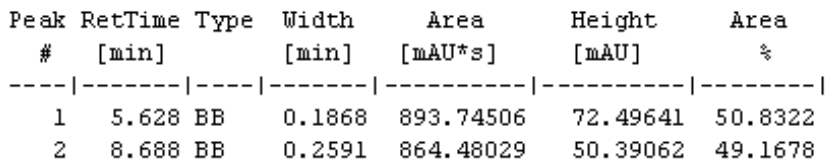

Totals :

$1758.22534 \quad 122.88703$ 
Data File E: \DATA LYN $\backslash$ UCS-2-35 2019-12-05 22-40-08\LWN-4-191.D

Sample Name: LYN-4-20-GH

\begin{tabular}{|c|c|c|c|}
\hline Acq. Operator & : SYSTEM & seq. Line : & 2 \\
\hline Acq. Instrument & : 1260 & Location : & 6 \\
\hline Injection Date & : 12/5/2019 11:23:09 PM & Inj : & 1 \\
\hline
\end{tabular}

Acq. Method : E: \DATA $\backslash$ LYN $\backslash$ WCS-2-35 2019-12-05 22-40-08\ADH-90-10-40min-254nm-1ML-10ul-RAC .M

Last changed : 12/5/2019 10:40:08 PM by SYSTEM

Analysis Method : E: \DATA LYN \WCS-2-35 2019-12-05 22-40-08\ADH-90-10-40min-254nm-1ML-10ul-RAC .M (Sequence Method)

Last changed : 7/24/2020 10:04:16 AM by SYSTEM

(modified after loading)

Additional Info : Peak (s) manually integrated

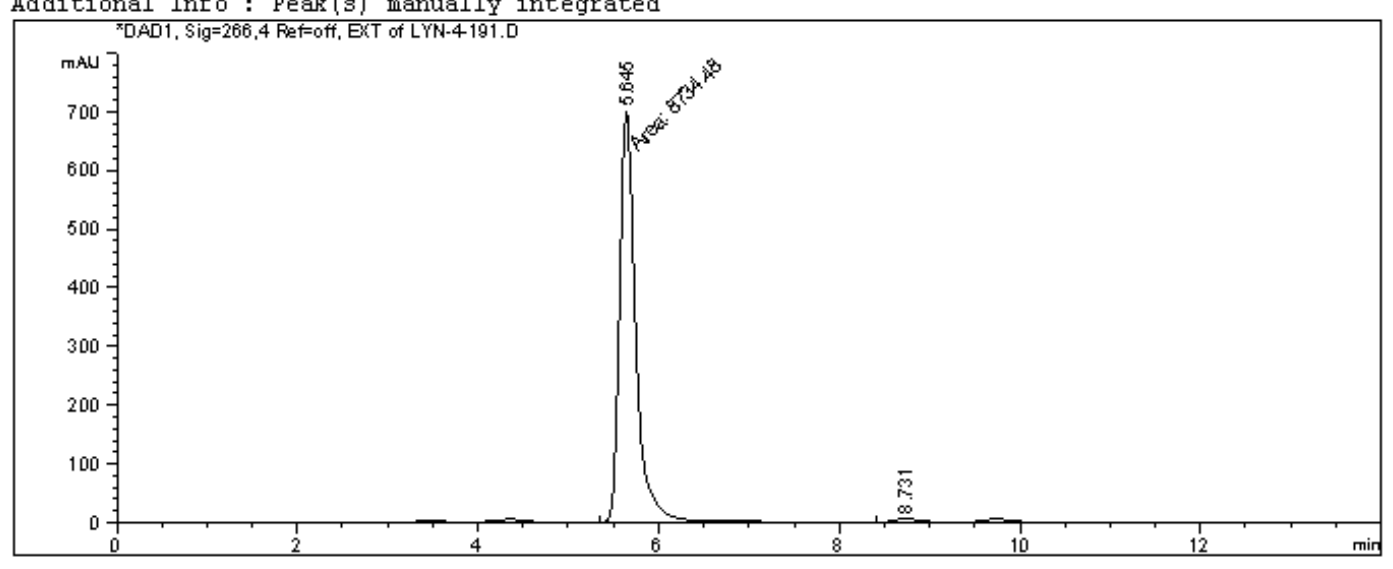

\section{Area Percent Report}

\begin{tabular}{|c|c|c|}
\hline Sorted BY & : & Signal \\
\hline Multiplier & : & 1.0000 \\
\hline Dilution & : & 1.0000 \\
\hline
\end{tabular}

Do not use Multiplier \& Dilution Factor with ISTDs

Signal 1: DADl, Sig=266,4 Ref=off, EXT

signal has been modified after loading from rawdata file!

\begin{tabular}{|c|c|c|c|c|c|c|}
\hline $\begin{array}{c}\text { Peak } \\
\#\end{array}$ & $\begin{array}{c}\text { RetTime } \\
\text { [min] }\end{array}$ & Type & $\begin{array}{l}\text { Width } \\
\text { [min] }\end{array}$ & $\begin{array}{c}\text { Area } \\
{[\text { [mÁU }}\end{array}$ & $\begin{array}{l}\text { Height } \\
\text { [min] }\end{array}$ & $\begin{array}{c}\text { Area } \\
\stackrel{5}{*}\end{array}$ \\
\hline & & & & & & \\
\hline & & & & 8734 & 699. & 98. \\
\hline & 8. & & 100 & 111.74388 & 6.45285 & 1.2632 \\
\hline
\end{tabular}

Totals : $\quad 8846.22826 \quad 705.67325$ 


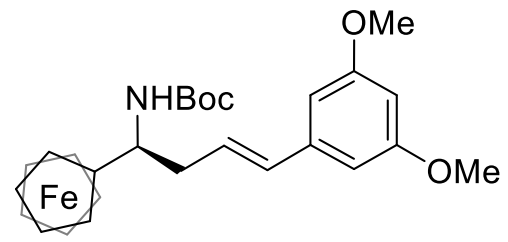

$\left(15 \mathbf{e}^{\prime}\right)$

Data File E: \DATA $\backslash$ LYN $\backslash$ LN-4-17 2019-12-04 20-36-21 LYN-4-171.D

Sample Name: LYN-4-17-RAC

\begin{tabular}{|c|c|c|c|}
\hline Acq. Operator & : SYSTEM & Seq. Line : & 2 \\
\hline Acq. Instrument & : 1260 & Location : & 4 \\
\hline Injection Date & : 12/4/2019 8:48:49 PM & Inj : & 1 \\
\hline
\end{tabular}

Ácq. Method : E: \DATA \LYN LYN-4-17 2019-12-04 20-36-21 \ADH-90-10-40min-254nm-1ML-10ul-RAC

Last changed : $12 / 4 / 20198: 36: 21$ PM by SYSTEM

Analysis Method : E: \DATA LYN LYN-4-17 2019-12-04 20-36-21\ADH-90-10-40min-254nm-1ML-10ul-RAC .M (Sequence Method)

Last changed : 7/24/2020 9:41:05 AM by SYSTEM

(modified after loading)

Additional Info : Peak (s) manually integrated

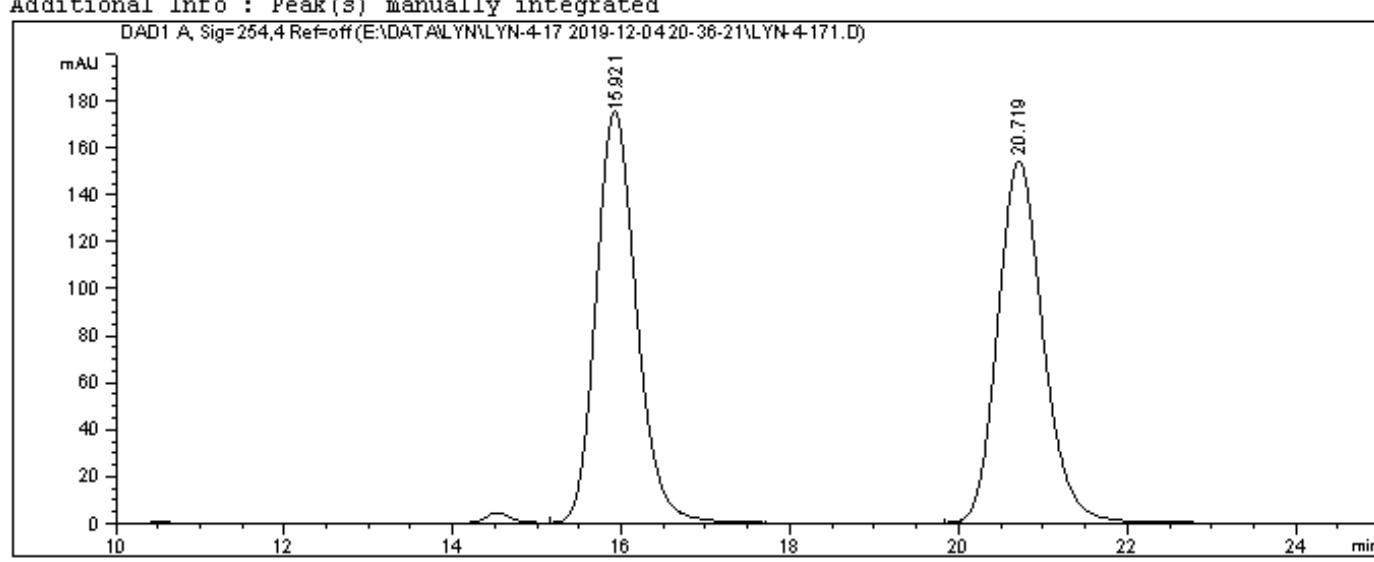

Area Percent Report

$\begin{array}{lll}\text { Sorted BY } & : & \text { Signal } \\ \text { Multiplier } & : & 1.0000 \\ \text { Dilution } & : & 1.0000\end{array}$

Do not use Multiplier \& Dilution Factor with ISTDs

Signal 1: DADl A, Sig=254, 4 Ref=off

\begin{tabular}{|c|c|c|c|c|c|}
\hline $\begin{array}{c}\text { Peak } \\
\quad \#\end{array}$ & $\begin{array}{l}\text { RetTime Type } \\
\text { [min] }\end{array}$ & $\begin{array}{l}\text { Width } \\
\text { [min] }\end{array}$ & $\begin{array}{c}\text { Area } \\
\text { [mAUt's] }\end{array}$ & $\begin{array}{l}\text { Height } \\
\text { [mAU] }\end{array}$ & $\begin{array}{c}\text { Area } \\
\stackrel{8}{*}\end{array}$ \\
\hline 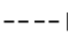 & -----1 & ----- & --------- & 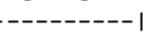 & --2 \\
\hline 1 & $15.921 \mathrm{BB}$ & 0.5156 & 5838.72705 & 175.27948 & 50.4038 \\
\hline 2 & $20.719 \mathrm{BB}$ & 0.5593 & 5745.17725 & 154.34244 & 49.5962 \\
\hline Tota & : & & $1.15839 \mathrm{e} 4$ & 329.62192 & \\
\hline
\end{tabular}

ホホホ End of Report 
Data File E: \DATA LYN LYN-4-18 2019-12-04 21-45-49 LYN-4-18.D

Sample Name: LYN-4-18-GH

Acq. Operator : SYSTEM

Ac q. Instrument : 1260

Injection Date : 12/4/2019 9:47:18 PM

Acq. Method . $\mathrm{M}$

Last changed : 12/4/2019 9:45:49 PM by SYSTEM

Analysis Method : E: \DATA LYN LYN-4-18 2019-12-04 21-45-49 \ADH-90-10-40min-254nm-1ML-10ul-RAC .M (Sequence Method)

Last changed : 7/24/2020 10:16:33 AM by SYSTEM

(modified after loading)

Additional Info : Peak (s) manually integrated

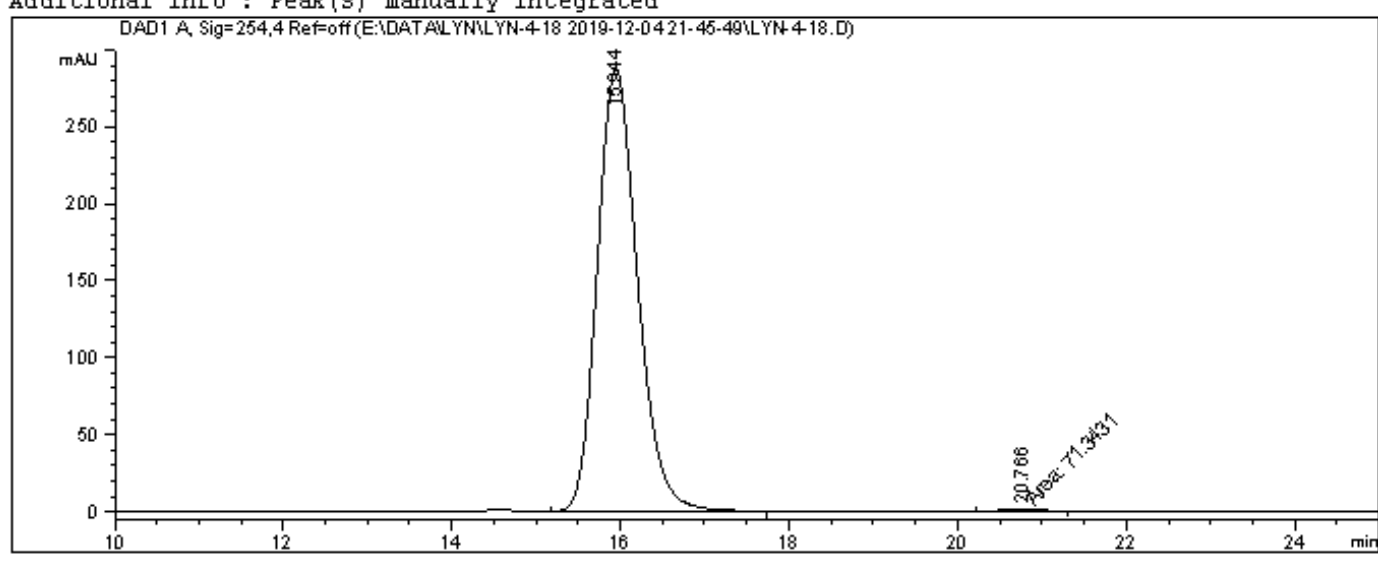

\section{Area Percent Report}

\begin{tabular}{|c|c|}
\hline Sorted By & Signal \\
\hline Multiplier & 1.0000 \\
\hline Dilution & $: \quad 1.0000$ \\
\hline
\end{tabular}

Do not use Multiplier \& Dilution Factor with ISTDs

Signal 1: DADl A, Sig=254,4 Ref=off

\begin{tabular}{|c|c|c|c|c|c|c|}
\hline $\begin{array}{c}\text { Peak } \\
\#\end{array}$ & $\begin{array}{c}\text { RetTime } \\
\text { [min] }\end{array}$ & Trpe & $\begin{array}{l}\text { Width } \\
\text { [min] }\end{array}$ & $\begin{array}{c}\text { Area } \\
{\left[\mathrm{mAU} \mathrm{U}^{*} \mathrm{~s}\right]}\end{array}$ & $\begin{array}{l}\text { Height } \\
\text { [mAU] }\end{array}$ & $\begin{array}{c}\text { Area } \\
\stackrel{5}{*}\end{array}$ \\
\hline- & & & & --- & & \\
\hline 1 & & & & 9596 & 288 & 621 \\
\hline 2 & 20.7 & M & 559 & 71.34306 & 1.81285 & 0.7379 \\
\hline
\end{tabular}

Totals :

9668.07939290 .14158 
<smiles>N[C@@H](CCc1ccccc1C(=O)O)c1ccccc1</smiles>

$\left(15 f^{\prime}\right)$

Data File E: \DATA $\backslash$ LYN $\backslash$ LN-4-8285 2019-12-19 16-51-14\LYN-4-82853.D Sample Name: LYN-4-85-RAC

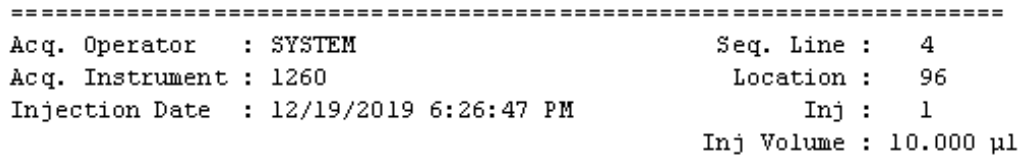

Ac q. Method : E: \DATA $\backslash$ LYN LYN-4-8285 2019-12-19 16-51-14\ADH-90-10-40min-254nm-1ML-10ulRAC.M

Last changed : 12/19/2019 4:51:14 PM by SYSTEM

Analysis Method : E: \DATA LYN LYN-4-8285 2019-12-19 16-51-14\ADH-90-10-40min-254nm-1ML-10ulRAC.M (Sequence Method)

Last changed : 7/24/2020 9:15:28 AM by SYSTEM (modified after loading)

Additional Info: Peak (s) manually integrated

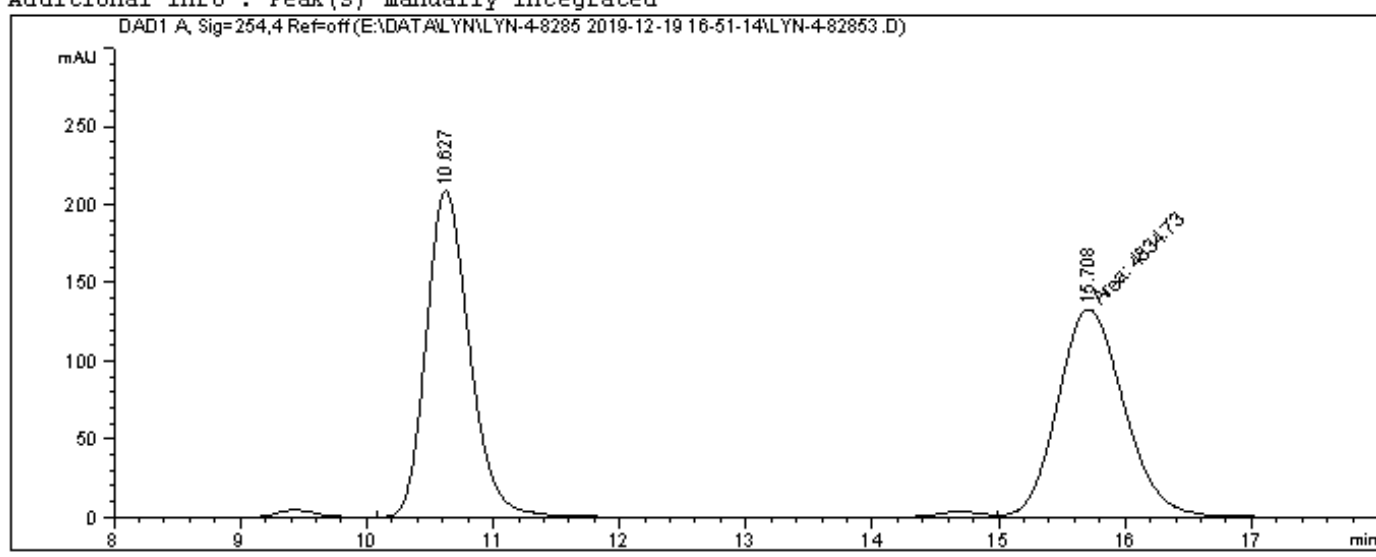

Area Percent Report

\begin{tabular}{|c|c|c|}
\hline Sorted By & : & Signal \\
\hline Multiplier & : & 1.0000 \\
\hline Dilution & : & 1.0000 \\
\hline
\end{tabular}

Do not use Multiplier \& Dilution Factor with ISTDs

Signal 1: DADl A, Sig=254, 4 Ref=off

\begin{tabular}{|c|c|c|c|c|c|c|}
\hline $\begin{array}{c}\text { Peak } \\
\#\end{array}$ & $\begin{array}{c}\text { RetTime } \\
\text { [min] }\end{array}$ & Type & $\begin{array}{l}\text { Width } \\
\text { [min] }\end{array}$ & 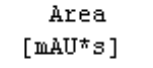 & $\begin{array}{l}\text { Height } \\
\text { [mAU] }\end{array}$ & $\begin{array}{c}\text { Area } \\
\stackrel{5}{8}\end{array}$ \\
\hline & & & & --- & & \\
\hline 1 & 10.627 & $\mathrm{BB}$ & 0.3592 & 4960.92188 & 208.79193 & 50.6441 \\
\hline 2 & 15.708 & FM & 0.6055 & 4834.73389 & 133.07823 & 49.3559 \\
\hline ot & : & & & 9795.65576 & 341.87016 & \\
\hline
\end{tabular}

ネネ End of Report ネネ 
Data File E: \DATA \LM LYN-4-8285 2019-12-19 16-51-14\L YN-4-82854.D

Sample Name: LYN-4-85-GH

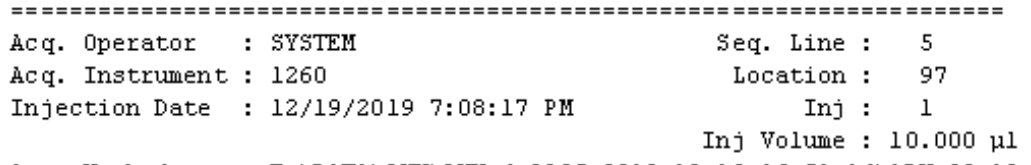

Acq. Method : E: \DATA $\backslash$ LYN $\backslash$ LYN-4-8285 2019-12-19 16-51-14 RAC.M

Last changed : 12/19/2019 4:51:14 PM by SYSTEM

Analysis Method : E: \DATA LYN LYN-4-8285 2019-12-19 16-51-14\ADH-90-10-40min-254nm-1ML-10ulRAC.M (Sequence Method)

Last changed : 7/24/2020 9:17:10 AM by SYSTEM

(modified after loading)

Additional Info : Peak (s) manually integrated

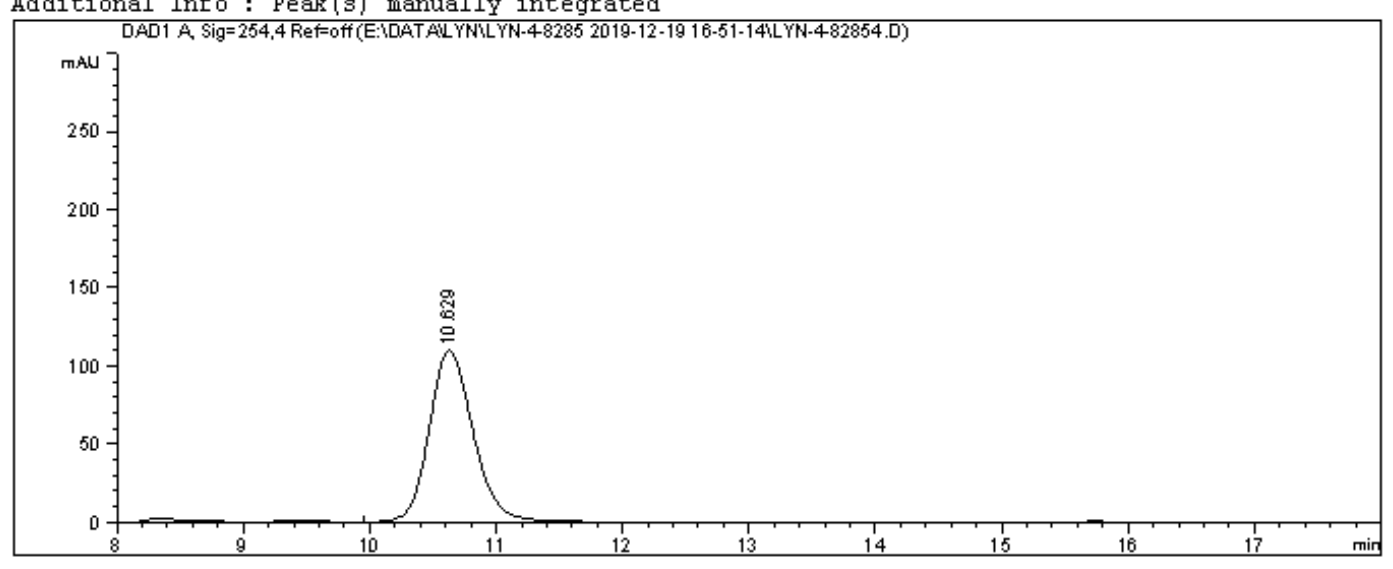

Area Percent Report

\begin{tabular}{|c|c|c|}
\hline Sorted By & : & Signal \\
\hline Multiplier & : & 1.0000 \\
\hline Dilution & : & 1.0000 \\
\hline
\end{tabular}

Do not use Multiplier \& Dilution Factor with ISTDs

Signal 1: DẢDl Å, Sig=254,4 Ref=off

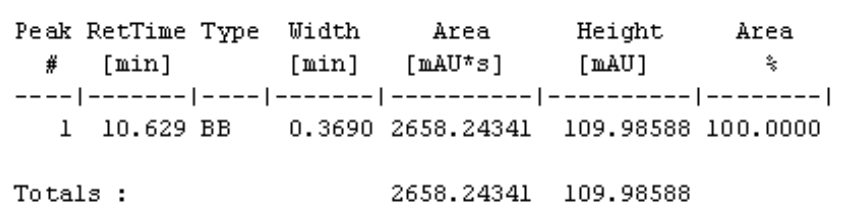

ネネー End of Report *ネ* 


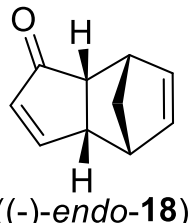

Data $F$ ile D: $\backslash G C \backslash C X D A T A C X-13-31-5 \backslash C X-13-31-5 \quad 2019-12-17 \quad 10-35-09 \backslash 101 F 0101 . D$ Sample Ilame: CX-13-31-S-RAC

\begin{tabular}{|c|c|c|}
\hline Acq. Operator & $: \quad x 0$ & Seq. L іле : \\
\hline q. Inst rument & : Inst rument 2 & Locatior: Vial 101 \\
\hline Injection Date & $: 17-$ Dec-19, $10: 36: 46$ & $\begin{array}{rlc}\text { Iлj : } & 1 \\
\text { Iлj Volume } & 1 & 1\end{array}$ \\
\hline
\end{tabular}

ACq. Method : D: $\backslash$ GC CX $\backslash$ DATA $\backslash C X-13-31-S \backslash C X-13-31-S \quad 2019-12-17$ 10-35-09\CS-1000-180C-1ML1- 10 MII.M. M

Last changed : $12 / 17 / 201910: 35: 32$ M bY X0

(modif ied after loading)

AralYs is Method : D: \GC XOQMETHOD CHIRAL-SELECT 1000-200C-1ML .M

Last changed : 1/14/2020 11:38:25 MM by XO

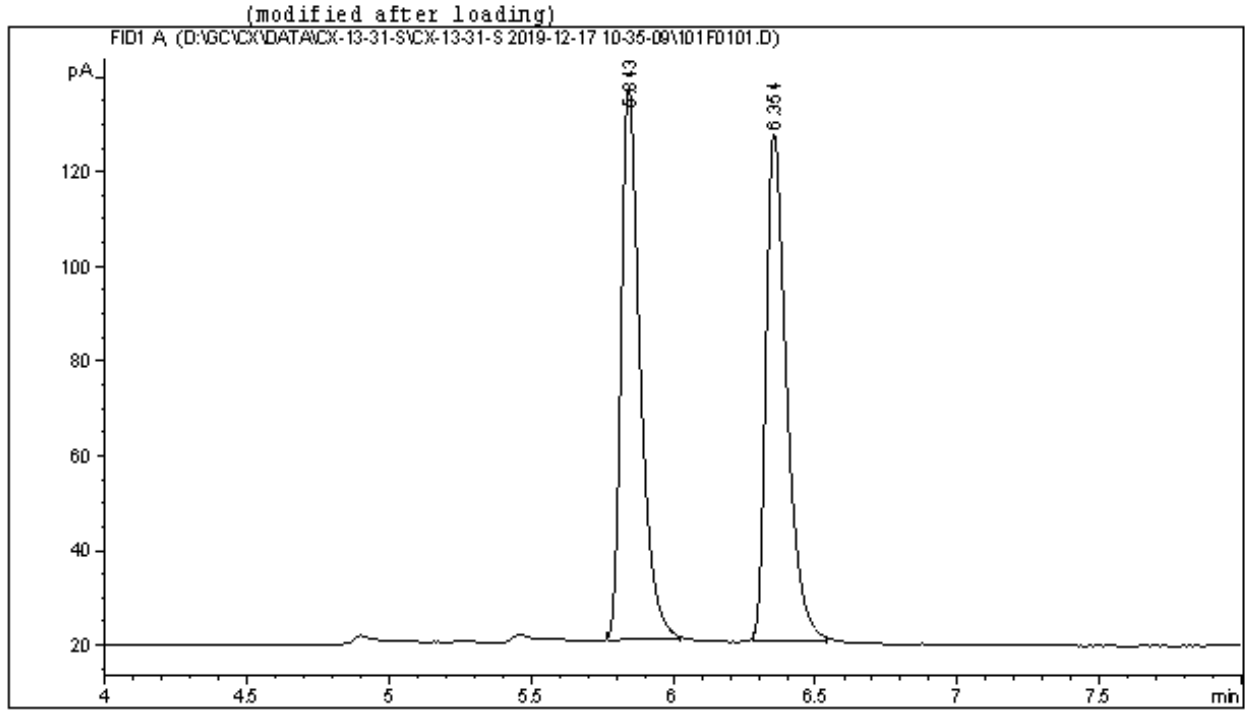

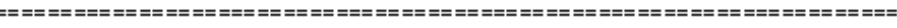

Area Percent Report

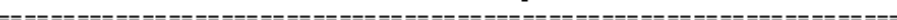

$\begin{array}{lll}\text { Sorted BY } & : & \text { Signal } \\ \text { Multiplier } & : & 1.0000 \\ \text { Dilution } & : & 1.0000\end{array}$

Dilution : 1.0000

Use Multiplier \& Dilution Factor with ISTDs

Signal l: FID $A$,

\begin{tabular}{|c|c|c|c|c|c|}
\hline $\begin{array}{c}\text { Peak } \\
\#\end{array}$ & $\begin{array}{l}\text { RetT ime TYpe } \\
\text { [miл] }\end{array}$ & $\begin{array}{l}\text { Width } \\
\text { [min] }\end{array}$ & $\begin{array}{r}\text { hrea } \\
{\left[\mathrm{pA}^{*} \mathrm{~s}\right]}\end{array}$ & $\begin{array}{l}\text { Height } \\
{[\mathrm{pA}]}\end{array}$ & $\begin{array}{c}\text { Area } \\
\text { 믑 }\end{array}$ \\
\hline---1 & -------|-- & $------\mid$ & --- & --- & |------- | \\
\hline 1 & $5.843 \mathrm{BB}$ & 0.0683 & 543.81274 & 116.74076 & 50.12880 \\
\hline 2 & $6.354 \mathrm{BB}$ & 0.0751 & 541.01825 & 106.80985 & 49.87120 \\
\hline 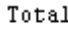 & & & 1084.83099 & 223.55061 & \\
\hline
\end{tabular}




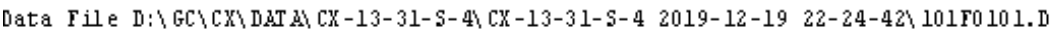
Sample Iame: CX-13-31-S-4

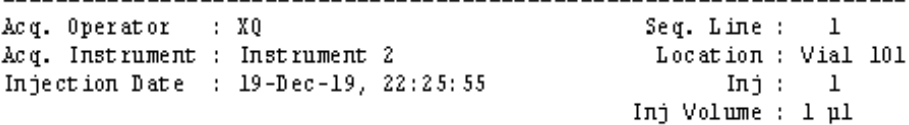

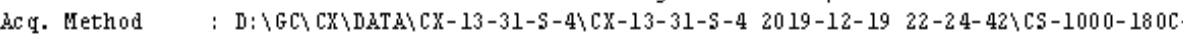
IML-1-10MIN .M

Last charged : $12 / 19 / 201910: 25: 01$ PM by X0

(modified after loading)

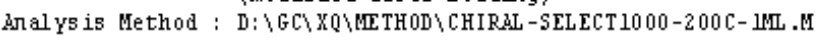

Last charged : 1/14/2020 11:41:57 HM bY X0

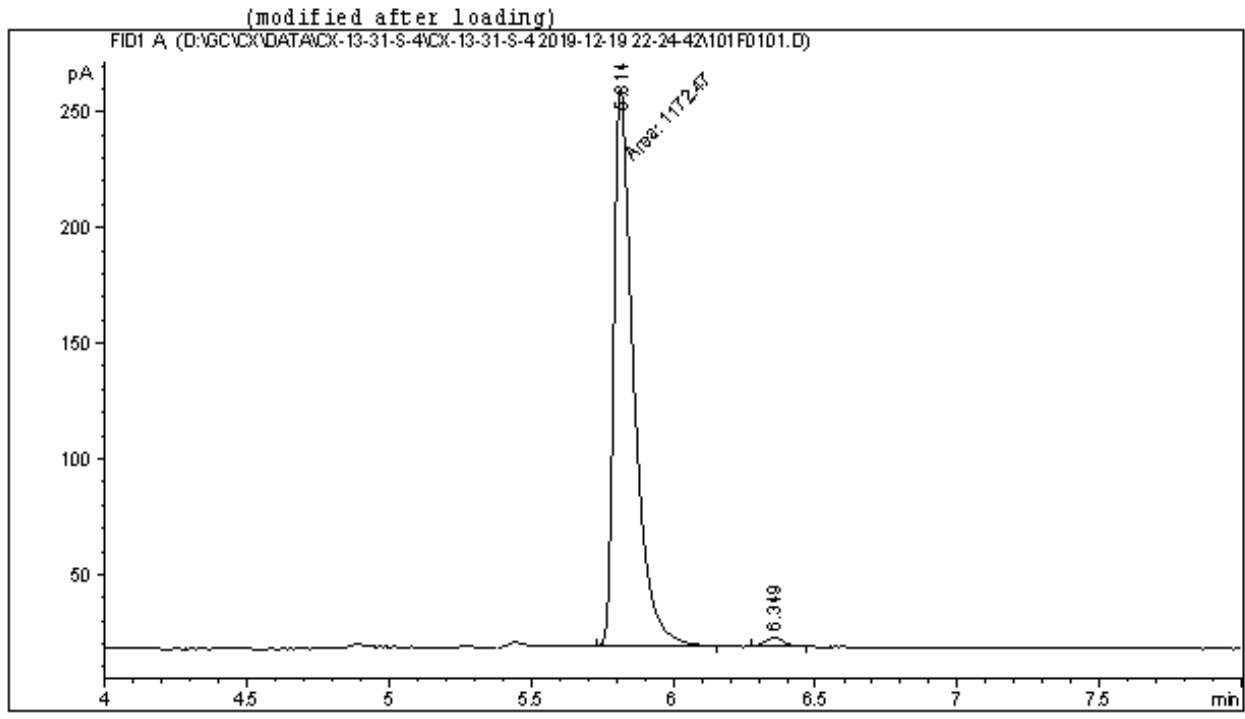

Area Percent Report

$\begin{array}{lll}\text { Sorted BY } & : & \text { Signal } \\ \text { Multiplier } & : & 1.0000 \\ \text { Dilution } & : & 1.0000\end{array}$

Use Multiplier s Dilution Factor with ISTDs

Signal l: FID 1 ,

\begin{tabular}{|c|c|c|c|c|c|}
\hline $\begin{array}{c}\text { Peak } \\
\#\end{array}$ & $\begin{array}{l}\text { RetT ime TYpe } \\
\text { [miл] }\end{array}$ & $\begin{array}{l}\text { Width } \\
\text { [mig] }\end{array}$ & $\begin{array}{r}\text { hrea } \\
{\left[\mathrm{pA}^{*} \mathrm{~s}\right]}\end{array}$ & $\begin{array}{l}\text { Height } \\
{[\mathrm{pd}]}\end{array}$ & $\begin{array}{c}\text { Area } \\
\text { 몹 }\end{array}$ \\
\hline----1 & $|------|--$ & ------ & |--------- & -1---n- & |--------| \\
\hline 1 & $5.814 \mathrm{MM}$ & 0.0812 & 1172.46655 & 240.71777 & 98.48115 \\
\hline 2 & $6.349 \mathrm{BB}$ & 0.0586 & 18.08265 & 3.86438 & 1. 51885 \\
\hline ot & : & & 1190.54920 & 244.58215 & \\
\hline
\end{tabular}




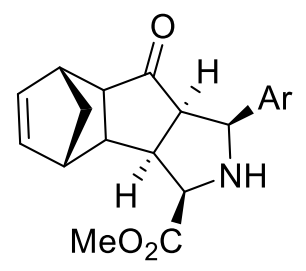

$\mathrm{Ar}=p-\mathrm{Cl}-\mathrm{C}_{6} \mathrm{H}_{4}$

(endo-endo-20)

Data File E: \DATA $\backslash$ CX...E-0PT 2020-01-07 17-36-17 CX-13-DUJJ-31-P-RAC-32-5-P-12-14-Me-opt1.D Sample Name: CX-13-DWJ-31-P-RAC

\begin{tabular}{|c|c|c|c|}
\hline Acq. Operator & : SYSTEM & seq. Line : & 2 \\
\hline Acq. Instrument & : 1260 & Location : & 31 \\
\hline Injection Date & $: 1 / 7 / 2020 \quad 5: 48: 50 \mathrm{PM}$ & $\begin{array}{r}\text { Inj : } \\
\text { Inj Volume : }\end{array}$ & $\begin{array}{l}1 \\
.000\end{array}$ \\
\hline
\end{tabular}

Acq. Method : E: \DATA $\backslash C X \backslash C X-13 \backslash C X-13-D$ JJJ-31-P-RAC-32-5-P-12-14-Me-opt 2020-01-07 17-36-17 (2-ADH-90-10-1. 5ML-206MM (ALL) -30MIN. M

Last changed : 1/7/2020 5:36:17 PM by SYSTEM

Analysis Method : E: \DATA CX CX-13 CX-13-DUJJ-31-P-RAC-32-5-P-12-14-Me-opt 2020-01-07 17-36-17 12-ADH-90-10-1.5ML-206MM (ALL)-30MIN.M (Sequence Method)

Last changed : 1/7/2020 10:31:08 PM by SYsTEM

(modified after loading)

Additional Info : Peak (s) manually integrated

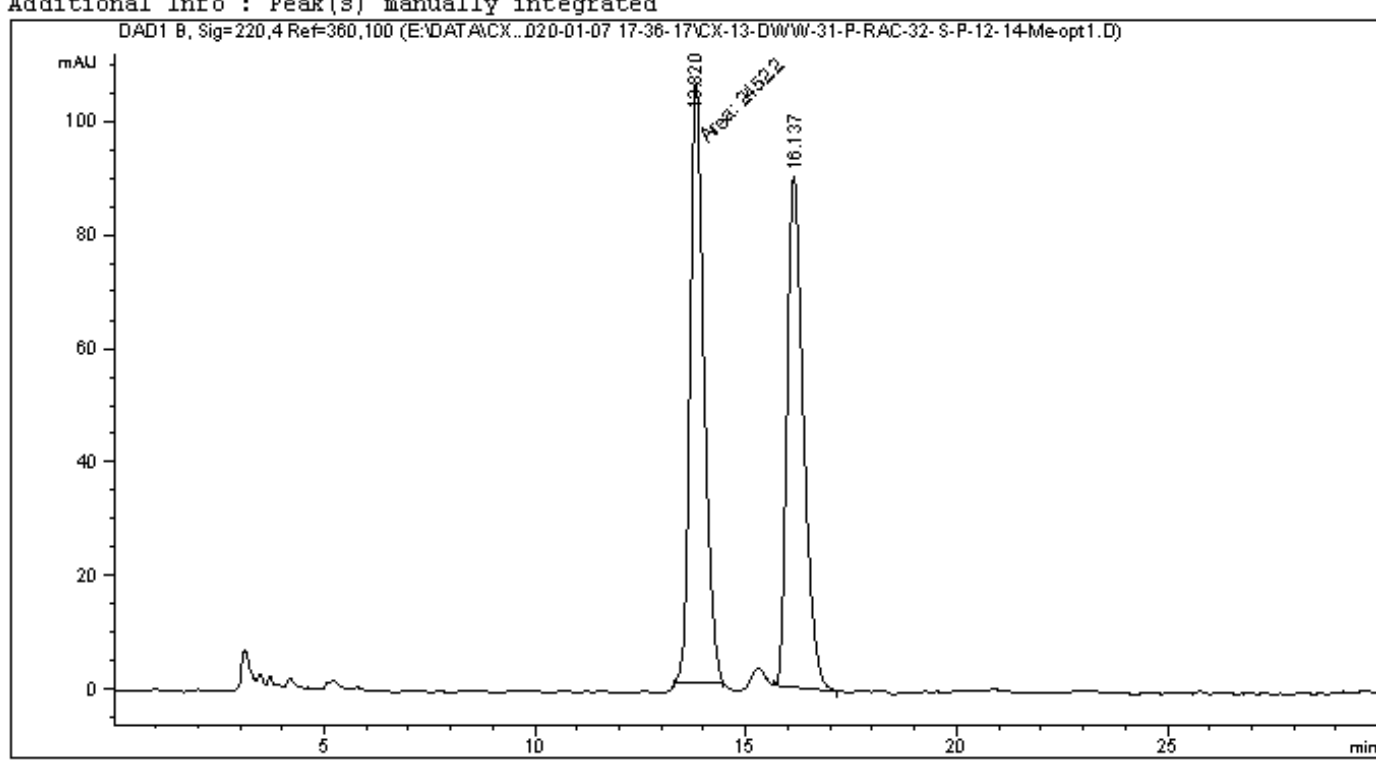

Area Percent Report

$\begin{array}{lll}\text { Sorted BY } & : & \text { Signal } \\ \text { Multiplier } & : & 1.0000 \\ \text { Dilution } & : & 1.0000\end{array}$

Do not use Multiplier \& Dilution Factor with IsTDs

Signal 1: DAD1 B, Sig $=220,4$ Ref $=360,100$

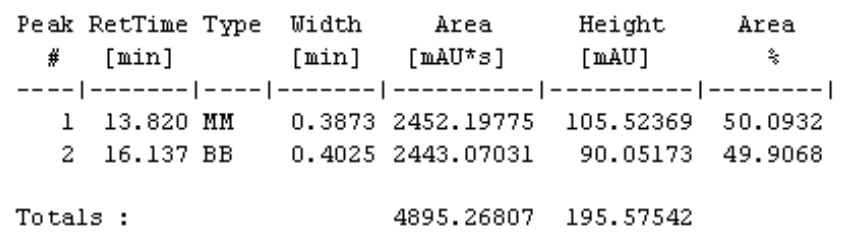


Data File E: \DATA $\backslash \mathrm{CX} \backslash \mathrm{CX}-13 \backslash \mathrm{CX}-13-31-\mathrm{P}-32-\mathrm{S}-\mathrm{P}$ 2019-12-16 22-50-24\CX-13-31-P-32-S-P1.D Sample Name: CX-13-31-P
Acq. Operator : SYSTEM
Acq. Instrument : 1260
seq. Line : 2
Injection Date : 12/16/2019 11:02:54 PM
Location : 34
Inj : 1
Inj Volume : $5.000 \mu \mathrm{l}$

Ac q. Method : E: \DATA $\backslash$ CX $C X-13 \backslash C X-13-31-P-32-5-P$ 2019-12-16 22-50-24\2-ADH-90-10-1.5ML$206 \mathrm{MM}$ (ALL) -30MIN. M

Last changed : 12/16/2019 10:50:24 PM by sYsTEM

Analysis Method : E: \DATA CX CX-13\CX-13-31-P-32-S-P 2019-12-16 22-50-24\2-ADH-90-10-1.5ML206MM(ALL)-30MIN.M (Sequence Method)

Last changed : 1/7/2020 10:34:25 PM by SYSTEM

(modified after loading)

Additional Info : Peak (s) manually integrated

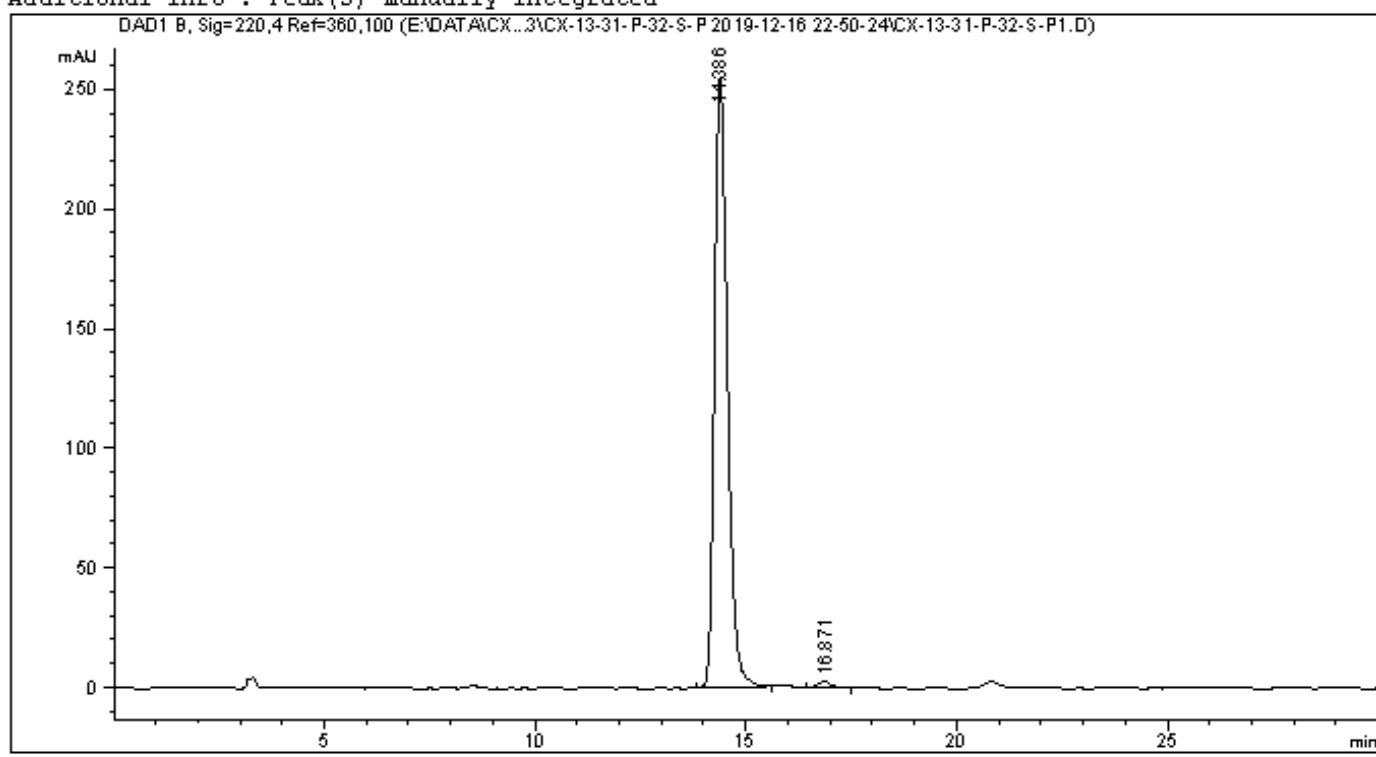

Area Percent Report

\begin{tabular}{|c|c|}
\hline Sorted BY & Signal \\
\hline Multiplier & 1.0000 \\
\hline Dilution & 1.0000 \\
\hline
\end{tabular}

Signal 1: DADl B, Sig=220, 4 Ref $=360,100$

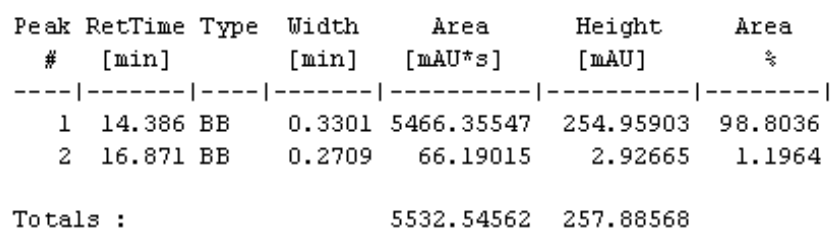


<smiles>O=C1C(c2ccccc2)=CC2CC3C=CC2C13</smiles>

$((-)-$ exo-21)

Data File E: \DATA $\backslash$ CX $\backslash$ CX-13 $\backslash$ CX-13-32-5-RAC 2020-01-09 00-38-20 CX-13-32-5-RAC3.D Sample Name: CX-13-32-S-RAC
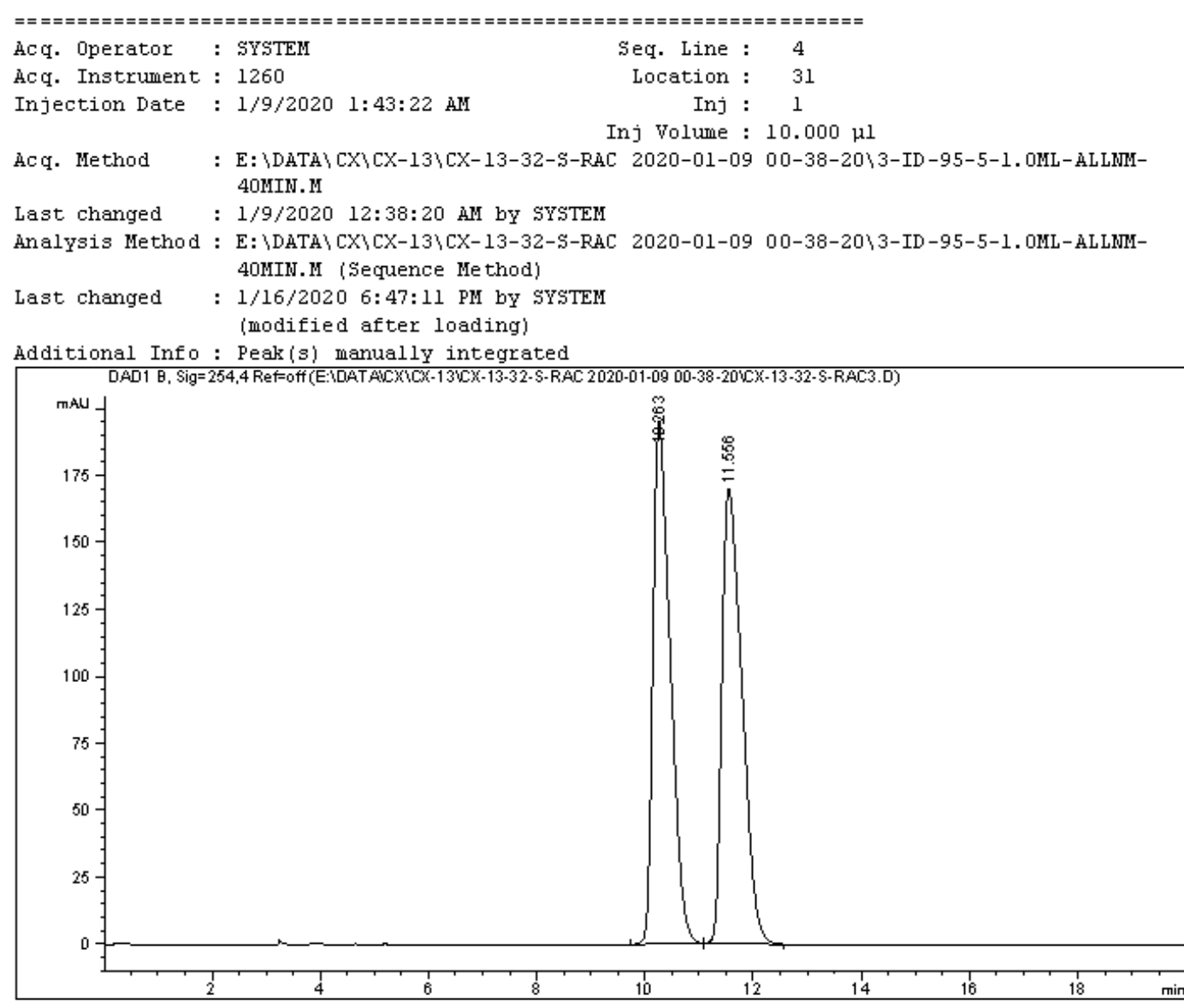

Area Percent Report

\begin{tabular}{|c|c|c|c|c|}
\hline Sorted BY & : & \multicolumn{3}{|l|}{ Signal } \\
\hline Multiplier & : & \multicolumn{3}{|l|}{1.0000} \\
\hline Dilution & : & \multicolumn{3}{|l|}{1.0000} \\
\hline \multicolumn{5}{|c|}{ Do not use Multiplier \& Dilution Factor with ISTDs } \\
\hline \multicolumn{5}{|c|}{ Signal 1: DADl B, Sig=254,4 Ref=off } \\
\hline $\begin{array}{l}\text { Peak RetTime Type } \\
\# \quad \text { [min] }\end{array}$ & $\begin{array}{l}\text { Width } \\
\text { [min] }\end{array}$ & $\begin{array}{c}\text { Area } \\
{\left[\mathrm{mAH}^{*} \mathrm{~s}\right]}\end{array}$ & $\begin{array}{l}\text { Height } \\
\text { [mAU] }\end{array}$ & $\begin{array}{c}\text { Area } \\
\stackrel{\phi}{8}\end{array}$ \\
\hline$----|-------|---$ & ------1 & ---------1 & ---------1 & -------1 \\
\hline $110.263 \mathrm{BB}$ & 0.3296 & 4358.96533 & 195.15413 & 50.0011 \\
\hline $211.556 \mathrm{BB}$ & 0.3833 & 4358.77832 & 169.89032 & 49.9989 \\
\hline Totals : & & 8717.74365 & 365.04445 & \\
\hline
\end{tabular}


Data File E: \DATA $\backslash$ CX CX-13 CX-13-32-S-RAC-0PT 2020-01-09 08-00-19 CX-13-32-S-RAC-optl.D Sample Name: CX-13-32-S-0PT

\begin{tabular}{|c|c|c|c|}
\hline Acq. Operator & : SYSTEM & Seq. Line : & 2 \\
\hline Acq. Instrument & : 1260 & Location : & 32 \\
\hline Injection Date & : $1 / 9 / 2020 \quad 8: 12: 44 \mathrm{AM}$ & $\begin{array}{r}\text { Inj : } \\
\text { Inj Volume : }\end{array}$ & $\begin{array}{l}1 \\
0.000 \mu 1\end{array}$ \\
\hline
\end{tabular}

Acq. Method : E: \DATA $\backslash \mathrm{CX} \backslash \mathrm{CX}-13 \backslash \mathrm{CX}-13-32-5-R A C-0 P T$ 2020-01-09 08-00-19 3-ID-95-5-1. 0MLALLMM-40MIN.M

Last changed : 1/9/2020 8:00:19 AM by SYSTEM

Analysis Method : E: \DATA $\backslash$ CX CX-13 CX-13-32-S-RAC-0PT 2020-01-09 08-00-19\3-ID-95-5-1. 0MLALLMM-40MIN.M (Sequence Method)

Last changed : 1/16/2020 7:25:49 PM by SYSTEM

(modified after loading)

Additional Info : Peak (s) manually integrated

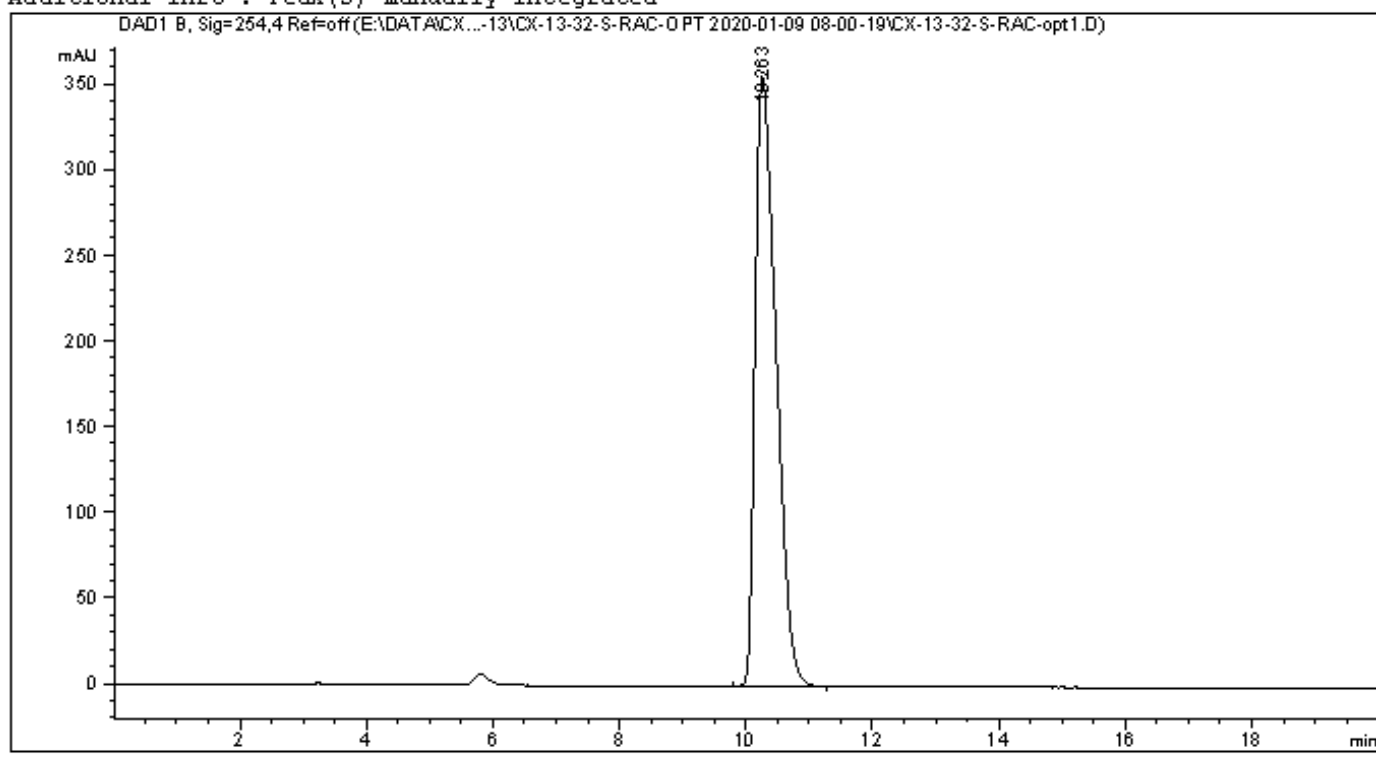

Area Percent Report

\begin{tabular}{|c|c|}
\hline Sorted BY & Signal \\
\hline Multiplier & 1.0000 \\
\hline Dilution & 1.0000 \\
\hline
\end{tabular}

Signal 1: DẢD B, Sig=254, 4 Ref=off

\begin{tabular}{|c|c|c|c|c|c|}
\hline $\begin{array}{c}\text { Peak } \\
\#\end{array}$ & $\begin{array}{l}\text { RetTime Type } \\
\text { [min] }\end{array}$ & $\begin{array}{l}\text { Width } \\
\text { [min] }\end{array}$ & $\begin{array}{c}\text { Area } \\
{\left[\mathrm{mAU}^{*} \mathrm{~s}\right]}\end{array}$ & $\begin{array}{l}\text { Height } \\
\text { [miU] }\end{array}$ & $\begin{array}{c}\text { Area } \\
\stackrel{4}{8}\end{array}$ \\
\hline & & & 8217 & & 100.0000 \\
\hline
\end{tabular}

Totals : $\quad 8217.56934 \quad 355.94278$ 


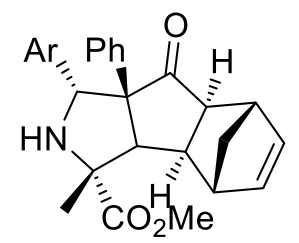

$$
\mathrm{Ar}=p-\mathrm{Cl}-\mathrm{C}_{6} \mathrm{H}_{4}
$$

(endo-exo-22)

Data File E: \DATA $\backslash$ CX $\backslash$ CX-9 CX-9-113-P-RAC 2018-08-28 13-48-45 CX-9-113-P-RAC.D Sample Name: CX-9-113-P-RAC

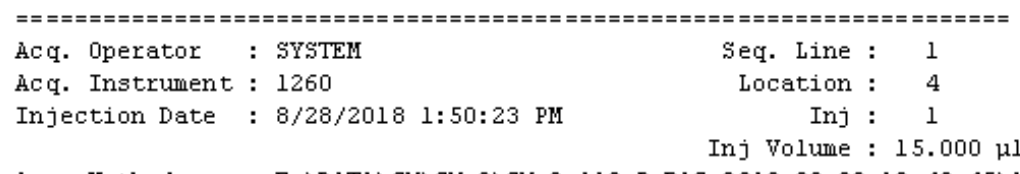

Acq. Method : E: \DATA $\backslash C X \backslash C X-9 \backslash C X-9-113-P-R A C$ 2018-08-28 13-48-45 4OMIN-ALL L.M

Last changed : 8/28/2018 1:48:45 PM by SYsTEM

Analysis Method : E: \DATA CX CX-9 CX-9-113-P-RAC 2018-08-28 13-48-45 ADH-90-10-1.5ML-206NM40MIN-ALL.M (Sequence Method)

Last changed : 8/28/2018 3:14:41 PM by SYSTEM

(modified after loading)

Additional Info : Peak (s) manually integrated

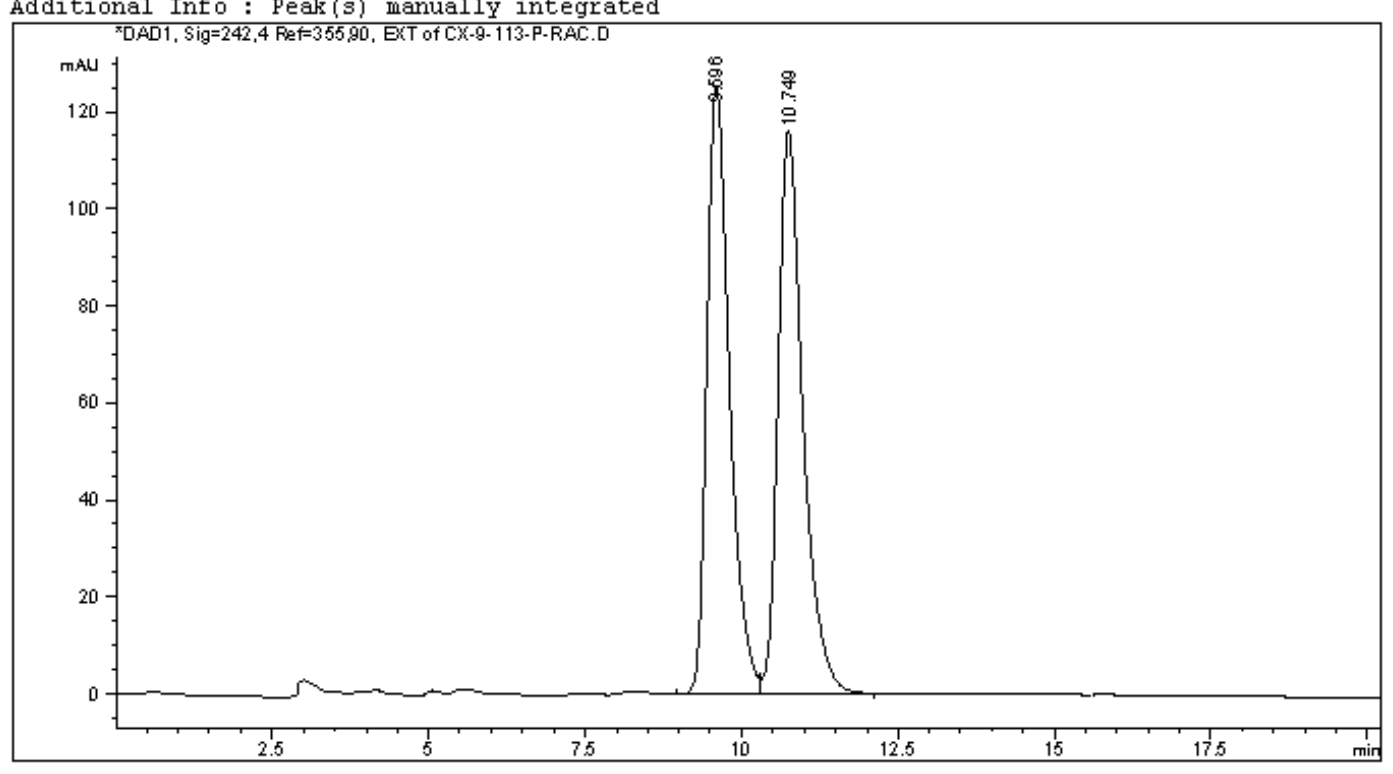

Area Percent Report

$\begin{array}{lll}\text { Sorted By } & : & \text { Signal } \\ \text { Multiplier } & : & 1.0000 \\ \text { Dilution } & : & 1.0000\end{array}$

Do not use Multiplier \& Dilution Factor with ISTDs

signal 1: DADl, sig=242,4 Ref $=355,90$, EXT

signal has been modified after loading from rawdata file!

\begin{tabular}{|c|c|c|c|c|c|c|}
\hline $\begin{array}{c}\text { Peak } \\
\#\end{array}$ & $\begin{array}{c}\text { RetTime } \\
\text { [min] }\end{array}$ & Type & $\begin{array}{l}\text { Width } \\
\text { [min] }\end{array}$ & $\begin{array}{c}\text { Area } \\
\text { [mAUt* } s]\end{array}$ & $\begin{array}{l}\text { Height } \\
\text { [miU] }\end{array}$ & $\begin{array}{c}\text { Area } \\
\stackrel{5}{*}\end{array}$ \\
\hline & & & . & ---- & $---------\mid$ & -------1 \\
\hline 1 & & $\mathrm{BV}$ & 0.3844 & 3160.24829 & 125.25153 & 49.6075 \\
\hline 2 & 10.749 & VB & 0.4160 & 3210.25684 & 115.97066 & 50.3925 \\
\hline
\end{tabular}

Totals : $\quad 6370.50513 \quad 241.22218$ 
Data File E: \DATA $\backslash$ CX...E-0PT 2020-01-07 17-36-17 CX-13-DUJJ-31-P-RAC-32-S-P-12-14-Me-opt3.D Sample Name: CX-13-DWJ-32-P-0PT

$\begin{array}{llrl}\text { Acq. Operator } & : \text { SYSTEM } & \text { Seq. Line : } & 4 \\ \text { Acq. Instrument }: 1260 & \text { Location }: & 35 \\ \text { Injection Date }: 1 / 7 / 2020 & 6: 51: 51 \mathrm{PM} & \text { Inj }: & 1 \\ & & \text { Inj Volume : } 5.000 \mu \mathrm{l}\end{array}$

Acq. Method : E: \DATA $\backslash C X \backslash C X-13 \backslash C X-13-D$ J6J-31-P-RAC-32-S-P-12-14-Me-opt 2020-01-07 17-36-17 (2-ADH-90-10-1. 5ML-206MN (ALL) -30MIN.M

Last changed : 1/7/2020 5:36:17 PM by SYSTEM

Analysis Method : E: \DATA $\backslash$ CX CX-13 CX-13-D价-31-P-RAC-32-5-P-12-14-Me-opt 2020-01-07 17-36-17 12-ADH-90-10-1.5ML-206MM(ALL)-30MIN.M (Sequence Method)

Last changed : 1/7/2020 10:49:07 PM by SYSTEM

(modified after loading)

Additional Info: Peak (s) manually integrated

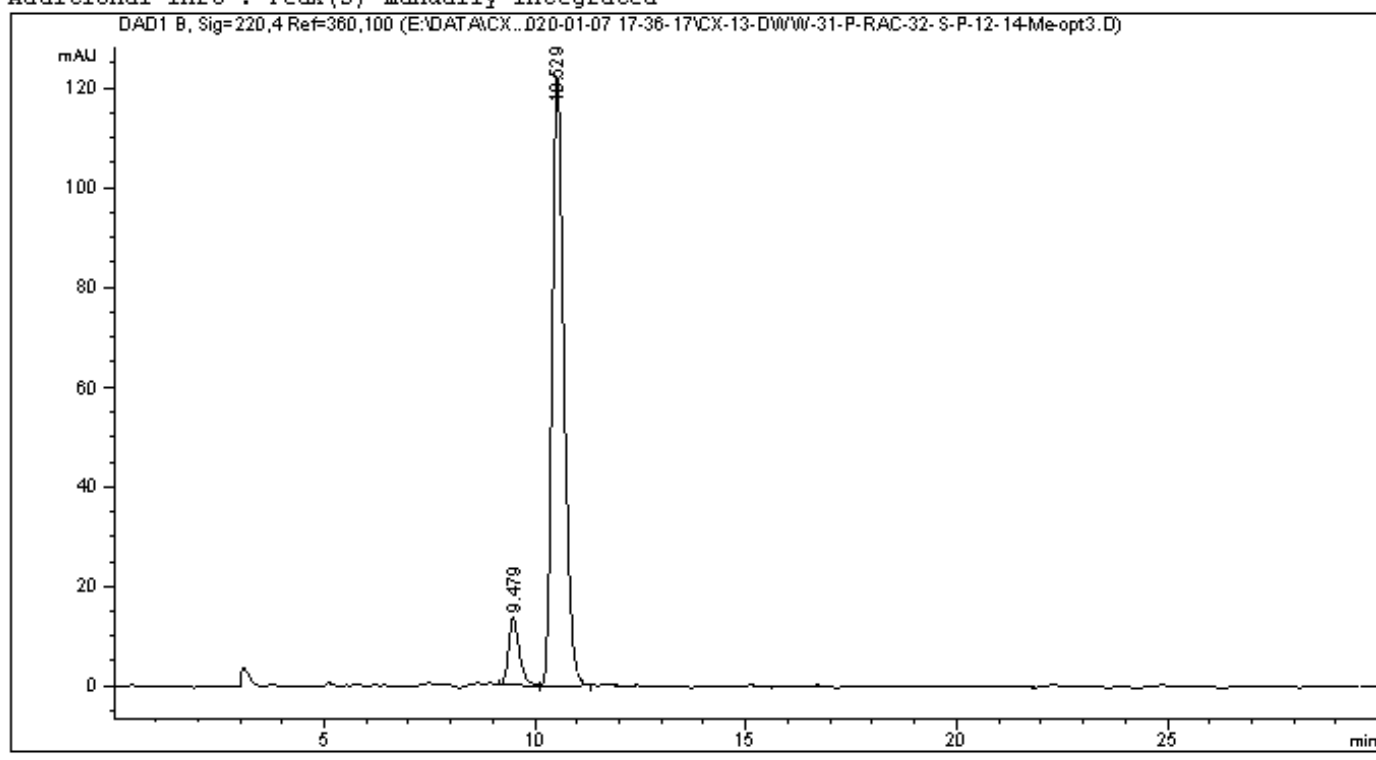

Area Percent Report

\begin{tabular}{|c|c|c|}
\hline Sorted BY & : & signe \\
\hline Multiplier & : & 000 \\
\hline Dilution & : & 1.00 \\
\hline
\end{tabular}

Do not use Multiplier \& Dilution Factor with ISTDs

Signal 1: DADl B, Sig $=220,4$ Ref $=360,100$

\begin{tabular}{|c|c|c|c|c|c|c|}
\hline $\begin{array}{c}\text { Peak } \\
\#\end{array}$ & $\begin{array}{c}\text { RetTime } \\
\text { [min] }\end{array}$ & Type & $\begin{array}{l}\text { Width } \\
\text { [min] }\end{array}$ & $\begin{array}{c}\text { Àrea } \\
\text { [mAU*'s] }\end{array}$ & $\begin{array}{l}\text { Height } \\
\text { [mAU] }\end{array}$ & $\begin{array}{c}\text { Àrea } \\
\text { के }\end{array}$ \\
\hline & & & 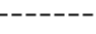 & ------ & -------- & - \\
\hline 1 & 9.479 & $\mathrm{BB}$ & 0.2606 & 240.16885 & 13.49734 & 9.0861 \\
\hline 2 & 10.529 & $\mathrm{BB}$ & 0.2964 & 2403.09912 & 122.23522 & 90.9139 \\
\hline
\end{tabular}

Totals : $\quad 2643.26797 \quad 135.73256$ 
<smiles>CC[C@H](CCc1ccccc1)CC(=O)OC</smiles>

Data File E: \DATA \D相 Sample Name: dor-9-78-rac

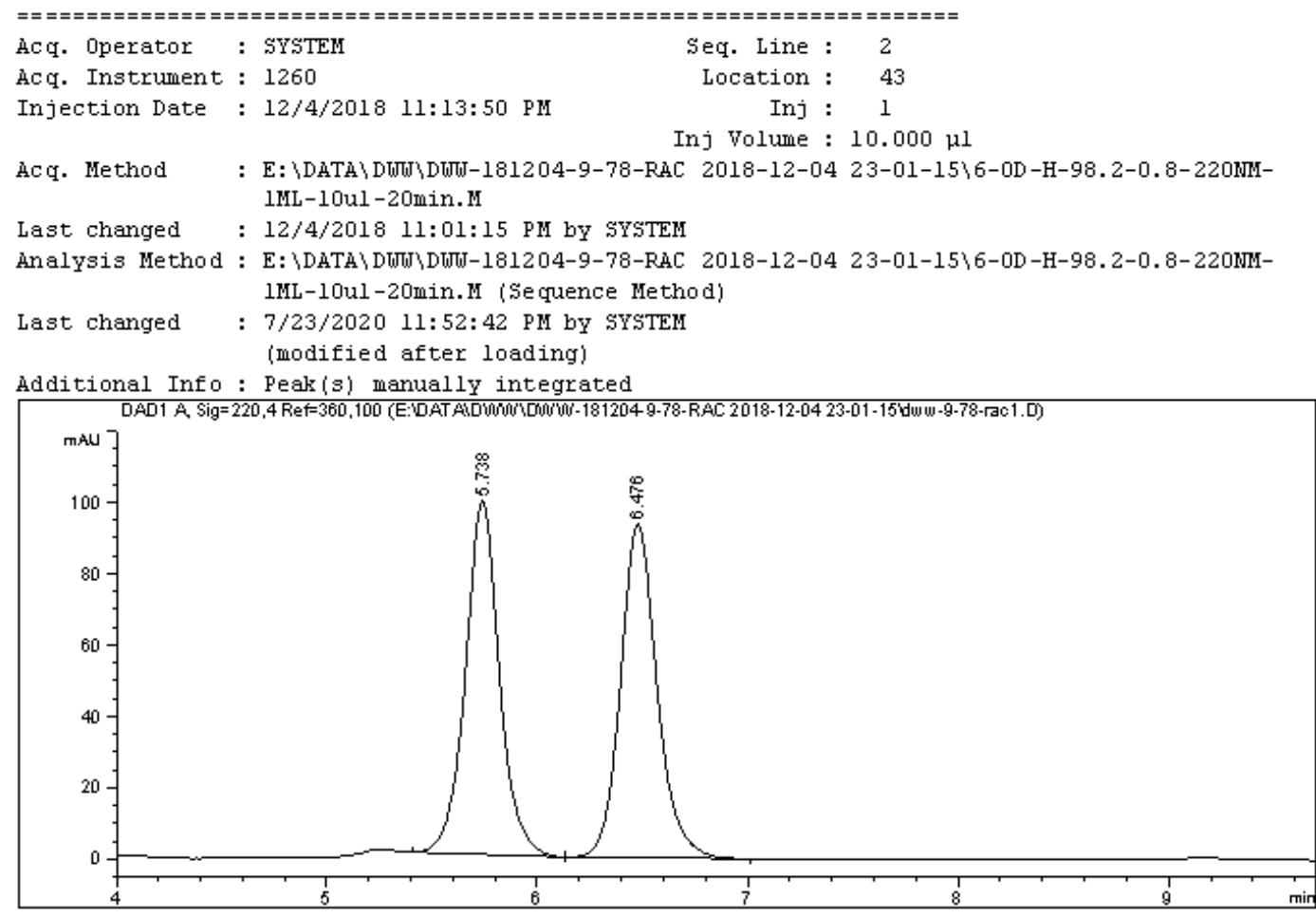

Area Percent Report

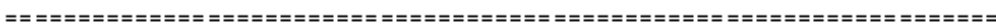

Sorted By : Signal

Multiplier : 1.0000

Dilution : 1.0000

Do not use Multiplier \& Dilution Factor with ISTDs

Signal 1: DADl A, Sig $=220,4$ Ref $=360,100$

\begin{tabular}{|c|c|c|c|c|c|}
\hline $\begin{array}{c}\text { Peak } \\
\#\end{array}$ & $\begin{array}{l}\text { RetTime Type } \\
\text { [min] }\end{array}$ & $\begin{array}{l}\text { Width } \\
\text { [min] }\end{array}$ & $\begin{array}{c}\text { Area } \\
{\left[\mathrm{m} \mathrm{AU}^{*} \mathrm{~S}\right]}\end{array}$ & $\begin{array}{l}\text { Height } \\
\text { [mAU] }\end{array}$ & $\begin{array}{c}\text { Area } \\
\%\end{array}$ \\
\hline & & & & & \\
\hline 1 & $38 \mathrm{BB}$ & 0.1681 & 1113.85303 & 99.13763 & 6502 \\
\hline 2 & $6.476 \mathrm{BB}$ & 0.1837 & 1129.54639 & 93.63854 & 50.3498 \\
\hline
\end{tabular}

Totals : $\quad 2243.39941 \quad 192.77618$ 


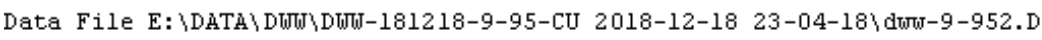
Sample Name: CuBF4
Acq. Operator : SYSTEM
Seq. Line : 3
Acq. Operator : SYSTE
Injection Date : 12/18/2018 11:38:15 PM
Location : 94
Inj : 1
Inj Volume : $10.000 \mu l$

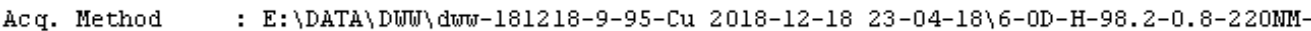
IML-10ul-20min. M

Last changed : 12/18/2018 11:04:18 PM by SYSTEM

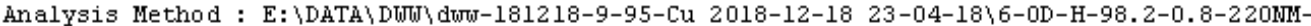
1ML-10ul-20min.M (Sequence Method)

Last changed : 7/23/2020 11:56:17 PM by SYSTEM

(modified after loading)

Additional Info : Peak (s) manually integrated

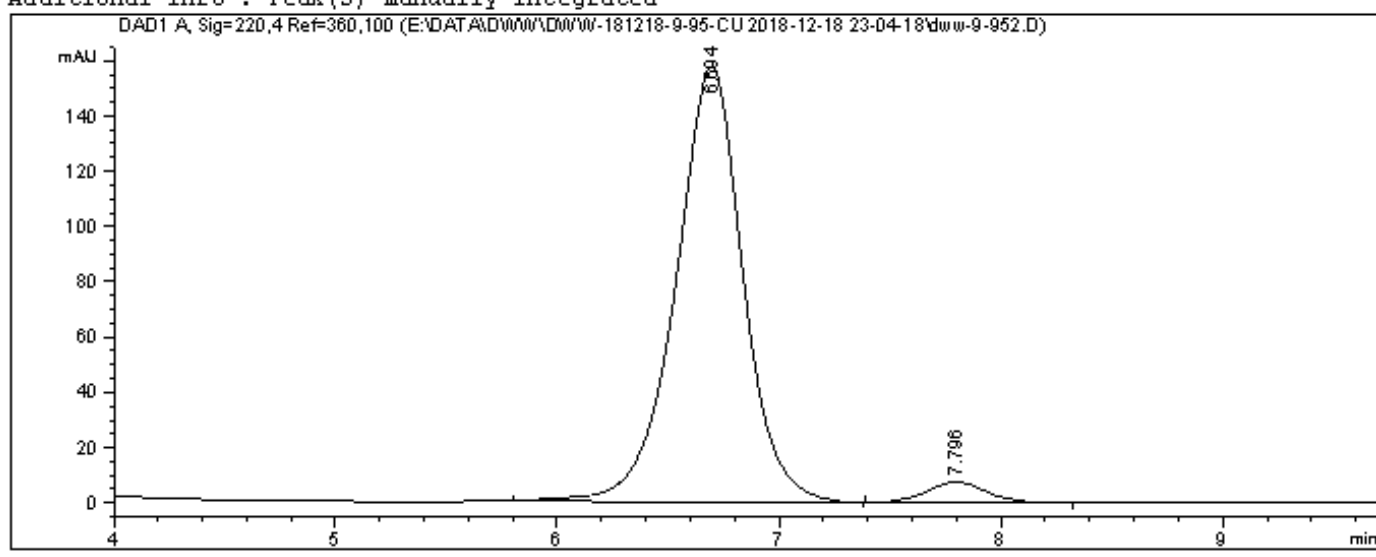

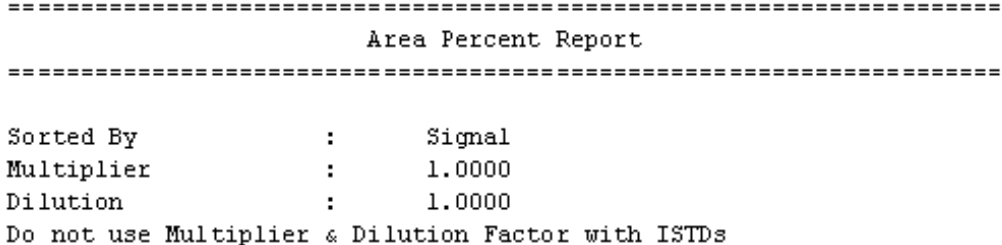

Signal 1: DẢDl À, Sig $=220,4$ Ref $=360,100$

\begin{tabular}{|c|c|c|c|c|c|}
\hline Peak & $\begin{array}{l}\text { RetTime Type } \\
\text { [min] }\end{array}$ & $\begin{array}{l}\text { Width } \\
\text { [min] }\end{array}$ & $\begin{array}{c}\text { Area } \\
\text { [mAUt*s] }\end{array}$ & $\begin{array}{l}\text { Height } \\
\text { [mAU] }\end{array}$ & Area \\
\hline-- & & ---1 & ------ & -- & -7 \\
\hline 1 & & & 3322 & 157 & 810 \\
\hline 2 & $7.796 \mathrm{BB}$ & .2340 & 142.73669 & 7.63950 & 4. 1190 \\
\hline
\end{tabular}

Totals :

$3465.36292 \quad 165.57944$

ネネー End of Report ネネ 
<smiles>CCC[C@H](CCc1ccccc1)CC(=O)OC</smiles>

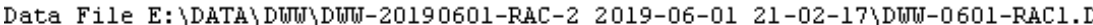
Sample Name: 1-Buene-RAC

\begin{tabular}{|c|c|c|c|}
\hline Acq. Operator & : SYSTEM & Seq. Line : & 2 \\
\hline Acq. Instrument & : 1260 & Location : & 46 \\
\hline Injection Date & : 6/1/2019 9:35:24 PM & Inj : & 1 \\
\hline
\end{tabular}

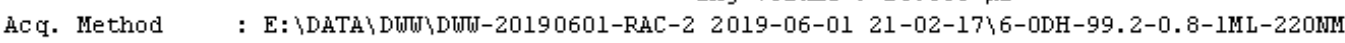
-30MIN-all-Tw0.M

Last changed : 6/1/2019 9:02:18 PM by SYSTEM

Analysis Method : E: \DATA $\backslash$ DUJЫ\DUJT-20190601-RAC-2 2019-06-01 21-02-17 6-0DH-99.2-0.8-1ML-220MM -30MIN-all-Two.M (Sequence Method)

Last changed : 7/24/2020 12:24:26 AM by SYSTEM (modified after loading)

Additional Info : Peak (s) manually integrated

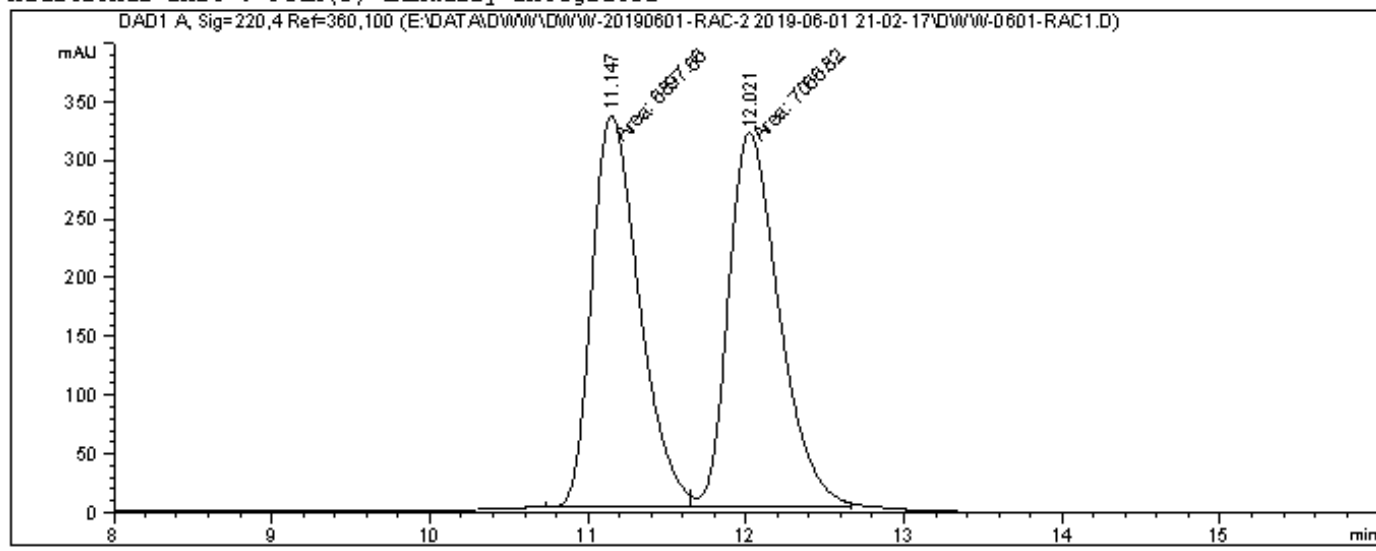

Area Percent Report

$\begin{array}{lll}\text { Sorted BY } & : & \text { Signal } \\ \text { Multiplier } & : & 1.0000 \\ \text { Dilution } & : & 1.0000\end{array}$

Do not use Multiplier \& Dilution Factor with IsTDs

Signal 1: DADl A, $S i g=220,4$ Ref $=360,100$

\begin{tabular}{|c|c|c|c|c|c|c|}
\hline $\begin{array}{c}\text { Peak } \\
\#\end{array}$ & $\begin{array}{c}\text { RetTime } \\
\text { [min] }\end{array}$ & Type & $\begin{array}{l}\text { Width } \\
\text { [min] }\end{array}$ & $\begin{array}{c}\text { Area } \\
{\left[\mathrm{mAU}^{*} \mathrm{~S}\right]}\end{array}$ & $\begin{array}{l}\text { Height } \\
\text { [miU] }\end{array}$ & $\begin{array}{c}\text { Area } \\
\text { के }\end{array}$ \\
\hline & & & & & & \\
\hline 1 & 11. & $1+1$ & 455 & 6897.66455 & 332.78506 & 49 \\
\hline 2 & 12.02 & FM & 0.3704 & 7066.81641 & 318.00278 & 50.6057 \\
\hline
\end{tabular}

Totals : $\quad 1.39645 \mathrm{e} 4 \quad 650.78784$ 


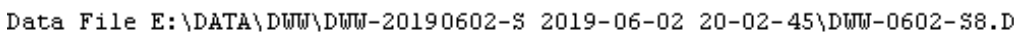
Sample Name: nPr-s

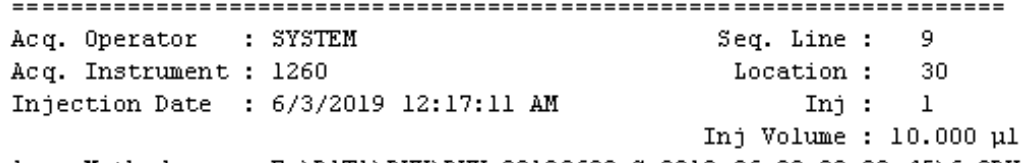

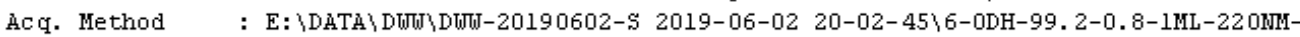
30MIN-all-TWO.M

Last changed : 6/2/2019 8:02:45 PM by SYSTEM

Analysis Method : E: \DATA DTW\DIJT-20190602-5 2019-06-02 20-02-45\6-0DH-99.2-0.8-1ML-22 0MM30MIN-all-Two.M (Sequence Method)

Last changed : 7/24/2020 12:33:51 AM by SYSTEM

(modified after loading)

Additional Info : Peak (s) manually integrated

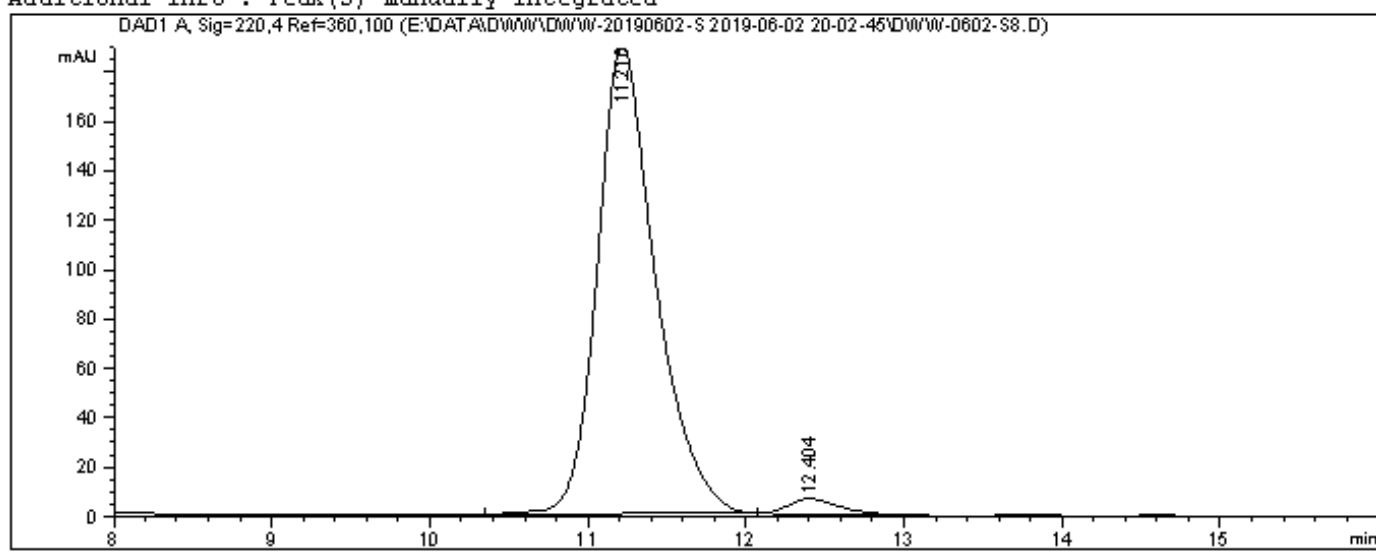

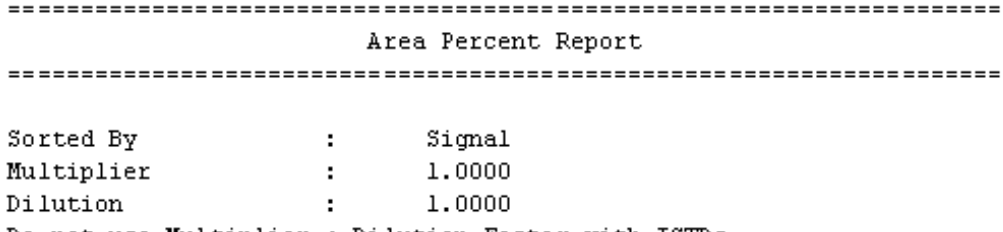

Do not use Multiplier \& Dilution Factor with ISTDs

Signal 1: DADl A, $S i g=220,4$ Ref $=360,100$

\begin{tabular}{|c|c|c|c|c|c|c|}
\hline $\begin{array}{c}\text { Peak } \\
\#\end{array}$ & $\begin{array}{c}\text { RetTime } \\
\text { [min] }\end{array}$ & Type & $\begin{array}{l}\text { Width } \\
\text { [min] }\end{array}$ & $\begin{array}{c}\text { Area } \\
{\left[\mathrm{m} \text { AU } U^{*} \mathrm{~S}\right]}\end{array}$ & $\begin{array}{l}\text { Height } \\
\text { [mAU] }\end{array}$ & $\begin{array}{c}\text { Area } \\
\stackrel{5}{*}\end{array}$ \\
\hline & & & & & & \\
\hline 1 & 11.2 & & & 4839. & 190. & 97 \\
\hline 2 & 12. & & 59 & 140.82451 & 6.13347 & 2.8275 \\
\hline
\end{tabular}

Totals : $\quad 4980.61748 \quad 196.32605$ 
<smiles>CCCC[C@H](CCc1ccccc1)CC(=O)OC</smiles>

Data File E: \DATA \DUT\DUJJ-20190601-RAC-2 2019-06-01 21-02-17\DUJJ-0601-RAC.D Sample Name: n-Bu-RAC

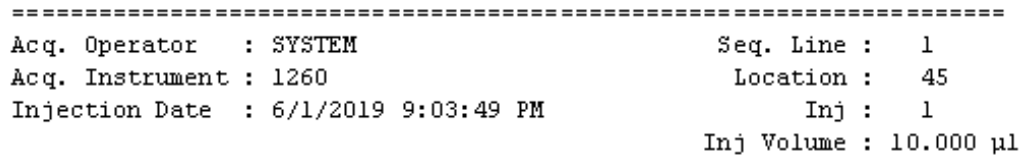

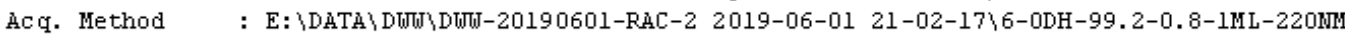
-30MIN-all-Tw0.M

Last changed : 6/1/2019 9:02:18 PM by SYSTEM

Analysis Method : E: \DATA $\backslash$ DUJЫ\DUJT-20190601-RAC-2 2019-06-01 21-02-17 6-0DH-99.2-0.8-1ML-220MM -30MIN-all-Two.M (Sequence Method)

Last changed : 7/24/2020 12:20:11 AM by SYSTEM (modified after loading)

Additional Info : Peak (s) manually integrated

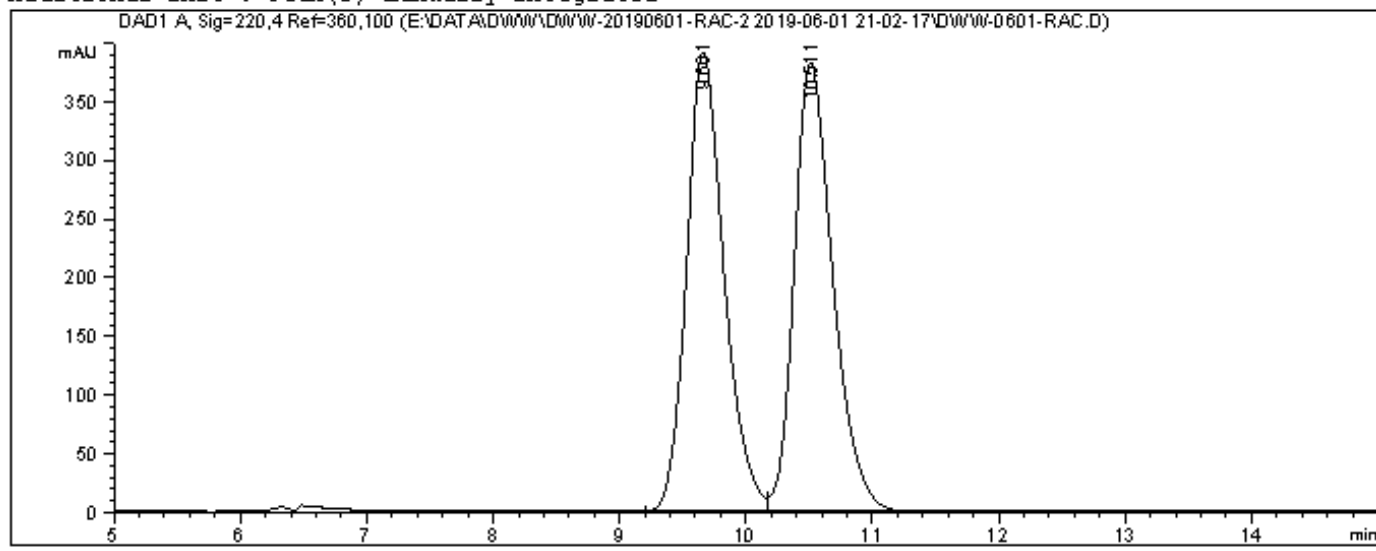

Area Percent Report

$\begin{array}{lll}\text { Sorted BY } & : & \text { Signal } \\ \text { Multiplier } & : & 1.0000 \\ \text { Dilution } & : & 1.0000\end{array}$

Do not use Multiplier \& Dilution Factor with IsTDs

Signal 1: DADl A, Sig $=220,4$ Ref $=360,100$

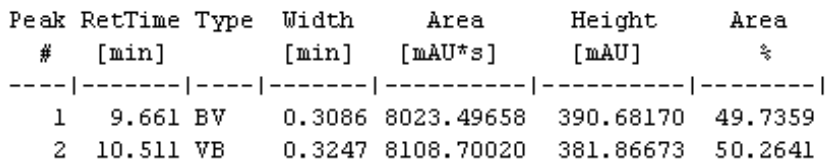

Totals : $\quad 1.61322 \mathrm{e} 4 \quad 772.54843$ 


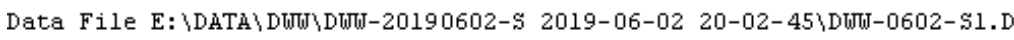
Sample Name: $n-B u-5$

\begin{tabular}{|c|c|c|c|}
\hline Acq. Operator & : SYSTEM & seq. Line : & 2 \\
\hline Acq. Instrument & : 1260 & Location : & 37 \\
\hline Injection Date & : 6/2/2019 8:36:00 PM & $\begin{array}{r}\text { Inj : } \\
\text { Inj Volume : }\end{array}$ & $\begin{array}{l}1 \\
0.000 \mu l\end{array}$ \\
\hline
\end{tabular}

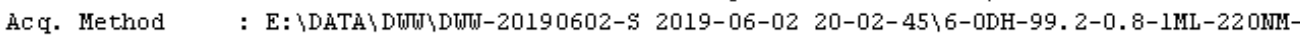
30MIN-al 1-TW0.M

Last changed : 6/2/2019 8:02:45 PM by SYSTEM

Analysis Method : E: \DATA DTW\DIJT-20190602-5 2019-06-02 20-02-45\6-0DH-99.2-0.8-1ML-22 0MM30MIN-all-Two.M (Sequence Method)

Last changed : 7/24/2020 12:38:36 AM by SYSTEM

(modified after loading)

Additional Info : Peak (s) manually integrated

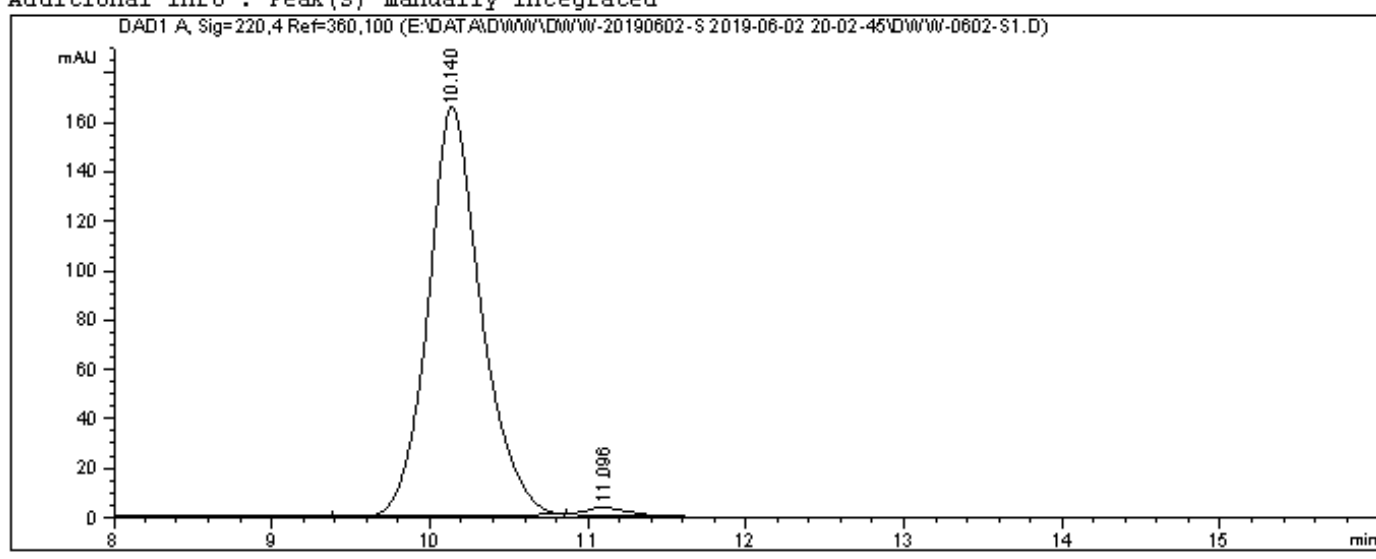

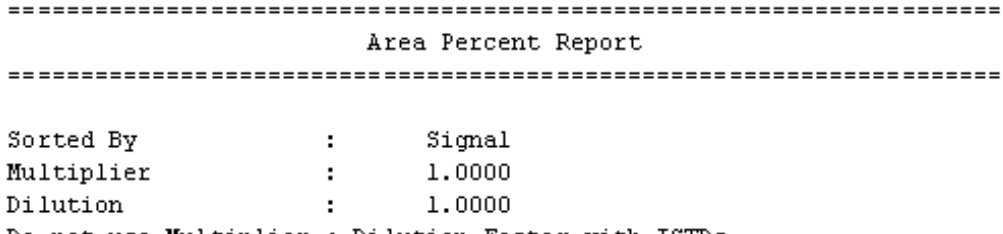

Do not use Multiplier \& Dilution Factor with ISTDs

Signal 1: DADl A, $S i g=220,4$ Ref $=360,100$

\begin{tabular}{|c|c|c|c|c|c|c|}
\hline $\begin{array}{c}\text { Peak } \\
\#\end{array}$ & $\begin{array}{c}\text { RetTime } \\
\text { [min] }\end{array}$ & Type & $\begin{array}{l}\text { Width } \\
\text { [min] }\end{array}$ & $\begin{array}{c}\text { Area } \\
{\left[\mathrm{mAU} \mathrm{U}^{*} \mathrm{~s}\right]}\end{array}$ & $\begin{array}{l}\text { Height } \\
\text { [mAU] }\end{array}$ & $\begin{array}{c}\text { Area } \\
\stackrel{5}{*}\end{array}$ \\
\hline- & & & & - & & \\
\hline 1 & & & & 3838 & 4034 & 624 \\
\hline 2 & 11.0 & $\mathrm{BB}$ & 52 & 4401 & 2.86196 & 1.3376 \\
\hline
\end{tabular}

Totals : $\quad 3890.95905 \quad 168.50229$

\#ネ End of Report ** 
<smiles>CCCCC[C@H](CCc1ccccc1)CC(=O)OC</smiles>

Data File E: \DATA \DUT\DUT-20190601-RAC-2 2019-06-01 21-02-17\DUJT-0601-RAC2.D Sample Name: n-Pent-RAC

$\begin{array}{llrl}\text { Acq. Operator } & : \text { SYSTEM } & \text { Seq. Line }: & 3 \\ \text { Acq. Instrument : } 1260 & \text { Location }: & 47 \\ \text { Injection Date } & : 6 / 1 / 2019 \quad 10: 07: 01 \mathrm{PM} & \text { Inj }: & 1 \\ & & \text { Inj Volume : } 10.000 \mu l\end{array}$

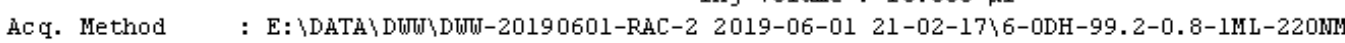
-30MIN-all-Tw0.M

Last changed : 6/1/2019 9:02:18 PM by SYSTEM

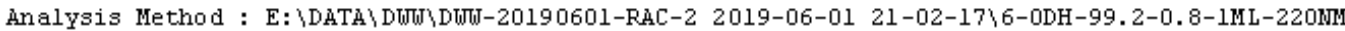
-30MIN-all-Two.M (Sequence Method)

Last changed : 7/24/2020 12:27:20 AM by SYSTEM

(modified after loading)

Additional Info : Peak (s) manually integrated

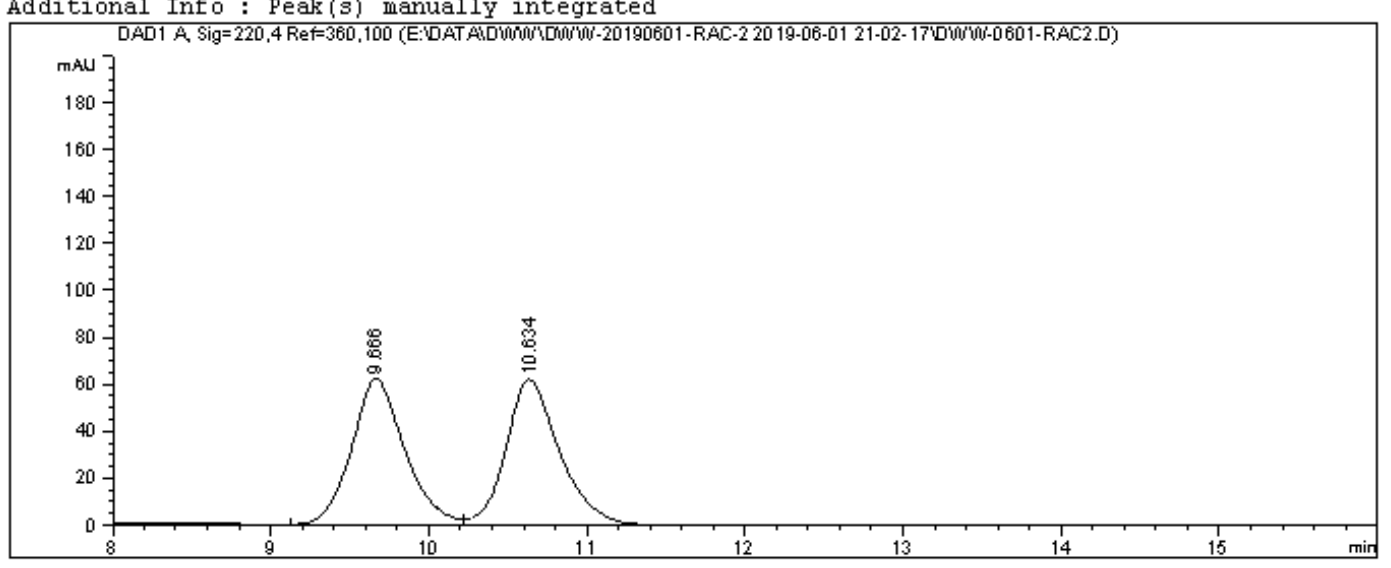

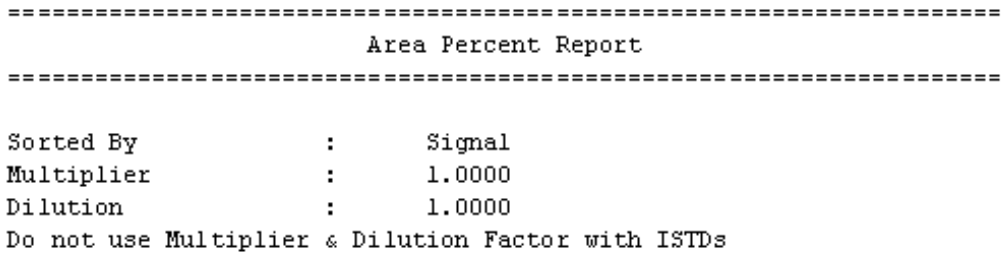

Signal 1: DADl A, Sig=220, 4 Ref $=360,100$

\begin{tabular}{|c|c|c|c|c|c|c|}
\hline $\begin{array}{c}\text { Peak } \\
\#\end{array}$ & $\begin{array}{c}\text { RetTime } \\
\text { [min] }\end{array}$ & Type & $\begin{array}{l}\text { Width } \\
\text { [min] }\end{array}$ & $\begin{array}{c}\text { Area } \\
{\left[\mathrm{m} \text { A } U^{*} \mathrm{*}\right]}\end{array}$ & $\begin{array}{l}\text { Height } \\
\text { [MAU] }\end{array}$ & $\begin{array}{c}\text { Area } \\
\stackrel{5}{5}\end{array}$ \\
\hline-- & - & & - & -- & ------ & 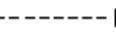 \\
\hline 1 & 9.666 & $\mathrm{BV}$ & 0.3420 & 1456.33228 & 62.23808 & 49.8183 \\
\hline 2 & 10.634 & VB & 0.3472 & 1466.95520 & 62.19436 & 50.1817 \\
\hline Total & : & & & 2923.28748 & 124.43243 & \\
\hline
\end{tabular}

ネネー End of Report 


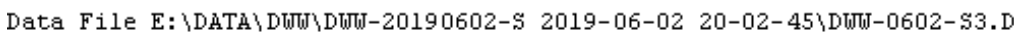
Sample Name: n-Pent-s
Acq. Operator : SYSTEM
seq. Line : 4
Ac q. Operator : SYSTE
Ac q. Instrument : 1260
Injection Date : 6/2/2019 9:39:15 PM
Location : 39
Inj : 1
Inj Volume : $10.000 \mu l$

Ac q. Method : E: \DATA \DUJJ 30MIN-all-TwO.M

Last changed : 6/2/2019 8:02:45 PM by SYSTEM

Analysis Method : E: \DATA D极\D财-20190602-S 2019-06-02 20-02-45 6-0DH-99.2-0.8-1ML-220MM30MIN-all-Two.M (Sequence Method)

Last changed : 7/24/2020 12:49:57 AM by SYSTEM

(modified after loading)

Additional Info: Peak (s) manually integrated

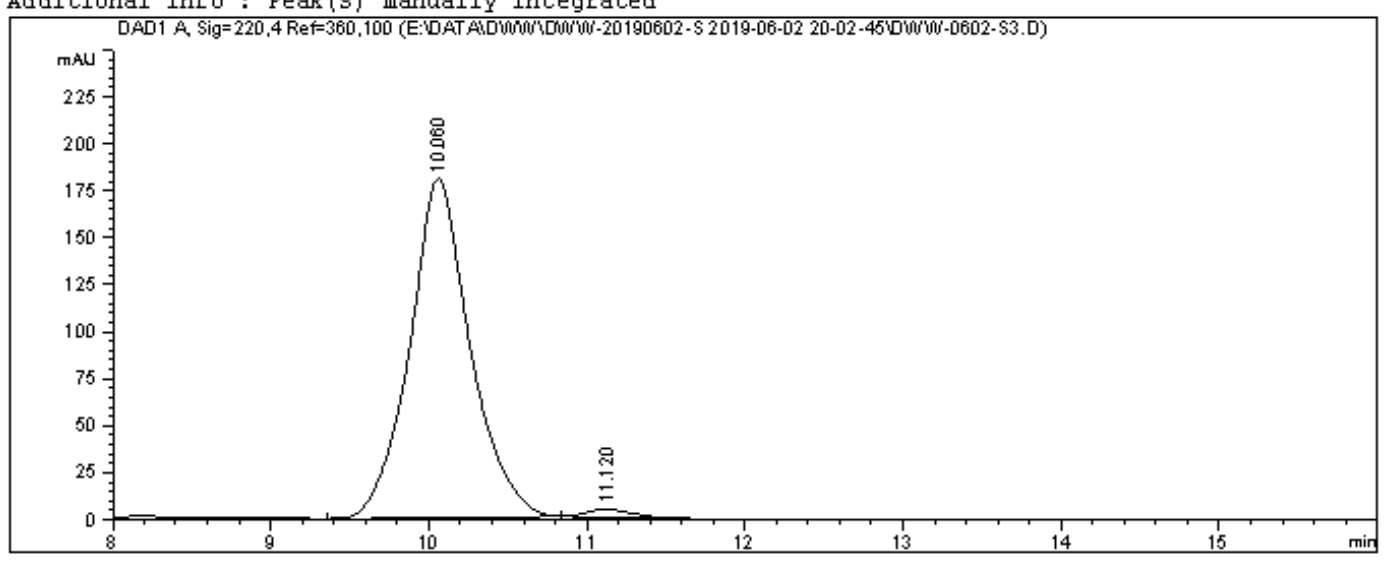

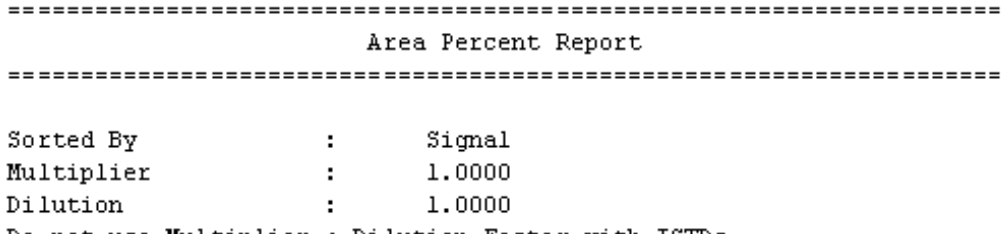

Do not use Multiplier \& Dilution Factor with ISTDs

Signal 1: DADl A, Sig $=220,4$ Ref $=360,100$

\begin{tabular}{|c|c|c|c|c|c|c|}
\hline $\begin{array}{c}\text { Peak } \\
\#\end{array}$ & $\begin{array}{c}\text { RetTime } \\
\text { [min] }\end{array}$ & Type & $\begin{array}{l}\text { Width } \\
\text { [min] }\end{array}$ & $\begin{array}{c}\text { Area } \\
{\left[\mathrm{m}^{2} \mathrm{H}^{*} \mathrm{~s}\right]}\end{array}$ & $\begin{array}{l}\text { Height } \\
\text { [mAU] }\end{array}$ & $\begin{array}{c}\text { Area } \\
\stackrel{5}{*}\end{array}$ \\
\hline- & & & & -- & & \\
\hline 1 & 10.0 & & & 4796 & 8745 & 794 \\
\hline 2 & 11.1 & $\mathrm{BB}$ & 0.2644 & 83.97083 & 4. 16248 & 1.7206 \\
\hline
\end{tabular}

Totals : $\quad 4880.41663 \quad 184.94993$

ネネ゙ End of Report 


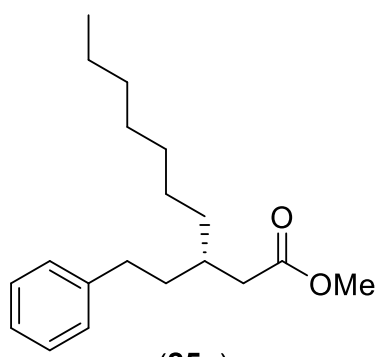

(25e)

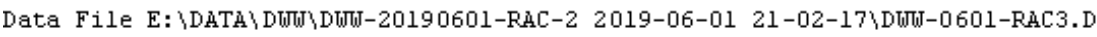
Sample Name: n-Hept-RAC

$\begin{array}{llrl}\text { Acq. Operator } & : \text { sYSTEM } & \text { Seq. Line : } & 4 \\ \text { Acq. Instrument : } 1260 & \text { Location : } & 48 \\ \text { Injection Date } & : 6 / 1 / 2019 \quad 10: 38: 41 \mathrm{PM} & \text { Inj }: & 1 \\ & & \text { Inj Volume : } 10.000 \mu 1\end{array}$

Acq. Method : E: \DATA \DWJ \UJ-20190601-RAC-2 2019-06-01 21-02-17 \6-0DH-99.2-0.8-1ML-220NM $-30 M I N-a l l-T$ To.M

Last changed : 6/1/2019 9:02:18 PM by SYSTEM

Analysis Method : E: \DATA DUT\\DJT-20190601-RAC-2 2019-06-01 21-02-17 6-0DH-99.2-0.8-1ML-220MM -30MIN-all-Two.M (Sequence Method)

Last changed : 7/24/2020 12:29:31 AM by SYSTEM

(modified after loading)

Additional Info : Peak (s) manually integrated

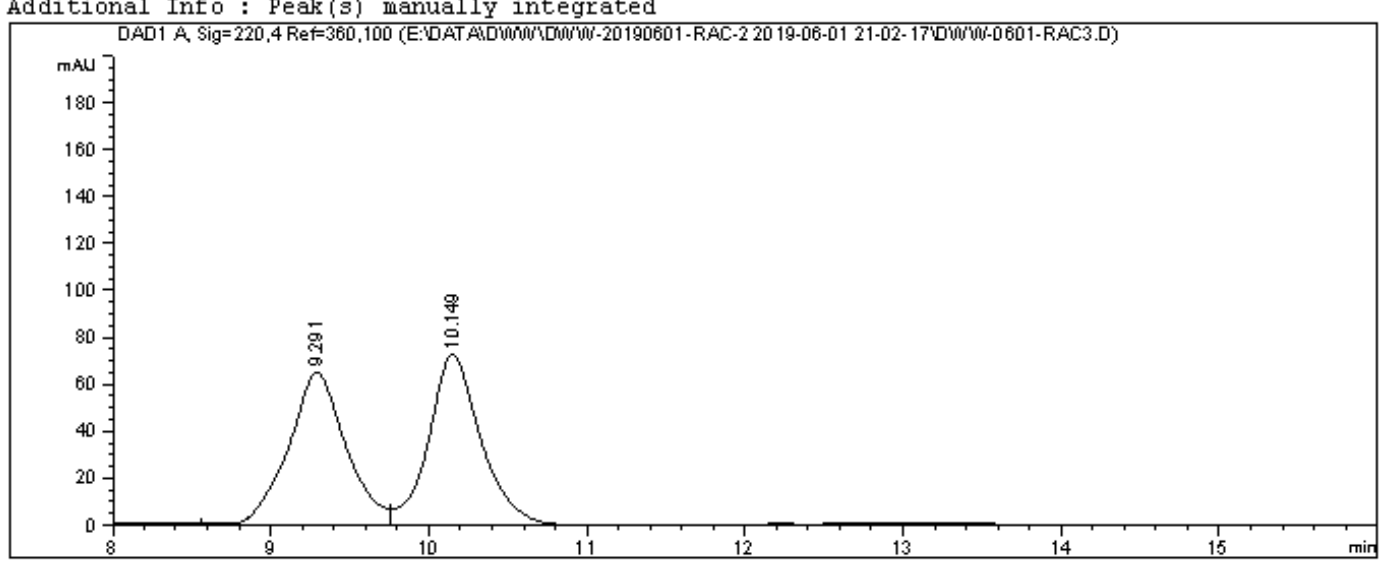

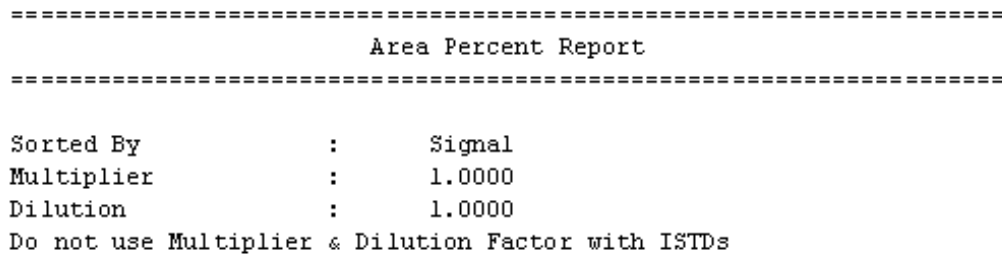

Signal 1: DADl A, Sig=220, 4 Ref $=360,100$

\begin{tabular}{|c|c|c|c|c|c|c|}
\hline $\begin{array}{c}\text { Peak } \\
\quad \#\end{array}$ & $\begin{array}{c}\text { RetTime } \\
\text { [min] }\end{array}$ & Type & $\begin{array}{l}\text { Width } \\
\text { [min] }\end{array}$ & 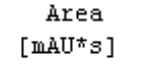 & $\begin{array}{l}\text { Height } \\
\text { [miU] }\end{array}$ & $\begin{array}{c}\text { Area } \\
\stackrel{5}{8}\end{array}$ \\
\hline & & & & -- & -- & \\
\hline 1 & 9.2 & & & 1639. & 64. & 49 \\
\hline 2 & 10.1 & B & 80 & 1654.57007 & 72.58106 & 50.2297 \\
\hline
\end{tabular}

Totals : $\quad 3294.00623 \quad 137.44532$

\#ネ End of Report 


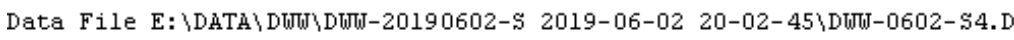
Sample Name: n-Hept-s
Acq. Operator : SYSTEM
Acq. Instrument : 1260
seq. Line : 5
Injection Date : 6/2/2019 10:10:54 PM
Location : 40
Inj : 1
Inj Volume : $10.000 \mu l$

Ac q. Method : E: \DATA \DUJJ 30MIN-al l-TWO.M

Last changed : 6/2/2019 8:02:45 PM by SYSTEM

Analysis Method : E: \DATA D极\D财-20190602-S 2019-06-02 20-02-45 6-0DH-99.2-0.8-1ML-220MM30MIN-all-Two.M (Sequence Method)

Last changed : 7/24/2020 12:54:49 AM by SYSTEM

(modified after loading)

Additional Info: Peak (s) manually integrated

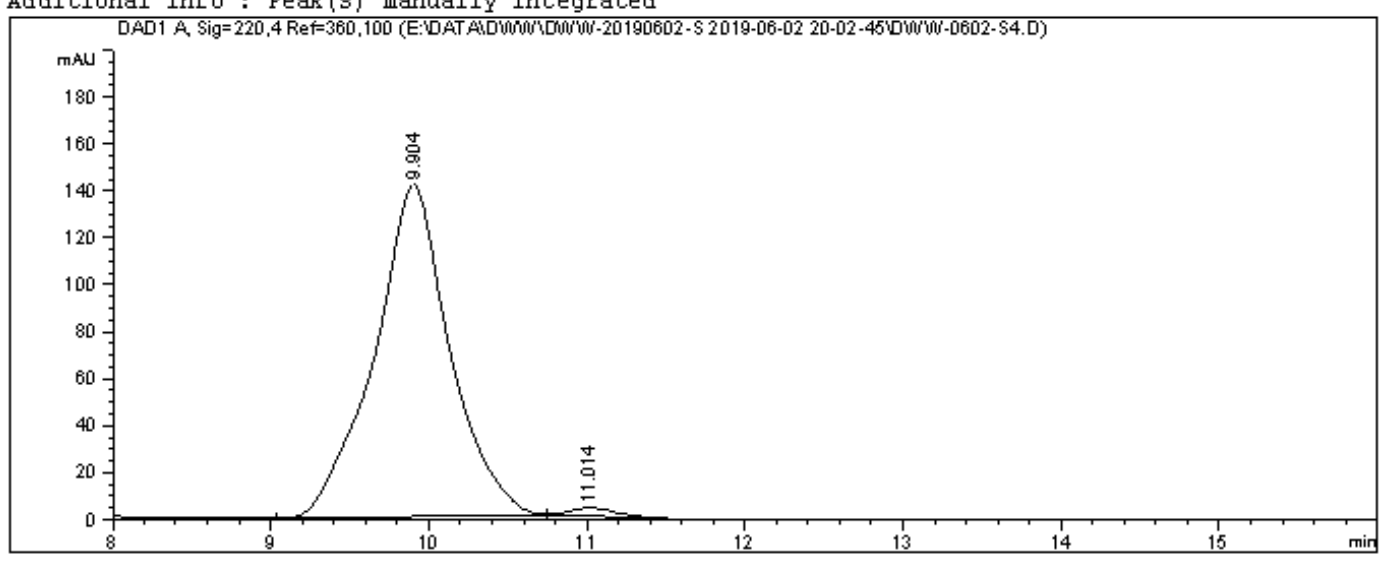

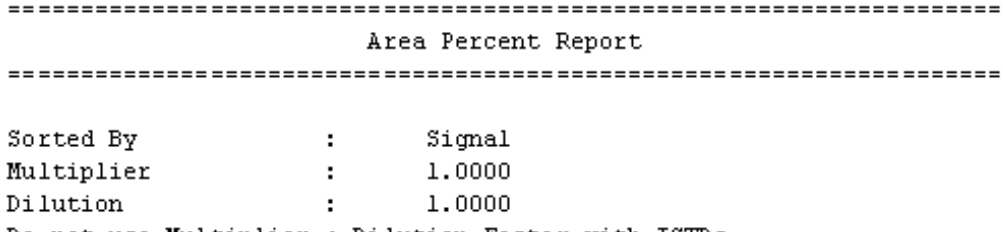

Do not use Multiplier \& Dilution Factor with ISTDs

Signal 1: DẢDl À, Sig $=220,4$ Ref $=360,100$

\begin{tabular}{|c|c|c|c|c|c|}
\hline $\begin{array}{c}\text { Peak } \\
\#\end{array}$ & $\begin{array}{l}\text { RetTime Type } \\
\text { [min] }\end{array}$ & $\begin{array}{l}\text { Width } \\
\text { [min] }\end{array}$ & $\begin{array}{c}\text { Area } \\
{\left[\mathrm{mAU}^{*} \mathrm{~S}\right]}\end{array}$ & $\begin{array}{l}\text { Height } \\
\text { [mAU] }\end{array}$ & $\begin{array}{c}\text { Area } \\
\frac{5}{5}\end{array}$ \\
\hline---1 & ------- & ------1 & --------- & $---------\mid$ & ----- \\
\hline 1 & $9.904 \mathrm{BB}$ & 0.4655 & 4686.75049 & 141.45494 & 98.4749 \\
\hline 2 & $11.014 \mathrm{BB}$ & 0.2922 & 72.58511 & 3.49690 & 1.5251 \\
\hline \multicolumn{3}{|c|}{ Totals : } & 4759.33559 & 144.95184 & \\
\hline
\end{tabular}

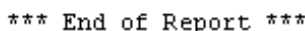


<smiles>COC(=O)C[C@H](C)CCc1ccccc1</smiles>

(25f)

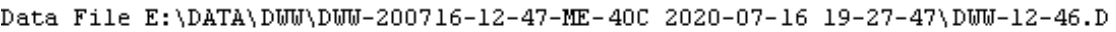
Sample Name: Doti-12-47-Me-rac

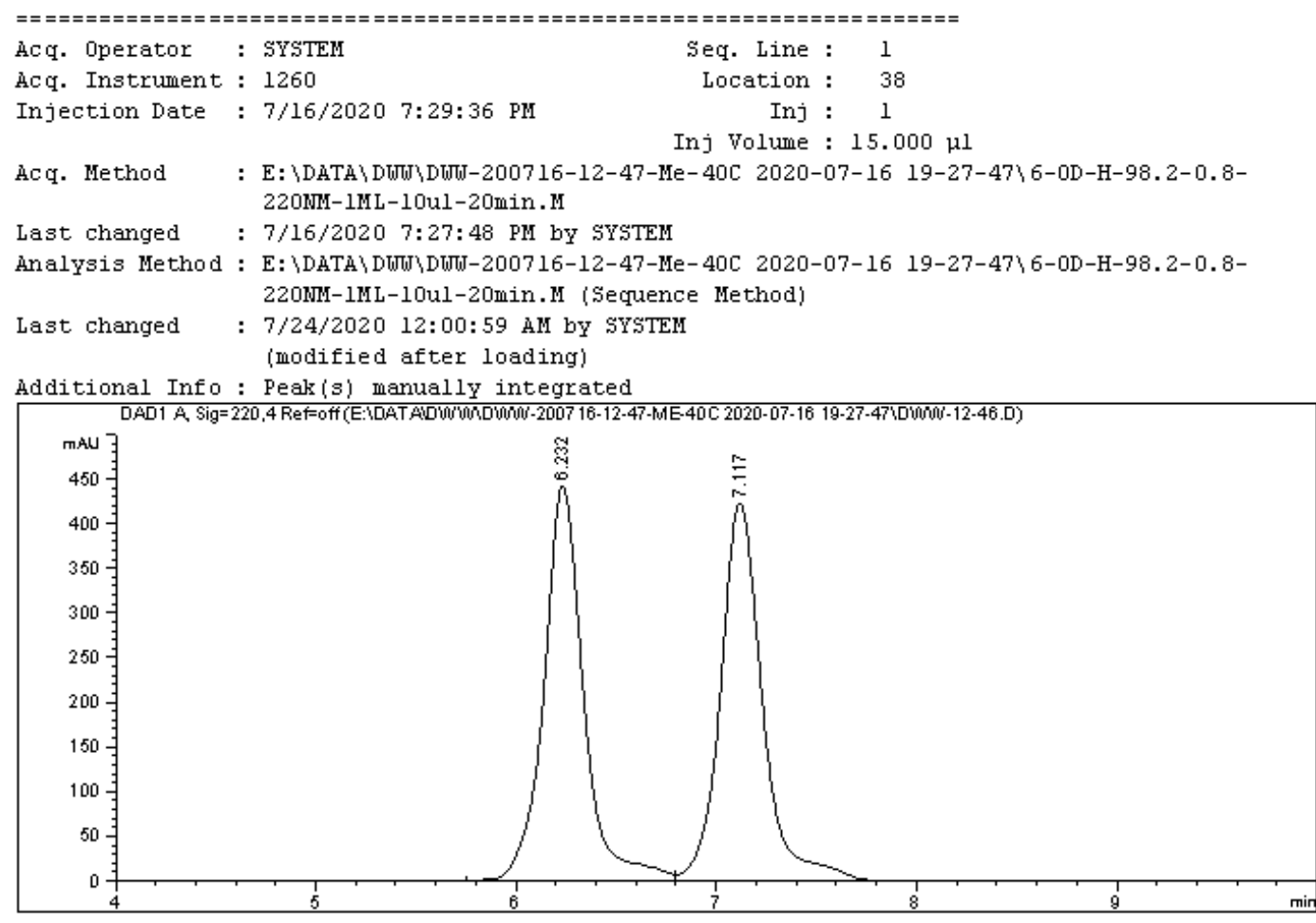

Area Percent Report

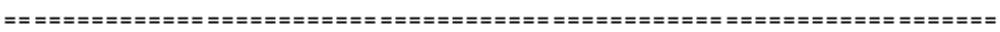

Sorted By : Signal

Multiplier : 1.0000

Dilution $\quad$ : 1.0000

Do not use Multiplier \& Dilution Factor with ISTDs

Signal 1: DADl A, Sig $=220,4$ Ref=off

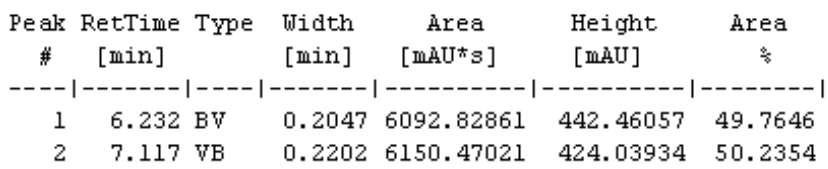

Totals : $\quad 1.22433 \mathrm{e} 4 \quad 866.49991$

ネネ End of Report *ネ 


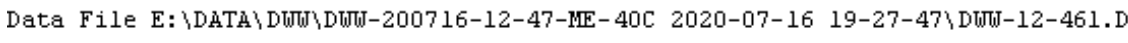
Sample Name: DoJj-12-47-Me-s
Acq. Operator : SYSTEM
Acq. Instrument : 1260
seq. Line : 2
Injection Date : 7/16/2020 7:51:11 PM
Location : 39
Inj : 1
Inj Volume : $15.000 \mu l$

Ac q. Method : E: \DATA $\backslash$ DUJW $\backslash$ DIJJ-200716-12-47-Me-40C 2020-07-16 19-27-47 6-0D-H-98.2-0.8220MM-1ML-10ul-20min. M

Last changed : 7/16/2020 7:27:48 PM by SYSTEM

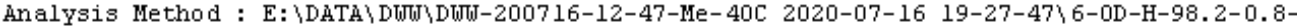
220MM-1ML-10ul-20min.M (Sequence Method)

Last changed : 7/24/2020 12:06:49 AM by SYSTEM

(modified after loading)

Additional Info : Peak (s) manually integrated

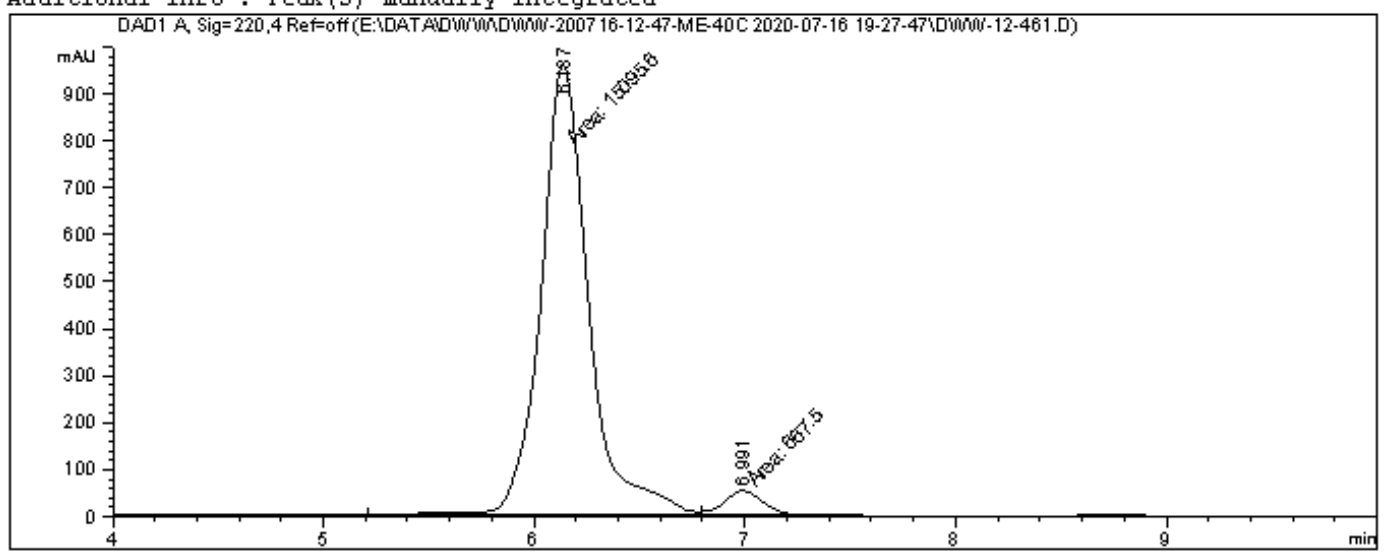

\section{Area Percent Report}

\begin{tabular}{|c|c|c|}
\hline Sorted By & : & Signal \\
\hline Multiplier & : & 1.0000 \\
\hline Dilution & : & 1.0000 \\
\hline
\end{tabular}

Do not use Multiplier \& Dilution Factor with ISTDs

Signal 1: DADl Â, Sig $=220,4$ Ref=off

\begin{tabular}{|c|c|c|c|c|c|}
\hline $\begin{array}{c}\text { Peak } \\
\#\end{array}$ & $\begin{array}{l}\text { RetTime Type } \\
\text { [min] }\end{array}$ & $\begin{array}{l}\text { Width } \\
\text { [min] }\end{array}$ & $\begin{array}{c}\text { Area } \\
{\left[\mathrm{mAU}^{*} \mathrm{~S}\right]}\end{array}$ & $\begin{array}{l}\text { Height } \\
\text { [mAU] }\end{array}$ & $\begin{array}{c}\text { Area } \\
\stackrel{5}{*}\end{array}$ \\
\hline---1 & $\mid------1$ & -----1 & $---------\mid$ & $---------\mid$ & ------1 \\
\hline 1 & $6.137 \mathrm{MF}$ & 0.2622 & $1.50956 \mathrm{e} 4$ & 959.50757 & 95.7654 \\
\hline 2 & $6.991 \mathrm{FM}$ & 0.2126 & 667.49969 & 52.31715 & 4.2346 \\
\hline Total & : & & $1.57631 \mathrm{e} 4$ & 1011.82472 & \\
\hline
\end{tabular}

ネネ* End of Report *ネ* 
<smiles>C=CCC[C@H](CCc1ccccc1)CC(=O)OC</smiles>

Data File E: \DATA \DUJ \DाJ-20190601-RAC-2 2019-06-01 21-02-17\DUJJ-0601-RACl.D Sample Name: 1-Buene-RAC

\begin{tabular}{|c|c|c|c|}
\hline Acq. Operator & : SYSTEM & Seq. Line : & 2 \\
\hline Acq. Instrument & : 1260 & Location : & 46 \\
\hline Injection Date & : 6/1/2019 9:35:24 PM & Inj : & 1 \\
\hline
\end{tabular}

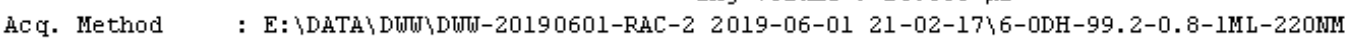
-30MIN-all-Tw0.M

Last changed : 6/1/2019 9:02:18 PM by SYSTEM

Analysis Method : E: \DATA $\backslash$ DUJЫ\DUJT-20190601-RAC-2 2019-06-01 21-02-17 6-0DH-99.2-0.8-1ML-220MM -30MIN-all-Two.M (Sequence Method)

Last changed : 7/24/2020 12:24:26 AM by SYSTEM (modified after loading)

Additional Info : Peak (s) manually integrated

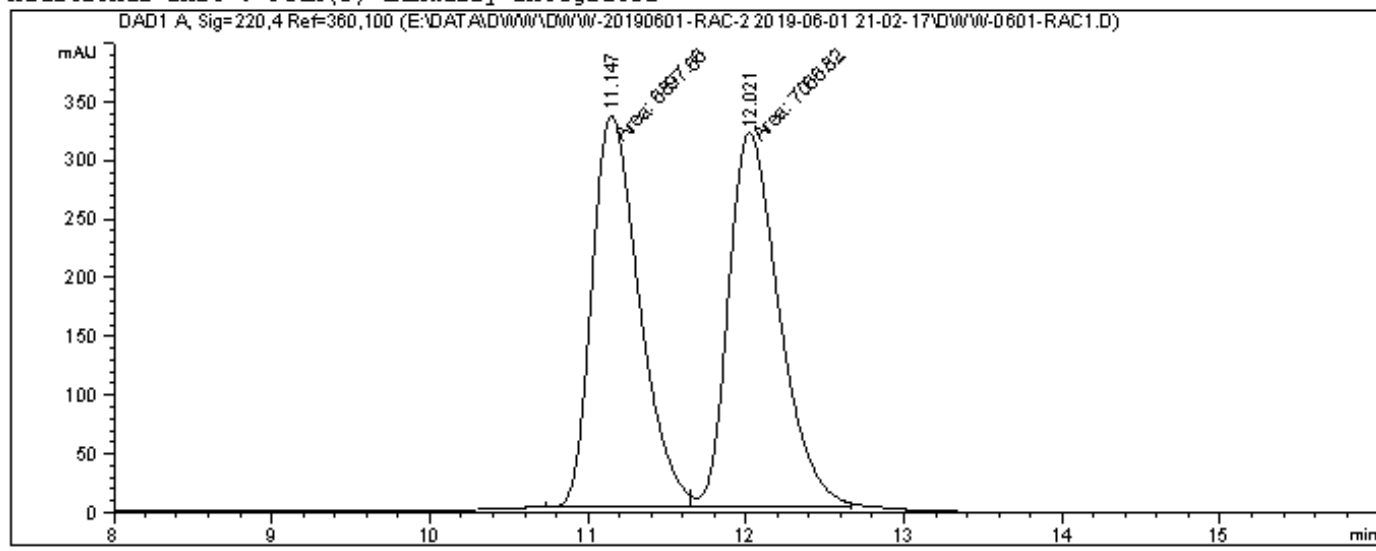

Àrea Percent Report

$\begin{array}{lll}\text { Sorted BY } & : & \text { Signal } \\ \text { Multiplier } & : & 1.0000 \\ \text { Dilution } & : & 1.0000\end{array}$

Do not use Multiplier \& Dilution Factor with IsTDs

Signal 1: DADl A, $S i g=220,4$ Ref $=360,100$

\begin{tabular}{|c|c|c|c|c|c|c|}
\hline $\begin{array}{c}\text { Peak } \\
\#\end{array}$ & $\begin{array}{c}\text { RetTime } \\
\text { [min] }\end{array}$ & Type & $\begin{array}{l}\text { Width } \\
\text { [min] }\end{array}$ & $\begin{array}{c}\text { Area } \\
{\left[\mathrm{mAU}^{*} \mathrm{~S}\right]}\end{array}$ & $\begin{array}{l}\text { Height } \\
\text { [miU] }\end{array}$ & $\begin{array}{c}\text { Area } \\
\text { के }\end{array}$ \\
\hline & & & & & & \\
\hline 1 & 11. & $1+1$ & 455 & 6897.66455 & 332.78506 & 49 \\
\hline 2 & 12.02 & FM & 0.3704 & 7066.81641 & 318.00278 & 50.6057 \\
\hline
\end{tabular}

Totals : $\quad 1.39645 \mathrm{e} 4 \quad 650.78784$ 
Data File E: \DATA $\backslash$ DाJЫ Sample Name: 1-Buene-S
Acq. Operator : SYSTEM
seq. Line : 3
Ac q. Operator : SYSTE
Ac q. Instrument : 1260
Injection Date : 6/2/2019 9:07:36 PM
Location : 38
Inj : 1
Inj Volume : $10.000 \mu l$

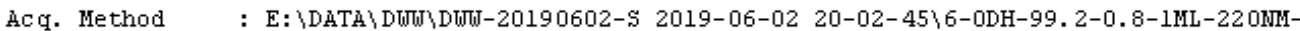
30MIN-all-TW0.M

Last changed : 6/2/2019 8:02:45 PM by SYSTEM

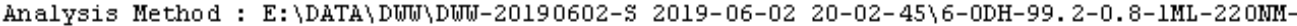
30MIN-all-Two.M (Sequence Method)

Last changed : 7/24/2020 12:46:45 AM by SYSTEM

(modified after loading)

Additional Info : Peak (s) manually integrated

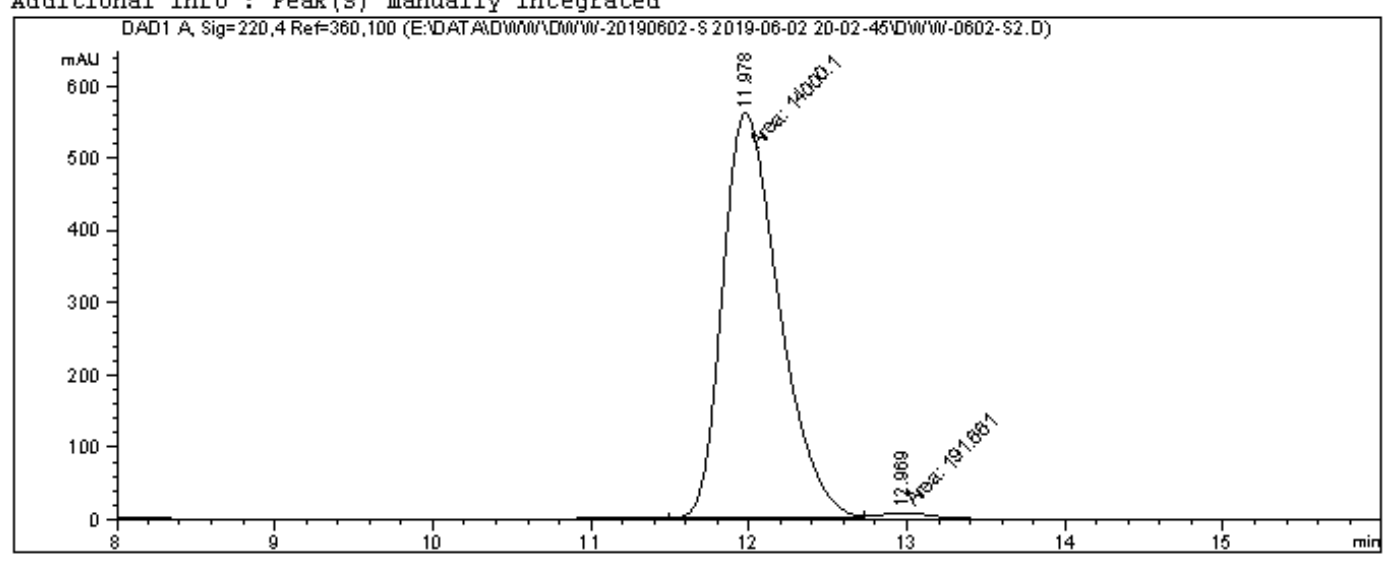

Area Percent Report

\begin{tabular}{|c|c|c|}
\hline Sorted BY & : & Signal \\
\hline Multiplier & : & 1.0000 \\
\hline Dilution & : & 1.0000 \\
\hline
\end{tabular}

Do not use Multiplier \& Dilution Factor with ISTDs

Signal 1: DADl A, Sig $=220,4$ Ref $=360,100$

\begin{tabular}{|c|c|c|c|c|c|c|}
\hline $\begin{array}{c}\text { Peak } \\
\#\end{array}$ & $\begin{array}{c}\text { RetTime } \\
\text { [min] }\end{array}$ & Type & $\begin{array}{l}\text { Width } \\
\text { [min] }\end{array}$ & $\begin{array}{c}\text { Area } \\
{\left[\mathrm{m} \text { AU } U^{*} \mathrm{~S}\right]}\end{array}$ & $\begin{array}{l}\text { Height } \\
\text { [mAU] }\end{array}$ & $\begin{array}{c}\text { Area } \\
\frac{0}{*}\end{array}$ \\
\hline & & & & & & \\
\hline 1 & 1 & & 0 & 1.400 & 560.84442 & 495 \\
\hline 2 & 12.9 & & 725 & 6104 & 8.57614 & L. 3505 \\
\hline
\end{tabular}

Totals : $\quad 1.41918 \mathrm{e} 4 \quad 569.42056$

\#ネ End of Report ** 
<smiles>CCOC(=O)C[C@H](C)CC</smiles>

(25h)

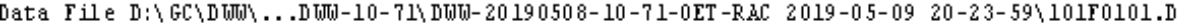
Sample Irame: Dow-10-71-0Et-rac

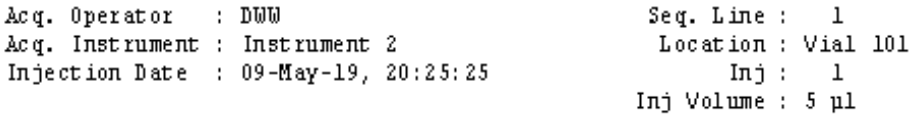

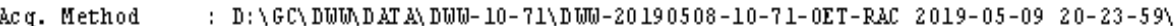
CH IRALDEX - GTA-60C-1ML-60MII.

Last charged : 4/11/2019 10:18:42 MM bY CC

Aлalys is Method : D: \GC LYM MET HOD BETA-DEX-390-80C-90M III-140C-30M IIN-19ML .M

Last changed : 5/2/2030 4:52:59 M bY LY

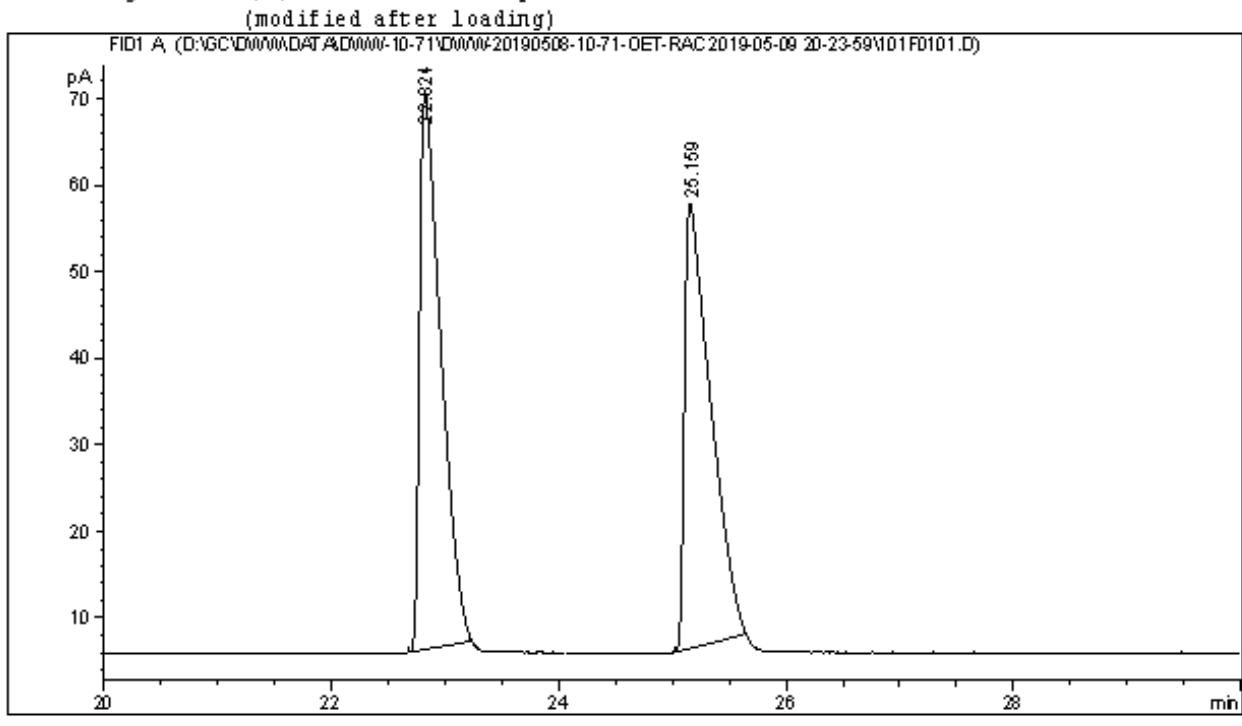

Area Percent Report

\begin{tabular}{|c|c|c|c|c|}
\hline Sorted BY & : & Signal & & \\
\hline Mult iplier & : & 1.0000 & & \\
\hline Dilution & $:$ & 1.0000 & & \\
\hline \multicolumn{2}{|c|}{ Use Multiplier \& Dilution } & Factor with & ISTD s & \\
\hline \multicolumn{5}{|l|}{ Signal $1:$ FID $1 \mathrm{~A}$, } \\
\hline $\begin{array}{l}\text { Peak RetT ime TYpe } \\
\# \quad \text { [miл] }\end{array}$ & $\begin{array}{l}\text { Width } \\
\text { [mir] }\end{array}$ & $\begin{array}{r}\text { hrea } \\
{\left[\mathrm{ph}^{*} \mathrm{~s}\right]}\end{array}$ & $\begin{array}{l}\text { Height } \\
\text { [pa] }\end{array}$ & $\begin{array}{c}\text { Area } \\
\text { 몸 }\end{array}$ \\
\hline$---|------|---1$ & $|------|$ & $|-------|$ & -------1 & $|-------|$ \\
\hline $122.824 \mathrm{BB}$ & 0.1725 & 837.44788 & 64.06317 & 50.67851 \\
\hline $2 \quad 25.159 \mathrm{BB}$ & 0.1894 & 815.02356 & 51.28515 & 49.32149 \\
\hline Totals : & & 1652.47144 & 115.34832 & \\
\hline
\end{tabular}

त* End of Report 


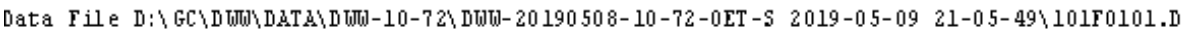
Sample IIame: Dtw-10-72-0Et-guarg
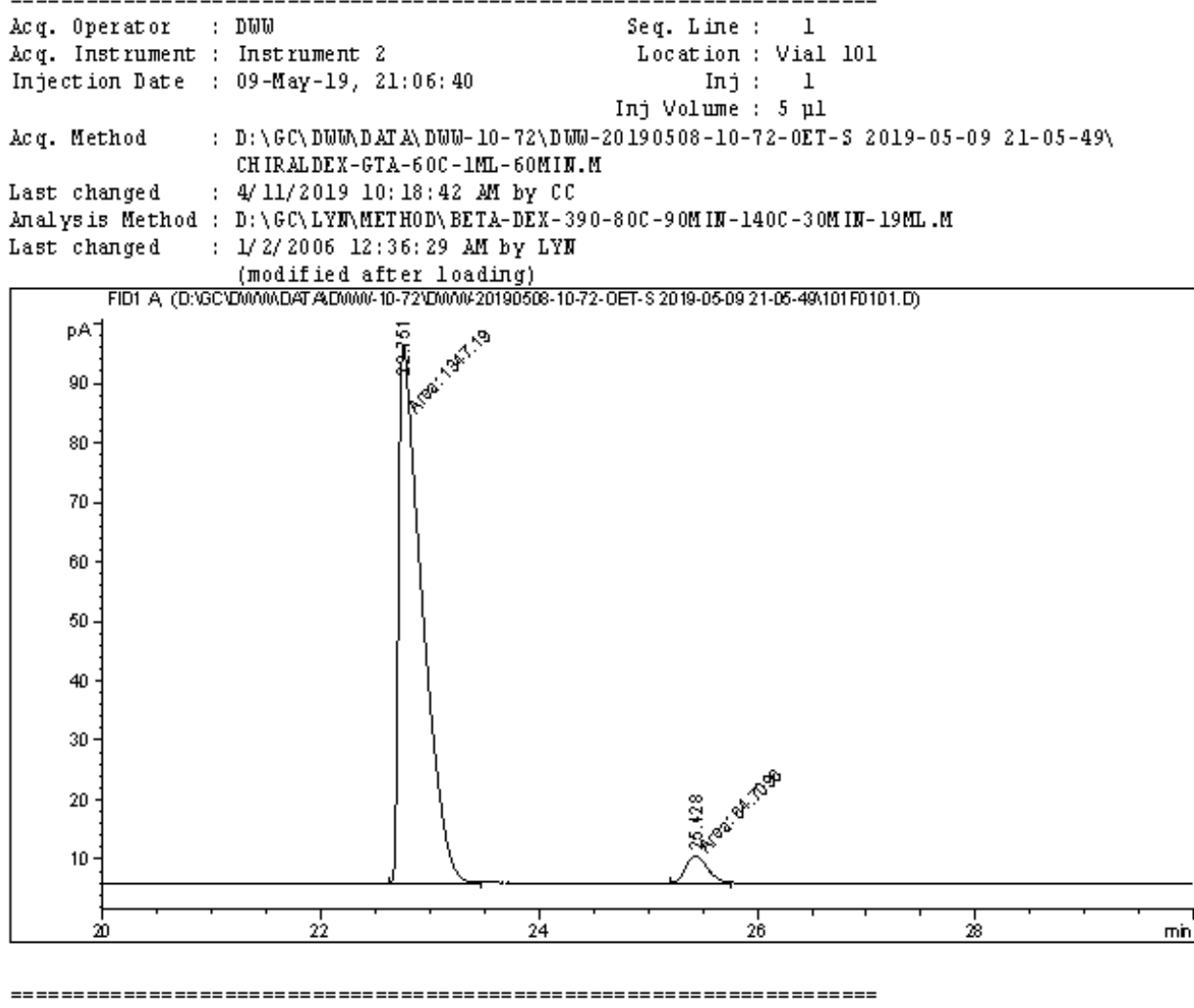

Area Percent Report

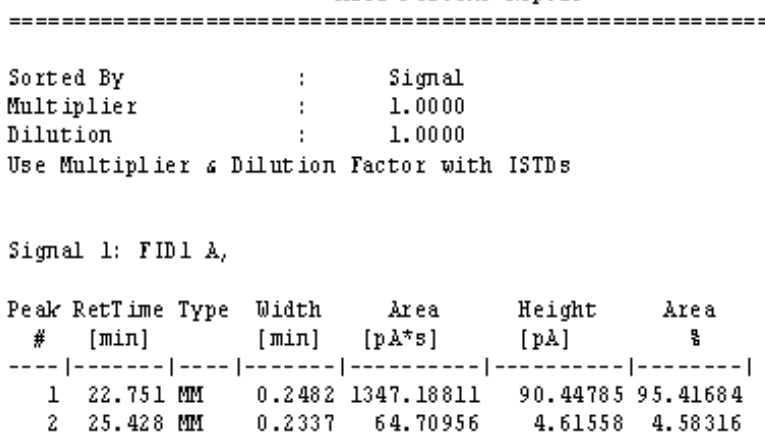

Totals : $\quad 1411.89767 \quad 95.06343$

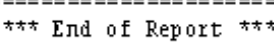


<smiles>CCCC(CC)CC(=O)OC</smiles>

(25i)

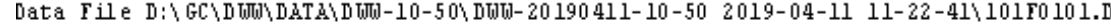
Sample Irame: ת-P

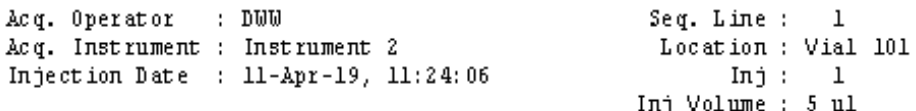

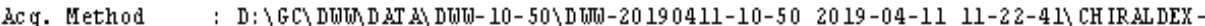
GT A-60C- 1ML - 6 OM III.M

Last charged : 4/ $11 / 201910: 18: 42$ M by CC

Aralys is Method : D: \GC LYM MET H0D BETA-DEX-390-80C-90M III-140C-30M IN- 19ML .M

Last charged : 5/2/2030 5:05:05 MM bY LYM

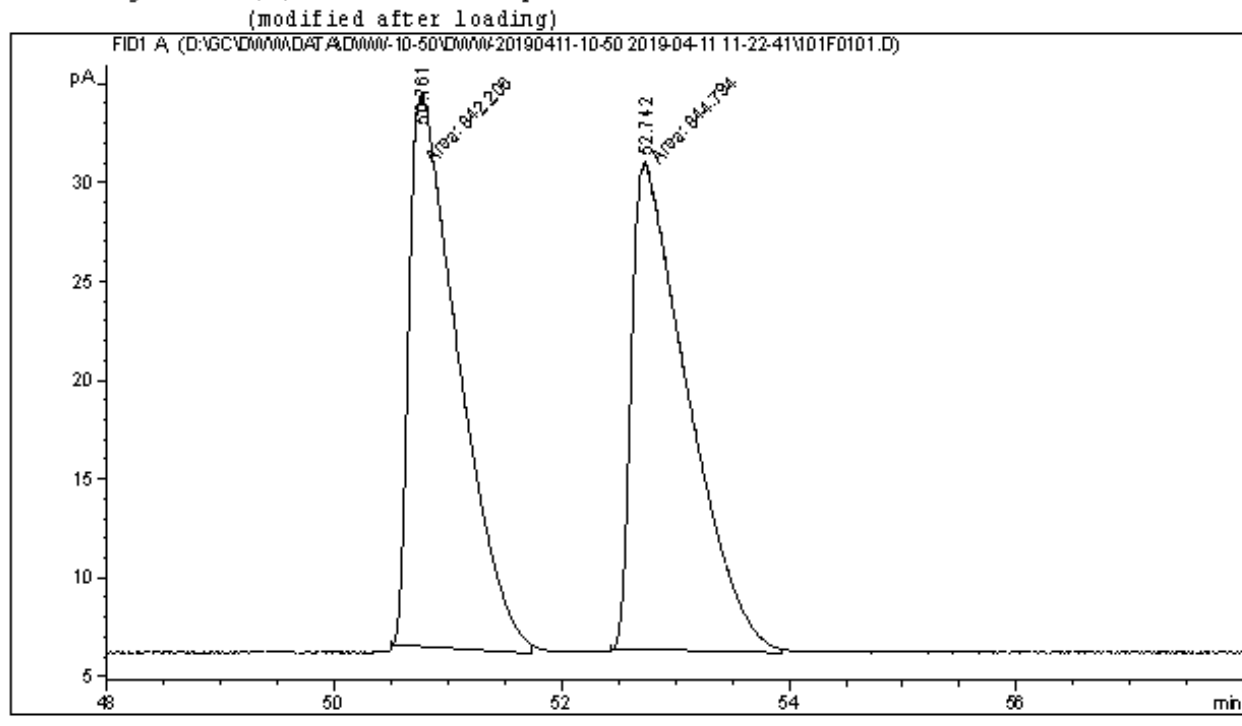

Area Percent Report

\begin{tabular}{|c|c|c|}
\hline Sorted BY & : & Signal \\
\hline Mult iplier & $:$ & 1.0000 \\
\hline Dilution & : & 1.0000 \\
\hline
\end{tabular}

Use Multiplier $\&$ Dilution Factor with ISTDs

Signal l: $\mathrm{FID} \perp \mathrm{A}$,

\begin{tabular}{|c|c|c|c|c|c|}
\hline $\begin{array}{c}\text { Peak } \\
\#\end{array}$ & $\begin{array}{l}\text { RetT ime TYpe } \\
\text { [miл] }\end{array}$ & $\begin{array}{l}\text { Width } \\
\text { [miת] }\end{array}$ & $\begin{array}{r}\text { hrea } \\
{\left[p A^{*} s\right]}\end{array}$ & $\begin{array}{l}\text { Height } \\
{[\mathrm{pA}]}\end{array}$ & $\begin{array}{c}\text { Area } \\
\text { 몹 }\end{array}$ \\
\hline 1 & $50.761 \mathrm{MM}$ & 0.5013 & 842,20557 & 99966 & 49.92329 \\
\hline 2 & $52.742 \mathrm{MM}$ & 0.5700 & 844.79364 & 24.70115 & $\begin{array}{l}49.96369 \\
50.07671\end{array}$ \\
\hline rotal & & & 1686.99921 & 52.70081 & \\
\hline
\end{tabular}

校 End of Report 


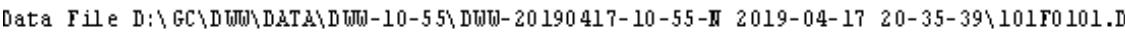
Sample II ame: Dtw- 10-55-II

\begin{tabular}{|c|c|c|}
\hline ac q. Operat or & : DWW & Seq. Line : \\
\hline ac q. Inst rument & : Inst rument 2 & Location: Vial 101 \\
\hline Irjection Date & $: 17-\operatorname{Apr}-19,20: 36: 41$ & 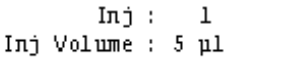 \\
\hline
\end{tabular}

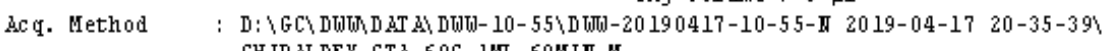
CH IRALDEX-GTA-60C-1ML-60MIII. N

Last changed : 4/11/2019 10:18:42 3 by CC

aralys is Method : D : \GC LYI MET HOD BETA-DEX-390-80C-90M III-140C-30M III- 19ML .M

Last charged : 5/2/2030 5:13:08 MM bY L YW
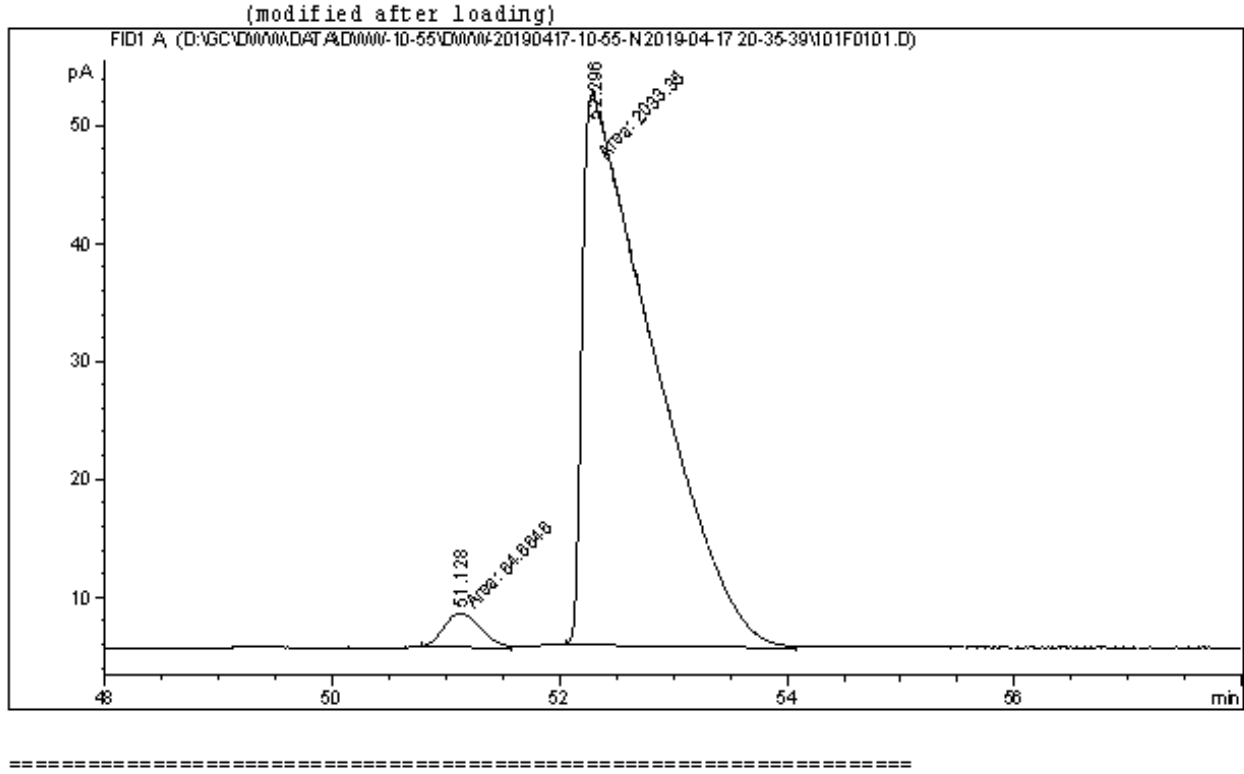

Area Percent Report

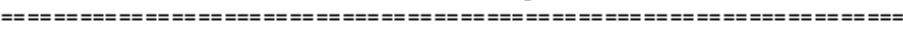

$\begin{array}{lll}\text { Sorted By } & : & \text { Signal } \\ \text { Multiplier } & : & 1.0000\end{array}$

Dilution : 1.0000

Use Multiplier s Dilution Factor with ISTDs

Signal l: FID 1 ,

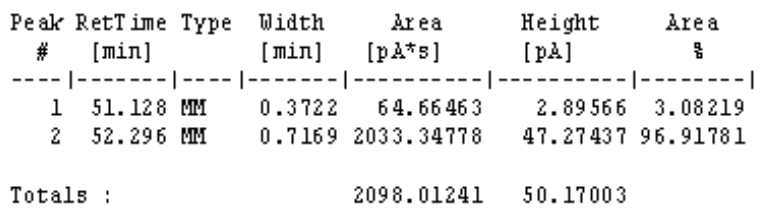

蔆 Erd of Report 
<smiles>CCC[C@H](CC)CC(=O)OCC</smiles>

$(25 \mathrm{j})$

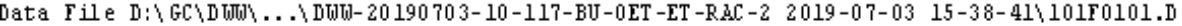
Sample Irame: $\pi-\mathrm{Bu}-0 \mathrm{Et}-\mathrm{Et}-\mathrm{rac}-2$

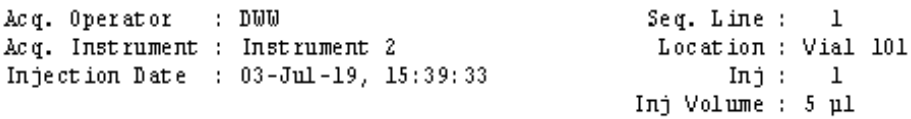

Ac q. Method : D : \GC DTWD D aT A DWT-10-117 DWW-20190703-10-117-BU-0ET-ET-RAC-2 2019-07-03 15-38-41 CH IR AL DEX-GTA-7 OC - 1ML- 12 OM II .M

Last charged : $7 / 3 / 20193: 34: 44$ PM bY DJW

Aralys is Method : D: \GC LYM MET H0D BETA-DEX-390-80C-90M IN-140C-30M IN- 19ML .M

Last charged : $5 / 2 / 2030 \quad 6: 27: 13$ HM bY LYT

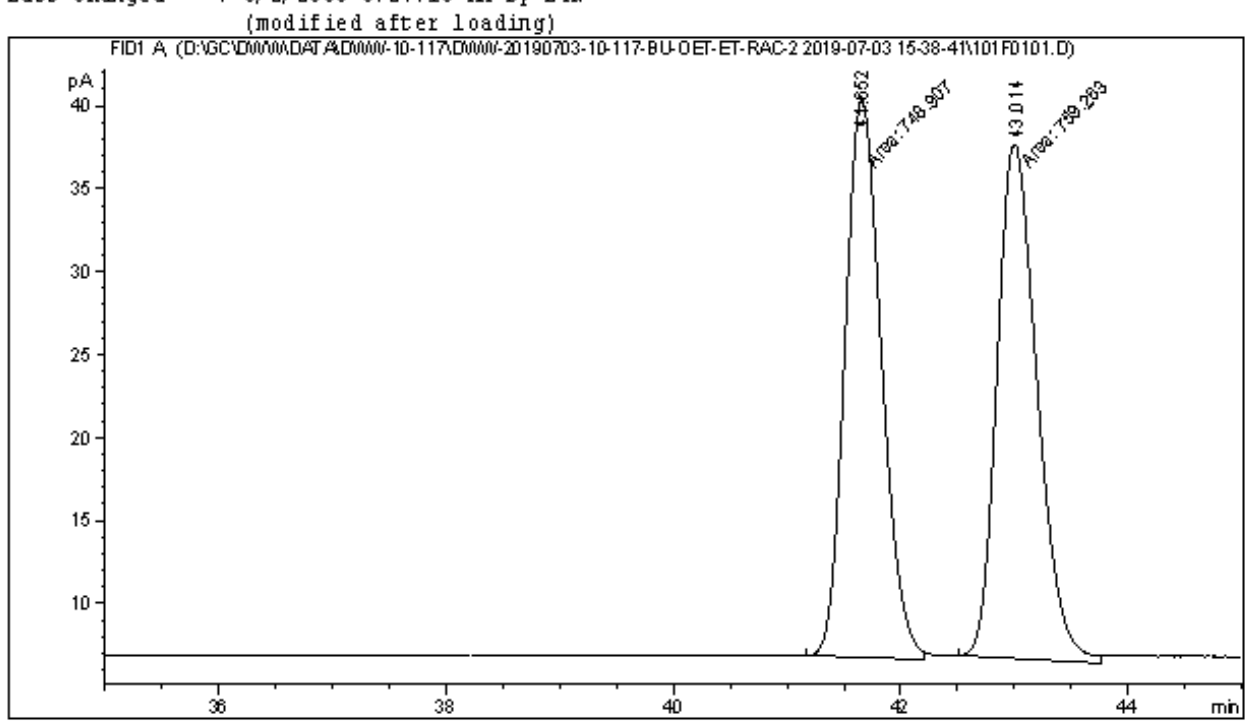

Area Percent Report

\begin{tabular}{|c|c|c|}
\hline Sorted BY & $:$ & Signal \\
\hline Mult iplier & $:$ & 1.0000 \\
\hline Dilution & : & 1.0000 \\
\hline
\end{tabular}

Jse Multiplier \& Dilution Factor with ISTDs

Signal 1: FID $1 \mathrm{~A}$

\begin{tabular}{|c|c|c|c|c|c|}
\hline $\begin{array}{c}\text { Peak } \\
\#\end{array}$ & $\begin{array}{l}\text { RetT ime TYpe } \\
\text { [miл] }\end{array}$ & $\begin{array}{l}\text { Width } \\
\text { [min] }\end{array}$ & $\begin{array}{r}\text { Area } \\
{\left[\mathrm{p}^{*} \mathrm{~s}\right]}\end{array}$ & $\begin{array}{l}\text { Height } \\
\text { [pA] }\end{array}$ & $\begin{array}{c}\text { Area } \\
\text { 몸 }\end{array}$ \\
\hline 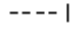 & 1 & & & & r \\
\hline 1 & $41.652 \mathrm{MM}$ & 0.3695 & 748.90747 & 33.78203 & 49.65670 \\
\hline 2 & $43.014 \mathrm{MM}$ & 0.4082 & 759.26257 & 31.00362 & 50.34330 \\
\hline ot & & & 1508.17004 & 64.78564 & \\
\hline
\end{tabular}

蔆 End of Report 


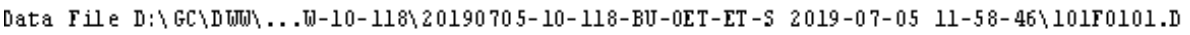
Sample Iame: rBu-OEt-Et-S

\begin{tabular}{|c|c|c|}
\hline Acq. Operator & : DWW & Seq. L іле : \\
\hline Ac q. Inst rument & : Inst rument 2 & Location: Vial 101 \\
\hline Irjection Date & : 05-Jul-19, 12:00:12 & $\begin{array}{rlc}\text { Iлj : } & & 1 \\
\text { Iлj Volume } & 5 & \text { pl }\end{array}$ \\
\hline
\end{tabular}

Ac q. Method : D: $\backslash G C \backslash D D W D A T A D D T-10-118 \backslash 20190705-10-118-B U-0 E T-E T-S \quad 2019-07-05 \quad 11-58-46 \backslash$ CH IRALDEX-GTA-70C-1ML-60MII.

Aral ys is Method : D : GC LYM MET HOD BETA-DEX-390-80C-90M III-140C-30M IN- 19ML .M

Last charged : $1 / 2 / 200612: 42: 42$ AM bY LYI
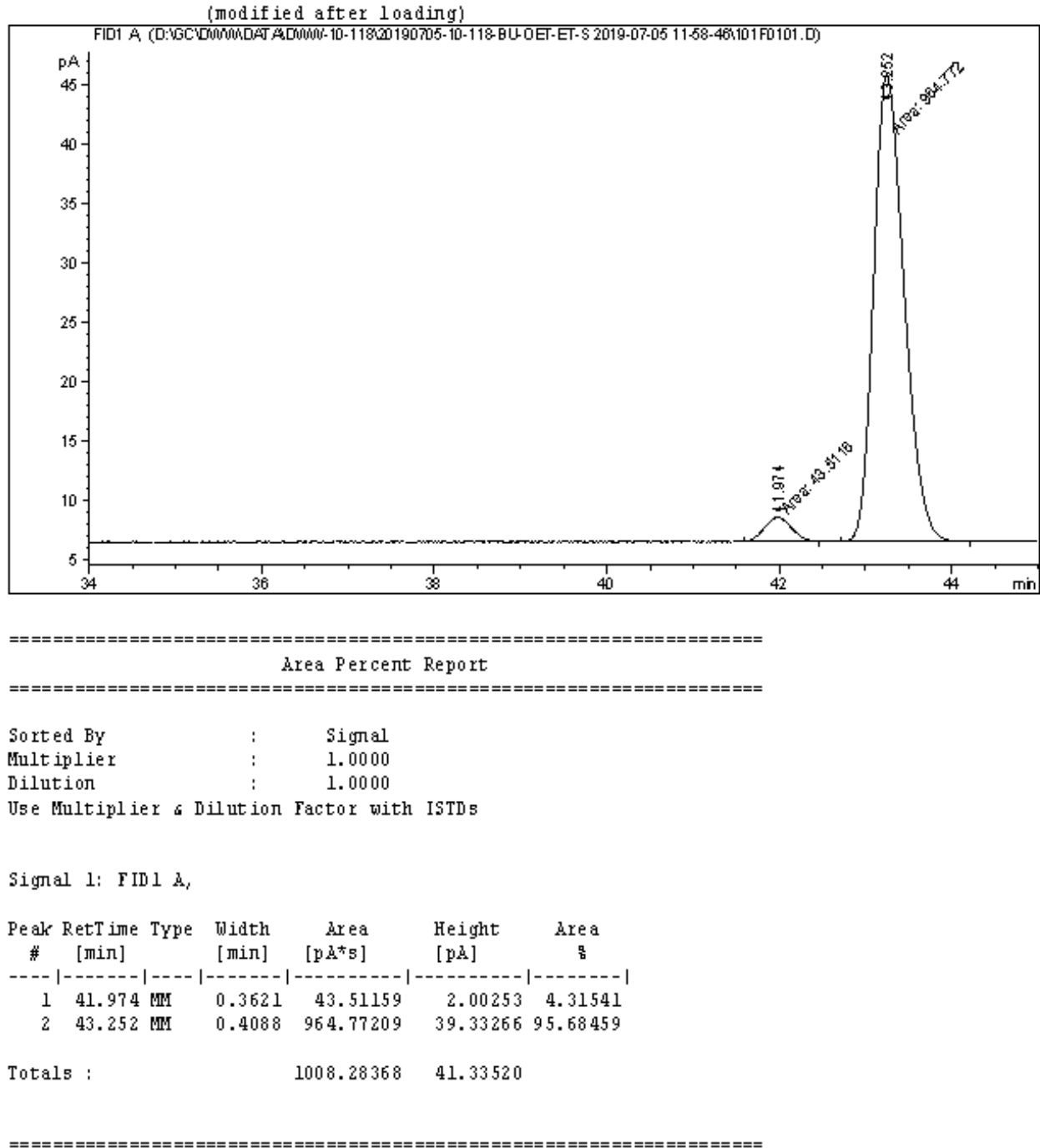

茫 End of Report 


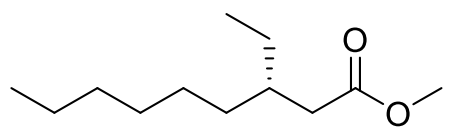

$(25 k)$

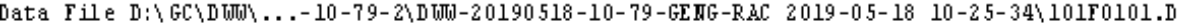
Sample Irame: Dtw-10-79-GEIG-RAC

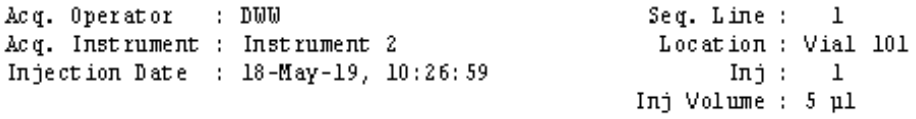

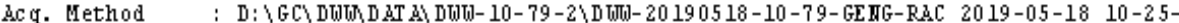
34\CHIRALDEX-GT A-90C-1ML-120MII .M

Last charged : 5/ 18/2019 10:25:23 M by DJTा

Aлalys is Method : D: \GC LYM MET H0D BETA-DEX-390-80C-90M III-140C-30M IIN-19ML .M

Last changed : 5/2/2030 6:21:34 MM bY L YT

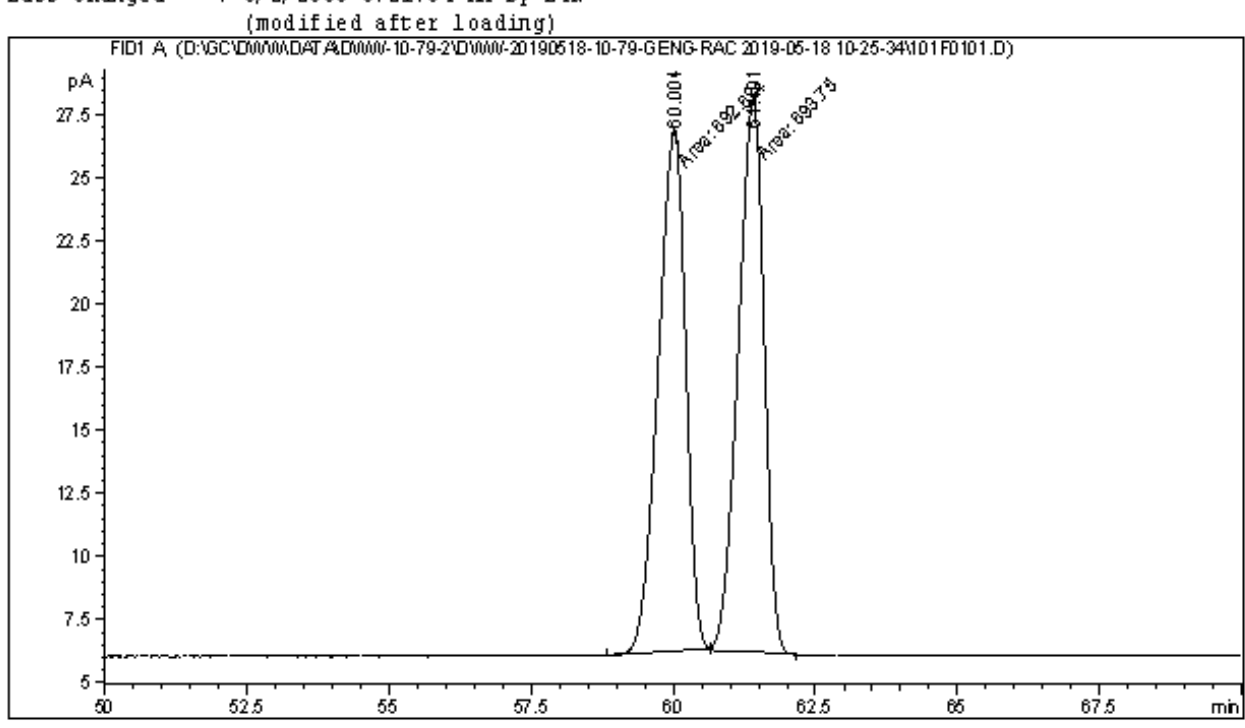

Area Percent Report

\begin{tabular}{|c|c|c|}
\hline Sorted By & : & Signal \\
\hline Mult iplier & : & 1.0000 \\
\hline Dilution & : & 1.0000 \\
\hline
\end{tabular}

Use Multiplier a Dilution Factor with ISTDs

Signal l: FIDI A,

\begin{tabular}{|c|c|c|c|c|c|}
\hline $\begin{array}{c}\text { Peak } \\
\#\end{array}$ & $\begin{array}{l}\text { RetT ime TYpe } \\
\text { [miл] }\end{array}$ & $\begin{array}{l}\text { Width } \\
\text { [min] }\end{array}$ & $\begin{array}{r}\text { hrea } \\
{\left[\mathrm{p}^{*} \mathrm{~s}\right]}\end{array}$ & $\begin{array}{l}\text { Height } \\
\text { [pA] }\end{array}$ & $\begin{array}{c}\text { Area } \\
\text { 몹 }\end{array}$ \\
\hline 1 & $60.004 \mathrm{MM}$ & 0.5566 & 692.62378 & 73797 & $\mid---1---1$ \\
\hline$\frac{1}{2}$ & $61.401 \mathrm{MM}$ & 0.5257 & 693.74951 & 21.99477 & 50.04060 \\
\hline ota. & & & 1386.37329 & 42.73275 & \\
\hline
\end{tabular}

校 End of Report 


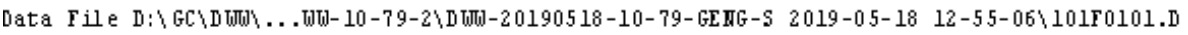
Sample IIame: DDT-10-79-GEIG-GUAIRG

\begin{tabular}{|c|c|c|}
\hline acq. Operat or & : DTW & Seq. Line : \\
\hline ac q. Inst rument & : Inst rument 2 & Location: Vial 101 \\
\hline Injection Date & : 18-MaY-19, 12:56:09 & $\begin{array}{rlll}\text { Iлј : } & & 1 \\
\text { Irj Volume } & 5 & \text { Hl }\end{array}$ \\
\hline
\end{tabular}

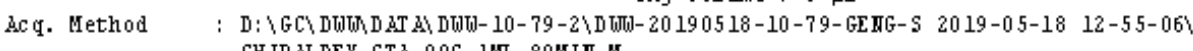
CH IR ALDEX-GTA-90C-1ML-80MIN. M

Last changed : 5/18/2019 12:54:19 PM by DiJi

Aralys is Method : D : \GC LYI MET HOD BETA-DEX-390-80C-90M III-140C-30M III- 19ML .M

Last changed : 5/2/2030 6:23:54 HM bY LY (modified after loading)

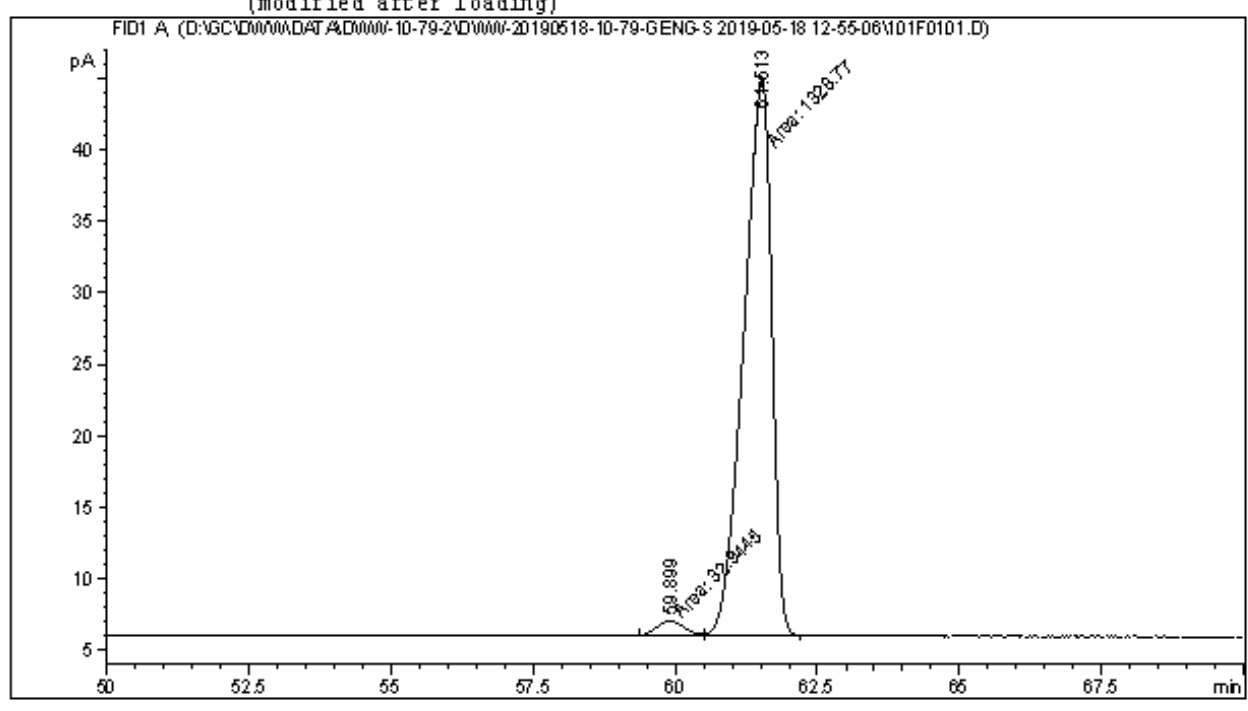

Area Percent Report

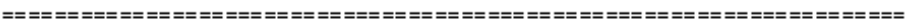

$\begin{array}{lll}\text { Sorted BY } & : & \text { Signal } \\ \text { Multiplier } & : & 1.0000 \\ \text { Dillution } & : & 1.0000\end{array}$

Dilution

1.0000

Use Multiplier s Dilution Factor with ISTDs

Signal l: FID 1 ,

\begin{tabular}{|c|c|c|c|c|c|c|}
\hline $\begin{array}{c}\text { Peak } \\
\#\end{array}$ & $\begin{array}{l}\text { RetT ime } \\
\text { [miл] }\end{array}$ & TYpe & $\begin{array}{l}\text { Tidth } \\
\text { [mis] }\end{array}$ & $\begin{array}{r}\text { hrea } \\
{\left[p A^{*} s\right]}\end{array}$ & $\begin{array}{l}\text { Height } \\
{[\mathrm{pA}]}\end{array}$ & $\begin{array}{c}\text { Area } \\
\text { 몸 }\end{array}$ \\
\hline- & $--\cdots$ & -7 & 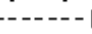 & ----- & -- & --1 \\
\hline 1 & 59.899 & MF & 0.5582 & 32.94452 & $9.83741 \mathrm{e}-1$ & 2.41934 \\
\hline 2 & 61.513 & $\mathrm{M}$ & 0.5683 & 1328.76819 & 38.96585 & 97.58066 \\
\hline ot & & & & 1361.71271 & 39.94959 & \\
\hline
\end{tabular}

离萑 Erd of Report 


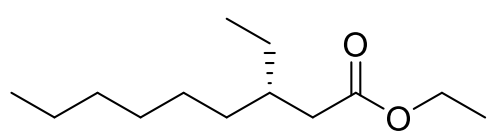

(25I)

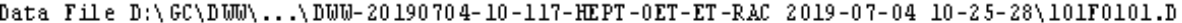
Sample Irame: Hept-0Et-Et-rac

\begin{tabular}{|c|c|c|}
\hline Acq. Operat or & : DדाW & Seq. L іле : \\
\hline Ac q. Inst rument & : Iлst rument 2 & Location: Vial 101 \\
\hline Irjection Date & : 04-Jul-19, 10:26:50 & $\begin{array}{rlc}\text { Iлj : } & & 1 \\
\text { Iлj Volume } & 5 & \text { pl }\end{array}$ \\
\hline
\end{tabular}

Ac q. Method : D : \GC DTWD D AT A DWW-10-117 DWW-20190704-10-117-HEPT-0ET-ET-RAC 2019-07-04 $10-25-28 \backslash$ CH IR ALDEX - GT A-80C - 1ML-210M II .M

Last charged : $7 / 4 / 201910: 20: 38$ AM bY Diti

Aлalys is Method : D: \GC LYM MET H0D BETA-DEX-390-80C-90M IIN-140C-30M IN- 19ML . M

Last charged : 5/2/2030 6:31:52 MM bY L YM

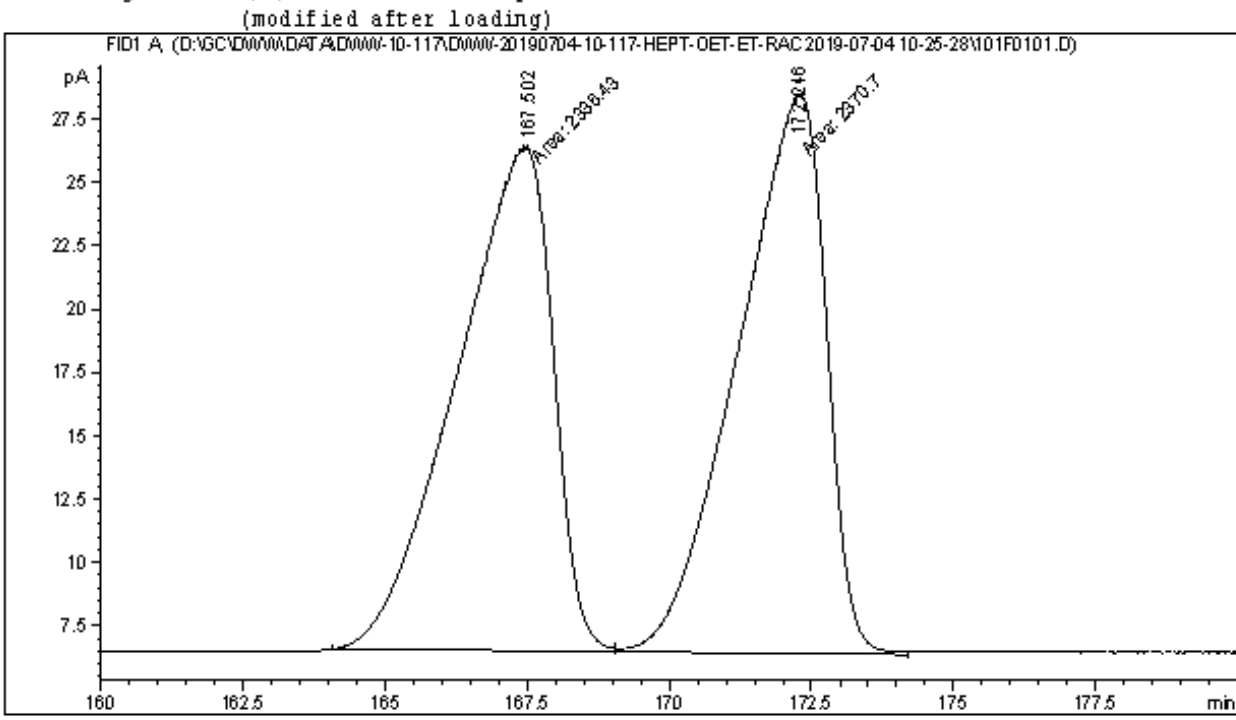

Area Percent Report

\begin{tabular}{|c|c|c|}
\hline Sorted BY & $:$ & Signal \\
\hline Mult iplier & $:$ & 1.0000 \\
\hline Dilution & : & 1.0000 \\
\hline
\end{tabular}

Use Multiplier $\&$ Dilution Factor with ISTDs

Signal 1: FID $1 \mathrm{~A}$

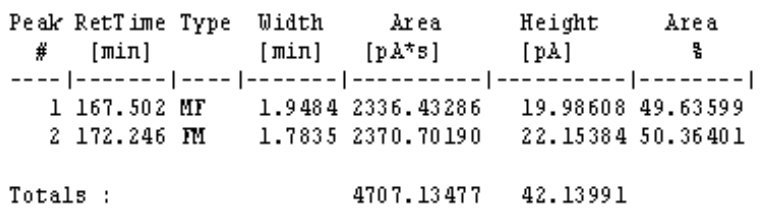

त*त End of Report 


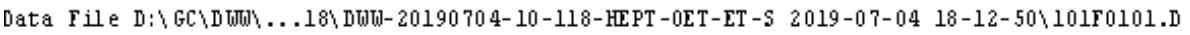
Sample Ilame: Hept-0Et-Et-S

\begin{tabular}{|c|c|c|}
\hline Ac q. Operat or : & : DDTा & Seq. Line : \\
\hline Ac q. Inst rument : & : Inst rument 2 & Location: Vial 101 \\
\hline Injection Date : & : 04-Jul-19, 18:13:53 & $\begin{array}{rlll}\text { Iлј : } & & 1 \\
\text { Irj Volume } & 5 & \text { Hl }\end{array}$ \\
\hline
\end{tabular}

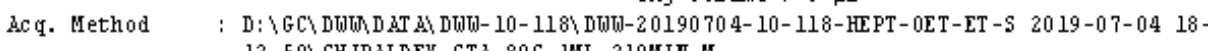
$12-50$ CH IRALDEX -GTA-80C- $1 M L-210 \mathrm{MIN}$. M

Last charged : $7 / 4 / 2019$ 10:20:38 AM by Dit]

Aralys is Method : D : $\backslash$ GC $\backslash$ LYI MET HOD BETA-DEX-390-80C-90M III-140C-30M III- 19ML .M

Last charged : 5/2/2030 6:39:58 M bY L W (modified after loading)
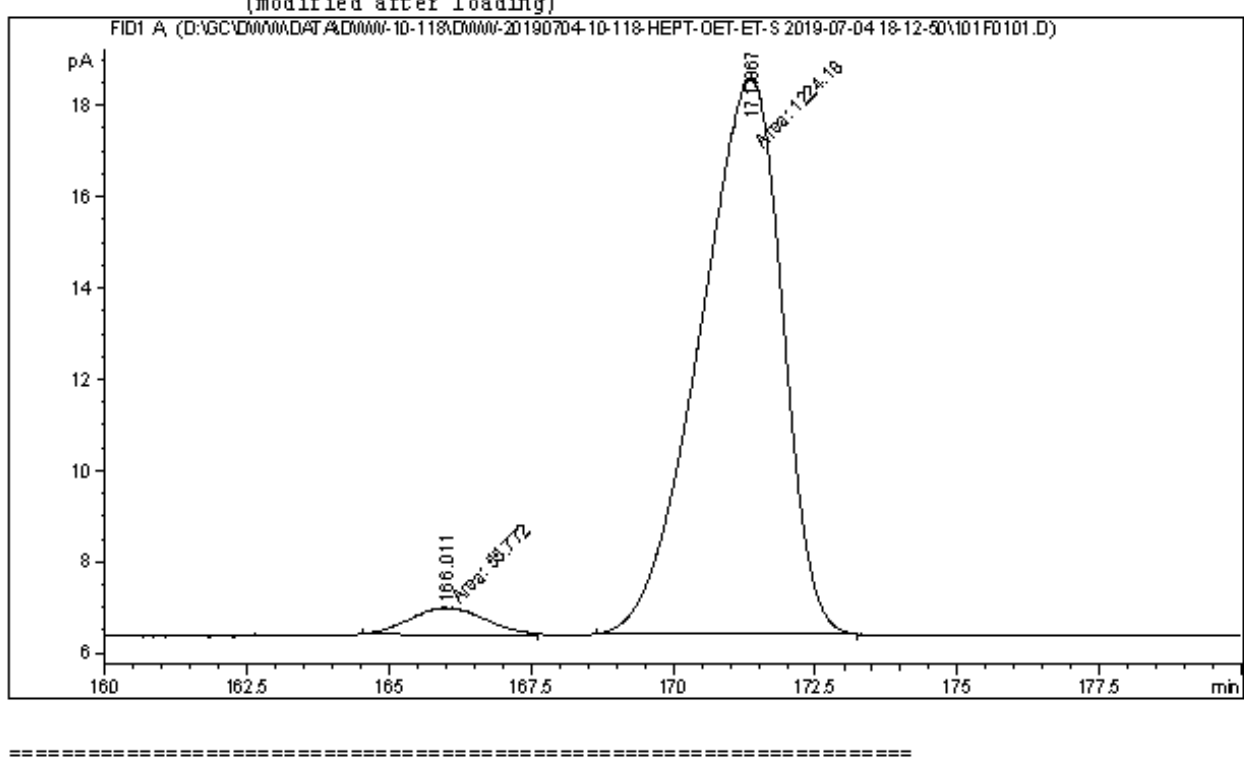

Area Percent Report

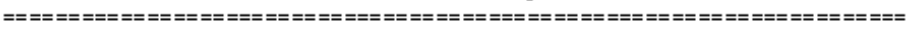

$\begin{array}{lll}\text { Sorted BY } & : & \text { Signal } \\ \text { Multiplier } & : & 1.0000 \\ \text { Dillution } & : & 1.0000\end{array}$

Dilution

1.0000

1.0000

Use Multiplier s Dilution Factor with ISTDs

Signal l: FID 1 ,

\begin{tabular}{|c|c|c|c|c|}
\hline $\begin{array}{l}\text { Peak RetT ime Type } \\
\# \text { [miл] }\end{array}$ & $\begin{array}{l}\text { Width } \\
\text { [min] }\end{array}$ & $\begin{array}{r}\text { hyea } \\
{\left[\mathrm{pH}^{*} \mathrm{~s}\right]}\end{array}$ & $\begin{array}{l}\text { Height } \\
\text { [pa] }\end{array}$ & $\underset{\substack{\text { Area } \\
\text { 믑 }}}{ }$ \\
\hline$--\mid-----1$ & - & ------ & 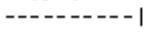 & ---1 \\
\hline $1166.011 \mathrm{MM}$ & 1.5477 & 55.77202 & $6.00595 \mathrm{e}-1$ & 4.35735 \\
\hline $2171.367 \mathrm{MM}$ & 1.6718 & 1224.18018 & 12.20437 & 95.64265 \\
\hline otals: & & 1279.95220 & 12.80496 & \\
\hline
\end{tabular}

蔆 End of Report 
<smiles>CC[C@H](CC(=O)OC)C(C)C</smiles>

$(25 m)$

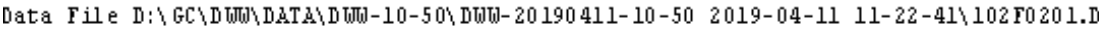
Sample Iame: iP r

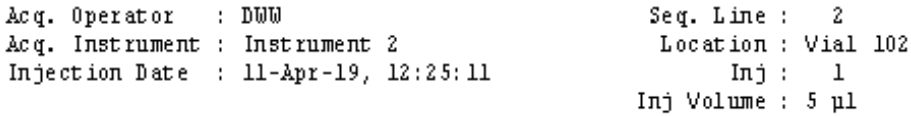

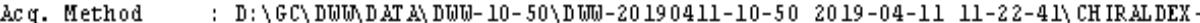
GT $2-60 \mathrm{C}-1 \mathrm{ML}-6 \mathrm{~T}$

Last charged : 4/ 11/2019 10:18:42 M by CC

Aralys is Method : D: \GC LYM MET H0D BETA-DEX-390-80C-90M IIN-140C-30M IN- 19ML .M

Last charged : L//2006 12:14:00 AM bY LYI

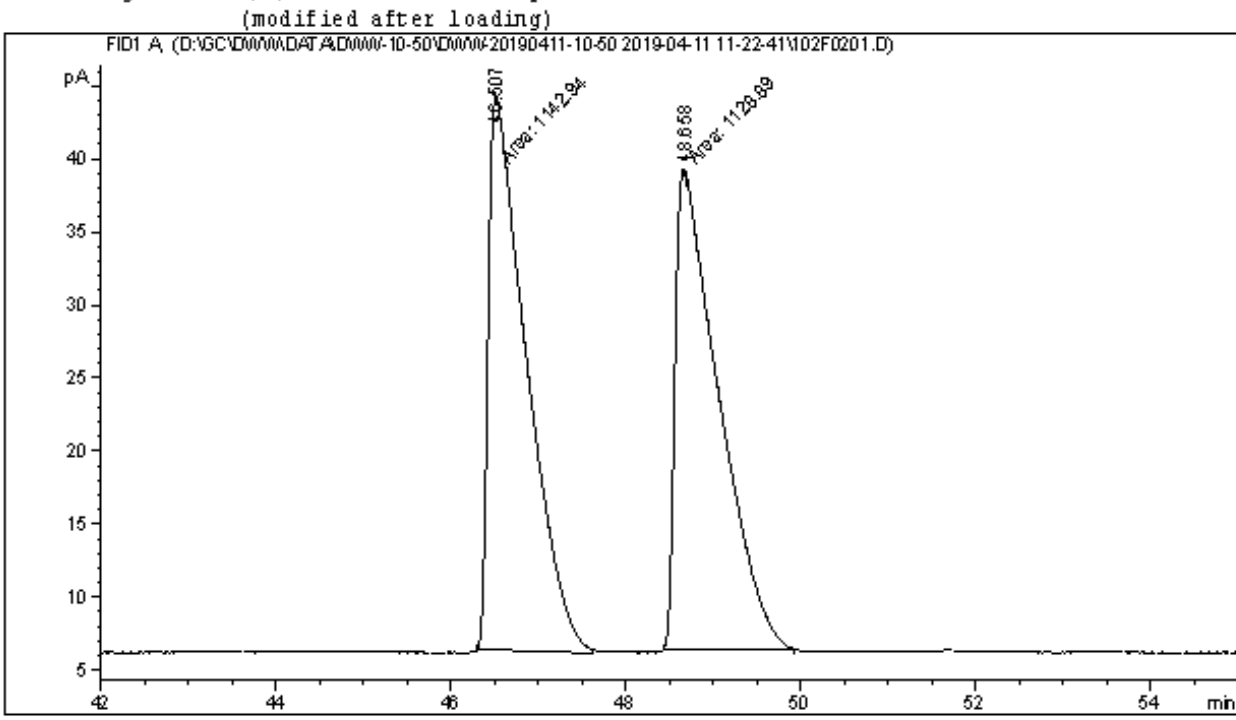

Area Percent Report

\begin{tabular}{|c|c|c|}
\hline Sorted BY & : & Signal \\
\hline Mult iplier & $:$ & 1.0000 \\
\hline Dilution & : & 1.0000 \\
\hline
\end{tabular}

Use Multiplier \& Dilution Factor with ISTDs

Signal 1: FID $1 \mathrm{~A}$

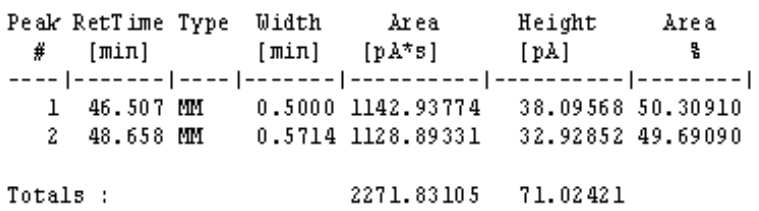

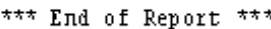




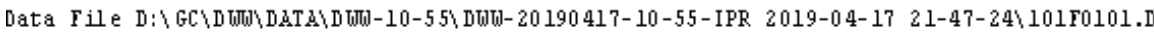
Sample Ilame: Dtw-10-55-iPr

\begin{tabular}{|c|c|c|}
\hline Acq. Operat or : & : Dाדा & Seq. Line: \\
\hline ac q. Inst rument : & : Iлst rumert 2 & Location: Vial 101 \\
\hline Irjection Date & : 17-Apr-19, 21:48:25 & $\begin{array}{rlll}\text { Iлј } & : & & 1 \\
\text { Iлj Volume } & \text { : } & 5 & \text { hl }\end{array}$ \\
\hline
\end{tabular}

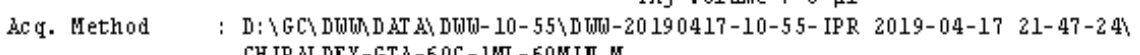
CH IR ALDEX-GTA-60C-1ML-60MIII. M

Last charged: 4/11/2019 10:18:42 3M by CC

Aralys is Method : D: \GC LYM MET HOD BETA-DEX-390-80C-90M IN-140C-30M IN- 19ML .M

Last charged : 5/2/2030 5:32:24 MM bY L W
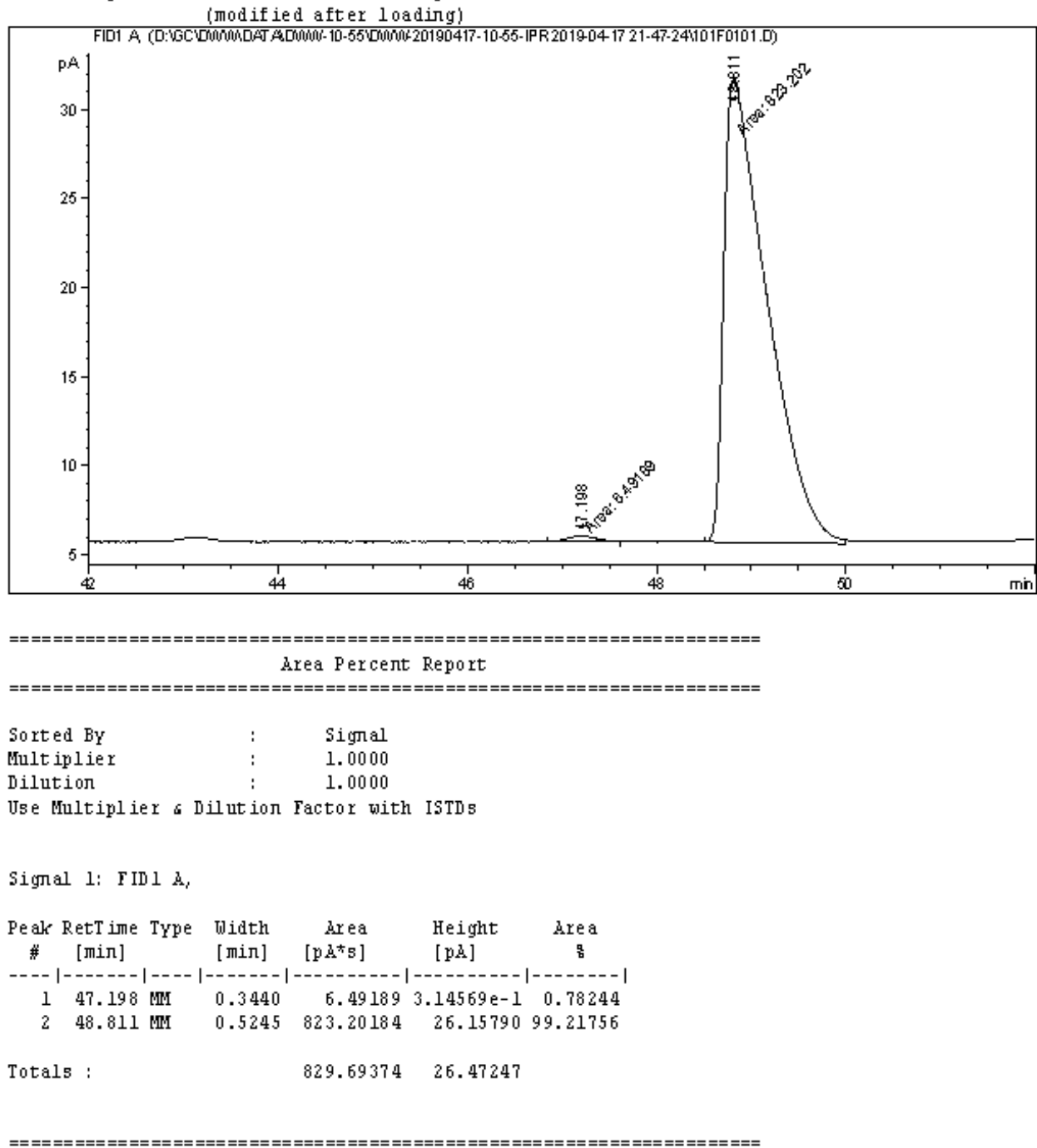

茭 End of Report 
<smiles>CCC(CC(=O)OC)C1CCCCC1</smiles>

$(25 n)$

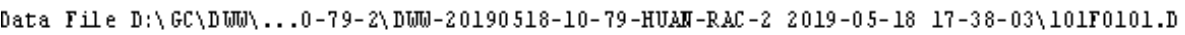
Sample II ame: Dot-10-79-HUAN-RAC-2

\begin{tabular}{|c|c|c|}
\hline Acq. Operator & : DTाד & Seq. Line: \\
\hline dc q. Inst rument & : Inst rument 2 & Location: Vial 101 \\
\hline Injection Date & : 18-MaY-19, 17:39:04 & 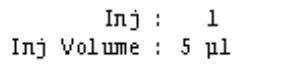 \\
\hline
\end{tabular}

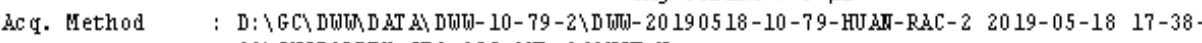
$03 \backslash$ CHIRADEX-GT A-85C-1ML-140MIN . M

Last charged : 5/ 18/2019 5:36:38 PM bY DDTJ

Aralys is Method : D: \GC LYM MET HOD BETA-DEX-390-80C-90M IIN-140C-30M IN- 19ML .M

Last charged : 5/2/2030 6:14:54 WM bY L Y

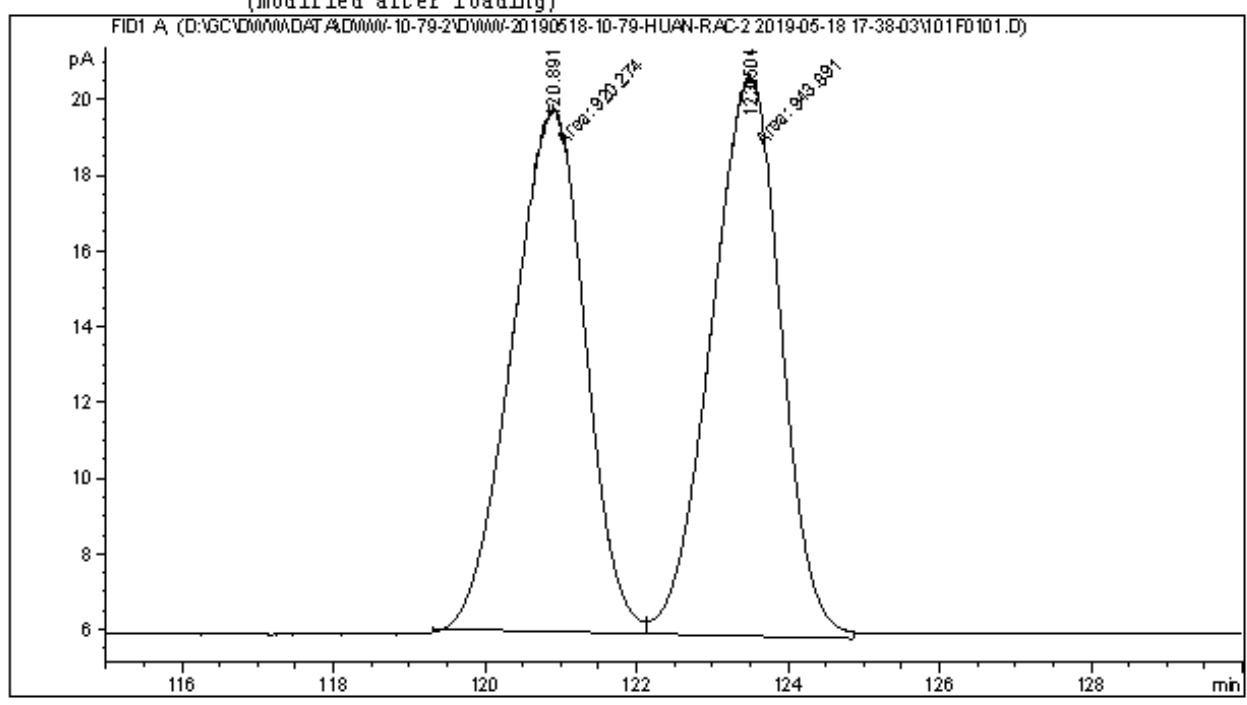

Area Percent Report

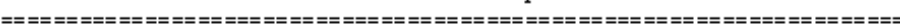

$\begin{array}{lll}\text { Sorted BY } & : & \text { Signal } \\ \text { Multiplier } & : & 1.0000 \\ \text { Dilution } & : & 1.0000\end{array}$

Dilution

Use Multiplier s Dilution Factor with ISTDs

Signal 1: FIDIA,

\begin{tabular}{|c|c|c|c|c|}
\hline $\begin{array}{l}\text { Peak RetT ime TYpe } \\
\# \quad \text { [miл] }\end{array}$ & $\begin{array}{l}\text { Width } \\
{[\text { mir] }}\end{array}$ & $\begin{array}{r}\text { hrea } \\
{\left[\mathrm{ph}^{*} \mathrm{~s}\right]}\end{array}$ & $\begin{array}{l}\text { Height } \\
{[\mathrm{pA}]}\end{array}$ & $\begin{array}{c}\text { Area } \\
\text { 믑 }\end{array}$ \\
\hline$----|------|---$ & ----1 & 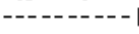 & $-1---1$ & $|-------|$ \\
\hline $1120.891 \mathrm{Mr}$ & 1.1012 & 920.27356 & 13.92879 & 49.36655 \\
\hline $2123.504 \mathrm{M}$ & 1.0601 & 943.89050 & 14.83900 & 50.63345 \\
\hline Totals : & & 1864.16406 & 28.76779 & \\
\hline
\end{tabular}

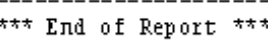




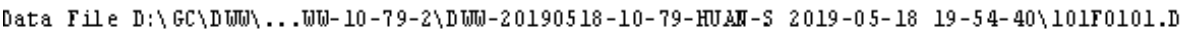
Sample IIame: DDth-10-79-HULI-S

\begin{tabular}{|c|c|c|}
\hline Acq. Operator & : DWW & Seq. L іле : \\
\hline Ac q. Inst rument & : Inst rument 2 & Location: Vial 101 \\
\hline Irjection Date & : 18-MaY-19, 19:55:39 & \begin{tabular}{rlc} 
Iлj : & \multicolumn{1}{c}{} \\
Iлj Volume & 5 l
\end{tabular} \\
\hline
\end{tabular}

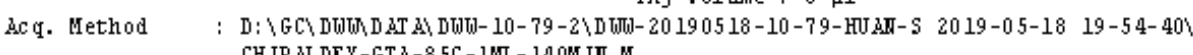
CH IR AL DEX - GTA-8 5C-1ML- $140 \mathrm{MIN}$.M

Last charged : 5/18/2019 5:36:38 PU by Diti

Aralys is Method : D : \GC LYI MET HOD BETA-DEX-390-80C-90M III-140C-30M IN- 19ML .M

Last charged : 5/2/2030 6:17:33 M bY L W (modified after loading)
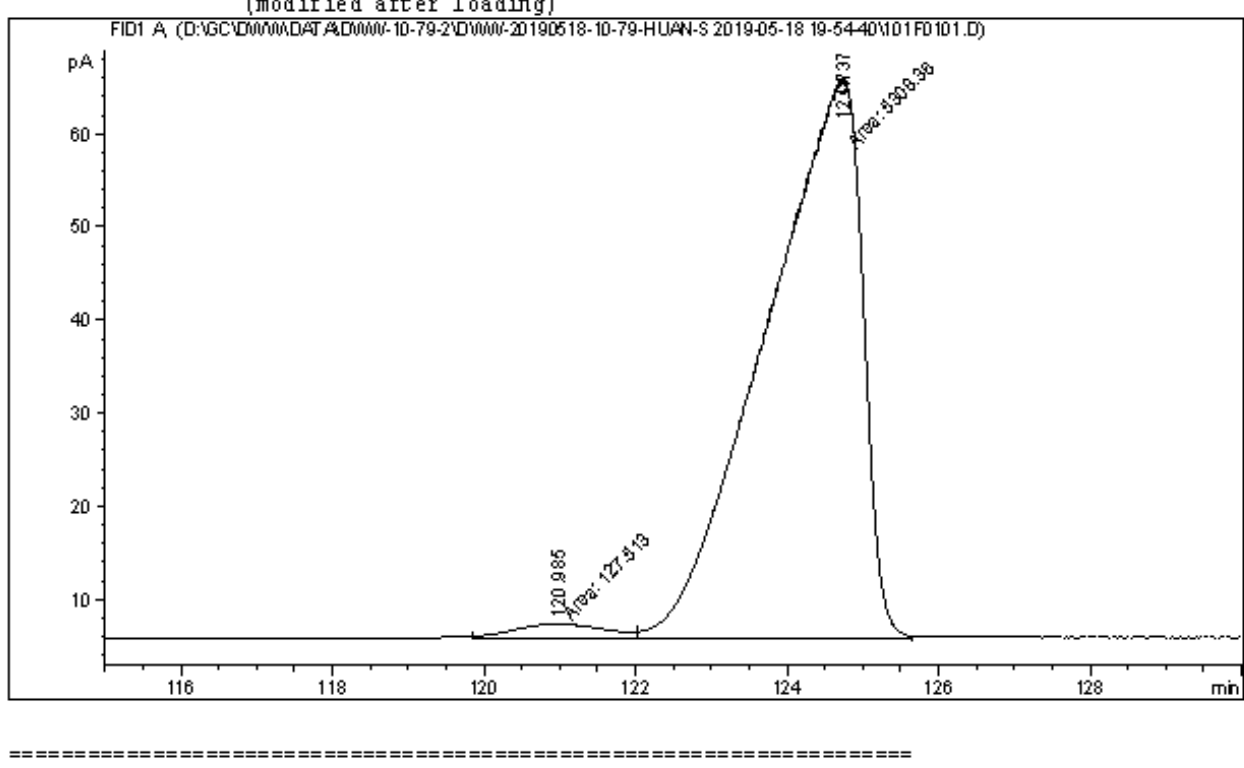

Area Percent Report

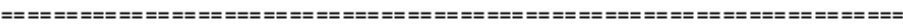

$\begin{array}{lll}\text { Sorted By } & : & \text { Signal } \\ \text { Multiplier } & : & 1.0000\end{array}$

Dilution

1.0000

Dilution

1.0000

Use Multiplier s Dilution Factor with ISTDs

Signal l: FID 1 ,

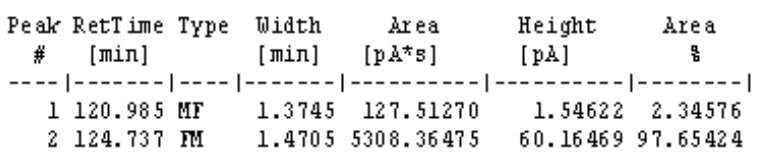

Totals : $\quad 5435.87744 \quad 61.71091$

त药 Erd of Report 
<smiles>CCC(CC(=O)OC)c1ccccc1</smiles>

(250)

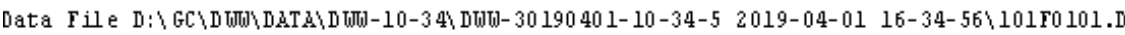
Sample IIame: Dtw-10-34
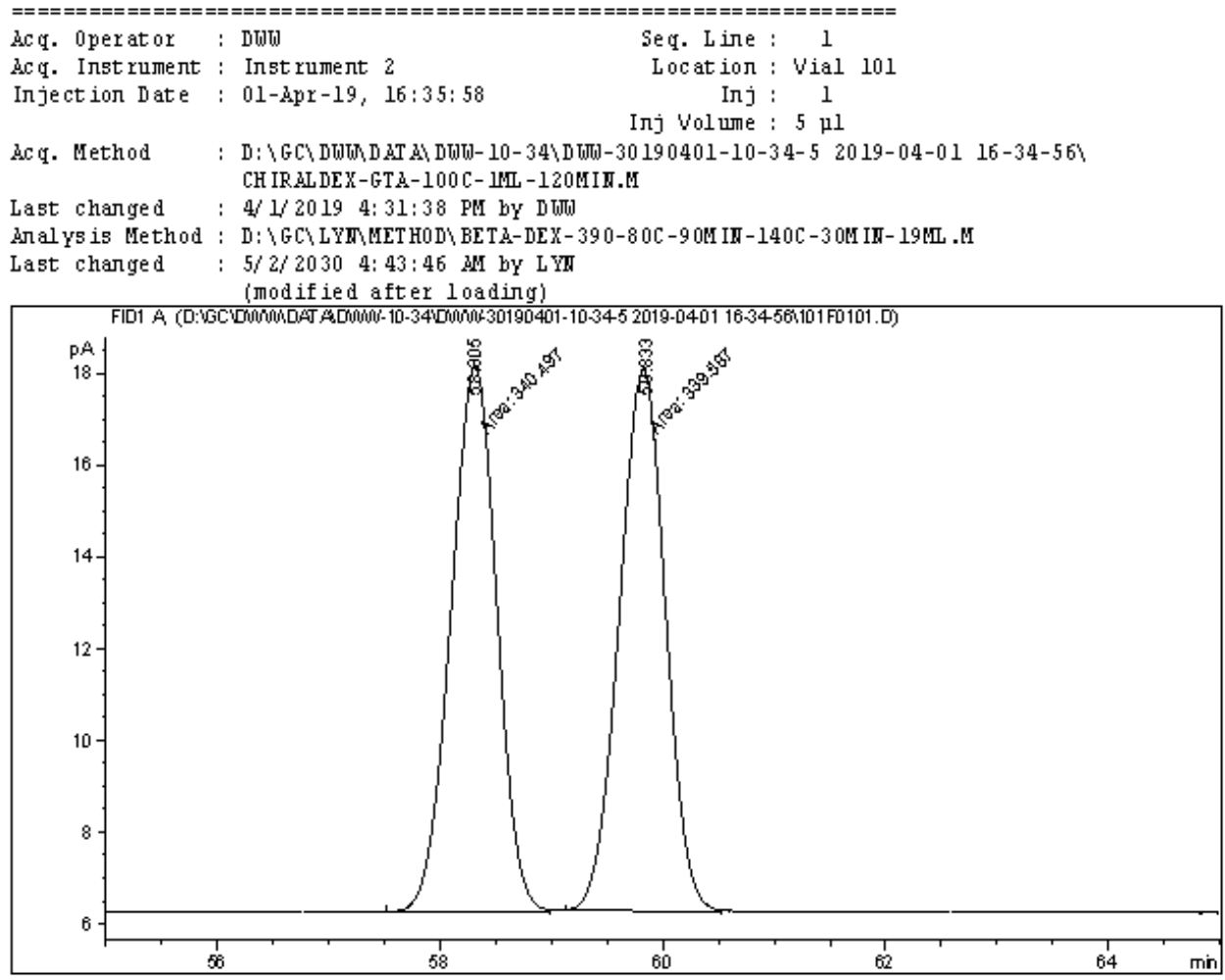

Area Percent Report

$\begin{array}{lll}\text { Sorted BY } & : & \text { Signal } \\ \text { Multiplier } & : & 1.0000 \\ \text { Dilution } & : & 1.0000\end{array}$

Dilution

Use Multiplier s Dilution Factor with ISTDs

Signal l: FIDI A,

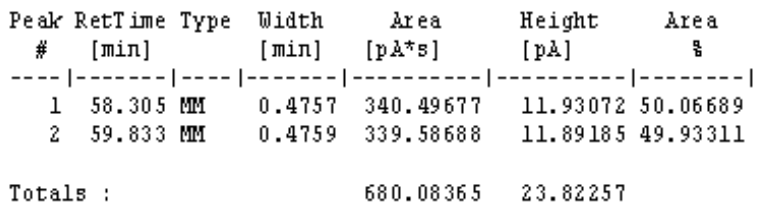

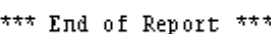




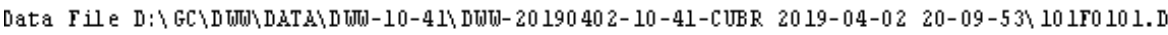
Sample IIame: CuBrSMe2

\begin{tabular}{|c|c|c|}
\hline Acq. Operat or : & : Dाדा & Seq. Line: \\
\hline ac q. Inst rument : & : Iлst rumert 2 & Location: Vial 101 \\
\hline Irjection Date & : 02-Apr-19, 20:10:55 & $\begin{array}{rlll}\text { Iлј } & : & & 1 \\
\text { Iлj Volume } & \text { : } & 5 & \text { hl }\end{array}$ \\
\hline
\end{tabular}

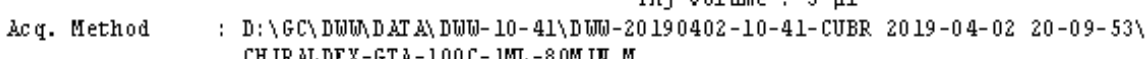
CH IR ALDEX-GTA-100C- $1 \mathrm{ML}-80 \mathrm{~N}$ II .M

Last charged : 4/2/2019 8:09:52 PM by DJT

Aralys is Method : D: \GC LYM MET HOD BETA-DEX-390-80C-90M IIN-140C-30M IN- 19ML .M

Last charged : 5/2/2030 4:48:50 MM bY LYM
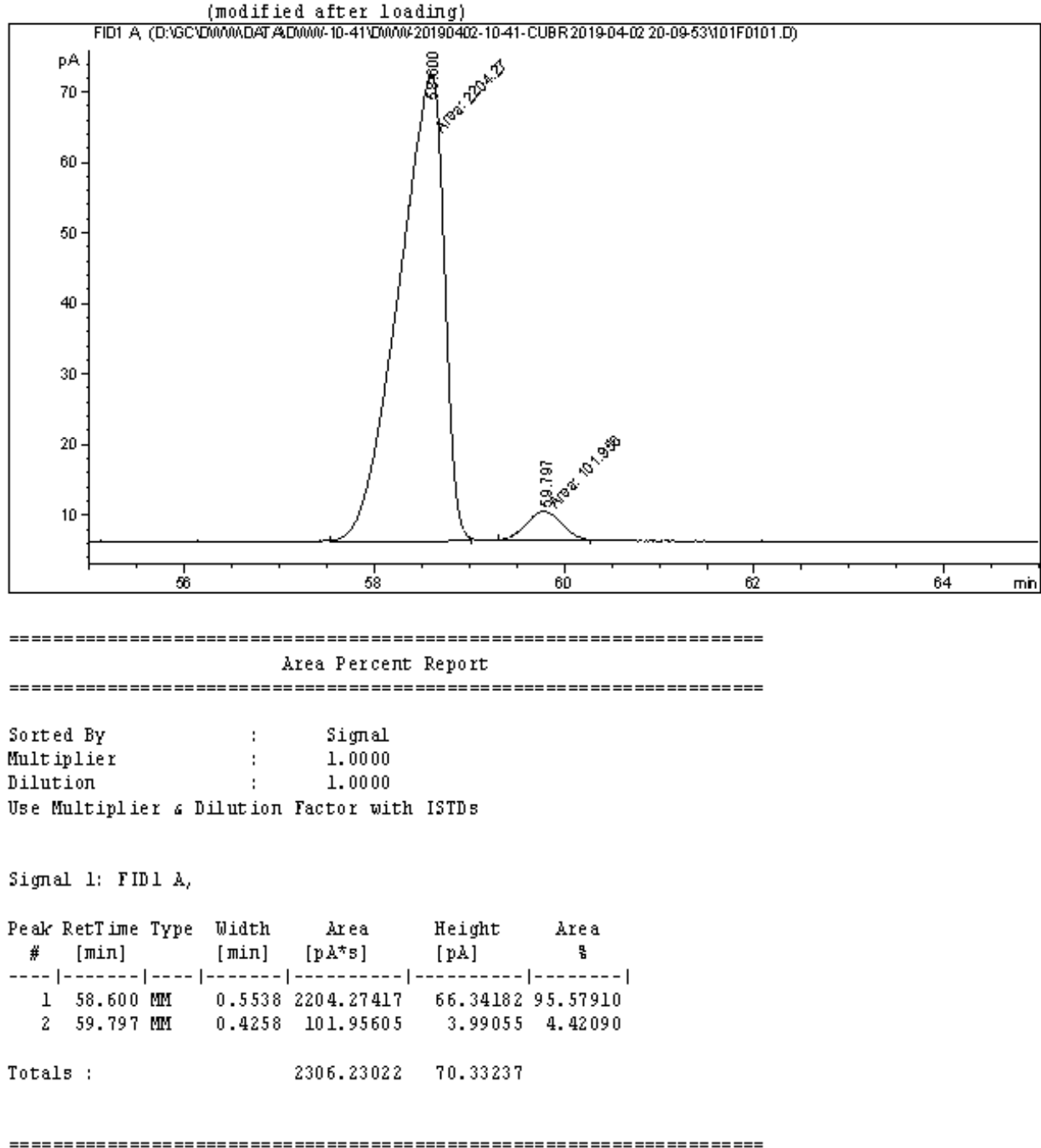

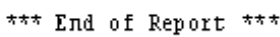


<smiles>CC[C@H](CC(=O)OC)Cc1ccccc1</smiles>

(25p)

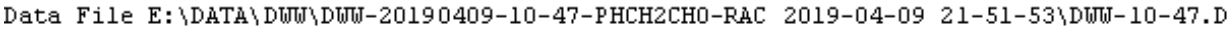
Sample Name: PhCH2CHO

\begin{tabular}{|c|c|c|c|}
\hline Ácq. Operator & : SYSTEM & Seq. Line : & 1 \\
\hline Ac q. Instrument & : 1260 & Location : & 5 \\
\hline Injection Date & : 4/9/2019 9:53:22 PM & Inj : & 1 \\
\hline
\end{tabular}

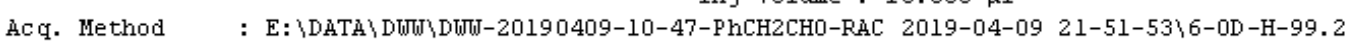
$-0.8-220 \mathrm{MM}-1 \mathrm{ML}-10 \mathrm{ul}-20 \mathrm{~min}-\mathrm{ALL}$. M

Last changed : 4/9/2019 9:51:54 PM by SYSTEM

Analysis Method : E: \DATA \DाJ\D相-20190409-10-47-PhCH2CH0-RAC 2019-04-09 21-51-53\6-0D-H-99.2 -0.8-220MM-1ML-10ul-20min-ALL.M (Sequence Me thod)

Last changed : 7/24/2020 12:42:37 PM by SYSTEM (modified after loading)

Additional Info : Peak (s) manually integrated

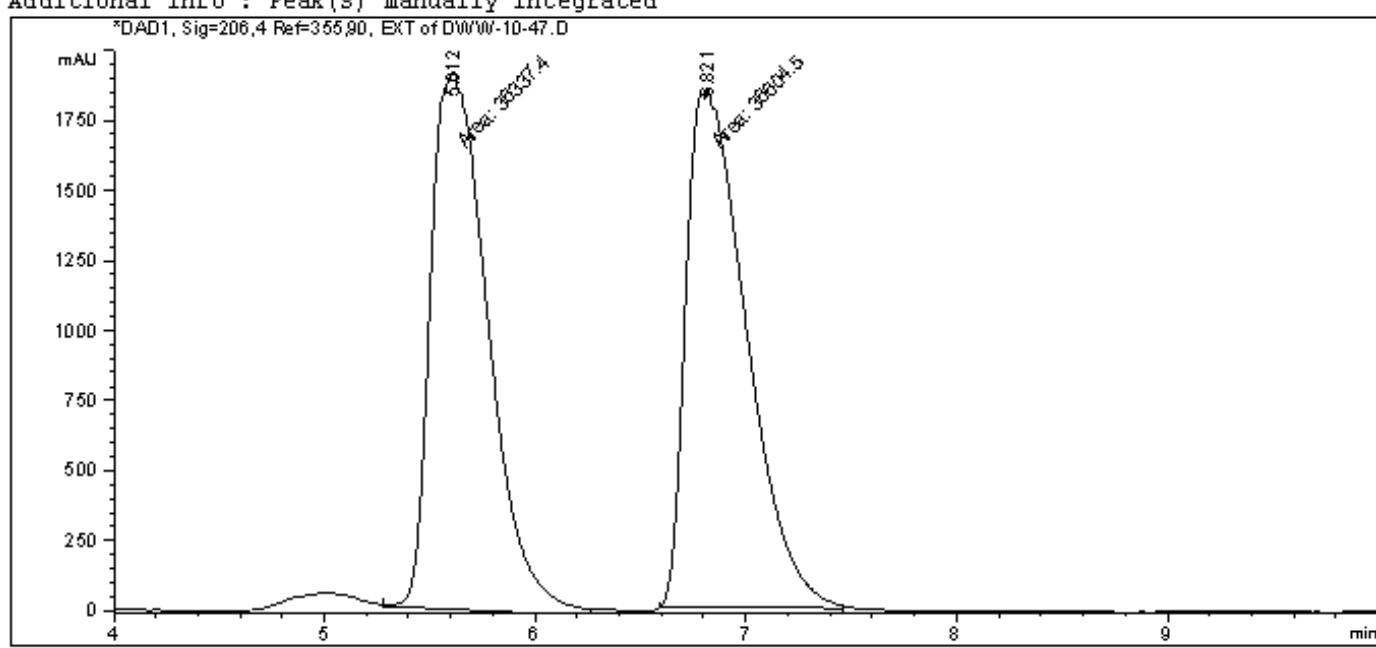

Area Percent Report

\begin{tabular}{|c|c|}
\hline Sorted BY & Signal \\
\hline Multiplier & 1.0000 \\
\hline Dilution & $: \quad 1.0000$ \\
\hline
\end{tabular}

Do not use Multiplier \& Dilution Factor with IsTDs

Signal 1: DAD1, Sig=206, 4 Ref $=355,90$, EXT

signal has been modified after loading from rawdata file!

\begin{tabular}{|c|c|c|c|c|c|c|}
\hline $\begin{array}{c}\text { Peak } \\
\#\end{array}$ & $\begin{array}{c}\text { RetTime } \\
\text { [min] }\end{array}$ & Type & $\begin{array}{l}\text { Width } \\
\text { [min] }\end{array}$ & $\begin{array}{c}\text { Area } \\
\text { [mAUts }]\end{array}$ & $\begin{array}{l}\text { Height } \\
\text { [mAU] }\end{array}$ & $\begin{array}{c}\text { Area } \\
\stackrel{5}{*}\end{array}$ \\
\hline & & & & & & \\
\hline & 5 & & 0.3 & 3.63 & 1923 & 49 \\
\hline & 6. & & 306 & 3.66 & 1845.56592 & 183. \\
\hline
\end{tabular}

Totals :

$7.29419 \mathrm{e} 4 \quad 3768.69946$ 


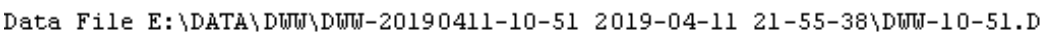
Sample Name: DotJ-10-51
Acq. Operator : SYSTEM
Acq. Instrument : 1260
Seq. Line : 1
Injection Date : 4/11/2019 9:57:09 PM
Location : 3
Inj : 1
Inj Volume : $10.000 \mu l$

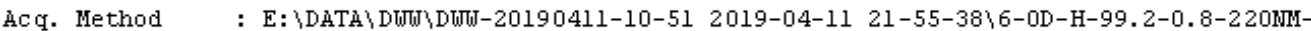
1ML-10ul-20min-ALL.M

Last changed : 4/11/2019 10:08:08 PM by SYSTEM

(modified after loading)

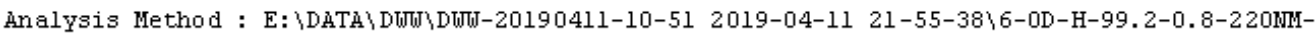
1ML-10ul-20min-ALL.M (Sequence Method)

Last changed : 7/23/2020 11:45:41 PM by SYSTEM

(modified after loading)

Additional Info : Peak ( $s$ ) manually integrated

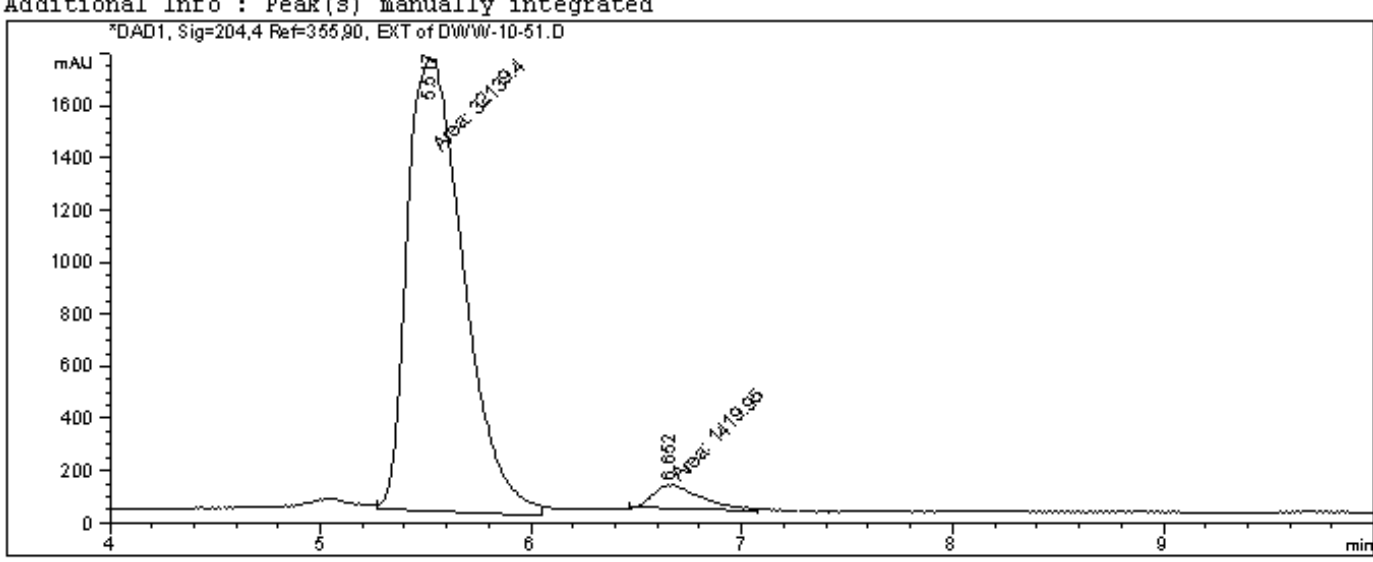

Area Percent Report

$\begin{array}{lll}\text { Sorted By } & : & \text { signal } \\ \text { Multiplier } & : & 1.0000 \\ \text { Dilution } & : & 1.0000\end{array}$

Do not use Multiplier \& Dilution Factor with IsTDs

Signal 1: DADl, Sig=204, 4 Ref $=355,90$, EXT

signal has been modified after loading from rawdata file!

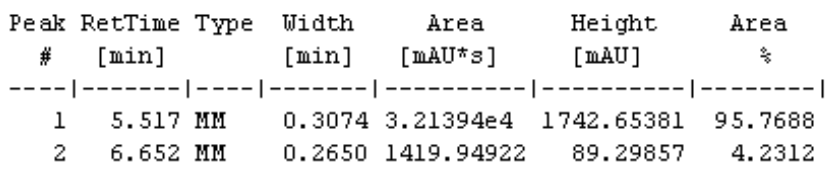

Totals : $\quad 3.35593 \mathrm{e} 4 \quad 1831.95238$

ネネー End of Report *ネ* 
<smiles>CC[C@@H](CC(=O)OC)c1ccco1</smiles>

(25q)

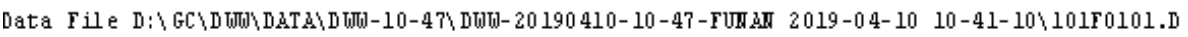
Sample IIame: Dow-10-47-FUस
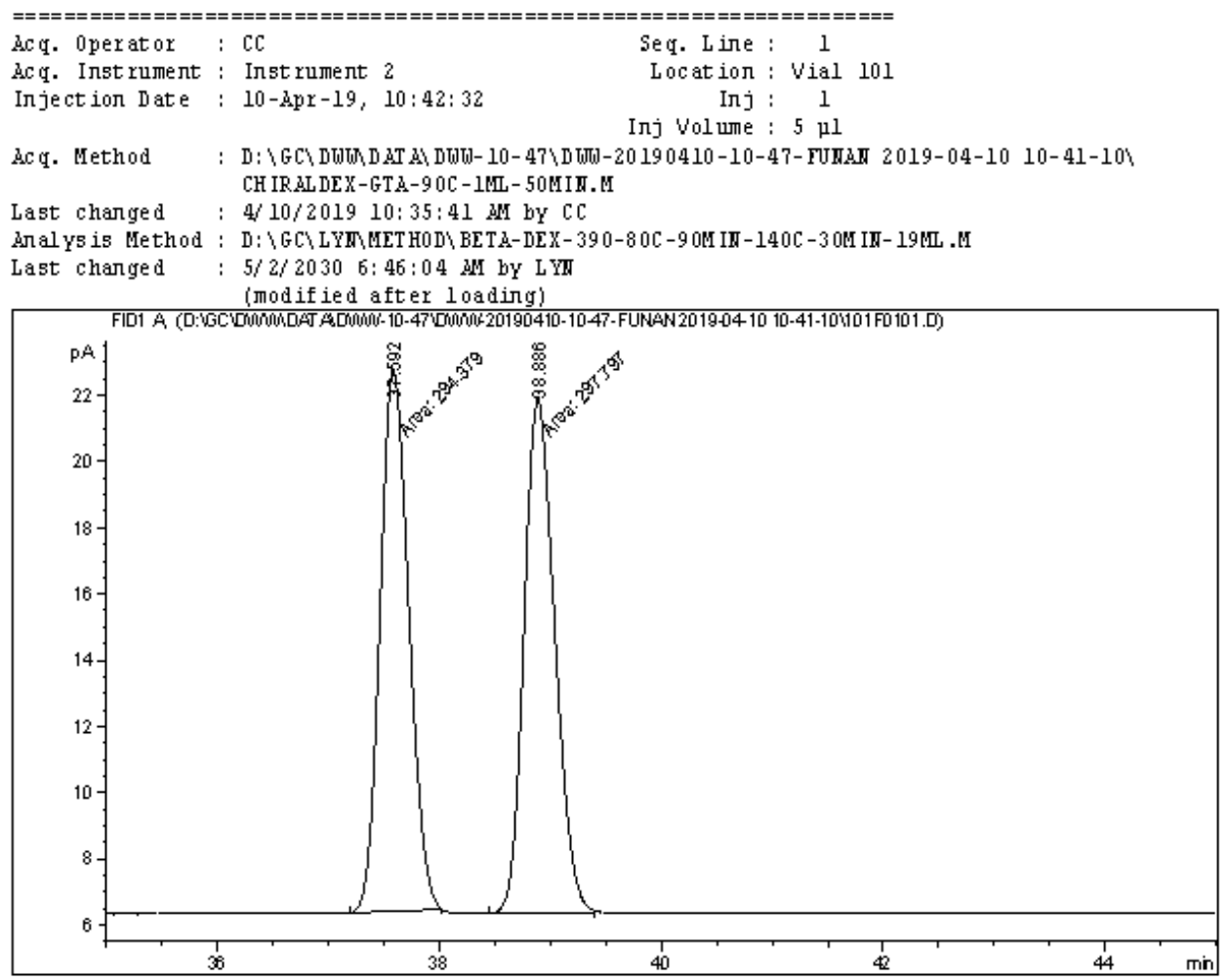

Area Percent Report

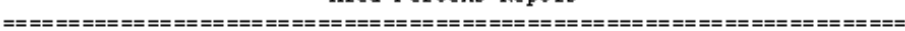

$\begin{array}{lll}\text { Sorted BY } & : & \text { Signal } \\ \text { Multiplier } & : & 1.0000 \\ \text { Dilution } & : & 1.0000\end{array}$

Use Multiplier s Dilution Factor with ISTDs

Signal l: FID 1 ,

\begin{tabular}{|c|c|c|c|c|c|}
\hline $\begin{array}{c}\text { Peak } \\
\#\end{array}$ & $\begin{array}{l}\text { RetT ime TYpe } \\
\text { [miл] }\end{array}$ & $\begin{array}{l}\text { Width } \\
\text { [mis] }\end{array}$ & $\begin{array}{r}\text { Area } \\
\left.\text { [p } \mathrm{p}^{*} \mathrm{~s}\right]\end{array}$ & $\begin{array}{l}\text { Height } \\
\text { [pA] }\end{array}$ & $\begin{array}{c}\text { Area } \\
\text { 믑 }\end{array}$ \\
\hline & $37592 \mathrm{Mr}$ & C. 3989 & 29837898 & 16 & 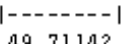 \\
\hline $\begin{array}{l}1 \\
2\end{array}$ & $38.886 \mathrm{MM}$ & 0.3183 & $\begin{array}{r}294.31094 \\
297.79675\end{array}$ & $\begin{array}{l}10.41019 \\
15.59447\end{array}$ & $\begin{array}{l}49.71142 \\
50.28858\end{array}$ \\
\hline [otal & & & 592.17569 & 32.01066 & \\
\hline
\end{tabular}




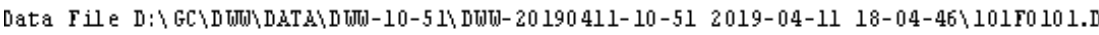
Sample Iame: B-furar

\begin{tabular}{|c|c|c|}
\hline Acq. operat or : & : Dाד & Seq. Line: \\
\hline Ac q. Inst rument : & : Iлst rumert 2 & Location: Vial 101 \\
\hline Iлjection Date : & : 11-Apr-19, 18:05:48 & $\begin{array}{rlll}\text { Iлј } & : & & 1 \\
\text { Iлj Volume } & \text { : } & 5 & \text { hl }\end{array}$ \\
\hline
\end{tabular}

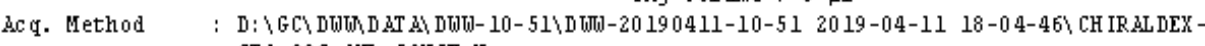
GT A- 90 C- $1 M L-5$ OM IN .M

Last charged : 4/11/2019 6:04:45 PM by Diti

aralys is Method : D : \GC LYI MET HOD BETA-DEX-390-80C-90M III-140C-30M III-19ML .M

Last charged : 5/2/2030 6:48:21 M bY L W

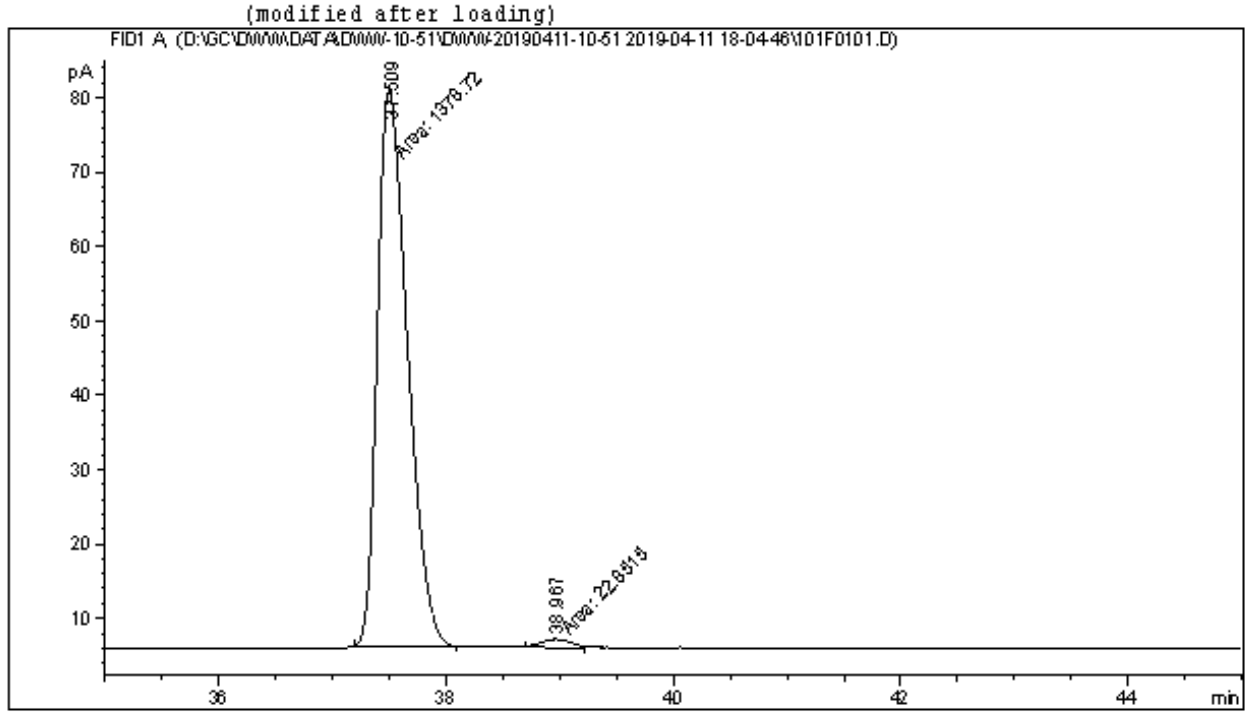

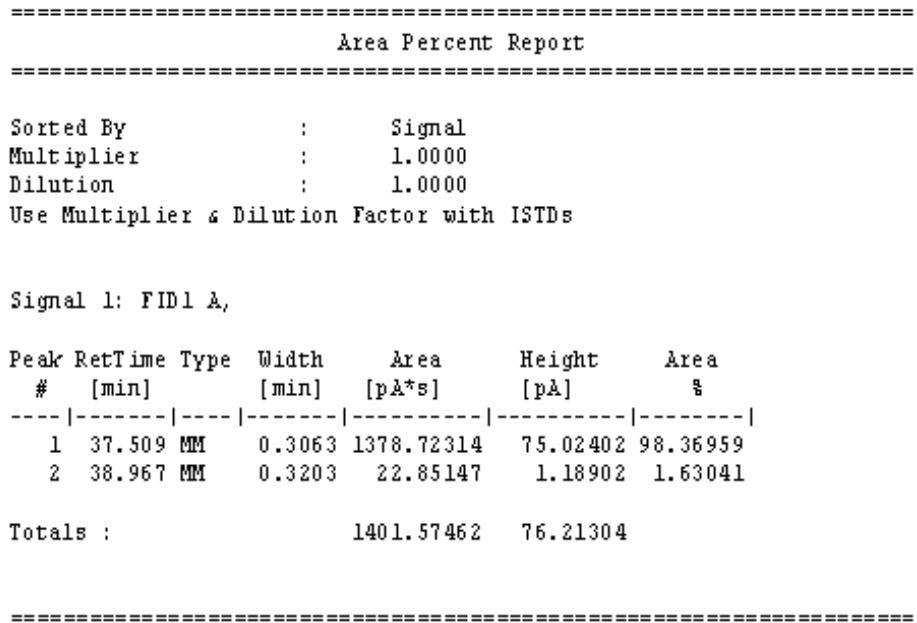

蓅 End of Report 
<smiles>CCC(CC(=O)OC)c1cccs1</smiles>

(25r)

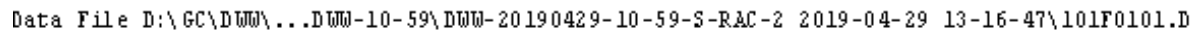
Sample IIame: Doth-10-59-S-rac-2

\begin{tabular}{|c|c|c|}
\hline Acq. Operat or & : DWד & Seq. Liлe : \\
\hline q. Inst rumert & : Inst rumert 2 & Location: Vial 101 \\
\hline Injection Date & : 29-Apr-19, 13:17:49 & $\begin{array}{rlc}\text { Iлj : } & & 1 \\
\text { Iлj Volume : } & 5 \text { pl }\end{array}$ \\
\hline
\end{tabular}

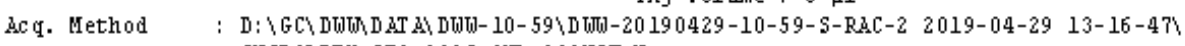
CH IRAL DEX-GTA-100C- 1 LL - 120 MIN.M

Last charged : 4/1/2019 4:31:38 PM bY DIJT

Aлalys is Method : D: \GC LYT MET HOD BETA-DEX-390-80C-90M IN-140C-30M IN- 19ML .M

Last changed : 5/2/2030 $5: 38: 38$ M bY L W

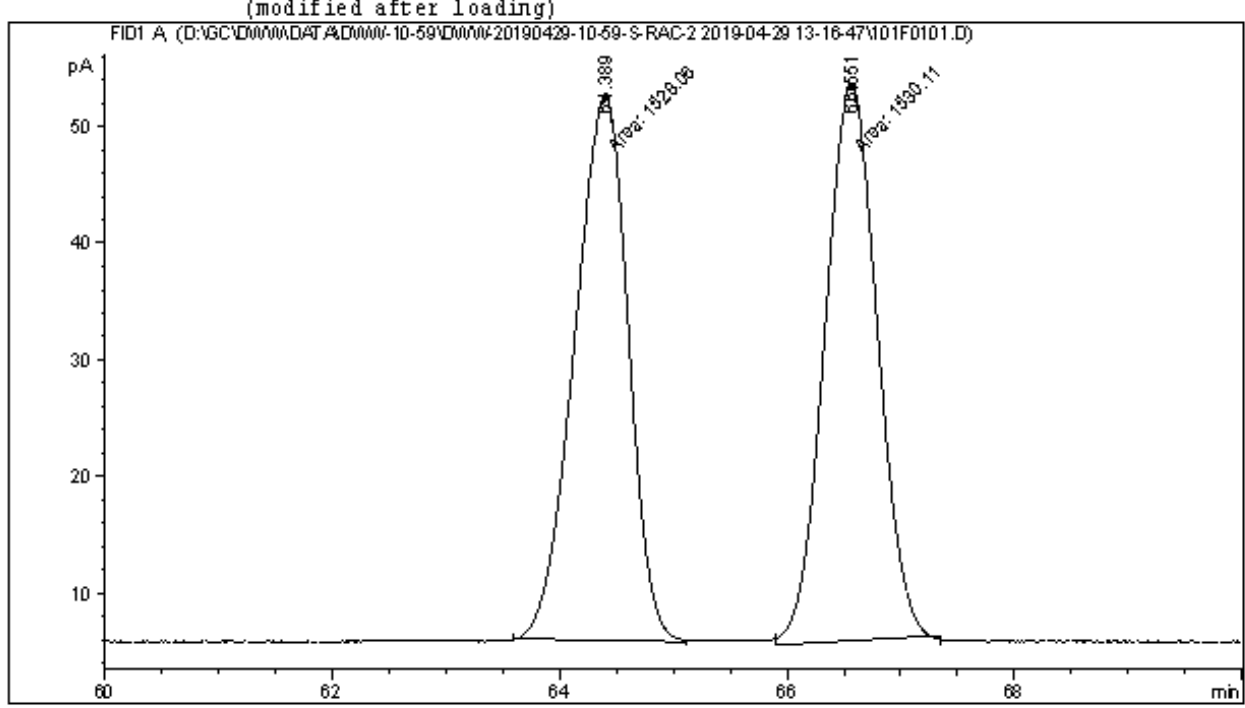

Area Percent Report

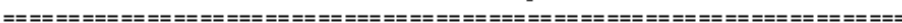

$\begin{array}{lll}\text { Sorted BY } & : & \text { Signal } \\ \text { Multiplier } & : & 1.0000\end{array}$

(2000)

Use Multiplier s Dilution Factor with ISTDs

Signal l: FID $1 \mathrm{~A}$,

\begin{tabular}{|c|c|c|c|c|c|}
\hline $\begin{array}{c}\text { Peak } \\
\#\end{array}$ & $\begin{array}{l}\text { RetT ime TYpe } \\
\text { [mіл] }\end{array}$ & $\begin{array}{l}\text { tidth } \\
\text { [mig] }\end{array}$ & $\begin{array}{r}\text { Area } \\
{\left[\mathrm{pA}^{*} \mathrm{~S}\right]}\end{array}$ & $\begin{array}{l}\text { Height } \\
\text { [ps] }\end{array}$ & $\begin{array}{c}\text { Area } \\
\text { 믑 }\end{array}$ \\
\hline---1 & $|------|-$ & & |-------- & & ----1 \\
\hline 1 & $64.389 \mathrm{MM}$ & 0.5422 & 1528.06335 & 46.96836 & 49.96657 \\
\hline 2 & $66.551 \mathrm{MM}$ & 0.5325 & 1530.10828 & 47.88820 & 50.03343 \\
\hline Total & : & & 3058.17163 & 94.85655 & \\
\hline
\end{tabular}

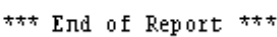




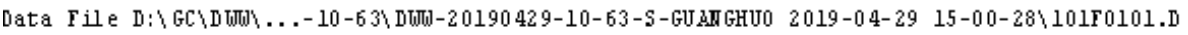
Sample Ilame: Dtw-10-63-S-guarghuo

\begin{tabular}{|c|c|c|}
\hline Acq. Operator & : DWW & Seq. Liлe : \\
\hline Ac q. Inst rument & : Inst rument 2 & Location: Vial 101 \\
\hline Irjection Date & : 29-Apr-19, $15: 01: 31$ & $\begin{array}{rlll}\text { Iлj : } & & 1 \\
\text { Irj Volume } & 5 & \text { pl }\end{array}$ \\
\hline
\end{tabular}

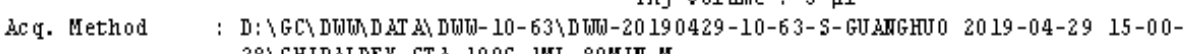
$28 \backslash$ CHIRALDEX - GT A- $100 \mathrm{C}-1 \mathrm{ML}-80 \mathrm{MIII}$.

Last charged : 4/2/2019 8:09:52 PM by DJTा

Aralys is Method : D: \GC LYM MET HOD BETA-DEX-390-80C-90M IN-140C-30M IN- 19ML .M

Last changed : 5/2/2030 5:43:44 3 bY LYM (modified after loading)

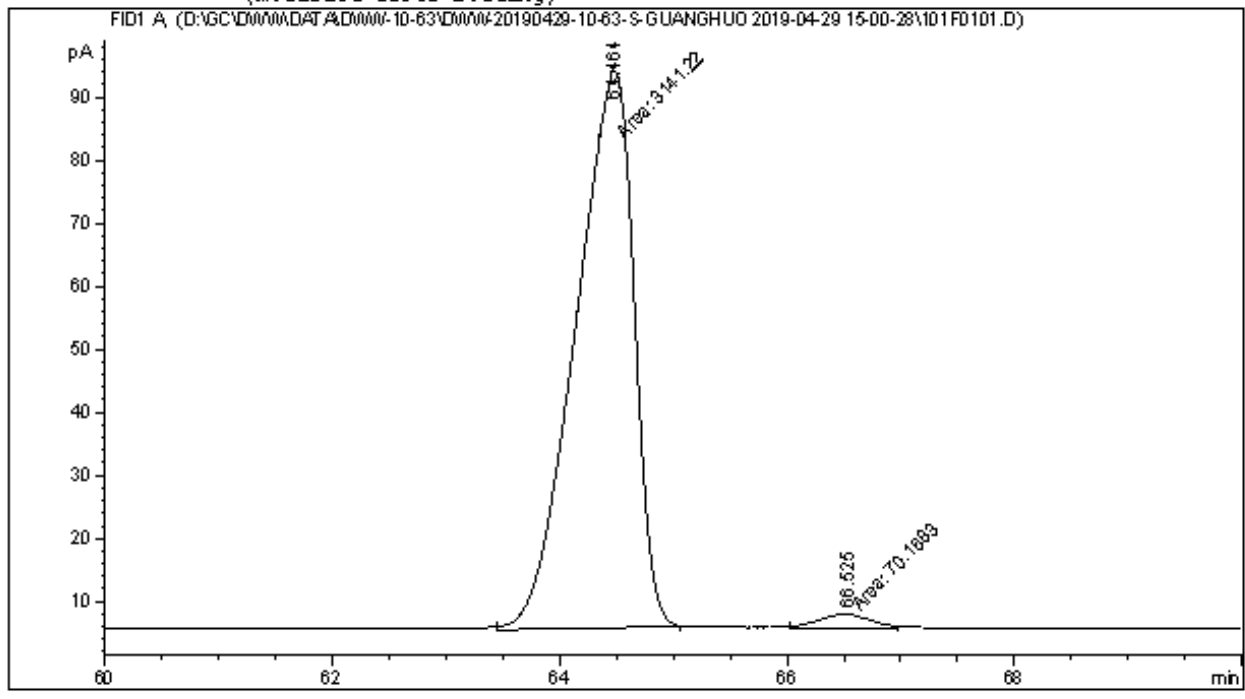

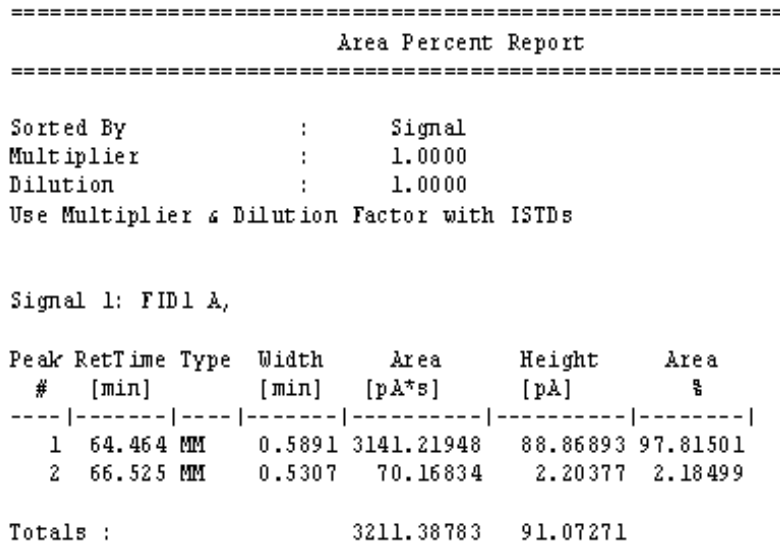

药 End of Report 